\title{
Gorgonian Responses to Environmental Change on Coral Reefs in SE Sulawesi, Indonesia
}

\author{
Sonia J. Rowley
}

\author{
A thesis submitted to \\ Victoria University of Wellington \\ in fulfilment of the requirements for the degree of \\ Doctor of Philosophy in Science
}

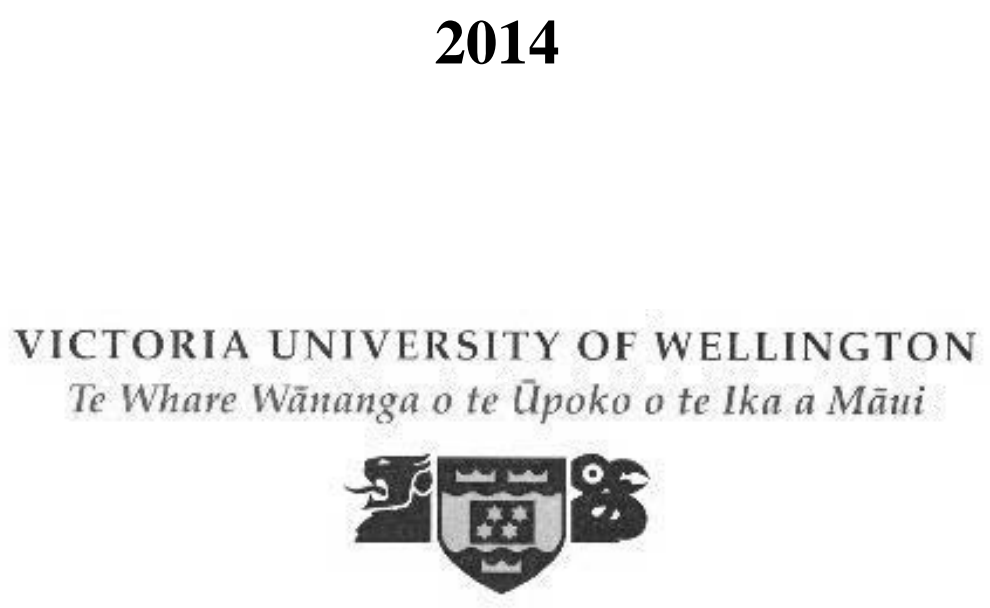


Dedicated entirely to my family, Mike, Penny and Giles Rowley, whose love knows no bounds. 


\begin{abstract}
Gorgonian corals (Cnidaria: Anthozoa: Octocorallia) are conspicuous, diverse and often dominant components of benthic marine environments. Intra- \& interspecific morphological variability in gorgonians are influenced by environmental factors such as light, sedimentation and flow rates. Yet, little is known about the responses of gorgonian taxa to environmental parameters particularly in Indonesia, despite their high regional abundance and diversity. With a burgeoning human population and subsequent marine resource exploitation, reefs throughout the Indonesian archipelago are under rapid decline and often destroyed. Conservation surveys are however, underway with a tendency to overlook gorgonian taxa primarily due to unresolved taxonomic assignment leading to difficulties in field identification.
\end{abstract}

The aims of this study were to: 1) characterise gorgonian diversity and ecology across a gradient of habitat quality within the Wakatobi Marine National Park (WMNP), SE Sulawesi, Indonesia, 2) assess morphological and genetic variability between morphotypes of the ubiquitous zooxanthellate isidid Isis hippuris Linnaeus 1758 from healthy and degraded reefs, 3) determine if I. hippuris morphotypes are environmentally induced (plastic) or genetically derived through reciprocal transplant experiments (RTEs) between contrasting reefs and thus, 4) identify mechanisms of plasticity capacity or divergence through phenotypic trait integration in response to environmental change.

Ecological surveys revealed considerable gorgonian diversity with a total of 197 species and morphotypes from 42 genera, and 12 families within the suborders Calcaxonia and Holaxonia and the group Scleraxonia, with current estimates of over 21 new species and 28 new species records for the region. Gorgonian abundance and diversity increased with reef health and bathymetry. However, a clear loss of gorgonian diversity existed with increased sedimentation and reduced light due to anthropogenic disturbance. In particular, two distinct I. hippuris morphotypes were highly abundant between environmental clines: short-branched multi/planar colonies on healthy reefs, and long-branched bushy colonies on degraded reefs. Comparative morphological and molecular analyses using ITS2 sequence and predicted secondary structure, further corroborated haplotype differences relative to morphotypes between environments. However, unsatisfactory assignment of I. hippuris morphotypes to previously described alternatives (Isis reticulata Nutting 1910, Isis minorbrachyblasta Zou, Huang \& Wang 1991) questions the validity to such taxonomic assignments. Phylogenetic analyses also confirm that 
the polyphyletic nature of the Isididae lies in its type species I. hippuris, being unrelated to the rest of its family members.

A one-year RTE revealed three key results, that: 1) reduced survivorship of healthy reef morphotypes on degraded reefs implied the onset of lineage segregation through immigrant inviability, 2) prominent phenotypic traits were at the morphological and bio-optical levels revealing high phenotypic plasticity in healthy clones, and relative insensitivity to environmental change in degraded reef morphotypes, indicative of local adaptation leading to incipient ecological divergence, and 3) photoacclimation at the bio-optical level was not attributed to endosymbiont diversity or shuffling, with all test colonies possessing a novel clade D1a Symbiodinium.

While it is clear that gorgonian taxa within the WMNP are of exceptional diversity and abundance, responses to environmental perturbation highlight three pertinent, testable ideas. Firstly, increased species richness specifically with depth in azooxanthellate taxa, invite tests of deep-reef refugia previously established through geological change. Secondly, ecological assessment targets research on informative taxa for focused systematics and mechanisms of phenotypic divergence. Thirdly, exploring intrinsic and extrinsic interactions that define the host-symbiont relationship and differential biological success using physiological and next generation sequencing approaches. These objectives would provide considerable insight into the evolutionary processes to environmental change, accelerated by anthropogenic encroachment.

Taken together, this work signifies that gorgonian corals within the WMNP are of foremost diversity and concern, exhibiting informative ecological and mechanistic responses to environmental perturbation. This evidence elicits tests of deep-reef refugia, priority systematics, mechanisms of ecological divergence and physiological assessment. Such tests inevitably expand our understanding of the intrinsic and extrinsic associations of gorgonian taxa to environmental change from an historical and predictive perspective yielding benefits to conservation assessment and management. 


\section{DISCLAIMER \& AUTHORSHIP STATEMENT}

This thesis was conceived, conducted - including all field, laboratory and data analyses - and written by the author. Notable exceptions are Dr. Xavier Pochon who guided and assisted in molecular work and analyses. Miss Francesca Koethe made light microscope images for Isis hippuris Linnaeus 1758 sclerite measurements. All chapters are modified versions of a series of manuscripts to be submitted for publication on thesis submission, therefore some content overlap will exist between chapters. 


\section{ACKNOWLEDGMENTS}

First and foremost I am indebted to the Sea fans themselves, who through their sheer eloquence connect us to the oceans and wonders of nature; they, are my greatest teachers.

My deepest gratitude to those who saw in me the scientist I did not see in myself until the final months of my degree! Thank you for your patience. They are listed as follows: Professor L. Watling who guided me relentlessly and whose support and encouragement I will forever be indebted, such words are not delivered lightly. Dr. S. Davy who graciously took me on in spite of circumstance and whose support and expertise were invaluable, thank you. To Dr. R. Pyle whose wisdom, encouragement and generosity never cease to amaze me particularly at the $11^{\text {th }}$ hour, I cannot thank you enough!

To Dr. J. Bell and Dr. D. Smith at Victoria University of Wellington and the Coral Reef Research Unit, University of Essex and Operation Wallacea UK respectively, who provided me with the opportunity to research in a place beyond my wildest dreams up to where my presence did not serve them. For the Indonesian staff at ALAM of the Wakatobi Marine National Park, SE Sulawesi, Indonesia, whose selfless efforts and support I will forever be indebted - it is my hope that this modest scientific contribution may assist in the awareness and sustainability of their uniquely diverse marine environment. Specifically, Pak Iwan, Pak Arif, Pak Dd and Pak Azrul for diving and entertainment.

This thesis would not have been completed without the selfless action of Dr. W. Bennet, Ms. T. Dabruzzi, and L. Michie, as well as a special Mahalo to Ms. L. Sta Rosa (Pinky!), C. Corra, H. Webber, Mr. J. Piercy, Dr. K. Muzik, Prof. C. Todd, Dr. C. Kelley, Ms. J. Culp, F. Koethe, D. Lazell, Mo and Imin, J. Williams, Dr. Z. Forsman, Dr. D. Suggett, Dr. S. Donachie, Ms. L. Dueñas, and Mr. R Becker. Sincere gratitude is also extended to Dr. R. Gates and Dr. X. Pochon; it must have been difficult. Endless lab space, time and isotope support were made available from Dr. R. Briggs and Ms. D. Hull, with additional isotope guidance from Dr. J. Padilla-Gamiño, Mrs. C. Ka'apu-Lyons, and Dr. J. Drazen who also provided pilot financial support, thank you very much.

Research in the Wakatobi Marine National Park was conducted in accordance with permits granted by The State Ministry of Research and Technology (RISTEK) to Dr. D. Smith. I am extremely grateful to have been supported by a Victoria University of Wellington (VUW) 
Doctoral Research Scholarship, Exceptional Student award, Submissions Scholarship, the Coral Reef Research Unit, Operation Wallacea, and sponsorship from Mr. P. Duxfield at Cameras Underwater $^{\odot}$ for excellent camera equipment and advice. Appreciation is also extended to the VUW Academic, administrative and dyslexic staff, specifically Mrs. S. de Sain, as well as Mrs. A. Shintani et al. at the University of Hawai'i at Mānoa, USA. Taxonomic support, scholarships and access to museum material were provided by Dr. S. Cairns at the Smithsonian Institute, Dr. E. Rodríguez and Ms. C. LeBeau of the American Museum of Natural History (AMNH), USA, Drs. L. van Ofwegen and B. Hoeksema at the Netherlands Centre for Biodiversity (NCB) Naturalis, to the tune of $\$ 8500$ (USD) with additional facilities from Mr. A. Cabrinovic at the British Natural History Museum, UK, Mrs. Y. Tuti Hermanlimianto at the Indonesian Institute for Sciences (LIPI), Indonesia, and Ms. D. Tracey, Dr. K. Schnabel, Ms. S. Mills and Mr. P. Marriott of the National Institute of Water and Atmospheric Research (NIWA), NZ.

Sanity support, in addition to many mentioned above, includes the friendship and diving with C. Copus, D. Pence and J. Hansen. And last but by no means least, my deep gratitude lies in the silent witness and inspiration from Mrs. H. Forty, G. Weiner, Mr. W. Wilson, Dr. R. Smith, Mrs. R. Smith, Mr. C. Chamberlain, K. Morris, Mr. and Mrs. Y and R Banks-Martin, without which I would not be here.

To my beloved family - Aloha nui loa! 


\section{CONTENTS}

Abstract

iii

Disclaimer \& Authorship Statement

V

Acknowledgments

vi

Contents

viii

List of Tables

List of Figures

List of Abbreviations

xiii

Glossary

Chapter 1: Gorgonian Responses to Environmental Change: Plasticity vs. Adaptation?

1.1 INTRODUCTION

1.2 GORGONIAN ANATOMY

1.3 GORGONIAN CLASSIFICATION

1.4 MORPHOLOGY

1.5 ECOLOGY

1.3 TROPHIC ECOLOGY

1.4 PHENOTYPIC PLASTICITY

1.5 RESEARCH PERSPECTIVE

1.6 AIMS \& OBJECTIVES

Chapter 2: Environmental Gradients Structure Gorgonian Ecology on Coral Reefs in SE Sulawesi, Indonesia.

2.1 INTRODUCTION

2.2 METHODS

1.6 RESULTS

1.8 DISCUSSION

1.9 CONCLUSION

Chapter 2: Environmental Influences on the Indo-Pacific Gorgonian Isis hippuris Linnaeus 1758: Plasticity Capacity or Genetically Fixed? 
Chapter 3: Acclimatory capacity of the Gorgonian Isis hippuris Linnaeus 1758 to environmental change in SE Sulawesi, Indonesia.

$\begin{array}{ll}3.1 \text { INTRODUCTION } & 90\end{array}$

3.2 METHODS 95

$\begin{array}{lr}2.5 \text { RESULTS } & 106\end{array}$

$\begin{array}{ll}2.1 \text { DISCUSSION } & 123\end{array}$

$\begin{array}{ll}2.2 \text { CONCLUSION } & 130\end{array}$

Chapter 4: Gorgonians in the Wakatobi Marine National Park, Indonesia:

What can they tell us about evolutionary processes in environmental change?

4.1 CONTEXTUAL SUMMARY 132

4.2 BIODIVERSITY \& REFUGIA 133

4.3 SYSTEMATICS IN THE SEA 137

4.4 MECHANISMS OF ECOLOGICAL DIVERGENCE 141

$\begin{array}{ll}2.3 \text { CONSERVATION IMPLICATIONS } & 148\end{array}$

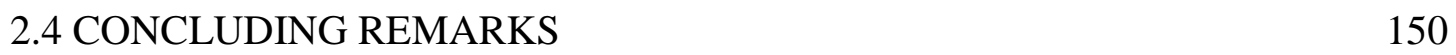

APPENDIX I: Methods for Stable Isotope Analyses 152

A:1 Host and Symbiodinium Separation 152

$\begin{array}{ll}\text { A:2 SEDIMENT } & 152\end{array}$

$\begin{array}{ll}\text { A:3 PLANKTON } & 153\end{array}$

$\begin{array}{lr}\text { REFERENCES } & 154\end{array}$ 


\section{LIST OF TABLES}

\section{Chapter 2: Environmental Gradients Structure Gorgonian Ecology on Coral}

Reefs in SE Sulawesi, Indonesia.

2.1 Environmental characteristics of the four study sites in the WMNP 31

2.2 Gorgonian species inventory and abundance 34

2.3 CAP analyses results assessing gorgonian species assemblages 41

2.4 SIMPER analysis results for all gorgonian species 43

2.5 SIMPER analysis results for zooxanthellate species 44

2.6 SIMPER analysis results for azooxanthellate species 45

Chapter 3: Environmental Influences on the Indo-Pacific Gorgonian Isis

hippuris Linnaeus 1758: Plasticity Capacity or Genetically Fixed?

3.1 Environmental characteristics of study sites within the WMNP 68

3.2 Isis hippuris morphological trait summary table 73

3.3 ITS2 Accessions of octocoral outgroups $\quad 75$

3.4 Isis hippuris ITS2 haplotype sequence view $\quad 77$

3.5 AMOVA of genetic structure between sites within the WMNP 78

Chapter 4: Acclimatory Capacity of the Gorgonian Isis hippuris Linnaeus 1758

to Environmental Change in SE Sulawesi, Indonesia.

4.1 Characteristic environmental variables at optical equivalent depths $(\zeta) \quad 96$

$\begin{array}{ll}4.2 \text { Accessions of comparative Symbiodinium clades } & 104\end{array}$

4.3 Isis hippuris test colony annual mortality and survival 106

4.4 Isis hippuris macro-morphological (colony level) traits at Ridge 1

4.5 Isis hippuris macro-morphological (colony level) traits at Sampela 108

$\begin{array}{ll}4.6 & \text { Isis hippuris micro-morphological traits } \\ & 109\end{array}$

$\begin{array}{ll}4.7 \text { Isis hippuris optical parameters } & 110\end{array}$

4.8 PERMANOVA of Isis hippuris test colony phenotypic traits 112

4.9 Phenotypic trait correlation table of Isis hippuris multivariate traits $\quad 121$ 


\section{LIST OF FIGURES}

Chapter 1: Gorgonian Responses to Environmental Change: Plasticity vs. Adaptation?

1.1 Schematic diagram of the gorgonian anatomy 3

1.2 Gorgonian polyp morphology 4

1.3 Gorgonian comparative axis cross-section structure 5

$\begin{array}{ll}1.4 \text { Octocoral phylogeny based on morphological characters } & 7\end{array}$

$\begin{array}{ll}1.5 \text { Reaction norms } & 19\end{array}$

Chapter 2: Environmental Gradients Structure Gorgonian Ecology on Coral Reefs in SE Sulawesi, Indonesia.

2.1 Location map of the Wakatobi Marine National Park, Indonesia 28

2.2 Gorgonian species richness 33

2.3 Constrained and unconstrained ordinations of gorgonian assemblages $\quad 38$

2.4 Zooxanthellate and azooxanthellate gorgonian species richness 39

2.5 Constrained and unconstrained ordinations of zooxanthellate gorgonian $\begin{array}{ll}\text { assemblages } & 41\end{array}$

2.6 Constrained and unconstrained ordinations of azooxanthellate gorgonian assemblages $\quad 46$

2.7 Gorgonian family abundance $\quad 47$

2.8 DISTLMforward models for the best explanatory drivers of variability 48

Chapter 3: Environmental Influences on the Indo-Pacific Gorgonian Isis hippuris Linnaeus 1758: Plasticity Capacity or Genetically Fixed?

3.1 Isis Linnaeus 1758 comparisons 61

3.2 Isis hippuris morphotypes within the WMNP, Indonesia 67

3.3 Isis hippuris morphological trait measurements 69

3.4 Sclerite diversity of Isis hippuris within the WMNP, Indonesia 71

3.5 Unconstrained and constrained ordinations of Isis hippuris character traits $\quad 76$

3.6 Isis haplotype network with ITS2 RNA predicted secondary structure 79

3.7 Phylogram of the ITS2 region from twenty Octocoral taxa in GenBank and Isis haplotypes within the WMNP $\quad 82$ 
Chapter 4: Acclimatory Capacity of the Gorgonian Isis hippuris Linnaeus 1758

to Environmental Change in SE Sulawesi, Indonesia.

4.1 Isis hippuris phenotypic module integration models 94

4.2 Comparative test site location map within the WMNP, Indonesia 95

4.3 Isis hippuris reciprocal transplant experimental design 97

4.4 Isis hippuris morphological trait measurements 99

4.5 Isis hippuris colonies bearing externally brooded eggs, and polyp depth 105

4.6 CAP ordinations of all Isis hippuris phenotypic traits between sites 111

4.7 CAP ordination and reaction norms of Isis hippuris morphological traits 113

4.8 CAP ordination and reaction norms of Isis hippuris polyp dimensions 115

4.9 CAP ordination and reaction norms of Isis hippuris sclerite dimensions $\quad 116$

4.10 CAP ordinations and reaction norms of the Isis hippuris holobiont optical $\begin{array}{ll}\text { parameters } & 117\end{array}$

4.11 Phylograms of Symbiodinium clades for COX1, ITS2 and psbA regions 119

4.12 Phenotypic module integration modules of Isis hippuris morphotypes 122

\section{Chapter 5: Discussion}

5.1 Indo-Pacific biogeographic distribution of shallow and mesophotic gorgonian families

5.2 Comparative axis structure within the Isididae 138

5.3 Comparative gorgonian phylogenetic reconstruction with the Isididae $\quad 140$

5.4 Sliding scale of phenotypic evolution 142

5.5 Isis hippuris morphotypes across bathymetry within the WMNP, Indonesia 143

5.6 Phylogram of Symbiodinium clades within Isis hippuris at source depth $\quad 145$

$\begin{array}{ll}5.7 \text { Isis hippuris holobiont stable isotope analyses } & 147\end{array}$ 


\section{LIST OF ABBREVIATIONS}

$* a_{\mathrm{chl} a}-$ Chlorophyll $a$ specific absorption coefficient

AMNH - American Museum of Natural History

BNHM - British Natural History Museum

BPBM - Bernice Pauahi Bishop Museum (USA)

CAP - Canonical analysis of principal coordinates

Chl $\boldsymbol{a}$ - Chlorophyll $a$

COX1 - Mitochondrial-encoding cytochrome oxidase subunit1 region

F - Fluorescence

Fm' - Maximum yield of chlorophyll fluorescence in a light-adapted state/ambient light

$\Delta \mathbf{F} / \mathbf{F m}$ ' - Light-adapted quantum yield of PSII (below)

ITS - Internally transcribed spacer regions of ribosomal DNA

NIWA - National Institute of Water and Atmospheric Research (New Zealand)

nMDS - Non-metric multidimensional scaling

NMNH - National Museum of Natural History (formerly the USNM, USA; see below)

PAM - Pulse amplitude modulation

PAR - Photosynthetically active radiation

PERMANOVA - Permutational ANOVA (analyses of variance) 
$\boldsymbol{p s} \boldsymbol{b A}$ - Chloroplast plastid-coding $p s b A$ minicircle which encodes the D1 protein of photosystem II

PSII - Photosystem II (two)

RTEs - Reciprocal Transplant Experiments

SIMPER - Similarity percentages

USNM - United States National Museum (now the NMNH, USA; see above)

$\boldsymbol{V}_{\boldsymbol{E}}-$ Environmental variance

$V_{\text {error }}$ - Residual Components

$V_{G}-$ Genetic variance

$V_{G, A}-$ Additive genetic variance

$\boldsymbol{V}_{\boldsymbol{G}, \boldsymbol{D}}-$ Dominant genetic variance

$\boldsymbol{V}_{\boldsymbol{G} \boldsymbol{x} \boldsymbol{E}}-$ Genetic and environmental interaction variance

$V_{G I}-$ Epistatic variance

$\boldsymbol{V}_{\boldsymbol{P}}$ - Phenotypic variance 


\section{GLOSSARY}

Absorbance $(\boldsymbol{D})$ - Measure of the capacity of e.g., chlorophyll to absorb light of a specific wavelength

Absorption/Absorptance $(\boldsymbol{A})$ - Light fraction absorbed

Adaptation - The products of natural selection by which an organism may become better suited to its environment for a specific function(s)

Adaptive - Having the capacity for adaptation (above)

Adaptive plasticity - Where plasticity is considered beneficial and maintained by selection

Additive genetic variance $\left(\boldsymbol{V}_{\boldsymbol{G}, \boldsymbol{A}}\right)$ - Deviation from the population mean phenotype due to additive allele effects (substituting one allele for another) at a given locus or the multiple loci of a polygenic trait (below)

Anastomose - Forming a network (in the context of colony morphology)

Anthocodia - The distal part of a polyp, bearing the mouth and tentacles; can be retracted within the calyx, stem, branch or cortex

Anthostele (Calyx) - The proximal, rigid part of polyps in some octocoral species, often stiffened by sclerites. In some cases the anthocodia is withdrawn into the anthostele

Arborescent - Tree-like colonies possessing a stem

Axial sheath - Part of the colonial coenenchyme immediately surrounding the axis, usually delimited by the longitudinal stem canals and characterised by sclerites commonly different in form from those of the overlying coenenchyme

Axis - Central, longitudinal supporting structure which can consist of either, 1) an inner central horny (gorgonin) chord with or without calcareous material (loculi), 2) scleritic inner medulla and outer coenenchyme, or 3) non-scleritic central axis which may be hollow or 
cross-chambered

Axis cortex (Cortex) - Tissue layer surrounding the central part of the axis; either the coenenchyme surrounding the medulla or the horny layer surrounding the central chord

Bushy - Colonies with abundant branches typically in all directions

Canal (Longitudinal) - Longitudinal partitions of the gastrovascular cavity of the polyp running longitudinally along the central axis, often nested within axial groves

Canalisation - The reduced sensitivity (sensu fixed) of a phenotype to changes or perturbations in the underlying genetic and nongenetic factors that determine its expression

Capstan - Sclerite: rod with two whorls of tubercules or wards and terminal tufts

Central chord (Core) - The central part of a Holaxonian axis, consisting solely of horny material or horny material with varying permeations of calcareous material (loculi). Can be hollow and cross-chambered in some families (see Medulla)

Club - Monaxial (single axis) sclerites enlarged at the head, and tapered at the opposite end, the handle

Coenenchyme - The colonial soft tissue between the polyps, consisting of the mesoglea usually containing sclerites and penetrated by the network of solenia and gastrodermal canals

Cortex - Tissue layer surrounding the central part of the axis; either the coenenchyme surrounding the medulla or the horny layer surrounding the central chord.

Cross - Stellate sclerite with four rays in a single plane

Crown (Collaret) - The ring of transversely placed, usually bow-shaped sclerites encircling the anthocodia below the tentacles

Diving-PAM - A diving-pulse amplitude modulation fluorometer measuring the effective quantum yield $(\Phi)$ of photochemical energy conversion during photosynthesis 
Dominant genetic variance $\left(V_{G, D}\right)$ - Deviation from the population mean phenotype due to interactions between alleles at the same locus

Dumb-bell - Sclerite with two nearly spherical, warty heads and a distinct waist that is longer than in the double sphere

Encrusting - Colony morphology consisting of a thick fleshy layer covering the substrate

Environmental variance $\left(\boldsymbol{V}_{\boldsymbol{E}}\right)-$ Phenotypic variance among individual members of a population due to environmental effects

Epigenetic - Resulting from external rather than genetic influences, whereby modification in gene expression/function are independent of the DNA sequence. Epigenetic influences can become heritable and fixed (through genetic assimilation) over time

Epistatic interaction variance $\left(\boldsymbol{V}_{\boldsymbol{G I}}\right)$ - Genetic variance due to epistasis (below)

Epistasis - The expression of a gene is modified by the presence of one or more "modifier genes' from different loci.

Evolutionary capacitance - The storage and release of genetic information (variation), typically leading to fixation through genetic assimilation as a consequence of epigenetic heritability

Flabellate - Fan-shaped (arborescent)

Gastric cavity (Gastrovascular cavity; Coelenteron) - Interior space of a polyp

Gastrodermal canals - Wide, endodermal-lined canal connected with the narrower solenia and originating from them

Genetic accommodation - A process where a phenotype is originally produced in response to either a mutational or environmental stimulus (genetic assimilation in the latter; below)

Genetic assimilation - A process where a phenotype originally produced in response to environmental change later becomes genetically encoded typically through epigenetic xvii 
heritability. Sensu stricto process of phenotypic evolution by genetic accommodation (above)

Genetic variance $\left(\boldsymbol{V}_{\boldsymbol{G}}\right)$ - Phenotypic variance among individual members of a population due to genetic effects

Genetic and environmental interaction variance $\left(V_{G x E}\right)$ - Genotype by environment interaction whereby genotypes differentially respond to their environment

Gonads - Reproductive cells along the septa within the gastrovascular cavity

Gorgonin - Horny proteinaceous material forming with calcareous (loculi and/or sclerites) material of the inner and/or outer layers of the central axis

Hermatypic - Reef-building corals, typically depositing aragonite structures contributing to or the basis of coral reef development e.g., most Scleractinian corals

Holobiont - Biological unit including the host and its microbial associate communities

Integration - Characters (phenotypic traits or modules) behaving as a unit with integration manifested as coordinated character change in ontogeny, phylogeny, space, time, magnitude or direction

Internode - Hard, calcareous segment of the jointed axis (e.g., Melithaeidae, Isididae)

Intraspecific variation - Any differences among individuals of a single species.

ITS2 cladal type - Genetic variant of the ITS2 region below that of a clade and currently unresolved taxonomically

Loculi (Loculus) - Calcified or fibre-filled space in the holoxonian axis, especially in Plexauridae, appearing crescentic or lenticular in cross section

Medulla - Central supporting structure of the Scleraxonia consisting of densely packed sclerites, gorgonin and occasional longitudinal canals. Surrounded by the coenenchyme (see 
Axis, Central Chord)

Mesenteries (Septa/um) - Eight thin, radial and longitudinal, non-calcareous partitions joining the pharynx to the body wall and dividing the polyp gastrovascular cavity. Each septum bears a longitudinal retractor muscle

Mesenteries filaments (Septal filaments) - The thickened convoluted edges of the mesenteries (septum) below the pharynx. The two mesenteries opposite the siphonoglyph are long and heavily flagellated

Modularity - Degree to which a system's components can be separated and recombined; thus organisms are considered to consist of phenotypic modules

Monophyletic - A group of organisms descended from a common evolutionary ancestor or ancestral group, particularly one that is not shared with any other group

Multiplanar - Branched colonies in which the branches grow in several planes

Neck zone (Introvert) - Soft, thin-walled basal section of the anthocodia below the tentacles bearing little or no sclerites. Permits introversion of the anthocodia into the anthostele (calyx)

Node - The flexible horny (gorgonin) segment of a jointed axis (see internode)

Oral disc - Area of the polyp immediately surrounding the mouth and formed by the inner basal parts of the tentacles

Pharynx - Tubular section of the digestive system connecting the mouth and the gastrovascular cavity; possesses one or two flagellated grooves (siphonoglyphs)

Phenotype - The set of observable traits of an individual due to its interaction of its genotype with the environment

Phenotypic plasticity - Environmentally induced changes in an organism's phenotype within its lifetime; a specific form of intraspecific variation (see above). 
Phenotypic variance $\left(\boldsymbol{V}_{\boldsymbol{P}}\right)$ - Variance within a quantitative phenotypic trait (see additive, dominant, epistatic, genetic, environmental, and genetic and environmental interaction)

Polyphyletic - A group of organisms derived from more than one evolutionary ancestor or ancestral group, therefore not suitable for placing in the same taxon

PSII - Photosystem II (two); a photosystem reaction centres/protein complex that uses light energy for the splitting of water through oxidation in photosynthesis

Pinnules - The lateral, hollow processes arranged in two opposite rows along each tentacle

Planar - Arborescent branching colonies where branches generally grow in a single plane

Plasticity - Environment-dependent phenotype expression

Pleiotropy - The influence of a single gene on several seemingly unrelated phenotypic traits

Point(s) - Eight longitudinal rows of chevroned sclerites around the distal part of the anthocodia, superposing the crown if present

Polygenic - Phenotypic trait controlled or the product of two or more genes and its environment (see additive genetic variance)

Polyp (Zooid) - Any individual within a (octocoral) colony, which may be monomorphic (possess single polyp type) or consist of more than one type e.g., autozooids and siphonozooids (e.g., Coralliidae)

Polyphenic trait (Polyphenism) - A trait which gives rise to multiple, distinct phenotypes from a single genotype due to differential environmental conditions

Radiates - Sclerites with symmetrically radiating processes in a single or multiple planes

Reaction norms (Norms of reaction) - A single genotypes phenotypic expression as a function of environmental variation. 
Reticulate - Branching colonies where branches anastomose forming net-like structures

Sclerite - Calcareous skeletal element present in or on the Octocoral soft tissue matrix or axial composition

Septa (Septum, Mesenteries) - Eight thin, radial and longitudinal, non-calcareous partitions joining the pharynx to the body wall and dividing the polyp gastrovascular cavity. Each septum bears a longitudinal retractor muscle

Siphonoglyph (Sulcus) - Strongly ciliated groove extending down one side of the pharynx

Solenia (Solenium) - Narrow endodermal canal lined with gastrodermis within the coenenchyme, forming a network by interconnecting the gastric cavities of the polyps and larger canals

Spicule - Skeletal element of non-cnidarian taxa (e.g., Porifera), often confused with sclerite

Spindles - Monaxial (single axis) sclerites that are straight or curved and pointed at both ends

Symbiodinium Freudenthal 1962 (Zooxanthellae) - Unicellular dinoflagellate free-living or endosymbiotic alga. The largest group of endosymbiotic dinoflagellates

Synapomorphic - Of a character trait that evolves once in the common ancestor (and not in its ancestors) of two or more lineages, themselves possibly exhibiting further modified versions of that trait

Residual components $\left[\boldsymbol{V}_{\text {error }}\right]$ - Phenotypic variance accounted for by developmental noise, bethedging, behavioural or other unaccountable factors

Zooxanthellar/e (Symbiodinium) - Colloquial name for Symbiodinium Freudenthal 1962

\section{Glossary Bibliography}

Bayer FM, Grasshoff M, Verseveldt I eds. (1983) Illustrated trilingual glossary of morphological and anatomical terms applied to Octocorallia. E. 1. Brill/Dr. W. Backhuys, Leiden. pp.75, 20 pls.

DeWitt TJ, Scheiner SM (2004) Phenotypic variation from single genotypes. In: Phenotypic Plasticity: 
Functional and conceptual approaches (eds. DeWitt TJ, Scheiner SM), pp. 1-9. Oxford University Press, New York.

Gotthard K, Sören Nylin S. (1995) Adaptive Plasticity and Plasticity as an Adaptation: A Selective Review of Plasticity in Animal Morphology and Life History .Oikos, 74(1): 3-17.

Hageman SJ, Bayer MM, Todd CD (1999) Partitioning phenotypic variation: genotypic, environmental and residual components from bryozoan skeletal morphology. J. Nat. Hist. 33(11): 1713-1735

Johnson C (1976) Introduction to natural selection. University Park Press, Baltimore. pp. 213

Roth LV (1996) Cranial Integration in the Sciuridae. Amer. Zool. 36: 14-23

Stachowitsch M. (1992) The invertebrates: an illustrated glossary. Wiley-Liss, Inc., New York. pp. 676

West-Eberhard MJ. (2003) Developmental Plasticity and Evolution. Oxford Univ Press, New York. 


\section{CHAPTER 1: GORGONIAN RESPONSES TO ENVIRONMENTAL CHANGE: PLASTICITY VS. ADAPTATION?}

\subsection{INTRODUCTION}

Gorgonian corals (Cnidaria: Anthozoa: Octocorallia) are conspicuous, diverse and often dominant components of intertidal and subtidal benthic marine environments; most notably tropical shallow reef, deep sea, and mesophotic habitats (Wirshing et al. 2005, McFadden et al. 2010a, Cerrano et al. 2010). Gorgonians are modular, suspension feeding colonial sessile organisms defined primarily by a semi-rigid scleroproteinaceous (gorgonin) axis with varying amounts of calcification (Bayer 1961, Grasshoff 1999, Sánchez et al. 2003a). Originally classified under the order Gorgonacea (now taxonomically obsolete), Bayer (1981) included gorgonians within the Octocorallia order Alcyonacea on the basis of intermediate forms obscuring any definitive morphological boundaries. Thus, gorgonians remain in a state of taxonomic confusion despite being of ecological (Fabricius \& De'ath 2004), commercial (Grigg 2002), climatic (Thresher et al. 2010), evolutionary (Sánchez 2004), pharmaceutical (Bayer et al. 1974, Bordeleau et al. 2006, Susilaningsih et al. 2010), and conservation importance (Dayton 2003, Linares et al. 2008). Many gorgonians have been used as conservation 'flagship' species (Tinsely 2005, Linares et al. 2008, Cerrano et al. 2010), being ecologically diverse, long-lived engineering taxa that maintain habitat heterogeneity and provide secondary space to other organisms, thereby enhancing ecosystem function (Cerrano et al. 2010, Mumby et al. 2010). Irrespective of their ecological diversity and global distribution, the greatest paucity of information however, exists in the Indonesian Archipelago (Tomasik 2004), a surprising reflection particularly given that zooxanthellate gorgonians are often one of the primary space occupants of tropical reefs (McFadden et al. 2006).

Gorgonian responses to their environment are intriguingly complex. Their population demographics are principally driven, together or in part, by environmental factors including substrate, light, temperature, sedimentation, salinity, current regime and flow rates (Bayer 1981, Fabricius \& Alderslade 2001). In addition, biotic factors such as competition, predation, symbioses, reproduction, settlement and developmental properties provide local scale refinement. These factors have been shown to induce intra- and inter-specific morphological variability (West 1997, 1998, Linares et al. 2008), habitat selection and colony orientation (Grigg 1972, Sánchez et al. 2003a). Anatomical and behavioural adaptations, which include polyp expansion, chemical or nematocyst complement, colony dynamics, branching morphology, sclerite type and morphology, as well as photoacclimation, zooxanthellae density 
and content, are all responses to reef life. Yet, what stands this group apart from other marine metazoans, with the exception of perhaps Porifera (Bayer 1961, Shearer et al. 2002), is the enormous degree of variability as yet largely unexplored. Whether such variability is the result of phenotypic plasticity, therefore adaptive, or an adaptation potentially leading to incipient speciation remains to be seen. Nevertheless, to investigate evolutionary processes and create effective conservation strategies, it is essential to define species and species boundaries. However, such taxonomic resolution is often confounded by considerable phenotypic variability, cryptic and sibling taxa (Knowlton 1992), and lack of gorgonian research primarily due to difficulties in field identification (Fabricius \& Alderslade 2001). In addition, molecular markers such as mitochondrial DNA (mtDNA) used to delineate species and/or taxonomic groups are highly conserved in Cnidaria revealing little or no taxonomic variation (France et al. 1996, Shearer et al. 2002). Thus, the 'Species Problem' is exemplified and of fascinating complexity in gorgonians. Nonetheless, with a modular, clonal nature and advancements in molecular markers (e.g. Conception et al. 2008, McFadden et al. 2010a), gorgonians provide an innovative platform from which to study the evolution of environmentally plastic or dependent characters (Gotthard \& Nylin 1995) and subsequent modes of speciation.

\subsection{GORGONIAN ANATOMY}

Colonial, polypoid and sessile, gorgonian octocorals are characterised by polyps bearing eight pinnate tentacles, eight mesenteries dividing the gastrovascular cavity, and nematocyst (collectively 'cnidae') complement (Figure 1.1: Bayer 1961, Berntson 1998). What defines a gorgonian coral specifically is the division of the coenenchyme - the tissue between and containing the polyps, gastrovascular canals, sclerites and solenia (see Figure 1.1) - into the outer cortex and inner axial medulla (as in the Scleraxonians Figure 1.3a) or a central axis with or without a central cortex (Figure 1.3c,b). This tissue division may or may not give rise to the archetype arborescent morphology of a gorgonian and places emphasis on structural - functional optima of resource acquisition in particular environments. For example, encrusting zooxanthellate taxa in high light and hydrodynamic regimes, and tall flexible arborescent colonies perpendicular to the prevailing water currents. Ultimately it is the central or inner axial layer consisting of varying levels of calcareous and/or gorgonin (proteinaceous) material that lend gorgonians their name. 


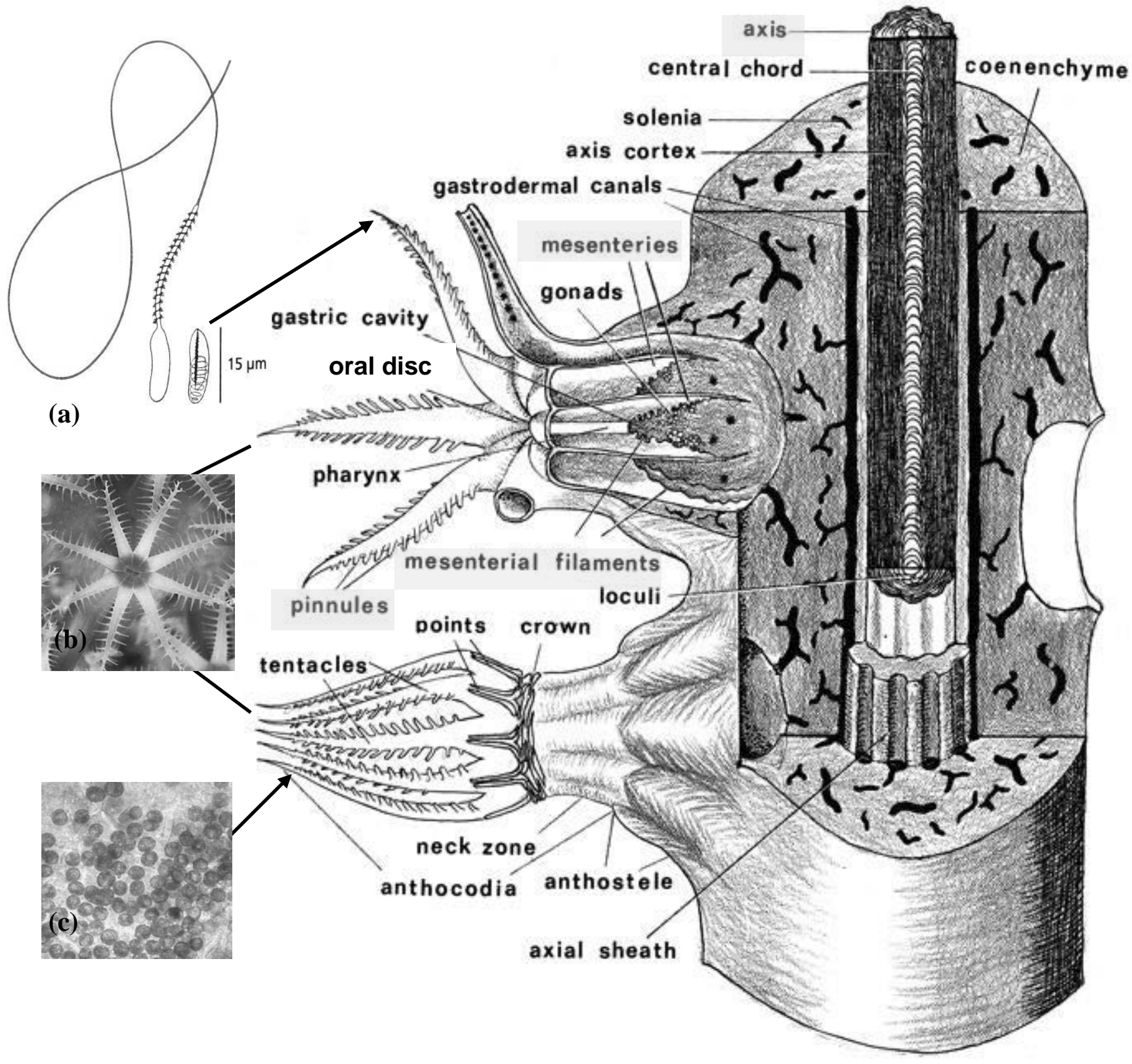

Figure 1.1 Schematic diagram of gorgonian anatomy modified from Bayer et al. (1983), with (a) Octocoral nematocyst capsule (modified from Grasshoff \& Bargibant 2001), (b) polyp overhead view (taken by Eco-Divers 2008), and (c) endosymbiont dinoflagellates (zooxanthellae) within the polyp gastrodermis (source Hoegh-Guldberg). Yellow highlighted traits are particularly characteristic of gorgonian corals. Glossary of terms provided on page xvi.

The coenenchyme growth leads to the continual addition of polyps that may retract entirely into the coenenchyme (Figure 1.2a), into low or high calyces (Figure 1.2b,c) or contract through hydrostatic deflation into low mounds or tall scleritic polyp structures (Figure 1.2d,e). The latter two cases do not reinforce the anthostele and thus are not termed calyces (Stachowitsch 1992). 


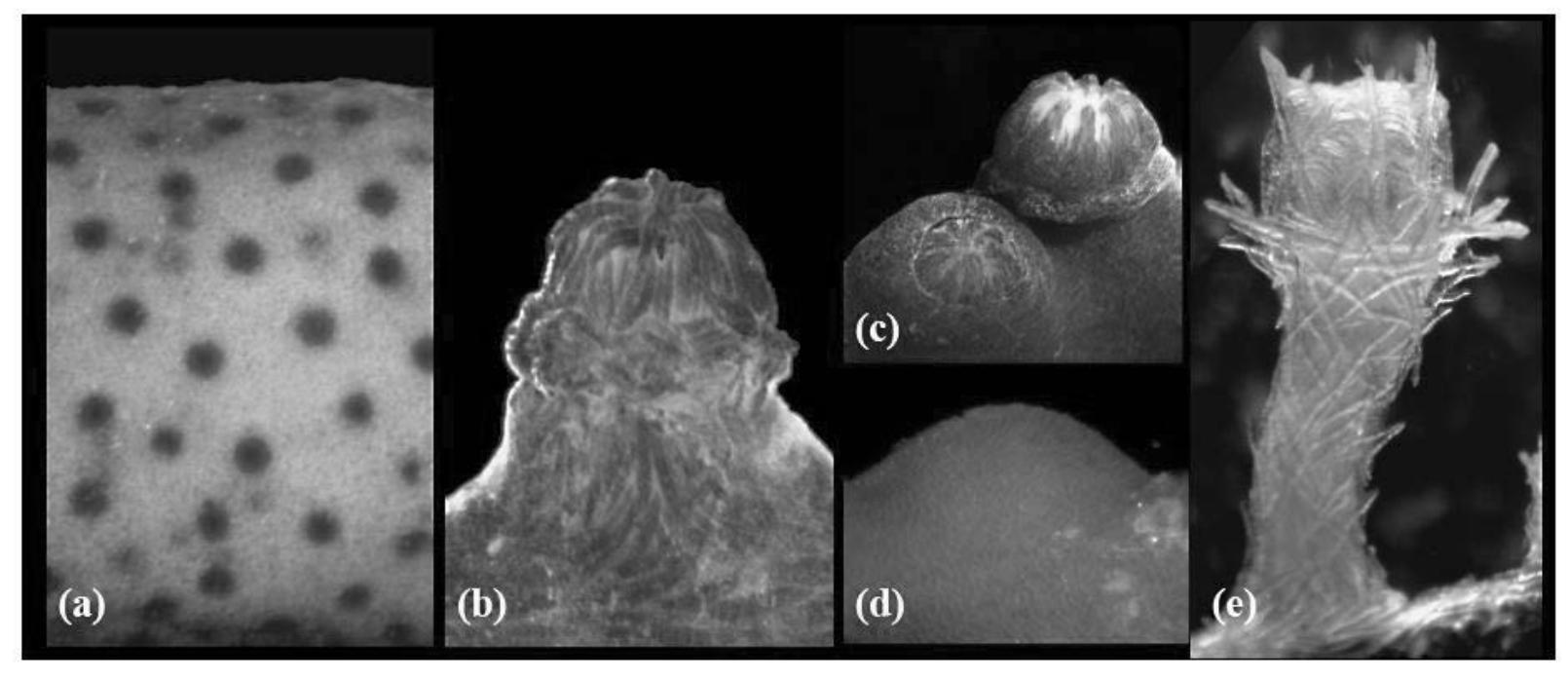

Figure 1.2 Gorgonian polyp morphology. Retractile polyps of (a) Isis hippuris Linnaeus 1758 completely into the coenenchyme, (b) Astrogorgia Verrill 1868 into a high calyx, and (c) in to a low calyx plus partial expansion. Non-retractile but contractile polyps of (d) Verrucella MilneEdwards \& Haime 1857, and (e) Acanthogorgia Gray 1857 (photography by Rowley 2009).

Gorgonian polyps consist of three thin layers: the mesoglea, a gelatinous fibrous matrix also containing amoeboid and scleroblast cells, sandwiched between the outer epidermis and inner gastro- or endodermis cellular layer lining the mesenteries, pharynx, gastric cavity and tentacles (Stachowitsch 1992, Fabricius \& Alderslade 2001). The eight mesenteries divide and increase the surface area of the gastric cavity through the pharynx to the eight pinnate tentacles, themselves mesentery extensions. The free inner edge of each mesentery below the pharynx is thickly lined (mesentery filament) and varies in function with two flanking the longitudinal siphonglyph or sulcus, which through ciliary action beats water through the polyp into the solenia or canals to the rest of the colony. In zooxanthellate species the dinoflagellate endosymbionts are present within the gastrodermal cells or within vacuoles in the gastric cavity (Fabricius \& Alderslade 2001).

Nematocysts are only produced by cnidarians as key mechanisms of defence and offence (Fautin 2009). Gorgonian nematocysts are typically in the tentacles, if present at all, with octocoral cnidae somewhat pitiful compared to that of other cnidarians (e.g., Hydrozoa, Scyphozoa, Cubozoa) consisting of a single type (Figure 1.1a) out of the thirty currently recognised for this phylum (Fautin 2009). Irrespective, gorgonians have continued to persist since at least the Lower Ordovician (Lindstrom 1978, Bengtson 1981, Cope 2005) despite their relative simplicity. It is not unreasonable to suggest that their current poor cnida content, diversity and 

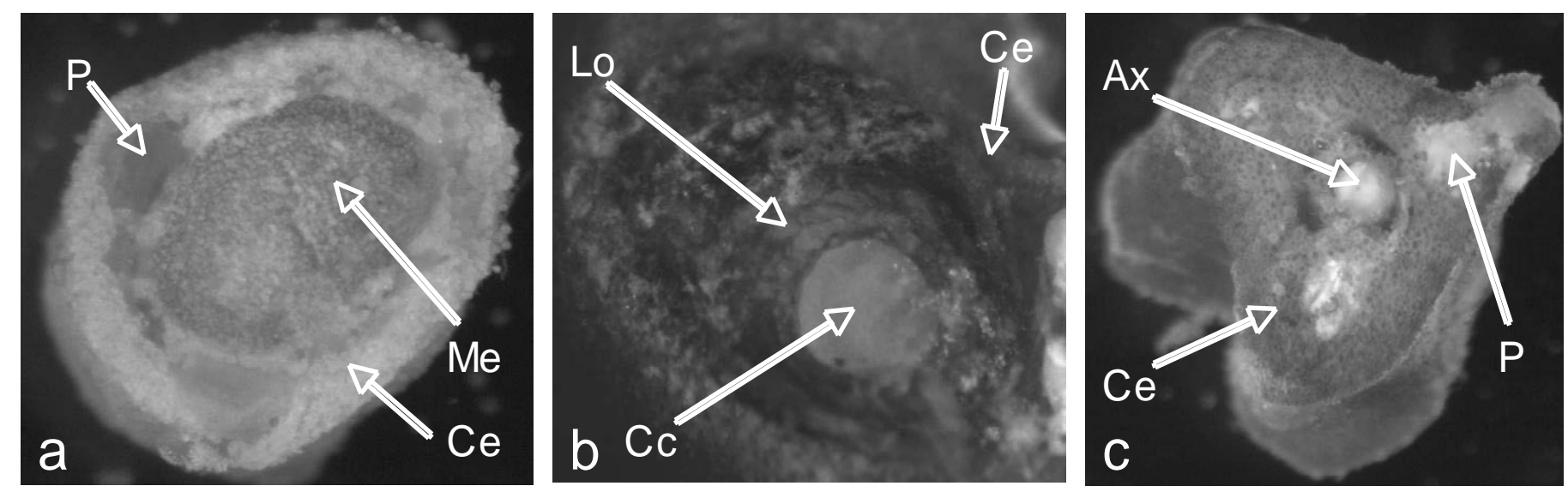

Figure 1.3. Gorgonian comparative axis cross-section structure. (a) Scleraxonian axis crosssection showing coenenchyme, polyp and central medulla (Annella reticulata Ellis \& Solander 1786, $6 \mathrm{~mm}$ ). (b) Holaxonian axis cross-section showing loculus, central core and coenenchyme (Astrogorgia cf. dumbea Grasshoff 1999, $7 \mathrm{~mm}$ ). (c) Calcaxonian axis cross-section showing coenenchyme, axis and polyp (Viminella sp. Gray 1870, $4 \mathrm{~mm}$ ). Ax = Axis, Cc = Central core, $\mathrm{Ce}=$ Coenenchyme, Lo = Loculus, $\mathrm{Me}=$ Medulla, $\mathrm{P}=$ Polyp. Images: Wakatobi Marine National Park, Indonesia, 5 - 15 m depth (Rowley) 2009, 2010.

potency may be due to increasing redundancy over time through a greater chemical defense battery, which may or may not have evolved as a consequence of strong associations with their microbiota.

\subsection{GORGONIAN CLASSIFICATION}

Gorgonians (sea fans and sea whips) are within the subclass Octocorallia (or Alcyonaria), which take a basal position within Anthozoa, itself basal within the phylum Cnidaria (Bridge et al. 1992). Octocorallia are a clearly defined monophyletic group based on both their molecular and anatomical characters (France et al. 1996, Bayer 1961, Berntson et al. 1998, 2001, McFaddenet al. 2010a).

Gorgonian taxonomy began almost four centuries ago, being originally described and classified as 'marine plants' (Rumphius 1741, Bayer 1959, Grasshoff 2001), with eventual classification based on colony morphology and the shape and arrangement of calcareous sclerites found within the polyps and coenenchyme (Kükenthal 1919, Bayer 1981, Grasshoff 2001, Vargas et al. 2010). Bayer $(1961,1981)$ further confirmed gorgonian delineation on the basis of axis mineralogy (Figure 1.3a-c). Therefore, through comparative morphology gorgonians currently comprise the suborders, Holaxonians and Calcaxonians Grasshoff 1999 and the group Scleraxonians Studer 1887 within the order Alcyonacea (Bayer 1981, Figure 1.4). The Scleraxonian 'group' however, 
is not strictly a suborder due to several intermediate forms and the likelihood of families evolving from numerous separate evolutionary lines (Fabricius \& Alderslade 2001). Nonetheless, Scleraxonians typically possess a scleritic inner medulla with varying low levels of gorgonin. The Holaxonians have, with one exception (Keroeididae Kinoshita 1910), a whole horny axis supported by non-scleritic calcareous material. The relatively recent (Grasshoff 1999) Calcaxonians are delineated by a solid non-scleritic calcite or aragonite axis, which may alternate or fuse with gorgonin fibers (Bayer 1981, Fabricius \& Alderslade 2001; Figure 1.3). However, hollow axial members exist (Watling \& France 2011, Alderslade \& McFadden 2012)!

Curiously, molecular studies confirm Octocorallia monophyly but lack phylogenetic support for such morphology-based sub-ordinal groupings within the Alcyonacea (reviewed by McFadden et al. 2010a, Figure 1.4). Sánchez et al. (2003a) using partial 16S mitochondrial rDNA, complete 18S nuclear rDNA sequences (from France et al. 1996 \& Berntson et al. 2001 respectively), INDELS (insertions-deletions) and predicted secondary structure of the 1su-rRNA (16S) revealed two main branching gorgonian clades; Calcaxonia and Alcyoniina-Holaxonia, with Scleraxonians present in both groups. Moreover, McFadden et al. (2006) using the mitochondrial protein-coding regions, $m s h 1$ (now $m t M u t S$ ) and ND2 on all Octocorallia subgroups further supported the findings of Berntson et al. (2001), revealing three well-supported clades (cf. Figure 1.4). Gorgonian phylogeny therefore, typically recognises two groups, with further separation of deep and shallow water clades within Calcaxonians (McFadden et al. 2006, Pante et al. 2012), and Scleraxonians being polyphyletic (Sanchez et al. 2003a). Furthermore, mapping morphological characters onto molecular-derived phylogenies revealed synapomorphic and homoplasious characters, for example axial structure and surface sclerite morphology respectively in Caribbean Holaxonians (Sanchez et al. 2003b). Such phylogenetic research, though informative, fails to satisfactorily resolve at the subordinal or family-level (McFadden et al. 2010a) due to such considerable overlap and lack of reliable morphological characters (Wirshing et al. 2005), further confounded by remarkably slow evolving mitochondria in the Anthozoa (Shearer et al. 2005). However, Octocoral mitochondria contain mshl, a homolog of the bacterial mismatch repair gene MutS (Pont-Kingdon et al. 1995, 1998), synapomorphic for the subclass and twice as variable as other traditionally used mitochondrial markers (France \& Hoover 2001, van der Ham et al. 2009, McFadden et al. 2010a). Yet, levels of $m s h 1$ inter- and intraspecific variation have been shown to differ between genera (McFadden et al. 2010b), and combined use of $m s h 1$, mitochondrial and/or nuclear genes seldom leads to increased taxonomic resolution (e.g., Sánchez et al. 2003b, Wirshing et al. 2005, Herrera et al. 2010). Furthermore, studies utilising 


\title{
Octocorallia/Alcyonaria
}

\author{
-Alcyonacea (Soft Corals \& Gorgonians) \\ - Calcaxonians (Gorgonians) \\ - Holaxonians (Gorgonians) \\ - Scleraxonians (Gorgonians) \\ - Alcyoniinans (True Soft Corals) \\ - Stoloniferans (Stolon Corals) \\ - Helioporacea/Coenothecalia (Blue Corals) \\ - Pennatulacea (Sea Pens \& Sea Pansies)
}

Figure 1.4 Octocoral phylogeny based on morphological characters currently in use based on Bayer (1981) and Fabricius \& Alderslade (2001).

nuclear rDNA internal transcribed spacers (ITS) reveal conflicting results both between and within gorgonian species (Aguilar \& Sánchez 2007, Sánchez et al. 2007, Dueñas \& Sánchez 2009, Gutiérrez-Rodríguez et al. 2009, cf. Calderón et al. 2006). Such multicopy markers (tandem repeats of transcription units within a cell) are subject to intragenomic variation (variability between such tandem repeats), controversial in the validity of the results ( $c f$. Coleman 2003, 2007, 2009). The predicted RNA secondary structure is more conserved and therefore, generally considered more phylogenetically informative particularly in the cases of Caribbean and deep-sea gorgonians (Aguilar \& Sánchez 2007, Sánchez et al. 2007, Sánchez \& Dorado 2008, Dueñas \& Sánchez 2009). Nonetheless, Concepcion et al. (2008) revealed cryptic species delineation using the single-copy marker SRP54 (>33\% variation $c f$. $<10 \%$ in mtDNA ND2 \& 6) within Carijoa riisei Duchassaing \& Michelotti 1860, thus informative intraspecific variation without the caveats of intragenomic variation. In contrast, Watling \& France (2011) discovered large numbers of indels (sequence insertions and deletions) rendered the sequences inoperable for phylogenetic analyses in the Keratoisidinae.

As with morphological characteristics the choice and utility of molecular marker(s) appears subjective relative to their resolution efficiency (Wirshing et al. 2005) and target taxon. What is clear is that gorgonians, and octocorals generally, lack reliable phylogenetic hypotheses and much work is yet to be completed (Sánchez et al. 2003a). A combination of specific markers still may provide sufficient resolution to make inferences on both phylogenetic and evolutionary 
principles, even though not being as informative as sequencing the entire genome. Thus, for the purpose of this review Bayer's somewhat tenuous three-group (suborders Holaxonia and Calcaxonia, and Scleraxonian group) system, currently utilised by most octocoral taxonomists (e.g., Fabricius \& Aldersade 2001, Daly et al. 2007; Figure $1.3 \&$ 4), will be referred to with reference to further studies where appropriate.

\subsection{MORPHOLOGY}

Gorgonian morphological variability, whether environmentally plastic, genetically derived or the product of genotype-by-environment interaction(s), display a variety of forms within and between habitats. Colony morphology can be arborescent, flabellate/fan-shaped, spiraled, planar, pinnate, tangled/untangled bushes, lyrate, candelabra, reticulate, encrusting, lobular, or a combination of such forms (Bayer et al. 1983, Fabricius \& Alderslade 2001). Colonies display nested modularity from branched (or unbranched): e.g., reticulate, alternate, pinnate, irregular, dichotomous, monopodal, fistulate, or a combination; polyps (modules sensu stricto) typically retracted or contracted (Figure 1.2a - e); and supra-modular traits e.g., intercalice distance, calice diameter and branch length (Sánchez et al. 2007, Prada et al. 2008). There is considerable variability within such traits, apparently independent of common ancestry, with no complete understanding of developmental or evolutionary processes (Sánchez 2004, France 2007, Sánchez et al. 2007). However, such phenotypic variation among gorgonian individuals is essentially the raw material of natural selection (Sánchez \& Lasker 2003, Pigliucci 2005).

Anthozoans possess developmental genes within the putative Hox1, Hox2, and Hox 9+ gene families giving inference to a rudimentary "Hox code" (Ryan et al. 2007). Possession of such highly conserved gene clusters indicates a greater complexity in form and development for Cnidaria, providing insight into Hox evolution; Hox genes previously considered a bilaterian invention (Ryan et al. 2007). However, knowledge of morphogen gradients regulating cnidarian morphogenesis is poor, yet crucial in developing our understanding on how morphogenesis is controlled by genome-by-environment interactions (Kaandorp \& Kübler 2001). Nonetheless, advances in developmental biology in combination with integrative investigations - in vivo, in vitro and in silico - are beginning to provide valuable insights into the evolution of both developmentally constrained and environmentally dependent gorgonian characters, especially with regard to branching structure (Sánchez et al. 2004, 2007, Brown 2007).

Branching systems are open networks, thus linked between any two points and not closed circuits (Kaandorp \& Kübler 2001). Such networks are present in all complex systems from 
rivers (Horton 1945) to the mammalian nervous system with various indices assigned to branches for morphometric comparisons (reviewed by Kaandorp \& Kübler 2001). Understanding complex gorgonian architecture and developmental processes has lead to numerous comparative morphometeric studies often concluding the potential dependence of growth form on genetic and/or environmental parameters (e.g., Kim et al. 2004, Sánchez et al. 2004, Linares et al. 2008, Prada et al. 2008). Branching gorgonians develop in a sub-apical process, resulting in mother-daughter relationships: the primary (mother) branch producing secondary 'daughter' branches at fixed distances/internodes (Sánchez et al. 2004). The motherdaughter ratio $(c)$ indicates the relationship between colony form and growth (= branching), with colony shape maintained when $(c)$ is constant irrespective of gorgonian species (Sánchez et al. 2004). This pattern is particularly constant in alternate branching taxa and is suggested to indicate physiological developmental constraints or canalization (Sánchez et al. 2004). Furthermore, determinate growth (through self-organized criticality) follows a scaling power law relative to mother branch size frequency distribution. Sánchez (2004) goes on to review differences in colony size and growth patterns whilst $(c)$ is constant, perhaps an example of heterochrony - differences in colony size and shape due to changes in timing or rate of developmental events (Gould 1977). In addition, colony growth will slow asymptotically as the maximum number of mother branches is reached (Sánchez 2004). Interestingly, compensatory growth experiments in the Japanese scleraxonian, Melithaea flabellifera Kükenthal 1908 revealed that optimal size and branch density were determinate by maintaining colony form through irregular and heterogeneous growth (Matsumoto 2004), a compensatory thus determinate pattern also reported in other gorgonians (Sánchez \& Lasker 2003, Kim et al. 2004). Quantification of gorgonian branching networks has further revealed an emergent level of module integration at the colony level (internode distance and branch length; Sánchez \& Lasker 2003). Patterns of morphological trait integration are independent of polyp iteration; a few changes at the polyp level having no significant affect on colony architecture (Sánchez \& Lasker 2003, Sánchez 2004). Nevertheless, character trait inter-dependence may be the product of heritable pleiotropy (multiple phenotypic traits due to a single gene), linkage disequilibrium, or concerted evolution operating on traits for a specific function (Sánchez \& Lasker 2003). Moreover, convergent evolution (homoplasy) in gorgonian colony architectures, first proposed by Bayer (1953), is both phylogenetically corroborated (Sánchez et al. 2003b, Wirshing et al. 2005, Aguilar \& Sánchez 2007, Cairns \& Bayer 2009) and frequently observed between closely related species (Sánchez 2004, Watling et al. 2012). 
Heterogeneity in form exists due to the feedback between growth, and micro- and macrophysical environments (Kaandorp \& Kübler 2001). Thus, branching, growth and form are continuously undergoing physiological adjustments relative to environmental change (Velimo 1975, Matsumoto 2004, Roark et al. 2006). However, gorgonians have a slow growth rate (Grigg 1974, Noé \& Dullo 2006, Tracey et al. 2007), such that investigations of eco-phenotypic and genetic effects using reciprocal transplants principally focus on microstructure variation (sclerite; e.g., West et al. 1993, West 1997) and specific genetic markers (Prada et al. 2008, Gutiérrez-Rodríguez et al. 2009). Furthermore, gorgonian transplant experiments at opposite ends of an environmental gradient such as depth, frequently revealed phenotypic plasticity, thus environmentally induced traits (Bayer 1961, Brazeau et al. 1991, West et al. 1993, West 1997, Kim \& Lasker 1997, Kim et al. 2004, Skoufas 2006). Such phenotypic expression aligned with differences in genetic markers, but these markers (e.g. mshl, ITS - caveats discussed above) are not specific for the phenotypic trait(s) observed and are limited in their ability to investigate environmental challenge responses. Interestingly, detection of branching initiation in the gorgonian Pseudopterogorgia [now Antillogorgia] elisabethae Bayer 1961 using the Hox marker anthox revealed differences in gene expression within and between branch locations, yet failed to give sufficient resolution as a marker for branch initiation (Brown 2007). Thus, much work on the expression of genes specific to phenotypic plasticity has yet to be conducted whether from the genome or of epigenomic origin.

The interplay between gorgonian developmental gene expression and resource supply is unknown, however use of simulation models and morphometric analyses may infer resource allocation structure and surplus, further triggering phenotypic plasticity events. Colony architecture determines its own morphological trajectory in marine hydroids. Sheet and runnerlike morphologies influence polyp pumping activity that in turn reduces or increases gastrovascular flow rate and relative cellular oxidation states in developing hydroids (Blackstone \& Buss 1993). The resulting internal tensions trigger developmental gene expression (e.g., Cnox). Whether such colonial expansion thresholds exist in gorgonians remains to be seen, however, determinate growth appears to be 1) evident (e.g. Matsumoto 2004, Sánchez \& Lasker 2003, Sánchez et al. 2004, Lasker et al. 2008), and 2) most likely influenced during early life stages (Cossins et al. 2006).

\subsection{ECOLOGY}

Ecologically diverse, gorgonians are important components of marine habitats from the deep sea to the tropical intertidal (Fabricius \& Alderslade 2001, McFadden et al. 2006). Gorgonian 
abundance, diversity and distribution depend upon environmental factors such as substrate type, light, temperature, sedimentation, current regime and flow rates (Garrabou et al. 2001), which have been shown to induce intra- and interspecific morphological variability both within and between individual colonies (van Oppen et al. 2005). Yet little is known of the ecology, biology and variable phenotypic responses of gorgonian taxa relative to environmental parameters, particularly in both the deep sea (Parrish 2007) and the Indo-Pacific (van Oppen et al. 2005).

Gorgonian ecology often reflects reproductive strategies and/or changes along environmental gradients relative to individual species tolerances (Fabricius \& Alderslade 2001). Gorgonians reproduce both sexually and asexually, with a variety of strategies having differing effects on population growth (Lasker 1990, 1996, 2006, Lasker et al. 1988 1996), even including parthenogenesis (Brazeau \& Lasker 1998). Most research has been conducted on Caribbean and Mediterranean taxa, however, the majority being internal brooders (sperm cast, sensu Pemberton et al. 2003) with short pelagic larval duration before settlement. Nevertheless, staggered or neap tide spawning events, planktonic larval displacement by water currents, and chemotaxis through conspecific or coralline algal exudates (Fabricius \& Alderslade 2001) may further influence local distribution, abundance and survival. In addition, gorgonians are subject to considerable endemism (Grasshoff \& Bargibant 2001, Piccianno \& Ferrier-Pagès 2007) with just a single shallow water gorgonian (Acabaria bicolor Nutting 1908) in the Hawaiian Islands and American Samoa (Fenner pers. comm., 2010). Nonetheless, shallow water gorgonians are highly abundant and predominantly zooxanthellate in the Caribbean compared to the Indo-Pacific, by far the most diverse, yet little researched taxa (Grasshoff \& Bargibant 2001).

Most gorgonian species are restricted to relatively small areas, such as islands (e.g., Aldabra, Bayer 1996; New Caledonia, Grasshoff \& Bargibant 2001), with evolutionary processes often constrained by dispersal ability in terms of life history and biogeography. With a low range-size frequency distribution $\left(\sim 4\right.$ million $\mathrm{km}^{2}$ to $>10$ million $\mathrm{km}^{2}$ for low and high range-size dispersing taxa respectively, as a proxy taken from Hughes et al. 2002) and endemism suggested central to the Indo-Pacific, mid-range dispersers may create the potential for the isolation of populations (mid-domain effect: see Colwell \& Lees 2000) or subpopulations thus, vicariance with large dispersing, pandemic taxa having a decreased probability of speciation due to a greater ability to populate wider areas relative to habitat availability (Brown 2014, but see Hughes et al. 2002, Connolly et al. 2003, Colwell et al. 2005, ). However, the mid-domain effect - suggesting an increased overlap of species ranges at the centre of a 'domain' leading to a peak of species richness - appears to be positively correlated with prevailing ocean currents revealing 
high species richness at periferal Indo-Pacific locations in numerous invertebrate groups (Connolly et al. 2003, Budd \& Pandolfi 2010). Furthermore, increasing evidence reveals little correlation with short dispersion time and endemism for many taxonomic groups within the Coral Triangle, lending question to dispersal ability and reproductive mode as effective indicators of biodiversity particularly in the face of habitat availability as present in dense archepelago's such as Indonesia. In other words, if there's always somewhere to settle then dispersal ability is of less importance. Nevertheless, with only little knowledge of gorgonian species distribution, developmental strategies and taxonomy per se, it is not clear how such hypotheses extend to this group, diminishing their potential utility as a conservation indicator group for effective management strategies.

Gorgonians show considerable phenotypic plasticity likely as an important factor contributing to their broad distribution as a group, which may further lead to genetic accommodation and/or assimilation and divergence (West-Eberhard 2003, 2005). However, gorgonian responses to environmental parameters vary across taxa, with zooxanthellate taxa absent in highly polluted areas, and tolerant azooxanthellate taxa often having a high susceptibility to fungal infections, colonization by fouling organisms, and a high partial mortality (Fabricius \& Alderslade 2001). Complex habitats provide more vertical relief, colonizable area, and greater microhabitat variability than soft benthic substrata (Etnoyer et al. 2010). Yet even in the presence of suitable substratum, most gorgonians are absent in areas of high turbidity due to the physical impairment of settlement, feeding, reproduction and growth. Habitats characterised by low wave action, high turbidity and sedimentation rates, favour encrusting Briareum spp. (Fabricius \& Alderslade 2001), likely due to morphological and behavioural pre-adaptations such as phenotypic and photoacclimatory plasticity, colony dynamics, polyp density and size, reproductive strategy and recruitment survival (Stafford-Smith 1993, Anthony 2000). However, turbid habitats are marginal for zooxanthellate gorgonians, with no evidence of hard coral community replacement. Unsurprisingly, zooxanthellate gorgonian taxa follow similar depth ranges to scleractinia, however their relative reliance on endosymbiont photosynthetic carbon appears to be species specific (Sorokin 1991). Furthermore, high water motion and localized upwelling provide elevated nutrients for primary productivity and enhanced food availability (Jokiel 1978, Reed 1983, Sebens 1984). Arborescent branching gorgonians orientate themselves perpendicular to the predominant water current in order to maximize food capture (Grigg 1972, Fabricius \& Alderslade 2001). Pristine, high hydrodynamic conditions facilitate the largest colonies, but can also limit colony size (Linares et al. 2008) and enhance relative gorgonian diversity. Such patterns may be attributed to intermediate disturbance levels, maintaining relative species 
diversity within a reef community (Connell 1978, Ostrander et al. 2000). Moreover, scaling effects may also lead to determinate growth and size whereby colony size reaches its functional capacity. Taken together, sedimentation, light and water flow appear major factors controlling local gorgonian populations (Meesters et al 2001, Linares et al. 2008).

Gorgonians are ecologically diverse, long-lived, slow growing engineering species (species which "modify, maintain and create habitats." Jones et al. 1994) with growth rates as little as 14 $\mu \mathrm{m} \mathrm{yr}^{-1}$ (Roark et al. 2006), making them effective bioarchives (Risk et al. 2007, Williams \& Grottoli 2010) yet vulnerable to disturbance, which can dramatically affect whole communities (Linares et al. 2008). Deep sea and Mediterranean gorgonian reefs are particularly vulnerable to the commercial harvesting of precious corals (Corallium spp.; Grigg 2001, Santangelo et al. 1993) and bottom trawling (Watling \& Norse 1998, Hall-Spencer et al. 2002). Furthermore, susceptibility to bleaching events and disease outbreaks has increased dramatically, having profound effects on gorgonian taxa in tropical (Smith et al. 1996, Geiser et al. 1998) and temperate regions (Cerrano et al. 2000, Garrabou et al. 2001, Hall-Spencer et al. 2007), likely due to global climate change. Gorgonians are therefore conservation indicator taxa, providing both habitat and refugia for numerous organisms including commercially important juvenile and adult fish species (Hall-Spencer et al. 2002). Understanding patterns of gorgonian ecology, physiology and morphological variation through cross-disciplinary approaches will be increasingly important in management and remedial conservation efforts.

Due to their longevity and architectural diversity numerous marine organisms are associated with gorgonians, commonly exhibiting novel phenotypic adaptations. Most notably are morphological mimics such as the charismatic pigmy seahorse taxa e.g., Hippocampus denise Lourie \& Randall 2003, facultative to various Scleraxonian and Holaxonian hosts (Lourie \& Randall 2003). Unlike seahorses however, associates such as the gastropod Cyphoma gibbosum Linnaeus 1758 predate upon the polyps of their host, now the principle predator of Atlantic shallow water gorgonians as a result of predator release from over fishing (Birkepile \& Hay 2007). Furthermore, C. gibbosum has recently been traced as a likely vector of the fungus Aspergillus sydowii (Bainier \& Sartory) Thom \& Church 1926, which decimated populations of the Caribbean gorgonian Gorgonia spp. (Smith \& Weil 2004). However, compared to hermatypic (hard) corals gorgonians have few specialist predators (Puglisis et al. 2000), probably the result of secondary metabolite production in response to competition (Kim \& Lasker 1997, Van Oppen et al. 2005), predation and changes in environmental stimuli (West et al. 1993, Hoover et al. 2008). Interestingly, transcriptome analyses have revealed an induced 
chemical response to predation stress by Chaetodon spp. on the soft coral Sinularia polydactyla Eherenberg 1834 (Hoover et al. 2008). Such metabolic responses are thought to increase in both variability and abundance at lower latitudes likely due to increased predation and competition (Puglisi et al. 2000).

Irrespective of their battery of chemical defenses, and alloimmunity (see Salter-Cid \& Bigger 1991), certain gorgonian taxa are particularly susceptible to fouling, often as a consequence of mechanical damage, pollution or predation reducing reproductive output, further leading to a colonization cascade of opportunistic fouling organisms commonly resulting in host mortality (Gerhart 1990, Weinbauer \& Velimirov 1996). Fouling extent has been attributed to increases in temperature or light (Drohan et al. 2005), as is also the case with disease (Cerrano et al. 2000, Harvell et al. 2001). Nevertheless, associate organisms have also been shown to remove sediment and consume boring and fouling larvae (Goh et al. 1999). Such symbioses are thought to be advantageous, gorgonians having reduced mucus secretary cells compared to other Cnidaria (Fabricius \& Alderslade 2001).

\subsection{TROPHIC ECOLOGY}

Thus far, it is evident that gorgonians are modular organisms, with determinate colony growth, form and size (Lasker et al. 2003) due to the iterative addition of polyps and branches, and within colony canalisation providing effective resource allocation structure (Sánchez \& Lasker 2003). Furthermore, colony form can depend on feeding strategy and the same genotype can show different allocation patterns in different environments, consistent with the 'partitioning' hypothesis (Poorter \& Nagel 2000, Weiner 2004).

Growth form and resource allocation also change to counter the effects of environmental factors such as sedimentation (Riegal \& Branch 1996) and depth (West 1997). Moreover, sedimentation has profound effects on coral metabolism by decreasing photosynthetic productivity in zooxanthellate gorgonian taxa, and increasing respiration (carbon-loss) by $95-100 \%$ through increased mucus production (Riegl \& Branch 1995). The long-term effects of such expenditure on gorgonians are unknown, with virtually nothing known about gorgonian coral symbioses in the Indo-Pacific.

Most gorgonians are colonial suspension feeding heterotrophs, predominantly capturing suspended particulate organic matter (POM; Tsounis et al. 2005, Picciano \& Ferrier-Pagès 2007), as well as dissolved organic matter (DOM) and zooplankton (Fabricius \& Klumpp 1995, 
Fabricius \& Alderslade 2001). Certain shallow water taxa particularly in the Caribbean have a moderate dependence on phototrophy, harbouring symbiotic zooxanthellae within the gastrodermal tissue (Fabricius \& Alderslade 2001), with a concomitant plasticity in growth form relative to the environment (Kaandorp \& Kübler 2001, Prada et al. 2008). Phenotypic variability relative to their surrounding habitat such as colouration (Sánchez et al. 2007), branching dynamics (e.g., Matsumoto 2004, Sánchez 2004), colony surface area, polyp density and intercalice distance (West et al. 1993, Prada et al. 2008) have been shown to enhance food capture and efficiency. Most importantly is the extension of polyps - the primary feeding apparatus that can also bear photosynthetic endosymbionts (Symbiodinium Freudenthal 1962) in zooxanthellate taxa - into the water column whereby colony growth and form are interdependent on resource availability. Differential resource allocation patterns (hetero/phototrophic capacity) can vary relative to the environment in the same genotype, with or without morphological change (Sebens 1997, Poorter \& Nagel 2000, Weiner 2004).

Assessing differential resource allocation patterns at the species level particularly in contrasting environments may be informative of the mechanisms of phenotypic variability within and between taxa, and ultimately tractable responses to environmental change. Energy apportionment relative to food acquisition and transfer from the environments and/or symbiont can be measured using stable isotopes of carbon $\left(\delta^{13} \mathrm{C}\right)$ and nitrogen $\left(\delta^{15} \mathrm{~N}\right)$. However, gorgonian research has focused on the relative isotopic signatures of the calcareous axis and skeletal elements (sclerites) usually as bioarchives (sensu Williams \& Grottoli 2010, Risk et al. 2002). For example, azooxanthellate gorgonian skeletal $\delta^{13} \mathrm{C}$ increased as nitrogen $\delta^{15} \mathrm{~N}$ decreased with increasing depth, further correlating with suspended POM values (Williams \& Grottoli 2010). Feeding experiments on Corallium rubrum Linnaeus 1758 also revealed a preference for autotrophic flagellates that increased with temperature (Picciano \& Ferrier-Pagès 2007). Yet experiments on numerous shallow water taxa within the Great Barrier Reef revealed that the heterotrophic food source was species-specific irrespective of zooxanthellate status (Sorokin 1991). Nevertheless, until recently (Baker et al. 2011) nothing was known of the isotopic ratios of gorgonian soft tissues, primarily due to the unsuccessful separation of sclerites giving erroneous $\delta^{13} \mathrm{C}$ signatures (Grottoli pers. comms. 2010). Interestingly Baker et al. (2011) revealed using Caribbean zooxanthellate gorgonians, that light primarily affected $\delta^{15} \mathrm{~N}$ fractionation even though the values were minimal; this still has bearing on differentiating the effects of pollution on coral reef taxa. Comparative analyses of soft tissue and endosymbiont isotopic ratios in alignment with the surrounding environment would further elucidate the relationship between host, symbiont and their intrinsic and extrinsic energy transfer role(s) in 
benthic - pelagic coupling. Moreover, exploring how endosymbiont (i.e., Symbiodinium) types influence the physiological performance of the holobiont to environmental change would greatly enhance our understanding of what drives host-symbiont associations.

The photosynthetic dinoflagellates within the genus Symbiodinium associate with numerous coral reef invertebrates, most notably the hermatypic (reef building) Scleractinian corals. Diversity within the genus Symbiodinium is under continual investigation, but is hampered by a lack of morphological distinction between sub-generic clades (A-I; Pochon \& Gates 2010) with differences identified at the genetic level (e.g., LaJeunesse 2002, Pochon et al. 2006, 2012). Technological advances continually reveal novel and cryptic variation (e.g., Silverstein et al. 2012) increasingly associated with photophysiological tolerance (Jones et al. 2008, LaJeunesse et al. 2008, Hennige et al. 2009, but see Abrego et al. 2008) and the complex interplay between host and symbiont. Zooxanthellate gorgonians are major reef components on Caribbean reefs, but only a few taxa are present in the Indo-Pacific. Most show symbiont specificity (Goulet et al. 2008) with Briareum Gray 1859 and Isis hippuris Linnaeus 1758 known to harbour the putatively stress tolerant clade D Symbiodinium (van Oppen et al. 2005). Interestingly, both transplant and laboratory experiments on the gorgonian Briareum sp. reveal symbiont 'switching' in response to environmental parameters in the Caribbean (Lewis \& Coffroth 2004); a proposed adaptive response borne from the nonselective Symbiodinium acquisition by juvenile hosts (Coffroth et al. 2001). Thus, specific Symbiodinium spp. in different host species across unique physical-environmental conditions may be linked to abiotic regime. Furthermore, algal clade selection by either symbiont 'switching' (exogenously) or symbionts 'shuffling' within a host coral colony is controversial (Baker 2003, Goulet 2006, Apprill \& Gates 2009), as either mechanism assumes that the coral species can host multiple algal genotypes, sequentially or simultaneously. Increasing evidence suggests such a phenomenon (Baker et al. 2004) as a mechanism of survival over the numerous climate and sea level fluctuations, with relatively little extinction in scleractinian corals alone over the last 220 MY (Veron, 1995). Yet many species host only a single Symbiodinium clade regardless of environmental conditions or transplantation experiments e.g., Fungia [now Lobactis] scutaria Lamarck 1801 retained its original zooxanthellae type $(\mathrm{C} 1 \mathrm{~b})$ for 35 yrs after transplantation from the Indo-Pacific to the Caribbean (LaJeunesse et al. 2005). Mechanisms of endosymbiont acquisition and diversity however, remain to be elucidated in Indo-Pacific zooxanthellate gorgonians, particularly within the Coral Triangle. 
Intriguingly, molecular and histological evidence revealed two previously described azooxanthellate gorgonian species, Junceella fragilis Ridley 1884 in the Philippines and Euplexaura nuttingi Kükenthal 1919 on the Great Barrier Reef, as possessing clade G of the symbiotic zooxanthellae Symbiodinium (van Oppen et al. 2005, Williams et al. 2010). Individuals were found in shallow turbid waters and lacked host pigmentation in contrast to their brightly coloured deeper azooxanthellate counterparts. Such evidence may represent differential phenotypic expression of a genotype under varying environmental conditions (West et al. 1993), in addition to raising questions on the obligate nature of Symbiodinium with such taxa. The significance of such a discovery, and if enhanced fitness through mixotrophy in turbid environments is adaptive plasticity or plasticity as an adaptation, remains to be elucidated. Yet a low reliance on photosynthetic gain increases the likelihood of survival under high temperature and/or irradiance stress. Interestingly, the cnidarian-algal symbiosis has been shown to be maintained by altering the expression of existing genes involved in vital cellular processes, and is thus not due to 'symbioses-specific' genes (Rodriguez-Lanetty et al. 2006, 2008).

\subsection{PHENOTYPIC PLASTICITY}

Central to evolutionary theory is the historical connectivity of all life within and between the environment, and the ability for biological change irrespective of scale. Whether at the population, species, individual, phenotypic trait or molecular level, evolution is considered inherent - the decent of biological variation through natural selection (Darwin 1859) or the nonadaptive influences of genetic drift (Hurst 2009). Such biological variation in response to environmental heterogeneity reinforces survival and reproductive success, particularly in sessile taxa when subject to novel environments. Variability at the phenotypic level (morphological, behavioural, and/or physiological) is conditionally expressed relative to environmental cue(s), within a single generation. In other words, phenotypic plasticity depicts multiple phenotypes from a single genotype in response to environmental variation (Pfenning et al. 2010). Thus, such phenotypic variability occurs within the lifespan of an individual as a consequence of high plasticity capacity or the release of cryptic genetic variability through environmental stress (evolutionary capacitance breakdown; Rice 2008). With such variation, particularly at the phenotypic level, how can one delimit a species and, therefore, differentiate between plasticity capacity and divergent taxa? Moreover, does phenotypic plasticity provide the foundation for novel species through the selection of complex traits that enhance fitness and overall reproductive success, or does it simply obscure selection and species boundaries? Controversy continues to exist with regards the roll of phenotypic plasticity in diversification and speciation (Pfenning et al. 2010), but first, how does it arise and how, if at all, can it be assessed? 
Phenotypic plasticity in response to environmental heterogeneity may be adaptive or genetically derived (Gotthard \& Nylin 1995, Hoogenboom et al. 2008). Plasticity itself can be the result of adaptation, with selection acting on a trait or an organism's ability to be plastic (adaptive plasticity). Intrinsic (development, life history, physiological, or genetic) and extrinsic factors (substrate, light, temperature, sedimentation, competition, predation, and hydrodynamics), alone or in concert, control phenotypic plasticity, the interaction of which can be visualized through reaction norms (Figure 1.5; Gotthard \& Nylin 1995). Reaction norms, a set of phenotypic expressions of a single genotype over an environmental range/gradient (Stearns et al. 1991), visualize the plasticity capacity of a genotype. Through reciprocal transplantation of contrasting phenotypes between opposing environments over an appropriate timeframe, reaction norms can reveal inducible plastic or fixed traits, with concomittent fitness through survivability (Prada \& Hellberg 2013). Phenotypic trait variance (Vp) in the singular or plural may be due to genetic $\left(V_{G}\right)$, environmental $\left(V_{E}\right)$ or interactive effects $\left(V_{G x E}\right)$ where genotypes differentially respond to their environment, and can be expressed as:

$V p=V_{G}+V_{E}+V_{G x E}+V_{\text {error }}$

where $V_{\text {error }}$ accounts for developmental, bet-hedging, behavioural or other unaccountable noise. If selection acts on more favourable genotypes this causes a shift in the average environmental effect on a population leading to adaptive plasticity as an adaptation (Figure 1.5d; DeWitt \& Scheiner 2004). Thus the genetic effects of phenotypic variance, or not as the case may be (i.e., canalisation), underpin plasticity capacity and can be depicted as:

$V_{G}=V_{G, A}+V_{G, D}+V_{G, I}$

where additive $\left(V_{G, A}\right)$, dominant $\left(V_{G, D}\right)$ and epistatic $\left(V_{G, I}\right)$ genetic effects are the result of the relative heritability contribution of allele frequencies to the observed phenotype (Hagemann et al. 1999). Both additive (polygenic) and dominant genes act on specific loci, whereas an epistatic interaction effect is the result of modifier gene(s) at different loci (Johnson 1976, Byers 2008). The quantification of additive variation (average effect of substituting one allele for another) within a given population for e.g., functionally important phenotypic trait(s) reveals the action of incipient divergent selection or genetic drift and ultimately gene flow (Carlon et al. 

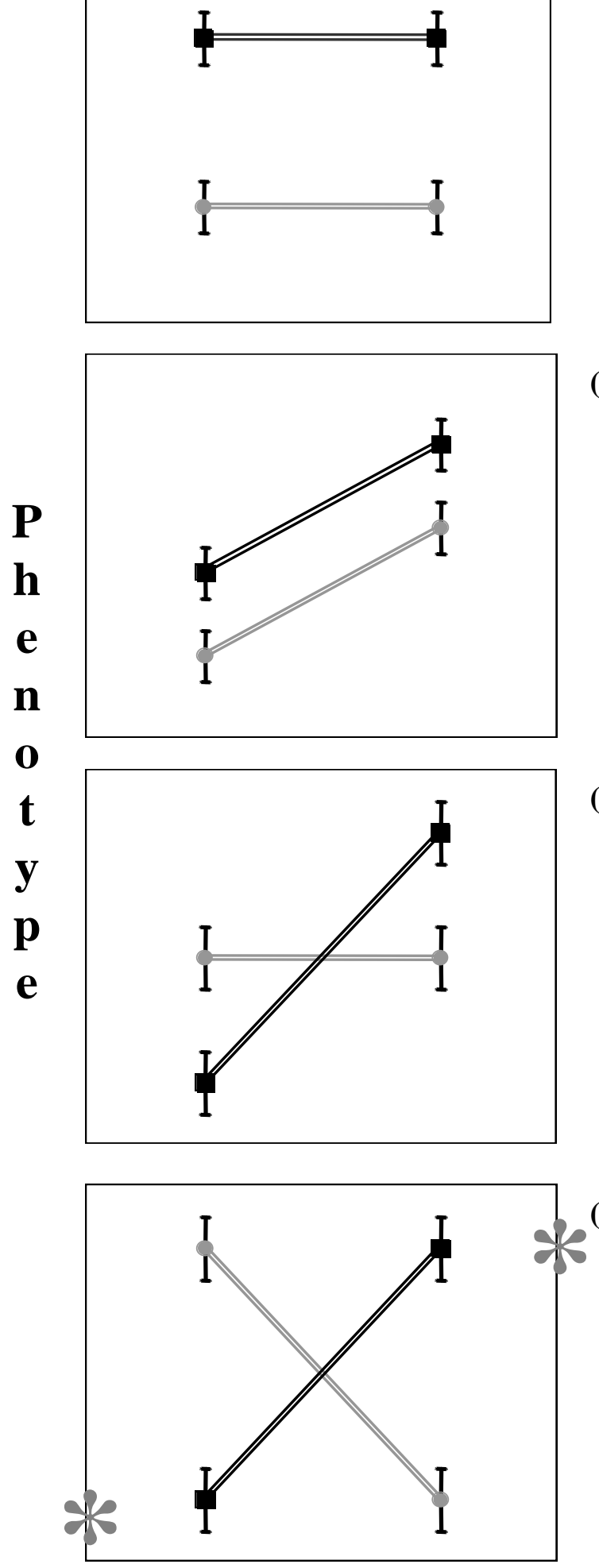

(d) $V_{G x E}$ Genetic and environmental interaction variance: morphotypes have similarly y et opposing reactions to the environment, thus different taxa yet equally as plastic. Adaptive optima (asterisks) indicate that the square morphoty pe/taxon would likely be selected for; adaptive plasticity as an adaptation.

\section{Environment/Time}

Figure 1.5 Reaction norm means of a hypothetical scenario of two morphotypes showing (a) $V_{G}$ genetic variance, (b) $V_{G}$ genetic and $V_{E}$ environmental variance, (c) $V_{E}$ environmental variance and $V_{G x E}$ interaction variance, and (d) $V_{G x E}$ interaction variance (modified from DeWitt \& Scheiner 2004). 
2011, Bird et al. 2012). Furthermore, patterns of covariance between phenotypic traits particularly in contrasting environments, can be indicative of functional trade-offs leading to divergence, however differentiation between adaptive (canalised) and developmentally plastic (epigenetic) influences on the observed phenotype can be inscrutable (DeWitt \& Scheiner 2004). Thus, reaction norms as a product of reciprocal transplant experiements (RTEs) reveal only the source of variation, not the mechanism. Differentiation of plasticity at the mechanistic level would require both field and molecular analyses assessing genetic inheritance and epigenetic developmental effects. Nonetheless, significant population subdivision, particularly with the simultaneous expression of novel phenotypic traits (adaptive radiation; West-Eberhand 2005), is indicative of local adaptation, and thus population and species divergence (Kawecki \& Ebert 2004, Pfenning et al. 2010). As divergent selection acts against intermediate genotypes, the environment is in fact the selective agent acting on plastic phenotypic traits and the ability to be plastic in the first place (Gilbert \& Epel 2009). Thus plasticity can be a diversifying factor, with any number of possible trait combinations that may lead to the production of a novel phenotype, enhancing individual and/or population fitness in a particular environment (Santelices 1999, Magwene 2001b). Intrinsic and extrinsic factors will, therefore, shape species range size (Hughes et al. 2002), essentially reflecting processes of speciation, extinction, resilience to environmental change and overall species diversity and ecosystem functioning.

Fitness enhancement through an individual's (genotype) ability to adapt in heterogeneous environments is particularly important in sessile marine organisms, due to their inability to relocate to another environment. Selection, therefore, acts on plasticity capacity, with modular colonial invertebrates being arguably the most pliable to their physical environment (Kaandorp \& Kübler 2001). Modular and colony growth and form are intrinsically linked to optimise resource acquisition, reproduction and minimize metabolic costs. Fundamental however, is the understanding of interactions between, and relative contributions of, the genome and physical environment to morphogenesis, which in most cases is unknown (Gutiérrez-Rodríguez et al. 2009).

An organism's response to its environment involves numerous biological mechanisms with population dynamics closely tied to resource allocation success, thus environmental regime (Weiner 2004). Multivariate phenotypic traits provide the observed phenotype at any one time which may be a consequence of plasticity capacity, strict adaptation thus canalization (genotypic), a combination of the two on various traits, or unique hybridization through repetitive introgression (Ladner \& Palumbi 2012). How to differentiate such phenotypes as 
actual and informative species in the context of biodiversity assessment for conservation management, can be an onerous task. Arguably one of the greatest challenges is species determination on shallow reefs within the Coral Triangle, exhibiting fitness enhancement through intense competition and the fixation of mutation through free ionization energy (Nei 2007) at low latitudes ('evolutionary rate hypothesis'; Rohde 1992, but see Weir \& Schluter 2007). The Coral Triangle is a recognized "biodiversity hotspot", characterized by high species numbers and endemism, extending from the Philippines to the Solomon Islands (Carpenter \& Springer 2005, Hoeksema 2007, Veron et al. 2009, Gaither \& Rocha 2013). The Indonesian archipelago, within the Coral Triangle, is likely one of the greatest areas of marine biodiversity due to geological age, highest number of islands per unit of geographic area, and ecoclimatic stability lending greater time for evolutionary processes. The origins of such biodiversity are, however, controversial (Gaston et al. 1998, Chown \& Gaston 2000, Bowen et al. 2013) and likely taxon-specific, mechanistic convergence relative to individual fitness response, with latitude itself being just a correlate for the mechanism(s) concerned. Nevertheless, such immense biodiversity is being destroyed, in an area still relatively unexplored, resulting in rudimentary biodiversity assessments and theoretical postulation (e.g., Hughes et al. 2002, Carpenter \& Springer 2005). Comparative research across contrasting (healthy versus exploited) environments may reveal evolutionary mechanisms from plasticity to divergence through evolutionary capacitance in high energy, biodiverse, yet exploited habitats; therefore elevating descriptive biodiversity assessments to realistic conservation measures, from necessity to emergency in the face of anthropogenically induced destruction.

\subsection{RESEARCH PERSPECTIVE}

Understanding patterns of gorgonian ecology, physiology and morphological variation through cross-disciplinary approaches is essential for characterizing species, communities and population resilience to environmental change - increasingly important in management and remedial conservation efforts. Gorgonians show considerable trait variability, independent of common ancestry with such phenotypic plasticity likely to be an important factor contributing to their broad distribution. Furthermore, fitness can be an individual's ability to change. Whether such plasticity in gorgonian taxa is ecologically driven, genetically derived or a combination of the two is continuously explored through genetic, ecological, physiological, and recent advances in genomic research. Gorgonians as modular colonial organisms therefore, provide dynamic models in which to study evolutionary mechanisms as a consequence of their environment. Nonetheless, plasticity is an emergent property of the genotype, therefore it is susceptible to natural selection (Pigliucci 2005). 
Clearly, gorgonian corals represent a highly diverse model group, yet it is surprising that the most diverse of all gorgonian taxa exist within an area that is least understood, particularly the Indonesian archipelago (van Ofwegen 2004), arguably the most biodiverse region of the coral triangle (Carpenter \& Springer 2005, Hoeksema 2007, Veron et al. 2009, 2011). What species are present and how do such seemingly delicate organisms respond to their environment, especially to increasing threats through anthropogenic encroachment? What species exhibit marked biological success, opportunistic in the face of environmental change, and is such success expressed through morphological variability? Would such morphological variability both within and between taxa lead to resource allocation change, mitigating the effects of environmental change? If so, what are the relative contributions of genetic variation (plasticity capacity as an adaptation) and eco-phenotypic plasticity (e.g., acclimation) to the observed phenotypic variation? These questions are addressed utilising cross-disciplinary approaches on gorgonian taxa within the Wakatobi Marine National Park (WMNP), SE Sulawesi, Indonesia the second largest national park in the country. Investigations are set to assess (1) shallow-water gorgonian diversity, and (2) subsequently infer plasticity or adaptive evolution in observed gorgonian morphotypes across environmental clines of both natural and anthropogenic origin.

\subsection{AIMS \& OBJECTIVES}

With the purpose of highlighting the importance of gorgonian corals in marine ecosystems, specifically within the Coral Triangle, this research aims to evaluate gorgonian responses to environmental change predominantly as a consequence of anthropogenic disturbance. Ultimately, through underlying evolutionary principles, a sequential investigation of gorgonian ecology, taxonomy, and phenotypic dynamics, this study aims to increase both our understanding and awareness of this group as key indicators of reef health, influential in environmental impact management strategies.

To address how gorgonian corals respond to environmental change within the WMNP, SE Sulawesi, Indonesia, this research, in order of each chapter, aims to:

1. Characterise gorgonian diversity, abundance and distribution patterns across gradients of habitat quality within the WMNP.

2. Identify biologically successful gorgonian species across such environmental clines.

3. Further identify predictor environmental variable(s) inferred to influence the ecological 
structure of gorgonian assemblages.

The first three objectives are achieved in Chapter 2 through a stratified ecological survey measuring corresponding environmental variables within each site. It is hypothesized that gorgonian corals can tolerate only clear, moderate to fast flowing hydrodynamic reef environments, and are therefore markedly reduced or absent in opposing habitats.

4. Quantify observed morphological variability in the zooxanthellate gorgonian Isis hippuris Linnaeus 1758 across environmental gradients on reefs within the WMNP.

5. Determine whether such I. hippuris morphotypes are both phenotypically and genetically partitioned across contrasting reef environments.

6. Further ascertain if the observed I. hippuris morphotypes represent previously described species, new species or a single species with highly variant, integrated phenotypic traits.

These three objectives are addressed in Chapter 3 firstly, assessing the taxonomic history of $I$. hippuris in the context of the two distinct morphotypes found within the WMNP (Chapter 2); and secondly by using both anatomical and molecular morphometrics between such morphotypes on the contrasting reefs. It is hypothesized that such phenotypic variability is merely plasticity as an adaptation in a single species across environmental clines. Further insight is also given into the phylogenetic position of I. hippuris within the sub-Class Octocorallia.

7. Determine if I. hippuris morphotypes across environmental gradients are environmentally induced (plastic) or genetically derived (canalized/adaptation).

8. Assess differential physiological responses of the I. hippuris holobiont to environmental change.

9. Investigate host-algal endosymbiont specificity between morphotypes across and as a consequence of environmental change.

10. Determine integrated phenotypic traits which interact to delimit I. hippuris morphotypes suggesting mechanisms of divergence through phenotypic trait integration in response to environmental perturbation. 
Objectives 7 to 10 are addressed in Chapter 4 using a combination of multi-trait (morphology, endosymbiont type, and physiological components) and environmental measurements from a one-year reciprocal transplant experiment between sites of contrasting reef health at comparable optical depths. It is hypothesized that light availability is a primary vector (causal) of I. hippuris morphotypes further driving integration among phenotypic traits. The quantum efficiency of the I. hippuris holobiont through the functional integration of optical traits was therefore assessed through a reciprocal transplant experiment (RTE) for evidence for the onset of light-induced directional selection or plasticity capacity.

11. Summarise both the importance and status of Indonesian gorgonian octocorals as a consequence of this research.

This final objective aims to juxtapose this study's findings with existing and proposed research, highlighting integral knowledge gaps in the second-most common coral reef component in a region of high yet insufficiently researched biodiversity. Furthermore, the phylogenetic implications of this research are considered in the context of the family Isididae and the significance of certain phenotypic traits, in particular the central axis, as objects of selection; inherent or convergent?

All chapters are to be, or are in the process of submission for publication with the exception of chapters 1 and 5 which will be merged for publication as a single review. 


\title{
CHAPTER 2: ENVIRONMENTAL GRADIENTS STRUCTURE GORGONIAN ECOLOGY ON CORAL REEFS IN SE SULAWESI, INDONESIA.
}

\begin{abstract}
Indonesian coral reefs are the epicentre of marine biodiversity, yet are under rapid anthropogenically-induced decline. Therefore, the necessity for ecological monitoring of high diversity taxa facilitating effective management and conservation is paramount. This study presents a unique and comprehensive survey of shallow-water (0-15 $\mathrm{m})$ gorgonian assemblage composition and structure across a gradient of habitat quality within the Wakatobi Marine National Park (WMNP), SE Sulawesi, Indonesia. A total of 197 species and morphotypes from 41 genera and 12 families within the Calcaxonian, Holaxonian and Scleraxonian groups, are reported with current estimates of 21 new species and 28 new species records for the region. Results from this extensive survey confirm high local gorgonian abundance, diversity and species richness in the absence of anthropogenic influence and increasing with depth. Notably, morphological variants of the zooxanthellate species Isis hippuris Linnaeus 1758 and Briareum Blainville 1830 drive site and habitat assemblage differences across environmental gradients. Azooxanthellate taxa particularly within the Plexauridae drive species richness and diversity with depth. However, collinearities among 14 predictor variables explained only $30 \%$ of gorgonian assemblage structure highlighting benthic characteristics, water flow and natural light as primary ecological drivers. Thus non-independence between zooxanthellate $(\mathrm{S}=8, \mathrm{n}=1900)$ and azooxanthellate $(\mathrm{S}=189, \mathrm{n}=1517)$ taxa partitioned distinct gorgonian communities into two trophic groups: autotrophs and heterotrophs respectively, with contrasting diversity and abundance patterns within and between study sites. Such trophic group partitioning and habitat specific morphotypes suggest resource allocation structure representing both alternate feeding strategies and acclimatory phenotypic responses to anthropogenic impacts on coral reefs. This study strongly supports the WMNP as an area of high regional gorgonian abundance and diversity with results undoubtedly propagating conservation and research benefits beyond those presented here.
\end{abstract}

Key words: Gorgonian corals · Indonesia $\cdot$ Ecology $\cdot$ Environmental Gradient $\cdot$ Coral Reefs 


\subsection{INTRODUCTION}

The Indonesian Archipelago is central to marine biodiversity, likely consequential of geological and oceanographic processes influencing species diversification and persistence (Carpenter et al. 2011) at local and regional scales. Eastern Indonesian reefs are particularly diverse, with low climatic variability and strong seasonal upwellings, yet ecological assessments are sparse (Edinger et al. 2000, Tomascik et al. 2004). Increases in human population growth, continual marine resource exploitation through coral mining, cyanide, dynamite, and subsistence fisheries mean such biodiverse ecosystems are being destroyed before their components are discovered. Therefore, comparative assessment of coral reef communities relative to their environment, including the increasing assortment of anthropogenic influences, provides an essential resource for conservation management.

Gorgonian corals (Cnidaria: Anthozoa: Octocorallia) are conspicuous, diverse and often dominant components of benthic marine environments, notably tropical shallow reefs, deep-sea, and mesophotic habitats (Wirshing et al. 2005, Cerrano et al. 2010, McFadden et al. 2010a). Numerous gorgonians are conservation 'flagship' species (Tinsley 2005, Linares et al. 2008, Cerrano et al. 2010) being ecologically diverse, long-lived engineering taxa that maintain habitat heterogeneity and provide secondary space to other organisms (Buhl-Mortensen \& Mortensen 2004, Buhl-Mortensen et al. 2010). Nevertheless, despite their ecological importance and diversity, the greatest paucity of information on gorgonians continues to exist within the Indonesian Archipelago (Tomascik et al. 2004).

Gorgonian corals are colonial suspension feeders primarily defined by a semi-rigid scleroproteinaceous (gorgonin) axis with varying amounts of calcification (Bayer 1961, Grasshoff 1999, Sánchez et al. 2003). Characteristic of the Octocorallia, their polyps bear eight pinnate tentacles, and eight mesenteries dividing the gastrovascular cavity (Bayer 1961, Berntson 1998). Originally classified under the order Gorgonacea (now taxonomically obsolete), gorgonians currently comprise the somewhat tenuous suborders, Holaxonia and Calcaxonia and the group Scleraxonians within the order Alcyonacea (Bayer 1981). Taxonomic efforts for IndoPacific gorgonians are however, confounded by widespread homoplasy, considerable morphological variability, cryptic and sibling taxa (Knowlton 1993). Classified as "poorly known" (van Ofwegen 2004), shallow water gorgonian taxonomy within Central Indonesia remains in a state of flux requiring resolute cross-disciplinary systematic, molecular and ecological approaches. 
Gorgonian ecology reflects, together or in part, reproductive strategies and changes along environmental gradients relative to individual species tolerances (Fabricius \& Alderslade 2001). Environmental factors such as substrate type, light, temperature, sedimentation, salinity, current regime and flow rate (Garrabou et al. 2001) influence gorgonian demography. Biotic factors further provide local-scale community refinement including competition, predation, symbioses, reproduction, settlement and developmental properties (Sánchez 2004). Such factors have been shown to induce intra- and inter-specific morphological variability (West 1997, 1998, Linares et al. 2008, Prada et al. 2008), habitat selection and colony orientation (Sánchez et al. 2003a). Nevertheless, gorgonians are typically synonymized with areas of low sedimentation and high water flow through strong currents and upwellings (Kinzie 1973, Birkeland 1974, Yoshioka \& Yoshioka 1989, Sanchez et al. 1998), the largest planar arborescent colonies occurring in healthy reef environments (Meesters et al. 2001, Linares et al. 2008). Complex habitats provide more vertical relief, colonizable area, and microhabitat variability than soft benthic substrata (Etnoyer et al. 2010). Yet even in the presence of suitable substratum, most gorgonians have been shown to be absent in areas of high turbidity and sediment load likely due to the physical impairment of settlement, feeding, reproduction and growth (Bayer 1956, Anthony \& Fabricius 2000). In contrast, high turbidity reefs in Singapore, for example, support healthy azooxanthellate gorgonian communities (Goh \& Chou 1994). Reduced irradiance levels may therefore, provide competitive release (Rogers 1990) for azooxanthellate taxa; turbid habitats being marginal for zooxanthellate gorgonians, following similar depth ranges of Scleractinia with no evidence of hard coral community replacement (Fabricius \& Alderslade 2001). Moreover, evidence for negative associations with other benthic space competitors appears absent in other areas (e.g., Yoshioka \& Yoshioka 1989). However, resource partitioning theory predicts habitat specialists (Schoener 1974), as growth form and resource allocation plasticity counter the effects of environmental factors such as sedimentation (Riegl \& Branch 1995) and depth (West 1997).

Colony growth and form are determinate through the iterative addition of polyps, branches, and within colony canalisation providing effective resource allocation structure (Sánchez \& Lasker 2003), all of which depend on feeding strategy. Both azooxanthellate and zooxanthellate gorgonians show eco-phenotypic interactions which strongly correlate with depth (West et al. 1993) and size (Sebens 1982). Yet, whether such trophic division is a consequence of interspecific competition in oligotrophic coral reef systems is uncertain. The question remains, are strong interspecific competitive forces driving shallow water gorgonian ecology within the Indonesian archipelago? 
Gorgonian distribution has been positively correlated with substrate availability and type (Goh \& Chou 1994), localized overlapping of species range sizes (as a function of temperature) and benthic-pelagic coupling (Matsumoto et al. 2007). Yet little is known of both reproductive strategies and relative range sizes for most gorgonian species in the Indo-Pacific, likely having limited dispersal abilities and high endemism (Grasshoff \& Bargibant 2001, Picciano \& FerrierPagès 2007). Moreover, larval recruitment plays a key role in gorgonian community structure (Yoshioka 1996), with staggered or neap tide spawning events (Benayahu \& Loya 1981), planktonic larval displacement by water currents, and chemotaxis through conspecific or coralline algal exudates (Fabricius \& Alderslade 2001) further influencing local distribution, abundance and survival.

Prominent drivers of gorgonian ecology, therefore remain unclear, usually describing regional differences (Singapore, Goh \& Chou 1994; Caribbean, Sanchez et al. 1997; Guam, Paulay et al. 2003; Hong Kong, Fabricius \& McCory 2006; Japan, Matsumoto et al. 2007). However, ecological factors that regulate species diversity, as well as consistency in species nomenclature, are of absolute research and conservation importance especially within the Indonesian archipelago, which is subject to continual overexploitation and habitat loss. Published surveys within Central Indonesia, such as the 'Siboga' (Versluys, 1902, 1906, Nutting 1910a-e, 1911, Stiasny 1937) and 'Snellius' (e.g., Stiasny 1940, Verseveldt 1966) expeditions sampled only deep water and Alcyoniidae taxa respectively, thus largely unrepresentative of shallow water gorgonians on Indonesian reefs. Annual rapid assessment surveys are increasingly conducted by conservation agencies (e.g., WWF, TNC) throughout the Indonesian archipelago, with a view for sustainable conservation management. Such surveys are rudimentary with sparse gorgonian taxonomic resolution. The disparity between gorgonian diversity and ecological assessment within Indonesia is, therefore primarily due to taxonomic uncertainty (Bayer 1981), with concomitant difficulties in field identification (Fabricius \& Alderslade 2001) and dispersal patterns. Yet with continual habitat degradation across Indonesian coral reefs, will certain gorgonian species absorb significant magnitudes of such anthropogenic disturbance through succession and subsequent survival?

Little is known of gorgonian ecology within SE Sulawesi, Indonesia despite their high regional abundance and diversity. The aims of this study therefore, were (1) to characterise gorgonian assemblage composition and structure across a gradient of habitat quality within the WMNP, (2) to similarly assess gorgonian diversity and abundance between reef habitats as a function of 


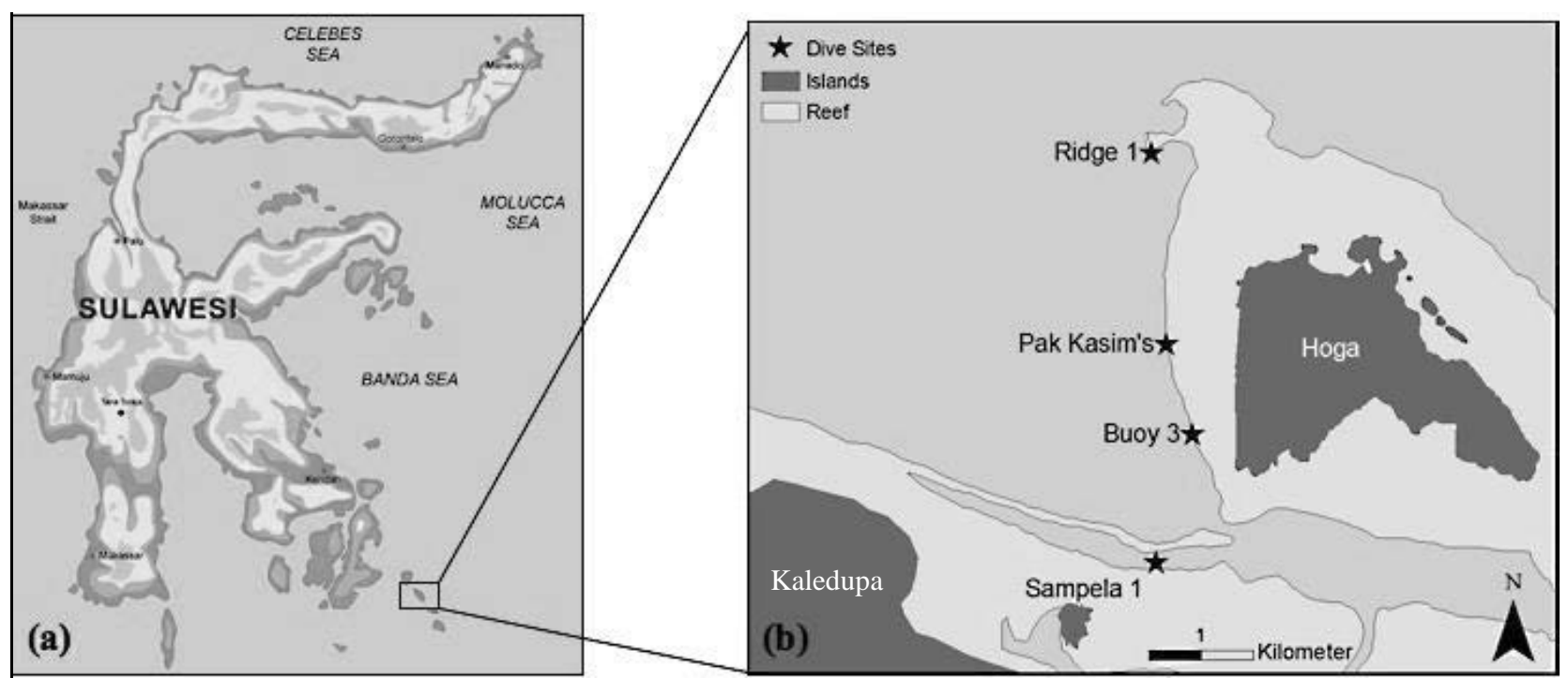

Figure 2.1. (a) Location map of the Wakatobi Marine National Park in S.E. Sulawesi, Indonesia.

(b) Areas of study; Sampela, Buoy 3, Pak Kasim's and Ridge 1 off the islands of Kaledupa and Hoga respectively.

depth within each site, and (3) to identify potential environmental driver(s) of gorgonian assemblage structure.

\subsection{METHODS}

\subsubsection{Study Area}

The Wakatobi Marine National Park (Tukang Besi Islands) is a remote island group of ca. $13,900 \mathrm{~km}^{2}$ in S.E. Sulawesi, Indonesia (Figure 2.1a). Established in 1996, the WMNP is the second largest marine park in Indonesia containing ca. $600 \mathrm{~km}^{2}$ of the most biodiverse coral reefs in the world (Scaps \& Denis 2007), with a low incidence of coral disease $(0.57 \%$ see Haapkylä et al. 2007) and ENSO-induced bleaching events (Crabbe \& Smith, 2003) likely due to local upwelling (Gieskes et al. 1988). Approximately 100,000 people live within the Wakatobi, resulting in extensive subsistence marine resource dependence and destructive commercial fisheries in populated areas. In this study four sites were selected around the islands of Kaledupa (ca. 17,000 people) and Hoga ( $<100$ people, Figure 2.1b) relative to their variability in natural and anthropogenic disturbance. Sampela (impacted), an enclosed lagoon with an outer reef wall ca. $400 \mathrm{~m}$ from a Bajo (sea gypsy) village of ca. 1600 people, is subject to continuous exploitation through coral mining, fishing activities, and high sediment loading due to natural re-suspension, bioturbation through gleaning, and mangrove loss. Furthermore, community wastewater is continually released onto the reef (Haapkylä et al. 2007). Buoy 3 (intermediate I), ca. $500 \mathrm{~m}$ offshore, is a moderately sheltered fringing reef with a shear reef wall containing small cryptic overhang habitats. This site has an extended reef flat, which is subject to perpetual 
'gleaning' of marine invertebrates by local inhabitants, in addition to recovering from coral mining and blast fishing since 2004. Pak Kasim's (intermediate II), ca. $500 \mathrm{~m}$ offshore, is an intermediate topographically complex fringing reef, also subject to coral mining and blast fishing on the reef flat and crest until 2004. Ridge 1 (healthy), ca. $1 \mathrm{~km}$ offshore, is an exposed reef ridge with strong water currents (Figure 2.1b) and upwelling with a small amount of blast fishing on the reef crest in 2004. The reef slope can also be shear, possessing cryptic overhang habitats. All sites have a pronounced reef flat $(<3 \mathrm{~m}$ [Ridge 1 being the exception as an offshore ridge, yet still having a shallow reef plateau ca. $3 \mathrm{~m}$ depth]), reef crest $(3-6 \mathrm{~m})$ and slope (> 6 $\mathrm{m})$ with varying levels of sedimentation draining from the reef flats during spring tides.

\subsubsection{Sample Collection}

Gorgonian distribution and abundance. Gorgonian assemblage surveys were conducted between June and September 2009 using SCUBA, snorkeling and scaled digital image photography. Four $10 \mathrm{~m}$ belt $(2 \mathrm{~m}$ either side) transects, laid ca. $20 \mathrm{~m}$ apart, ran parallel to the reef contour at each reef habitat (flat $\leq 3 \mathrm{~m}$, crest ca. $6 \mathrm{~m}$ and slope ca. $12 \mathrm{~m}$ depth) within each site giving a total area surveyed of $1920 \mathrm{~m}^{2}$. Individual colonies encountered along each transect, including beneath canopy structures (see Goatley \& Bellwood 2011), were photographed using a Canon IXUS 900Ti, WP-DC7 u/w housing and INON UWL-105 AD x 0.51 lens. Each image was taken directly opposite and/or above each colony relative to colony morphology with a ruler aligned appropriately for scale. Voucher specimens (2 - $8 \mathrm{~cm}$ in length) were preserved in 96\% EtOH for taxonomic clarification and stored at the Bernice P. Bishop Museum, Honolulu, USA (Accession number: 2014.005). Sclerites were dissolved from the surrounding tissue in 5\% sodium hypochlorite solution and visualized using optical microscopy. Taxonomic identification followed Versluys (1902, 1906), Nutting (1910a-e, 1911), Stiasny (1937, 1940), Aurivillius (1931), Verseveldt (1966), van Ofwegen (1987), Grasshoff (1996, 1999), Grasshoff \& Bargibant (2001), Fabricius \& Alderslade (2001), and Bayer \& Cairns (2004) with most colonies being identified to 'morphotypes' within genera due to the majority of gorgonian species within the Indo-Pacific being undescribed. However, individuals were grouped in accordance with Bayer's (1981) widely accepted three-group (suborders Holaxonia and Calcaxonia, and Scleraxonians group) system, family and genera therein.

Environmental Variables. Sites were characterized through the assessment of 14 (Table 2.1) environmental variables throughout the study period. Benthic characteristics were determined using transects as described for gorgonian surveys, and categorized according to English et al. 
Table 2.1. Environmental characteristics of the four study sites in the Wakatobi Marine National Park, Indonesia. All values expressed as mean $( \pm \mathrm{SE})$ with the exception of diurnal temperature range $\left({ }^{\circ} \mathrm{C}\right)$, light $\left(\mathrm{K}_{\mathrm{d}(\mathrm{PAR})}\right)$ and sediment grain size $(\Phi)$. Abiotic: rock, rubble and sand; biotic: sponges, ascidians, algae (English et al. 1997).

\begin{tabular}{|c|c|c|c|c|}
\hline \multirow{2}{*}{$\begin{array}{l}\text { Parameter Recorded } \\
\text { Site }\end{array}$} & \multicolumn{4}{|c|}{ Mean value \pm SE (where appropriate) } \\
\hline & Sampela & Buoy 3 & Pak Kasim's & Ridge 1 \\
\hline Latitude (S) & $005^{\circ} 29^{\prime} 01^{\prime \prime}$ & $005^{\circ} 28^{\prime} 38^{\prime \prime}$ & $005^{\circ} 27^{\prime} 57^{\prime \prime}$ & $005^{\circ} 26^{\prime} 57^{\prime \prime}$ \\
\hline Longitude $(E)$ & $123^{\circ} 45^{\prime} 08^{\prime \prime}$ & $123^{\circ} 45^{\prime} 47^{\prime \prime}$ & $123^{\circ} 45^{\prime} 18^{\prime \prime}$ & $123^{\circ} 45^{\prime} 38^{\prime \prime}$ \\
\hline Temperature $\left({ }^{\circ} \mathrm{C}\right.$ min-max $)$ & $25.61-29.36$ & $24.69-29.25$ & $26.59-30.457$ & $24.06-28.07$ \\
\hline Light (Kd(PAR) min-max) & $0.31-3.14$ & $0.27-1.96$ & $0.16-2.55$ & $0.1-1.56$ \\
\hline Salinity (PSU) & $32.5 \pm 0.45$ & $33 \pm 0.08$ & $32.8 \pm 0.52$ & $32.6 \pm 0.26$ \\
\hline Flow $(\mathrm{cm} / \mathrm{s})$ & $5.02 \pm 2.18$ & $4.17 \pm 1.35$ & $11.22 \pm 2.55$ & $30.54 \pm 2.61$ \\
\hline Chlorophyll-a ( $\left.\mu \mathrm{g} \mathrm{L}^{1}\right)$ & $0.3 \pm 0.01$ & $0.27 \pm 0.03$ & $0.14 \pm 0.01$ & $0.35 \pm 0.03$ \\
\hline Turbidity (NTU) & $4.38 \pm 1.80$ & $1.04 \pm 0.53$ & $0.54 . \pm 0.72$ & $0.17 \pm 0.33$ \\
\hline Sedimentation $\left(\mathrm{g} \mathrm{d}^{-1}, \mathrm{n}=12\right)$ & $3.28 \pm 0.26$ & $1.52 \pm 0.2$ & $1.23 \pm 0.13$ & $1.16 \pm 0.07$ \\
\hline Sediment grain size $(\Phi, \mathrm{n}=12)$ & $5[31.25-62.5 \mu \mathrm{m}]$ & $1[0.5-1 \mathrm{~mm}]$ & $1[0.5-1 \mathrm{~mm}]$ & $1[0.5-1 \mathrm{~mm}]$ \\
\hline Rugosity Index $(n=12)$ & $0.82 \pm 0.04$ & $0.79 \pm 0.7$ & $0.71 \pm 0.03$ & $0.61 \pm 0.03$ \\
\hline Hard Coral $(\%, n=12)$ & $5.33 \pm 2.04$ & $57.23 \pm 4.6$ & $36.72 \pm 5.11$ & $40.12 \pm 3.1$ \\
\hline Dead Coral/Rubble $(\%, n=12)$ & $38.34 \pm 7.1$ & $10.81 \pm 3.61$ & $12.21 \pm 3.2$ & $6.96 \pm 1.27$ \\
\hline Soft Coral $(\%, n=12)$ & $3.88 \pm 1.42$ & $9.84 \pm 2.91$ & $30.14 \pm 4.85$ & $38.98 \pm 3.83$ \\
\hline Biotic $(\%, n=12)$ & $4.31 \pm 1.21$ & $13.12 \pm 4.43$ & $4.26 \pm 1.65$ & $6.99 \pm 1.44$ \\
\hline Abiotic $(\%, n=12)$ & $48.14 \pm 6.3$ & $9.0 \pm 3.13$ & $16.67 \pm 4.26$ & $6.95 \pm 1.9$ \\
\hline
\end{tabular}

(1997) utilizing the point (every $0.5 \mathrm{~m}$ ) intercept transect method (Kingsford \& Battershill $1998)$. Values are expressed as \% cover ( \pm SE). Rugosity (quantification of habitat complexity) was measured with a $7.30 \mathrm{~m}$ length chain laid over three replicate transects per habitat and calculated using the ratio of contoured surface distance to linear distance method (McCormick 1994).

Suspended sedimentation rates were assessed using four standard 1.0 litre sediment traps (English et al. 1997) deployed at each habitat within all sites for a 10 day period. Sediment and water were filtered (Whatman $0.2 \mu \mathrm{m}$ pore size), dried at $60^{\circ} \mathrm{C}$ and weighed with rates expressed as g dry weight day ${ }^{-1}$. Estimation of sediment grain diameter for all samples was determined using Retsch Technology ${ }^{\circledR}$ test sieves (aperture size range: 2.0, 1, 0.5, 0.125, 0.25, $0.063,<0.063 \mathrm{~mm})$, logarithmically converted, expressed as $p h i(\Phi)$ and classified under the Wentworth scale (Wentworth 1922). Water flow velocity was measured using a General Oceanics ${ }^{\circledR}$ flow meter with a low velocity rotor and custom made aluminum pipes for reef 
placement and expressed as cm/s. Chlorophyll- $a$ (as $\mu \mathrm{g} \mathrm{L}^{-1}$ ), salinity (PSU) and turbidity (NTU) were measured using $\mathrm{RBR}^{\circledR}$ XR-420 CTD data loggers. Temperature $\left({ }^{\circ} \mathrm{C}\right)$ and light $\left(\mathrm{K}_{\mathrm{d}(\mathrm{PAR})}\right)$ were measured using $\mathrm{HOBO}^{\circledR}$ data loggers. The loggers were placed at each transect depth, recording every minute for up to 24 hours. Latitude and longitude were determined by a handheld GPS meter (GARMIN eTrex ${ }^{\circledR}$ ). All variables, with the exception of latitude and longitude, were entered into the statistical models as raw values. Values were edited visually with significant outliers removed.

\subsubsection{Data Analyses}

Data were analyzed using univariate (SPSS v18.0) and multivariate routines in the PRIMER-E v6.1.12 statistical package (Clarke \& Gorley 2006), with PERMANOVA+ v1.02 extension (Anderson 2001). The first hypothesis was to characterise gorgonian diversity, abundance and distribution patterns across gradients of habitat quality within the WMNP. Gorgonian assemblage data were dispersion-weighted, a transformation procedure that accounts for the variance structure of individual species (Clarke et al. 2006). Differences in gorgonian assemblages were analysed according to a two-factor (site and habitat) crossed model with pairwise comparisons using 9999 permutations (PERMANOVA; Anderson 2001) based on a 'zero-adjusted' Bray-Curtis similarity matrix (Clarke et al. 2006b). Results were visualized using non-metric multidimensional scaling (nMDS) ordination comparable with a constrained canonical analysis of principal coordinates (CAP; Anderson \& Willis 2003). Such comparisons reveal real group differences to the maximum variation between groups. The second hypothesis was to identify biologically successful gorgonian species across such environmental clines. In order to test this second hypothesis; prominent taxa contributing to dissimilarities among gorgonian assemblages were investigated using similarity percentages (SIMPER; Clarke 1993). The influence of dominant species revealed from the SIMPER analyses was further investigated using Pearson's product-moment correlations for each species with each canonical axes (Anderson \& Willis 2003) and displayed as a vector overlay on CAP ordinations. Species diversity indices were used across sites and habitats including total number of species $(\mathrm{S})$, the Hill numbers N1, N2 and modified ratio N21' (Peet 1974) to assess the influence of rare and dominant species, and taxonomic spread (equitability) respectively (Clarke \& Gorley 2006). Zooxanthellate and azooxanthellate gorgonian distributions were tested for independence using the Wald-Wolfowitz (runs) test (SPSS v18.0; Wald \& Wolfowitz 1943).

The final hypothesis was to identify predictor environmental variable(s) inferred to influence the ecological structure of gorgonian assemblages. This was investigated using the distance-based 

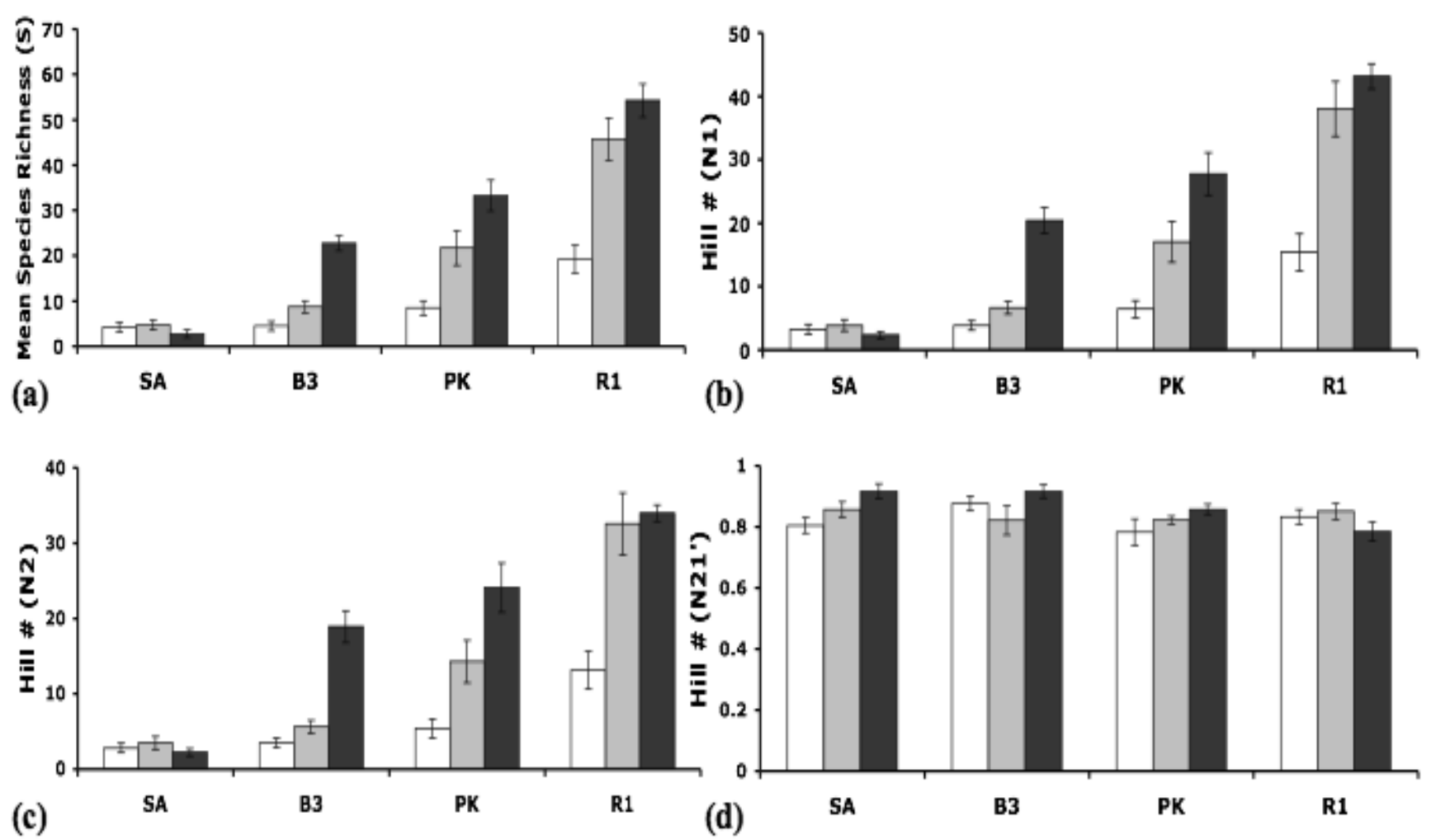

Figure 2.2. Gorgonian species richness (a), Hill's diversity indices N1 (b), N2 (c) and modified ratio for evenness N21' (d) (mean \pm SE) across sites and habitats. Sa, Sampela; B3, Buoy 3; PK, Pak Kasim's; R1, Ridge 1. White bars, reef flat; grey bars, reef crest; black bars, reef slope.

forward selection analysis of linear models (DISTLMforward; McArdle \& Anderson 2001) based on a Euclidean distance matrix. Variables were normalized and conditionally tested using 9999 permutations of the residuals under a reduced model (Anderson 2001). Results were visualized using the distance-based redundancy analysis ordination (dbRDA; McArdle and Anderson 2001).

\subsection{RESULTS}

\subsubsection{Gorgonian Distribution and Abundance}

A total of 3449 gorgonian colonies were documented in this study; 126, 445, 1165 and 1713 recorded at Sampela (impacted), Buoy 3 (intermediate I), Pak Kasim's (intermediate II) and Ridge 1 (healthy) respectively (Figure 2.2; Table 2.2). At present 197 gorgonian species and morphotypes from 37 genera, and 12 families within the suborders/group Calcaxonia, Holaxonia and Scleraxonia have been identified (Table 2.2). This list comprises 21 new species for the region, 28 new species records and another 115 as yet unidentified. Species richness, and diversity followed a typical pattern of increase from the impacted site Sampela to the pristine site Ridge 1, with 7 species at Sampela, 70 species at Buoy3, 80 species at Pak Kasim's and 130 
Table 2.2. Gorgonian species inventory and abundance recorded in this study across sites in the WMNP. Z \& AZ, zooxanthellate and azooxanthellate taxa as classified for statistical analyses. NS, new species; NR, new record for the area; NT, not present on transects but specimen collected; asterisk indicates species status or record unclear requiring further investigation.

\begin{tabular}{|c|c|c|c|c|c|c|}
\hline Taxon & $\mathbf{Z} / \mathbf{A Z}$ & $\begin{array}{c}\text { NR/NS/ } \\
\text { NT }\end{array}$ & Sampela & $\begin{array}{c}\text { Buoy } \\
\mathbf{3}\end{array}$ & $\begin{array}{c}\text { Pak } \\
\text { Kasim's }\end{array}$ & Ridge 1 \\
\hline \multicolumn{7}{|l|}{ [Group: Scleraxonians] } \\
\hline \multicolumn{7}{|l|}{ Family: Anthothelidae Broch 1916} \\
\hline Iciligorgia cf. schrammi Douchassaing 1870 & $\mathrm{AZ}$ & $* \mathrm{NT}$ & - & - & - & - \\
\hline Iciligorgia sp.1 & $\mathrm{AZ}$ & *NT & - & - & - & - \\
\hline Solenocaulon sp.1 & $\mathrm{AZ}$ & $* \mathrm{NT}$ & - & - & - & - \\
\hline Solenocaulon sp.2 & $\mathrm{AZ}$ & $* \mathrm{NT}$ & - & - & - & - \\
\hline \multicolumn{7}{|l|}{ Family: Briareidae Gray 1859} \\
\hline Briareum excavatum Nutting 1911 & $\mathrm{Z}$ & - & 10 & 54 & 168 & 159 \\
\hline Briareum stechei Kükenthal 1908 & $\mathrm{Z}$ & - & 3 & 19 & 35 & 325 \\
\hline Briareum violaceum Roule 1908 & $\mathrm{Z}$ & - & 4 & 1 & - & 15 \\
\hline \multicolumn{7}{|l|}{ Family: Melithaeidae Gray 1870} \\
\hline Acabaria cinquemiglia Grasshoff 1999 & $\mathrm{AZ}$ & NR & - & 2 & 1 & 1 \\
\hline Acabaria variabilis Hickson 1905 & $\mathrm{AZ}$ & - & - & - & 2 & - \\
\hline Acabaria sp.1 & $\mathrm{AZ}$ & $*$ & - & 1 & - & - \\
\hline Acabaria sp. 2 & AZ & $*$ & - & - & - & 1 \\
\hline Acabaria sp. 3 n.sp. & $\mathrm{AZ}$ & NS & - & - & - & 2 \\
\hline Acabaria sp.4 & $\mathrm{AZ}$ & $*$ & - & - & 1 & 8 \\
\hline Acabaria sp.5 & $\mathrm{AZ}$ & * & - & - & - & 6 \\
\hline Acabaria sp.6 & $\mathrm{AZ}$ & $*$ & - & - & - & 2 \\
\hline Acabaria sp.7 & $\mathrm{AZ}$ & $* \mathrm{NT}$ & - & - & - & - \\
\hline Acabaria sp.8 & $\mathrm{AZ}$ & $*$ & - & - & - & 1 \\
\hline Acabaria sp. 9 n.sp. & $\mathrm{AZ}$ & NS & - & - & - & 58 \\
\hline Acabaria sp.10 n.sp. & $\mathrm{AZ}$ & NS & - & - & 1 & 5 \\
\hline Acabaria sp.11 & $\mathrm{AZ}$ & $*$ & - & - & - & 1 \\
\hline Acabaria sp.12 n.sp. & $\mathrm{AZ}$ & NS & - & - & - & 1 \\
\hline Acabaria sp.13 & $\mathrm{AZ}$ & $*$ & - & - & - & 1 \\
\hline Acabaria sp.14 & $\mathrm{AZ}$ & $*$ & - & - & - & 1 \\
\hline Acabaria sp.15 & $\mathrm{AZ}$ & $*$ & - & - & - & 2 \\
\hline Acabaria sp.16 n.sp. & $\mathrm{AZ}$ & NS & - & - & - & 2 \\
\hline Acabaria sp. 17 & $\mathrm{AZ}$ & $*$ & - & - & - & 1 \\
\hline Acabaria sp.18 & $\mathrm{AZ}$ & $*$ & - & 4 & 4 & 12 \\
\hline Acabaria sp.18 n.sp. & $\mathrm{AZ}$ & NS & - & - & 6 & 5 \\
\hline Acabaria sp.19 & $\mathrm{AZ}$ & $*$ & - & 2 & - & 2 \\
\hline Acabaria sp.20 & $\mathrm{AZ}$ & $*$ & - & 2 & - & 7 \\
\hline Acabaria sp.21 & $\mathrm{AZ}$ & * & - & - & - & 1 \\
\hline Acabaria sp. 22 & $\mathrm{AZ}$ & * & - & 1 & - & - \\
\hline Acabaria sp.23 & $\mathrm{AZ}$ & $*$ & - & 10 & - & 13 \\
\hline Acabaria sp.24 n.sp. & $\mathrm{AZ}$ & NS & - & 1 & - & - \\
\hline Acabaria sp. 25 & $\mathrm{AZ}$ & $*$ & - & - & - & 1 \\
\hline Acabaria sp.26 & $\mathrm{AZ}$ & $*$ & - & - & - & 4 \\
\hline Mopsella singularis Thomson 1916 & $\mathrm{AZ}$ & - & - & - & - & 8 \\
\hline Mopsella sp.1 & $\mathrm{AZ}$ & $* \mathrm{NT}$ & - & - & - & - \\
\hline Mopsella sp. 2 & $\mathrm{AZ}$ & $*$ & - & - & - & 1 \\
\hline Mopsella sp.3 & $\mathrm{AZ}$ & $*$ & - & - & - & 4 \\
\hline Melithaea ochracea Linnaeus 1758 & $\mathrm{AZ}$ & - & 1 & - & - & - \\
\hline Melithaea squamata Nutting 1911 & $\mathrm{AZ}$ & - & - & 2 & - & 5 \\
\hline Melithaea sp.1 & $\mathrm{AZ}$ & $*$ & - & - & - & 6 \\
\hline Melithaea sp.2 & $\mathrm{AZ}$ & $*$ & - & - & - & 7 \\
\hline Melithaea sp.3 & $\mathrm{AZ}$ & $*$ & - & 1 & - & 18 \\
\hline
\end{tabular}




\begin{tabular}{|c|c|c|c|c|c|c|}
\hline Taxon & $\mathbf{Z} / \mathbf{A Z}$ & $\begin{array}{c}\text { NR/NS/ } \\
\text { NT }\end{array}$ & Sampela & $\begin{array}{c}\text { Buoy } \\
\mathbf{3}\end{array}$ & $\begin{array}{c}\text { Pak } \\
\text { Kasim's }\end{array}$ & Ridge 1 \\
\hline Melithaea sp.4 & $\mathrm{AZ}$ & $*$ & - & - & - & 2 \\
\hline Melithaea sp.5 & $\mathrm{AZ}$ & $*$ & - & 1 & - & 1 \\
\hline Melithaea sp.6 & $\mathrm{AZ}$ & $*$ & - & - & - & 3 \\
\hline Melithaea sp.7 & $\mathrm{AZ}$ & $* \mathrm{NT}$ & - & - & - & - \\
\hline Melithaea sp. 8 & $\mathrm{AZ}$ & * & - & - & - & 3 \\
\hline Melithaea sp.9 & $\mathrm{AZ}$ & * & - & - & - & 3 \\
\hline \multicolumn{7}{|l|}{ Family: Parisididae Aurivillius 1931} \\
\hline Parisis sp.1 & $\mathrm{AZ}$ & * & - & - & - & 1 \\
\hline \multicolumn{7}{|l|}{ Family: Subergorgiidae Gray 1859} \\
\hline Annella mollis Nutting 1910 & $\mathrm{AZ}$ & - & - & - & - & 3 \\
\hline Annella reticulata Ellis \& Solander 1736 & $\mathrm{AZ}$ & - & - & 2 & 10 & 14 \\
\hline Annella sp.1 n.sp. & $\mathrm{AZ}$ & NS & - & 9 & - & 17 \\
\hline Annella sp.2 n.sp. & $\mathrm{AZ}$ & NS & - & - & 4 & 5 \\
\hline Subergorgia rubra Gray 1857 & $\mathrm{AZ}$ & NT & - & - & - & - \\
\hline Subergorgia suberosa Pallas 1766 & $\mathrm{AZ}$ & NT & - & - & - & - \\
\hline \multirow{2}{*}{\multicolumn{7}{|c|}{ [Suborder: Holaxonians] }} \\
\hline & & & & & & \\
\hline \multicolumn{7}{|l|}{ Family: Keroeididae Kinshita 1910} \\
\hline Keroeides cf. gracilis Whitelegge 1897 & $\mathrm{AZ}$ & * & - & 1 & - & - \\
\hline \multicolumn{7}{|l|}{ Family: Gorgoniidae Lamouroux 1812} \\
\hline Guaiagorgia sp.1 & $\mathrm{AZ}$ & $* \mathrm{NR} / \mathrm{NT}$ & - & - & - & - \\
\hline Hicksonella princeps Nutting 1910 & $\mathrm{Z}$ & * & - & - & - & 1 \\
\hline Pinnigorgia sp.1 & $\mathrm{Z}$ & * & - & - & 2 & 1 \\
\hline Pseudopterogorgia sp.1 & $\mathrm{AZ}$ & $*$ & - & - & - & 1 \\
\hline Rumphella aggregata Nutting 1910 & $\mathrm{Z}$ & - & 3 & - & 1 & 5 \\
\hline Rumphella antipathes Linnaeus 1758 & $\mathrm{Z}$ & NR & - & - & - & 1 \\
\hline Rumphella sp.1 & $\mathrm{Z}$ & $*$ & - & - & 2 & - \\
\hline \multicolumn{7}{|l|}{ Family: Acanthogorgiidae Gray 1859} \\
\hline Acanthogorgia cf. isoyxa Grasshoff 1999 & $\mathrm{AZ}$ & NR & - & 2 & - & 6 \\
\hline Acanthogorgia spinosa Hiles 1899 & $\mathrm{AZ}$ & - & - & 1 & 6 & 9 \\
\hline Acanthogorgia sp.1 n.sp. & $\mathrm{AZ}$ & NS & - & 1 & - & - \\
\hline Acanthogorgia sp.2 n.sp. & $\mathrm{AZ}$ & NS & - & 82 & 7 & 5 \\
\hline Acanthogorgia sp. 3 & $\mathrm{AZ}$ & $*$ & - & - & - & 1 \\
\hline Acanthogorgia sp.4 & $\mathrm{AZ}$ & * & - & 3 & 12 & 9 \\
\hline Acanthogorgia sp.5 & $\mathrm{AZ}$ & $*$ & - & 1 & 14 & 8 \\
\hline Acanthogorgia sp.6 n.sp. & $\mathrm{AZ}$ & NS & - & - & 3 & 12 \\
\hline Acanthogorgia sp.7 & $\mathrm{AZ}$ & $*$ & - & 1 & 1 & 1 \\
\hline Acanthogorgia sp.8 & $\mathrm{AZ}$ & $*$ & - & - & 1 & - \\
\hline Acanthogorgia sp.9 & $\mathrm{AZ}$ & $*$ & - & - & - & 1 \\
\hline Acanthogorgia sp.10 & $\mathrm{AZ}$ & * & - & - & 1 & 1 \\
\hline Acanthogorgia sp.11 & $\mathrm{AZ}$ & $*$ & - & - & 1 & 2 \\
\hline Anthogorgia sp.1 & $\mathrm{AZ}$ & *NT & - & - & - & - \\
\hline Muricella sp.1 & $\mathrm{AZ}$ & $*$ & - & 2 & 2 & - \\
\hline Muricella sp.2 & $\mathrm{AZ}$ & $* \mathrm{NT}$ & - & - & - & - \\
\hline \multicolumn{7}{|l|}{ Family: Plexauridae Gray 1859} \\
\hline Acanthomuricea sp.1 & $\mathrm{AZ}$ & * & - & 1 & 1 & - \\
\hline Astrogorgia bayeri van Ofwegen \& Hoeksema 2001 & $\mathrm{AZ}$ & NR/NT & - & - & - & - \\
\hline Astrogorgia canala Grasshoff 1999 & $\mathrm{AZ}$ & NR & - & - & 6 & 11 \\
\hline Astrogorgia dumbea Grasshoff 1999 & $\mathrm{AZ}$ & NR & - & - & 1 & 3 \\
\hline Astrogorgia cf. arborea Thomson \& Simpson 1909 & $\mathrm{AZ}$ & *NT & - & - & - & - \\
\hline Astrogorgia sp.1 & $\mathrm{AZ}$ & $* \mathrm{NT}$ & - & - & - & - \\
\hline Astrogorgia sp. 2 & $\mathrm{AZ}$ & $*$ & - & 1 & 1 & - \\
\hline Astrogorgia sp.3 & $\mathrm{AZ}$ & $*$ & - & - & - & 4 \\
\hline Astrogorgia sp.6 n.sp. & $\mathrm{AZ}$ & NS & - & 15 & 135 & 96 \\
\hline Astrogorgia sp.7 & $\mathrm{AZ}$ & $*$ & - & - & 13 & 11 \\
\hline Astrogorgia sp.8 n.sp. & $\mathrm{AZ}$ & NS & - & - & 2 & 7 \\
\hline Astrogorgia sp.9 n.sp. & $\mathrm{AZ}$ & NS & - & 3 & - & 9 \\
\hline
\end{tabular}




\begin{tabular}{|c|c|c|c|c|c|c|}
\hline Taxon & $\mathbf{Z} / \mathbf{A Z}$ & $\begin{array}{c}\text { NR/NS/ } \\
\text { NT }\end{array}$ & Sampela & $\begin{array}{c}\text { Buoy } \\
\mathbf{3}\end{array}$ & $\begin{array}{c}\text { Pak } \\
\text { Kasim's }\end{array}$ & Ridge 1 \\
\hline Astrogorgia sp.10 & $\mathrm{AZ}$ & $*$ & - & 5 & 9 & 29 \\
\hline Astrogorgia sp.11 & $\mathrm{AZ}$ & $*$ & - & - & 3 & 10 \\
\hline Astrogorgia sp.12 & $\mathrm{AZ}$ & $*$ & - & - & - & 1 \\
\hline Astrogorgia sp.13 & $\mathrm{AZ}$ & $* \mathrm{NT}$ & - & - & - & - \\
\hline Astrogorgia sp.13 & $\mathrm{AZ}$ & $*$ & - & - & 8 & 23 \\
\hline Astrogorgia sp.14 & $\mathrm{AZ}$ & $* \mathrm{NT}$ & - & - & - & - \\
\hline Astrogorgia sp.15 n.sp. & $\mathrm{AZ}$ & NS & - & - & 1 & 6 \\
\hline Astrogorgia sp.16 & $\mathrm{AZ}$ & $* \mathrm{NT}$ & - & - & - & - \\
\hline Astrogorgia sp.17 & $\mathrm{AZ}$ & $*$ & - & 1 & 24 & 14 \\
\hline Astrogorgia sp.18 & $\mathrm{AZ}$ & $*$ & - & 20 & 19 & 43 \\
\hline Astrogorgia sp.19 & $\mathrm{AZ}$ & $*$ & 1 & - & - & - \\
\hline Astrogorgia sp.20 & $\mathrm{AZ}$ & $*$ & - & 1 & 19 & 22 \\
\hline Astrogorgia sp. 21 & $\mathrm{AZ}$ & $*$ & - & - & 4 & 1 \\
\hline Astrogorgia sp.22 & $\mathrm{AZ}$ & $*$ & - & - & 1 & 1 \\
\hline Astrogorgia sp.23 n.sp. & $\mathrm{AZ}$ & NS & - & 3 & 5 & 11 \\
\hline Bebryce hicksoni Thomson \& Henderson 1905 & $\mathrm{AZ}$ & - & - & 3 & 1 & 3 \\
\hline Bebryce cf. indica Thomson 1905 & $\mathrm{AZ}$ & $*$ & - & 6 & 16 & 59 \\
\hline Bebryce thomsoni Nutting 1910 & $\mathrm{AZ}$ & - & - & - & 1 & 3 \\
\hline Bebryce sp.1 & $\mathrm{AZ}$ & *NT & - & - & - & - \\
\hline Bebryce sp.2 & $\mathrm{AZ}$ & *NT & - & - & - & - \\
\hline Bebryce sp. 3 & $\mathrm{AZ}$ & *NT & - & - & - & - \\
\hline Echinogorgia furfuracea Esper 1791 & $\mathrm{AZ}$ & NR & - & - & - & 2 \\
\hline Echinogorgia cf. furfuracea Esper 1791 & $\mathrm{AZ}$ & $*$ & - & 1 & 3 & 3 \\
\hline Echinogorgia pseudosassapo Kölliker 1865 & $\mathrm{AZ}$ & NR & - & - & 1 & - \\
\hline Echinogorgia sp.1 n.sp. & $\mathrm{AZ}$ & NS & - & - & 1 & - \\
\hline Echinogorgia sp.2. n.sp & $\mathrm{AZ}$ & NS & - & 1 & - & - \\
\hline Echinogorgia sp.3 n.sp. & $\mathrm{AZ}$ & NS & - & - & - & 1 \\
\hline Echinogorgia sp.4 & $\mathrm{AZ}$ & $*$ & - & 1 & 2 & 3 \\
\hline Echinogorgia sp.5 & $\mathrm{AZ}$ & $*$ & - & - & - & 1 \\
\hline Echinogorgia sp.6 & $\mathrm{AZ}$ & $* \mathrm{NT}$ & - & - & - & - \\
\hline Echinogorgia sp.7 & $\mathrm{AZ}$ & *NT & - & - & - & - \\
\hline Echinogorgia sp. 8 & AZ & $*$ & - & - & - & 1 \\
\hline Paracis rigida Thomson \& Simpson 1909 & $\mathrm{AZ}$ & NR & - & - & 2 & - \\
\hline Paracis sp. 1 n.sp & $\mathrm{AZ}$ & NS & - & - & - & 1 \\
\hline Paracis sp. 2 & $\mathrm{AZ}$ & $* \mathrm{NT}$ & - & - & - & - \\
\hline Paracis sp.3 & $\mathrm{AZ}$ & $*$ & - & - & 1 & 1 \\
\hline Echinomuricea cf. coronalis Germanos 1896 & $\mathrm{AZ}$ & NR & - & 1 & - & - \\
\hline Echinomuricea indomalaccensis Ridley 1884 & $\mathrm{AZ}$ & - & - & 2 & 8 & 7 \\
\hline Echinomuricea ochracea Thomson \& Simpson 1909 & $\mathrm{AZ}$ & NR & - & 1 & - & - \\
\hline Echinomuricea pulchra Nutting 1910 & $\mathrm{AZ}$ & NR & - & - & 2 & 2 \\
\hline Echinomuricea splendens Thomson \& Simpson 1909 & $\mathrm{AZ}$ & - & - & - & 5 & 1 \\
\hline Echinomuricea sp.1 n.sp. & $\mathrm{AZ}$ & NS & - & - & - & 1 \\
\hline Echinomuricea sp.2 n.sp. & $\mathrm{AZ}$ & NS/NT & - & - & - & - \\
\hline Echinomuricea sp. 3 & $\mathrm{AZ}$ & $*$ & - & - & 6 & 13 \\
\hline Echinomuricea sp. 4 & $\mathrm{AZ}$ & $*$ & - & - & - & 1 \\
\hline Euplexaura rhipidalis Studer 1895 & $\mathrm{AZ}$ & NR & - & 1 & - & 4 \\
\hline Euplexaura sp.1 & $\mathrm{AZ}$ & $*$ & - & 1 & 3 & 2 \\
\hline Euplexaura sp.2 & $\mathrm{AZ}$ & $*$ & - & - & - & 1 \\
\hline Euplexaura sp.3 n.sp. & $\mathrm{AZ}$ & NS & - & 2 & 1 & - \\
\hline Euplexaura sp.4 & $\mathrm{AZ}$ & $*$ & - & - & - & 1 \\
\hline Euplexaura sp.5 & $\mathrm{AZ}$ & $*$ & - & - & 2 & - \\
\hline Euplexaura sp.6 & $\mathrm{AZ}$ & $* \mathrm{NT}$ & - & - & - & - \\
\hline Euplexaura sp.7 & $\mathrm{AZ}$ & $*$ & - & - & 1 & - \\
\hline Euplexaura sp. 8 & $\mathrm{AZ}$ & $*$ & - & - & - & 1 \\
\hline Euplexaura sp.9 & $\mathrm{AZ}$ & $*$ & - & - & 2 & - \\
\hline Euplexaura sp.10 & $\mathrm{AZ}$ & $*$ & - & 1 & 1 & 1 \\
\hline
\end{tabular}




\begin{tabular}{|c|c|c|c|c|c|c|}
\hline Taxon & $\mathbf{Z} / \mathbf{A Z}$ & $\begin{array}{c}\text { NR/NS/ } \\
\text { NT }\end{array}$ & Sampela & $\begin{array}{c}\text { Buoy } \\
\mathbf{3}\end{array}$ & $\begin{array}{c}\text { Pak } \\
\text { Kasim's }\end{array}$ & Ridge 1 \\
\hline Menella indica Ridley 1888 & $\mathrm{AZ}$ & NR & - & 1 & - & - \\
\hline Menella lenzii Studer 1895 & $\mathrm{AZ}$ & NR & - & 1 & 3 & 11 \\
\hline Menella praelonga Ridley 1884 & $\mathrm{AZ}$ & - & - & 1 & 1 & \\
\hline Menella spinifera Kükenthal 1911 & $\mathrm{AZ}$ & - & - & 1 & 6 & 13 \\
\hline Menella sp.1 & $\mathrm{AZ}$ & * & - & 1 & 4 & 2 \\
\hline Menella sp. 2 & $\mathrm{AZ}$ & $*$ & - & 1 & - & 3 \\
\hline Menella sp.3 & $\mathrm{AZ}$ & $*$ & - & - & - & 3 \\
\hline Trimuricea sp. 1 n.sp. & $\mathrm{AZ}$ & NS/NT & - & - & - & - \\
\hline Trimuricea sp. 2 n.sp. & $\mathrm{AZ}$ & NS & - & - & 1 & - \\
\hline Paraplexaura cf. cimenia Grasshoff 1999 & $\mathrm{AZ}$ & NR & - & - & - & 1 \\
\hline Paraplexaura sp.1 & $\mathrm{AZ}$ & $*$ & - & 4 & 4 & - \\
\hline Paraplexaura sp.2 & $\mathrm{AZ}$ & * & - & 1 & 4 & 1 \\
\hline Paraplexaura sp. 3 & $\mathrm{AZ}$ & * & - & - & 6 & 19 \\
\hline Paraplexaura sp.4 & $\mathrm{AZ}$ & $*$ & - & 1 & - & - \\
\hline Villogorgia cf. citrina Grasshoff 1999 & $\mathrm{AZ}$ & $* \mathrm{NR}$ & - & 1 & - & 7 \\
\hline Villogorgia rubra Nutting 1910 & $\mathrm{AZ}$ & - & - & - & 1 & 2 \\
\hline Villogorgia sp.1 n.sp. & $\mathrm{AZ}$ & NS & - & 2 & 1 & 9 \\
\hline Villogorgia sp.2 & $\mathrm{AZ}$ & $*$ & - & - & 1 & 3 \\
\hline Villogorgia sp.3 & $\mathrm{AZ}$ & $*$ & - & - & - & 3 \\
\hline Villogorgia sp.4 & $\mathrm{AZ}$ & $* \mathrm{NT}$ & - & - & - & - \\
\hline Villogorgia sp.5 & $\mathrm{AZ}$ & *NT & - & - & - & - \\
\hline [Suborder: Calcaxonians] & & - & - & & & \\
\hline \multicolumn{7}{|l|}{ Family: Ellisellidae Gray 1859} \\
\hline Ctenocella sp.1 & $\mathrm{AZ}$ & *NT & - & - & - & - \\
\hline Ellisella ceratophyta Linnaeus 1758 & $\mathrm{AZ}$ & - & - & 1 & - & 16 \\
\hline Ellisella plexauroides Toeplitz 1919 & $\mathrm{AZ}$ & - & - & - & - & 8 \\
\hline Ellisella sp.1 & $\mathrm{AZ}$ & $*$ & - & 2 & - & 5 \\
\hline Ellisella sp.2 & $\mathrm{AZ}$ & $*$ & - & 1 & 1 & 5 \\
\hline Ellisella sp. 3 & $\mathrm{AZ}$ & * & - & - & 2 & 1 \\
\hline Dichotella gemmacea Milne Edwards \& Haime 1857 & $\mathrm{AZ}$ & $*$ & - & - & 6 & 3 \\
\hline Heliania sp.1 & $\mathrm{AZ}$ & $* \mathrm{NR} / \mathrm{NT}$ & - & - & - & - \\
\hline Junceella fragilis Ridley 1884 & $\mathrm{AZ}$ & - & - & 2 & - & 16 \\
\hline Junceella cf. juncea Pallas 1766 & $\mathrm{AZ}$ & * & - & - & - & 1 \\
\hline Nicella sp.1 & $\mathrm{AZ}$ & *NT & - & - & - & - \\
\hline Verrucella cf. cerasina Grasshoff 1999 & $\mathrm{AZ}$ & $* \mathrm{NR}$ & - & 2 & 1 & 5 \\
\hline Verrucella cf. rubra Nutting 1910 & $\mathrm{AZ}$ & $* \mathrm{NR} / \mathrm{NT}$ & - & - & - & - \\
\hline Verrucella sp.1 & $\mathrm{AZ}$ & * & - & 4 & 3 & 3 \\
\hline Verrucella sp.2 & $\mathrm{AZ}$ & *NT & - & - & - & - \\
\hline Viminella sp.1 & $\mathrm{AZ}$ & $*$ & - & - & - & 1 \\
\hline \multicolumn{7}{|l|}{ Family: Ifalukellidae Bayer 1955} \\
\hline Ifalukella yanii Bayer 1955 & $\mathrm{Z}$ & NT & - & - & - & - \\
\hline Plumigorgia hydroides Nutting 1910 & $\mathrm{Z}$ & NT & - & - & - & - \\
\hline Plumigorgia schuboti Alderslade 1986 & $\mathrm{Z}$ & NT & - & 1 & - & - \\
\hline \multicolumn{7}{|l|}{ Family: Isididae Lamouroux 1812} \\
\hline Isis hippuris $[\mathrm{N}]$ Linnaeus 1758 & $\mathrm{Z}$ & - & 41 & 103 & 413 & 278 \\
\hline Isis hippuris[LT] Linnaeus 1758 & $\mathrm{Z}$ & - & 59 & 7 & 71 & 49 \\
\hline Isis hippuris[S] Linnaeus 1758 & $\mathrm{Z}$ & - & 4 & 20 & 26 & 15 \\
\hline Zignisis sp.1 & $\mathrm{AZ}$ & $* \mathrm{NR}$ & - & 1 & - & - \\
\hline Unidentified & $\mathrm{AZ}$ & * & - & 5 & 10 & 7 \\
\hline Unidentified, Holaxonian & $\mathrm{AZ}$ & *NT & - & - & - & - \\
\hline Unidentified, Holaxonian, Plexauridae & $\mathrm{AZ}$ & * & - & 4 & 4 & 6 \\
\hline Total \# Species: & & & 126 & 445 & 1165 & 1713 \\
\hline
\end{tabular}




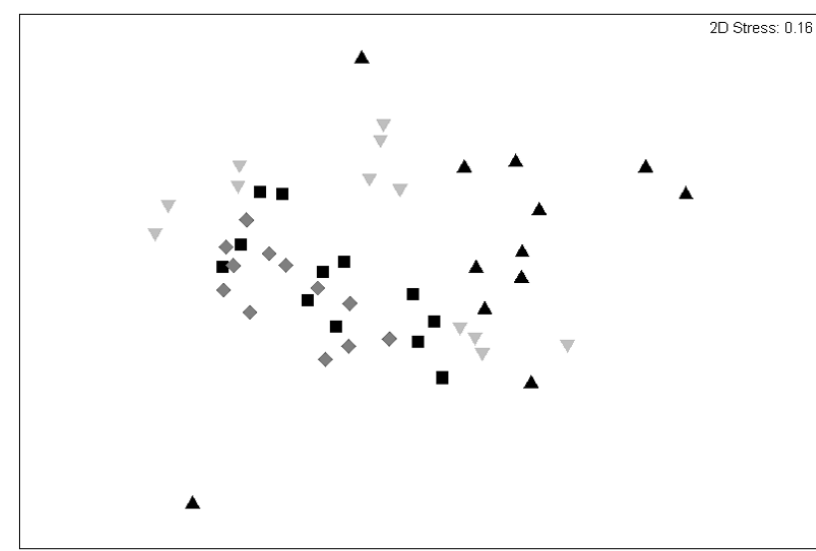

(a)

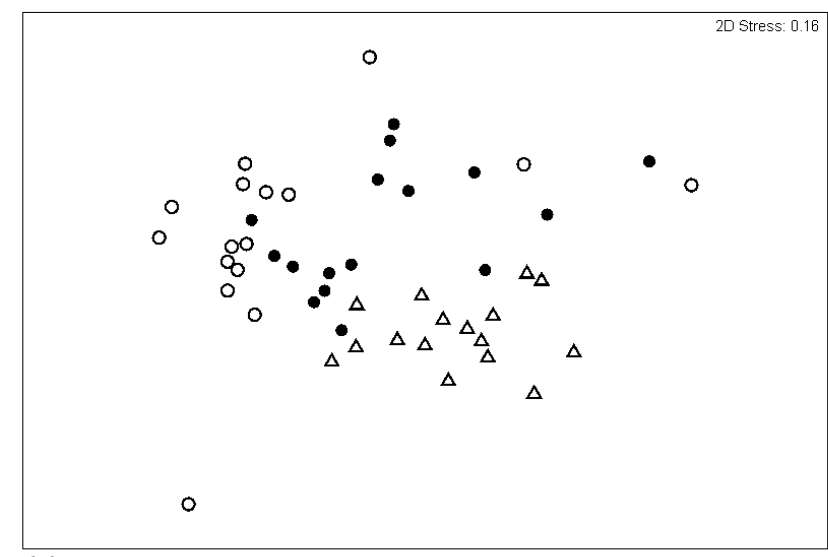

(c)

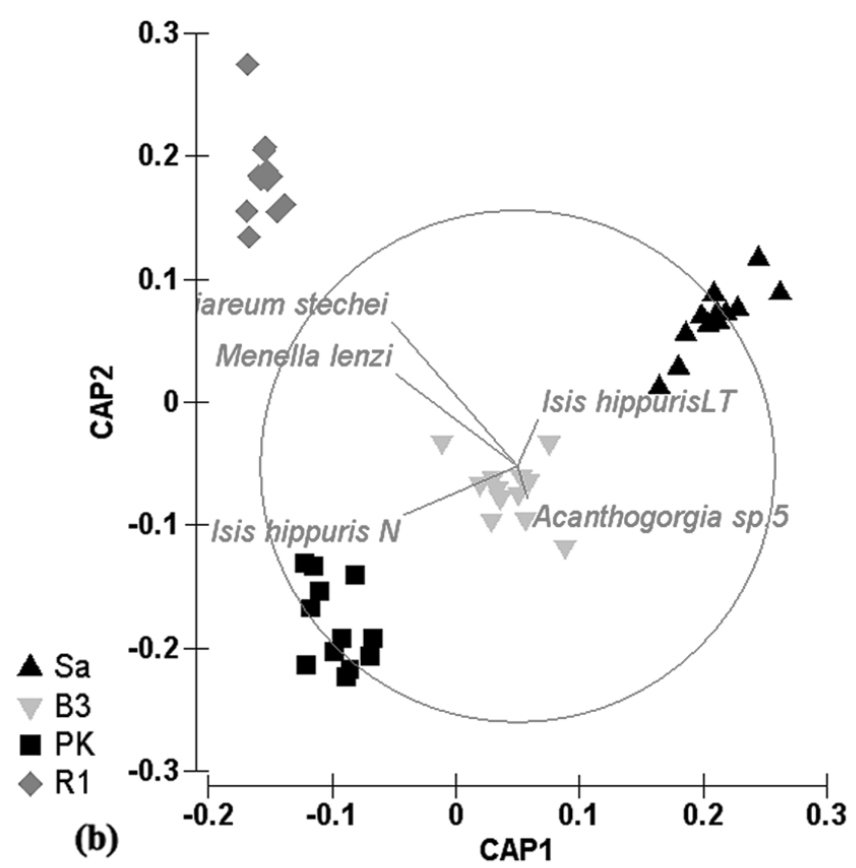

(b)

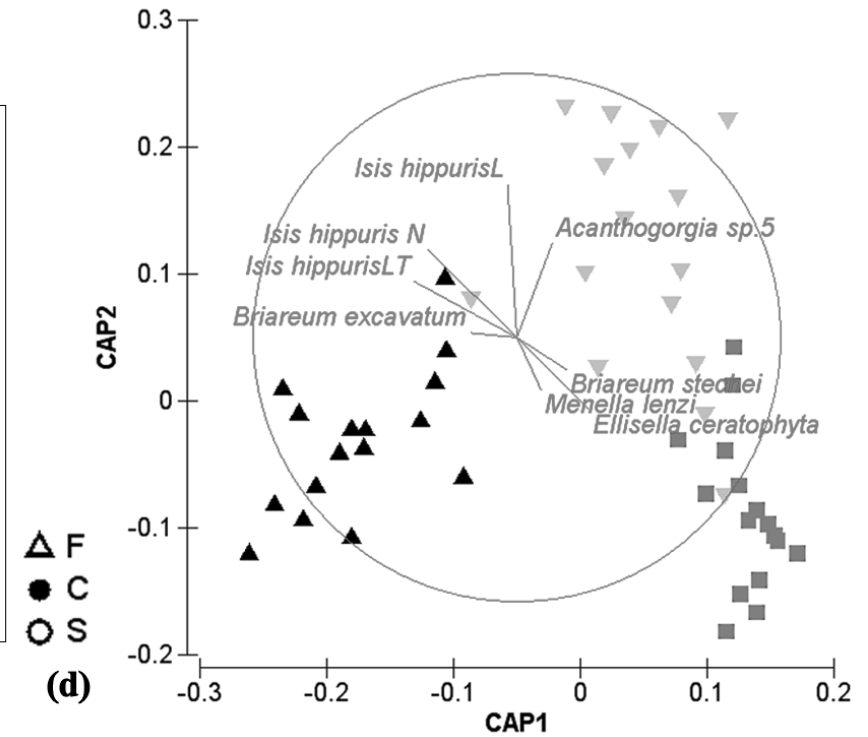

Figure 2.3. (a, c) Constrained and (b, d) unconstrained ordinations of gorgonian assemblages between sites (a, b) and habitats (c, d). Sa, Sampela; B3, Buoy 3; PK, Pak Kasim's; R1, Ridge 1. F, reef flat; $\mathrm{C}$, reef crest; $\mathrm{S}$, reef slope.

species at Ridge 1 (Figure 2.2; Table 2.2). This pattern of increased species richness and diversity was similarly replicated with depth, the inverse evident in Sampela with the majority of colonies and species on the reef crest and flat (Figure 2.2).

PERMANOVA results revealed that differences in gorgonian abundance across all sites and habitats were significant with no interaction effects (pseudo- $F=7.938, P<0.0001$; pseudo- $F=$ 6.714, $P<0.0001)$. Pair wise comparisons revealed significant differences were between all 

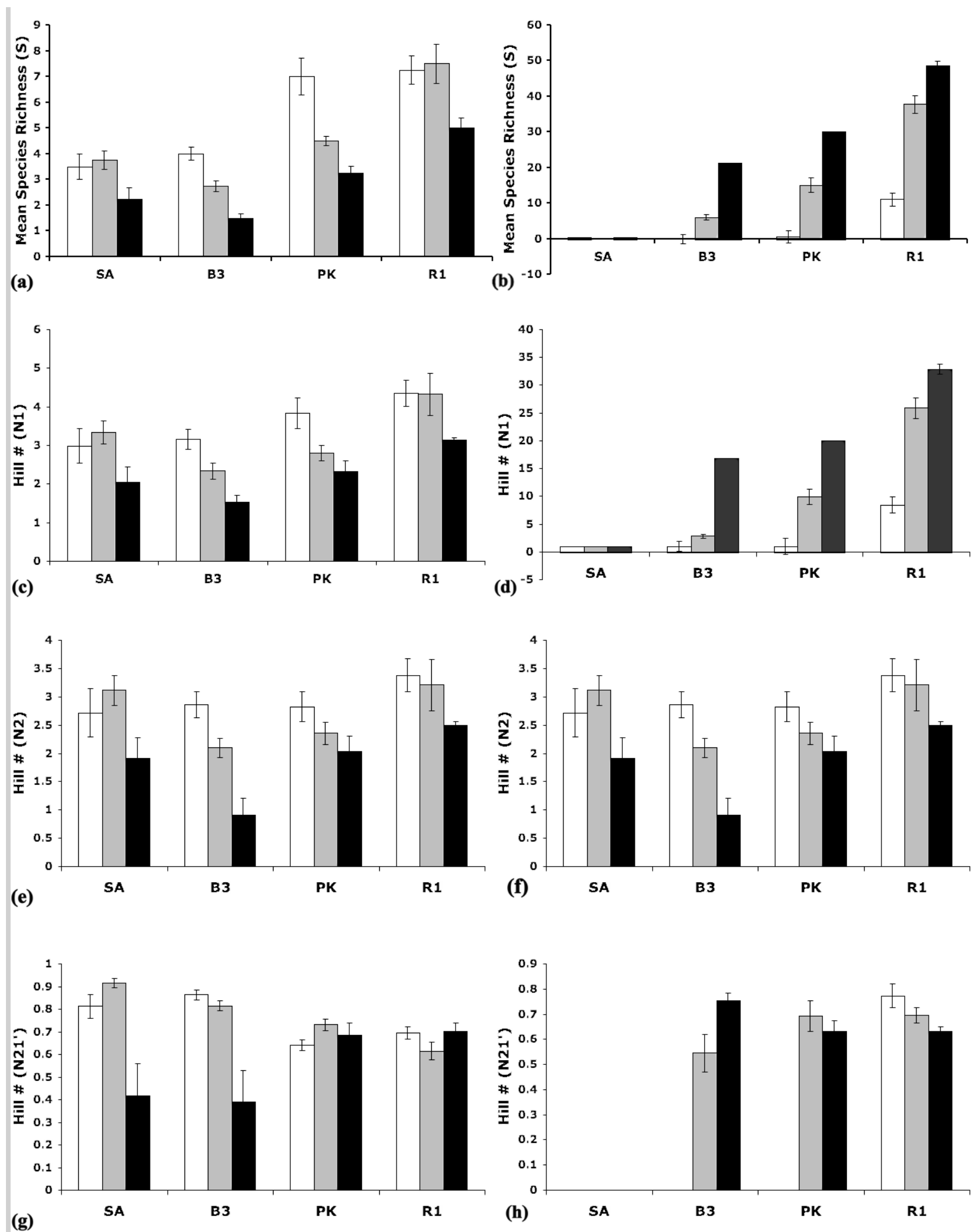

Figure 2.4. Zooxanthellate (a, c, e, g) and azooxanthellate gorgonian species richness (a b), Hill's diversity indices N1 (c, d), N2 (e, f) and modified ratio for evenness N21' (g, h) (mean \pm SE) across sites and habitats. Sa, Sampela; B3, Buoy 3; PK, Pak Kasim's; R1, Ridge 1 within the WMNP. White bars, reef flat; grey bars, reef crest; black bars, reef slope. 
sites and habitats, most notably Sampela and Ridge 1, and the reef flat and slope respectively (Figure 2.3). CAP analyses were consistent with these results, where strong allocation success clearly defined distinct assemblage variability between sites and habitats (Figure 2.3; Table 2.2). SIMPER further revealed that 'morphotypes' within the zooxanthellate taxa Isis hippuris [LT,N] Linnaeus 1758 and Briareum excavatum Nutting 1911 accounted most for differences between both site and habitat gorgonian assemblages (Table 2.4). I. hippuris colonies with long thick branches were prevalent on the reef flat at Sampela (Figure 2.3b, d, Table 2.4), whereas lowlying branching Briareum species more abundant towards the reef slope. In addition, the azooxanthellate Acanthogorgia sp.5 contributed considerably towards the difference between the reef crest and flat (Figure 2.3d, Table 2.4). This was due to its exclusive and abundant presence on the ceilings of caves and overhangs, characteristic of Buoy 3.

Zooxanthellate versus Azooxanthellate Gorgonians. The dominance of the zooxanthellate gorgonians I. hippuris (1094) and Briareum spp. (792) obscured distribution patterns of azooxanthellate taxa (Figure 2.3). To highlight indicative distribution patterns and potential interactions between zooxanthellate and azooxanthellate gorgonian assemblages and their environment, tests of 1) diversity and richness; 2) independence; 3) separate assemblage structure, and 4) environmental driver(s) were performed.

A total of 1900 zooxanthellate and 1517 azooxanthellate gorgonian colonies were surveyed across reefs within the WMNP. Calcaxonians, holaxonians, as well as scleraxonians were represented by both zooxanthellate and azooxanthellate taxa with 6 genera belonging to 4 families, and 31 genera belonging to 9 families respectively. Taxonomic richness and diversity for azooxanthellate species largely replicated that of figure 2.2 - all taxa (Figure 2.4b, d, f, h); increasing towards Ridge 1 and with depth. Zooxanthellate taxonomic richness and diversity also increased with site, however showed an inverse relationship with depth, being greatest at the reef crest and flat (Figure 2.4a, c, e, g).

Results from a Wald-Wolfowitz (runs) test revealed the distributions of zooxanthellate and azooxanthellate taxa were non-random, rejecting the null hypothesis of independence $(P<$ 0.001). The relative abundance of both zooxanthellate and azooxanthellate taxa differed significantly across sites (PERMANOVA, pseudo- $F=9.476, P<0.0001$ and pseudo- $F=3.997$; $P<0.0001$, respectively) and habitats (PERMANOVA, pseudo- $F=7.716, P<0.0001$ and pseudo- $F=4.687, P<0.0001$, respectively). Yet an interaction effect (pseudo- $F=1.925 ; P=$ 0.012 ) between sites and habitats for azooxanthellate taxa revealed that significance levels were 


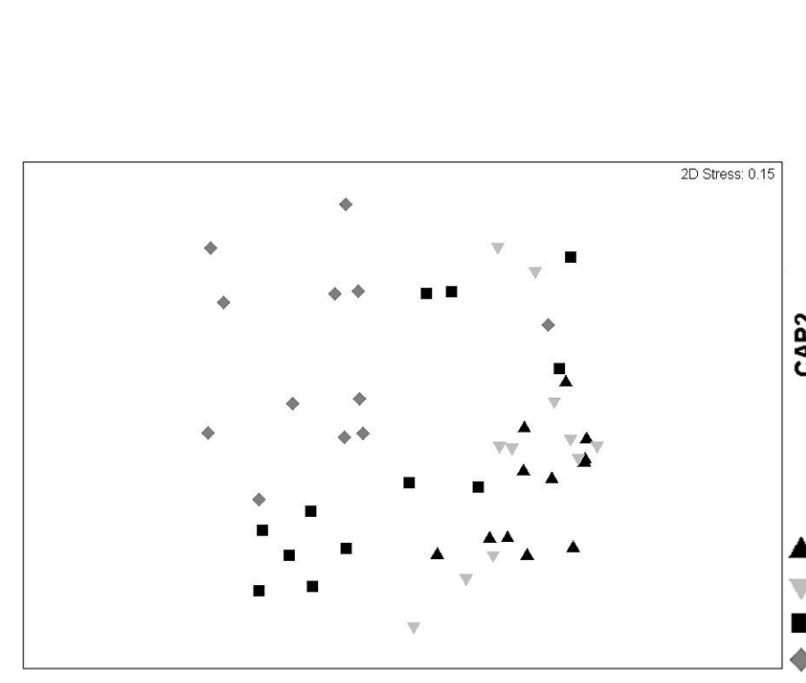

(a)

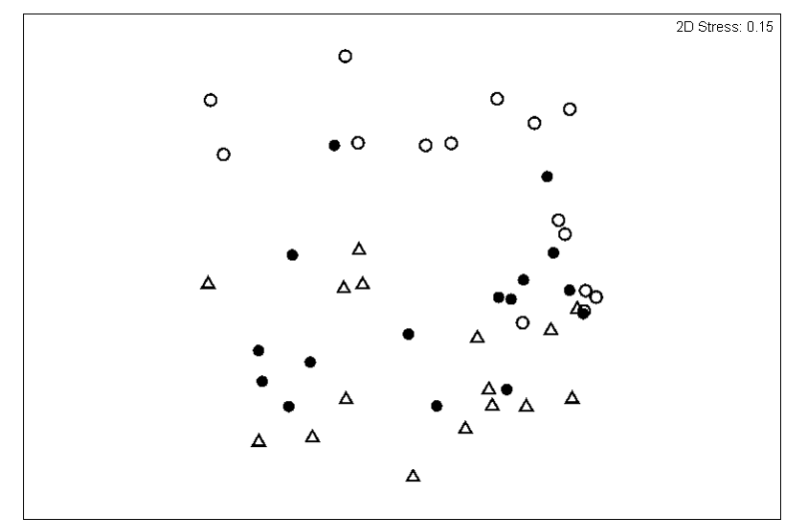

(c)

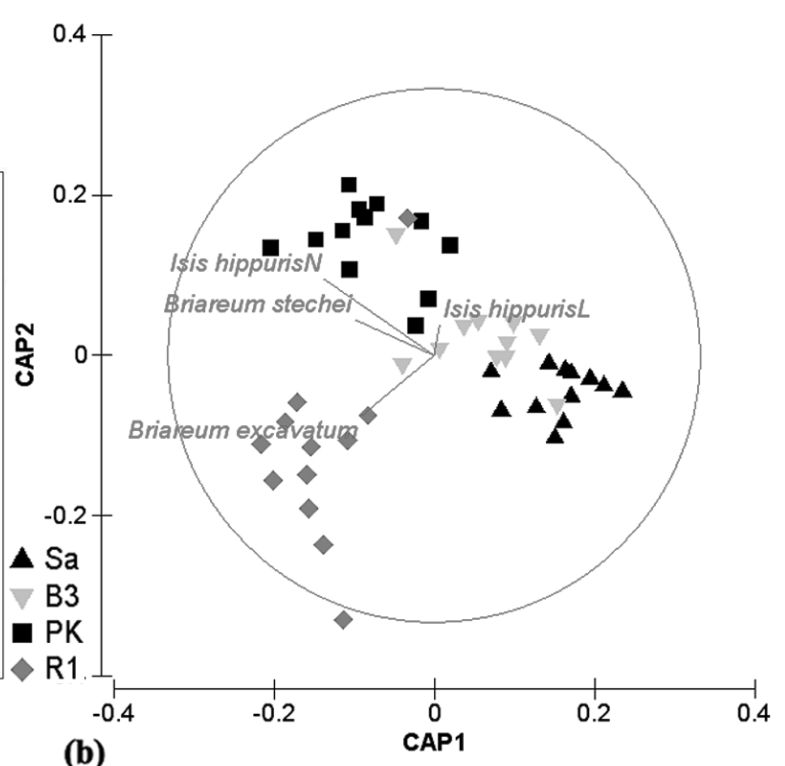

(b)

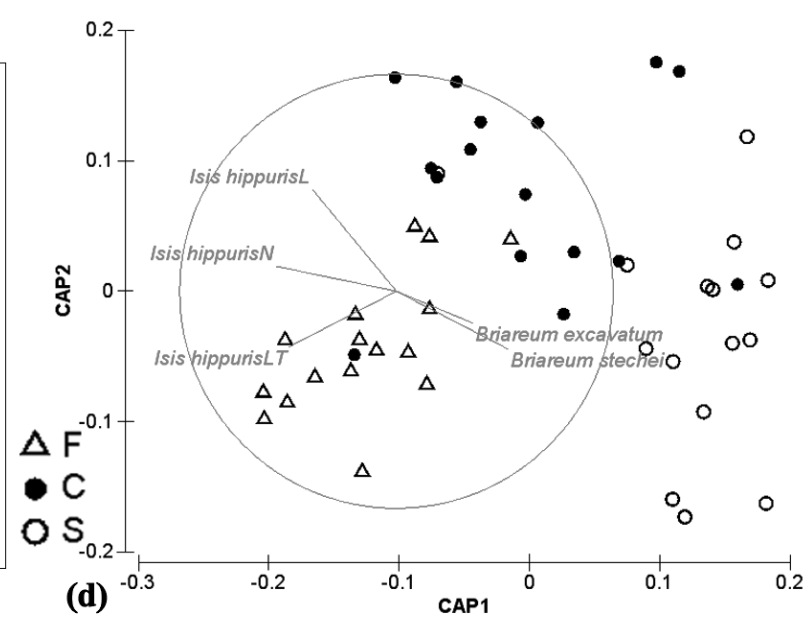

Figure 2.5. ( $a, c)$ Constrained and (b, d) unconstrained ordinations of zooxanthellate gorgonian assemblages between sites $(\mathrm{a}, \mathrm{b})$ and habitats $(\mathrm{c}, \mathrm{d})$. Species vectors are directed where the species were best represented. See Figure 2.3 for factor level codes.

principally driven by zooxanthellate gorgonians. Results were further supported by CAP analyses; allocation success (number of correct allocations to each factor level) was weaker for azooxanthellate taxa at Pak Kasim's, Buoy 3, and the reef crest (Table 2.2). CAP and SIMPER analyses confirmed previous results of $I$. hippuris[LT] on the reef flats at Sampela, I. hippuris $[\mathrm{N}]$ and I. hippuris $[\mathrm{L}]$ towards the reef crest (Figure 2.5, Table $2.5 \& 6$ ). Briareum spp. followed a typical pattern of encrusting on the reef flats at Ridge 1, with low-lying branching colonies characterizing the reef crest and slope, a pattern particularly replicated at Pak Kasim's (Figure 2.5, Table 2.5). It is notable that Briareum spp. and I. hippuris colonies altered in colouration (magenta to brown or grey and mustard-yellow to beige respectively) at depth and areas of high turbidity. 
Table 2.3. CAP analyses results assessing gorgonian species assemblages for all (All spp.), zooxanthellate $(\mathrm{Z})$ and azooxanthellate $(\mathrm{AZ})$ taxa between sites and habitats within the WMNP, Indonesia. $m$ is the maximum number of principle coordinate (PCO) axes with minimal misclassification; \% var. quantifies total variance explained by the first $m$ PCO axes; allocation success denotes the proportion of correct allocations to each group; $\delta^{2}$ is the first squared canonical correlation size.

\begin{tabular}{|c|c|c|c|c|c|c|c|c|c|}
\hline \multirow{2}{*}{$\begin{array}{l}\text { Factor } \\
\text { Site }\end{array}$} & \multirow[t]{2}{*}{$m$} & \multirow[t]{2}{*}{$\%$ var } & \multicolumn{5}{|c|}{ Allocation Success \% } & \multirow[t]{2}{*}{$\delta^{2}$} & \multirow[t]{2}{*}{$P$} \\
\hline & & & Sampela & Buoy 3 & Pak Kasim's & Ridge 1 & Total & & \\
\hline All spp. & 27 & 97.86 & 83.33 & 83.33 & 83.33 & 91.67 & 85.42 & 0.979 & 0.0001 \\
\hline Z & 10 & 93.97 & 83.33 & 75 & 83.33 & 91.67 & 83.33 & 0.883 & 0.0001 \\
\hline AZ & 28 & 99.53 & 100 & 66.67 & 50 & 100 & 79.17 & 0.991 & 0.0001 \\
\hline Habitat & & & Flat & Crest & Slope & & & & \\
\hline All spp. & 17 & 89.55 & 87.5 & 68.75 & 81.25 & - & 79.17 & 0.946 & 0.0001 \\
\hline Z & 5 & 82.24 & 87.5 & 56.25 & 68.75 & - & 70.83 & 0.676 & 0.0001 \\
\hline AZ & 8 & 71.86 & 100 & 43.75 & 62.5 & - & 68.75 & 0.516 & 0.0009 \\
\hline
\end{tabular}

Several azooxanthellate species within 5 families principally defined the reef slope (Figure 2.6). Two azooxanthellate colonies (Astrogorgia sp.19, Melithaea ochracea Linnaeus 1758) were encountered during the survey at Sampela, 70 species at Buoy 3, 94 species at Pak Kasim's and 161 species at Ridge 1 (Table 2.2). Species richness and diversity, with the exception of Sampela, were similar to the first model (Figure 2.2) for the crest and slope (Figure 2.4b, d, f, h). It is notable that the pattern of Acanthogorgia sp.5 on the reef crest at Buoy 3 (Figure 2.6b, d, Table 2.6), was replicated by Acabaria sp.23 at Ridge 1, also inhabiting the ceilings of caves, overhangs and crevices. Both species are as yet undescribed. Melithaea sp.3 showed distinct assemblages on the ridge top at Ridge 1 (Figure 2.6d). However, the vast majority of azooxanthellate taxa inhabited the reef slope with similar assemblage composition and distribution patterns across Buoy 3 and Pak Kasim's as evident by the reduced allocation success (an indicator of reduced site and habitat distinction; Table 2.3) and site $\mathrm{x}$ habitat interaction. 
Table 2.4. SIMPER analysis results indicating which gorgonian species (zooxanthellate and azooxanthellate) contributed the greatest dissimilarities between sites and habitats. Results presented as the average abundance (AvAb1 \& 2), species average (AvD) and cumulative dissimilarity contribution (AvD Cum\%).

\begin{tabular}{|c|c|c|c|c|c|c|c|c|c|c|c|}
\hline $\begin{array}{l}\text { All Species } \\
\text { SITE }\end{array}$ & $\begin{array}{l}\text { Av. Group } \\
\text { Diss \% }\end{array}$ & AvAb1 & $\operatorname{AvAb2}$ & AvD & $\begin{array}{c}\text { AvD Cum } \\
\%\end{array}$ & All Species HABITAT & $\begin{array}{l}\text { Av. Group } \\
\text { Diss \% }\end{array}$ & AvAb1 & AvAb2 & AvD & $\begin{array}{c}\text { AvD } \\
\text { Cum \% }\end{array}$ \\
\hline Sampela \& Buoy 3 & 88.95 & Sampela & Buoy 3 & & & Flat \& Crest & 86.27 & Flat & Crest & & \\
\hline Isis hippuris[LT] & & 0.4 & 0.25 & 5.79 & 6.51 & Isis hippuris[LT] & & 0.53 & 0.33 & 6.33 & 7.34 \\
\hline Acanthogorgia sp.5 & & 0 & 0.61 & 5.55 & 12.76 & Acanthogorgia sp.5 & & 0 & 0.44 & 4.2 & 12.21 \\
\hline Isis hippuris[L] & & 0.25 & 0.06 & 4.49 & 17.8 & Isis hippuris[L] & & 0.19 & 0.22 & 3.7 & 16.5 \\
\hline Isis hippuris[N] & & 0.5 & 0 & 4.03 & 22.33 & Isis hippuris[N] & & 1.29 & 1.16 & 3.65 & 20.73 \\
\hline Sampela \& Pak Kasim's & 93.71 & Sampela & Pak Kasim's & & & Flat \& Slope & 96.14 & Flat & Slope & & \\
\hline Isis hippuris[N] & & 0.12 & 1.81 & 9.58 & 10.23 & Isis hippuris [LT] & & 0.53 & 0 & 6.8 & 7.07 \\
\hline Briareum excavatum & & 0 & 0.67 & 5.13 & 15.7 & Isis hippuris [L] & & 0.22 & 0.01 & 3.86 & 11.09 \\
\hline Isis hippuris[LT] & & 1.16 & 0.02 & 4.33 & 20.32 & Isis hippuris[N] & & 1.29 & 0.04 & 3.61 & 14.84 \\
\hline Isis hippuris[L] & & 0.79 & 0.25 & 3.34 & 23.88 & Briareum excavatum & & 0.04 & 0.86 & 3.18 & 18.15 \\
\hline Sampela \& Ridge & 97.73 & Sampela & Ridge & & & Crest \& Slope & 84.88 & Crest & Slope & & \\
\hline Briareum stechei & & 0 & 2.98 & 7.05 & 7.21 & Isis hippuris [L] & & 0.22 & 0.12 & 3.83 & 4.51 \\
\hline Isis hippuris[N] & & 0.12 & 1.14 & 3.53 & 10.82 & Isis hippuris[N] & & 0.89 & 0.01 & 3.09 & 8.16 \\
\hline Menella lenzi & & 0 & 0.92 & 2.4 & 13.28 & Briareum excavatum & & 0.06 & 0.06 & 2.7 & 11.33 \\
\hline Isis hippuris[LT] & & 0.4 & 0.38 & 2.35 & 15.68 & Briareum stechei & & 0.28 & 0.86 & 2.66 & 14.47 \\
\hline Buoy 3 \& Pak Kasim's & 84.26 & Buoy 3 & Pak Kasim's & & & & & & & & \\
\hline Isis hippuris[N] & & 0.25 & 1.81 & 8.01 & 9.51 & & & & & & \\
\hline Briareum excavatum & & 0.08 & 0.67 & 4.81 & 15.22 & & & & & & \\
\hline Isis hippuris[L] & & 0 & 1.16 & 3.71 & 19.63 & & & & & & \\
\hline Acanthogorgia sp.4 & & 0.06 & 0.79 & 2.97 & 23.15 & & & & & & \\
\hline Ridge \& Buoy 3 & 89.19 & Buoy 3 & Ridge & & & & & & & & \\
\hline Briareum stechei & & 0.03 & 2.98 & 6.22 & 6.98 & & & & & & \\
\hline Isis hippuris[N] & & 0.25 & 1.14 & 2.91 & 10.24 & & & & & & \\
\hline Menella lenzi & & 0.08 & 0.92 & 2.13 & 12.63 & & & & & & \\
\hline Astrogorgia sp.4 & & 0.14 & 0.43 & 1.96 & 14.82 & & & & & & \\
\hline Ridge \& Pak Kasim's & 81.3 & Pak Kasim's & Ridge & & & & & & & & \\
\hline Briareum stechei & & 0.16 & 2.98 & 4.55 & 5.6 & & & & & & \\
\hline Isis hippuris[N] & & 1.81 & 1.14 & 2.95 & 9.23 & & & & & & \\
\hline Briareum excavatum & & 1.16 & 0.85 & 1.81 & 11.45 & & & & & & \\
\hline Menella lenzi & & 0.25 & 0.92 & 1.69 & 13.53 & & & & & & \\
\hline
\end{tabular}


Table 2.5. SIMPER analysis results indicating which zooxanthellate gorgonian species contributed the greatest dissimilarities between sites and habitats. Results presented as the average abundance (AvAb1 \& 2), species average (AvD) and cumulative dissimilarity contribution (AvD Cum\%).

\begin{tabular}{|c|c|c|c|c|c|c|c|c|c|c|c|}
\hline $\begin{array}{l}\text { Zooxanthellate } \\
\text { SITE }\end{array}$ & $\begin{array}{l}\text { Av. Group } \\
\text { Diss \% }\end{array}$ & AvAb1 & AvAb2 & AvD & $\begin{array}{c}\text { AvD } \\
\text { Cum \% }\end{array}$ & $\begin{array}{l}\text { Zooxanthellate } \\
\text { HABITAT }\end{array}$ & $\begin{array}{l}\text { Av. Group } \\
\text { Diss \% }\end{array}$ & AvAb1 & AvAb2 & AvD & $\begin{array}{c}\text { AvD } \\
\text { Cum \% }\end{array}$ \\
\hline Sampela \& Buoy 3 & 81.37 & Sampela & Buoy 3 & & & Flat \& Crest & 71.23 & Flat & Crest & & \\
\hline Isis hippuris[L] & & 0.42 & 0.06 & 16.26 & 19.98 & Isis hippuris[LT] & & 0.54 & 0.38 & 12.77 & 17.93 \\
\hline Briareum excavatum & & 0.06 & 0.4 & 11.78 & 34.46 & Isis hippuris[N] & & 1.32 & 1.22 & 10.71 & 32.96 \\
\hline Isis hippuris[LT] & & 0.5 & 0.24 & 10.2 & 47 & Isis hippuris[L] & & 0.28 & 1.01 & 8.46 & 44.83 \\
\hline Briareum stechei & & 0.06 & 0.34 & 9.54 & 58.73 & Briareum excavatum & & 0.69 & 0.1 & 7.89 & 55.91 \\
\hline Sampela \& Pak Kasim's & 84.66 & Sampela & Pak Kasim's & & & Flat \& Slope & 87.31 & Flat & Slope & & \\
\hline Isis hippuris[N] & & 0.16 & 1.89 & 18.57 & 21.93 & Isis hippuris [LT] & & 0.54 & 0 & 14.55 & 16.67 \\
\hline Briareum excavatum & & 0.06 & 0.6 & 13.97 & 38.43 & Isis hippuris[N] & & 1.32 & 0.05 & 13.2 & 31.79 \\
\hline Isis hippuris[L] & & 0.01 & 0.98 & 13.43 & 54.29 & Briareum excavatum & & 0.04 & 0.86 & 10.24 & 43.51 \\
\hline Isis hippuris[LT] & & 0.42 & 0.91 & 8.84 & 64.73 & Briareum stechei & & 0.69 & 0.01 & 9.25 & 54.1 \\
\hline Sampela \& Ridge & 91.19 & Sampela & Ridge & & & Crest \& Slope & 82.8 & Crest & Slope & & \\
\hline Briareum stechei & & 0 & 3.09 & 29.51 & 32.36 & Briareum excavatum & & 0.28 & 0.86 & 14.88 & 17.97 \\
\hline Isis hippuris[N] & & 0.16 & 1.15 & 10.47 & 43.84 & Isis hippuris[N] & & 1.22 & 0.05 & 14.65 & 35.66 \\
\hline Briareum excavatum & & 0.01 & 0.91 & 8.26 & 52.9 & Isis hippuris [L] & & 1.01 & 0.05 & 13.18 & 51.58 \\
\hline Isis hippuris[LT] & & 0.5 & 0.37 & 7.17 & 60.76 & Briareum stechei & & 0.17 & 0.47 & 8.77 & 62.18 \\
\hline Buoy 3 \& Pak Kasim's & 77.58 & Buoy 3 & Pak Kasim's & & & & & & & & \\
\hline Isis hippuris[N] & & 0.27 & 1.89 & 17.56 & 22.64 & & & & & & \\
\hline Briareum excavatum & & 0.03 & 0.98 & 13.13 & 39.56 & & & & & & \\
\hline Isis hippuris[L] & & 0.4 & 0.6 & 11.87 & 54.86 & & & & & & \\
\hline Briareum stechei & & 0.34 & 0.91 & 9.3 & 66.85 & & & & & & \\
\hline Ridge \& Buoy 3 & 85.38 & Buoy 3 & Ridge & & & & & & & & \\
\hline Briareum stechei & & 0.03 & 3.09 & 27.92 & 32.7 & & & & & & \\
\hline Isis hippuris[N] & & 0.27 & 1.15 & 9.25 & 43.53 & & & & & & \\
\hline Briareum excavatum & & 0.03 & 0.91 & 7.73 & 52.58 & & & & & & \\
\hline Isis hippuris[L] & & 0.4 & 0.5 & 6.6 & 60.31 & & & & & & \\
\hline Ridge \& Pak Kasim's & 71.56 & Pak Kasim's & 's Ridge & & & & & & & & \\
\hline Briareum stechei & & 0.16 & 3.09 & 20.11 & 28.11 & & & & & & \\
\hline Isis hippuris[N] & & 1.89 & 1.15 & 8.98 & 40.66 & & & & & & \\
\hline Briareum excavatum & & 0.98 & 0.91 & 8.56 & 52.62 & & & & & & \\
\hline Isis hippuris[L] & & 0.91 & 0.4 & 4.93 & 59.52 & & & & & & \\
\hline
\end{tabular}


Table 2.6. SIMPER analysis results indicating which azooxanthellate gorgonian species contributed the greatest dissimilarities between sites and habitats. Results presented as the average abundance (AvAb1 \& 2), species average (AvD) and cumulative dissimilarity contribution (AvD Cum\%).

\begin{tabular}{|c|c|c|c|c|c|c|c|c|c|c|c|}
\hline $\begin{array}{l}\text { Azooxanthellate } \\
\text { SITE }\end{array}$ & $\begin{array}{l}\text { Av. Group } \\
\text { Diss \% }\end{array}$ & AvAb1 & AvAb2 & AvD & $\begin{array}{c}\text { AvD } \\
\text { Cum \% }\end{array}$ & $\begin{array}{l}\text { Azooxanthellate } \\
\text { HABITAT }\end{array}$ & $\begin{array}{l}\text { Av. Group } \\
\text { Diss \% }\end{array}$ & AvAb1 & AvAb2 & AvD & $\begin{array}{c}\text { AvD } \\
\text { Cum \% }\end{array}$ \\
\hline Sampela \& Buoy 3 & 100 & Sampela & Buoy 3 & & & Flat \& Crest & 97.66 & Flat & Crest & & \\
\hline Acanthogorgia sp.5 & & 0 & 0.61 & 12.88 & 12.88 & Acanthogorgia sp.5 & & 0 & 0.44 & 8.8 & 9.01 \\
\hline Astrogorgia sp.4 & & 0.08 & 0 & 11.11 & 23.99 & Astrogorgia sp.6 & & 0.06 & 0 & 7.69 & 16.89 \\
\hline Acanthogorgia sp.4 & & 0 & 0.25 & 5.75 & 29.74 & Acanthogorgia sp.4 & & 0 & 0.69 & 6.3 & 23.33 \\
\hline Acabaria sp.23 & & 0 & 0.25 & 4.74 & 34.48 & Bebryce cf. indica & & 0.32 & 0.14 & 3.1 & 26.51 \\
\hline Sampela \& Pak Kasim's & 100 & Sampela & Pak Kasim's & & & Flat \& Slope & 97.4 & Flat & Slope & & \\
\hline Astrogorgia sp.4 & & 0 & 0.51 & 9.75 & 9.75 & Astrogorgia sp.6 & & 0.06 & 0 & 6.36 & 6.5 \\
\hline Ellisella ceratophyta & & 0 & 0.17 & 8.77 & 18.52 & Melithaea sp.3 & & 0 & 0.06 & 6.36 & 13.07 \\
\hline Acanthogorgia sp.4 & & 0.08 & 0 & 7.86 & 26.38 & Acanthogorgia sp.4 & & 0 & 0.81 & 2.83 & 15.97 \\
\hline Astrogorgia sp.6 & & 0 & 1 & 4.99 & 31.38 & Annella sp.1 & & 0 & 0.56 & 2.58 & 18.62 \\
\hline Sampela \& Ridge & 100 & Sampela & Ridge & & & Crest \& Slope & 86.38 & Crest & Slope & & \\
\hline Melithaea sp.3 & & 0 & 0.25 & 4.32 & 4.32 & Melithaea sp.3 & & 0 & 0.06 & 7.69 & 8.9 \\
\hline Menella lenzi & & 0 & 0.92 & 3.74 & 8.06 & Acanthogorgia sp.5 & & 0.44 & 0.08 & 2.24 & 11.5 \\
\hline Astrogorgia sp.4 & & 0 & 0.43 & 3.3 & 11.36 & Annella sp.1 & & 0.25 & 0.63 & 2.24 & 14.09 \\
\hline Astrogorgia sp.6 & & 0 & 0.38 & 3.16 & 14.52 & Acanthogorgia sp.4 & & 0.69 & 0.81 & 2.23 & 16.67 \\
\hline Buoy 3 \& Pak Kasim's & 92.46 & Buoy 3 & Pak Kasim's & & & & & & & & \\
\hline Astrogorgia sp.4 & & 0.31 & 0.51 & 11.75 & 12.7 & & & & & & \\
\hline Ellisella ceratophyta & & 0 & 0.17 & 10.25 & 23.79 & & & & & & \\
\hline Acanthogorgia sp.5 & & 0.61 & 0.05 & 3.67 & 27.76 & & & & & & \\
\hline Acanthogorgia sp.4 & & 0.25 & 1 & 3.36 & 31.39 & & & & & & \\
\hline Ridge \& Buoy 3 & 92.72 & Ridge & Buoy 3 & & & & & & & & \\
\hline Melithaea sp.3 & & 0 & 0.25 & 4.56 & 4.92 & & & & & & \\
\hline Astrogorgia sp.4 & & 0.14 & 0.43 & 3.68 & 8.89 & & & & & & \\
\hline Menella lenzi & & 0.08 & 0.92 & 3.47 & 12.63 & & & & & & \\
\hline Astrogorgia sp.6 & & 0 & 0.38 & 3.25 & 16.13 & & & & & & \\
\hline Ridge \& Pak Kasim's & 88.52 & Pak Kasin & 's Ridge & & & & & & & & \\
\hline Melithaea sp.3 & & 0 & 0.25 & 4.15 & 4.69 & & & & & & \\
\hline Astrogorgia sp.4 & & 0.05 & 0.43 & 3.36 & 8.49 & & & & & & \\
\hline Menella lenzi & & 0.25 & 0.92 & 3.21 & 12.11 & & & & & & \\
\hline Astrogorgia sp.6 & & 0.32 & 0.38 & 3.19 & 15.71 & & & & & & \\
\hline
\end{tabular}




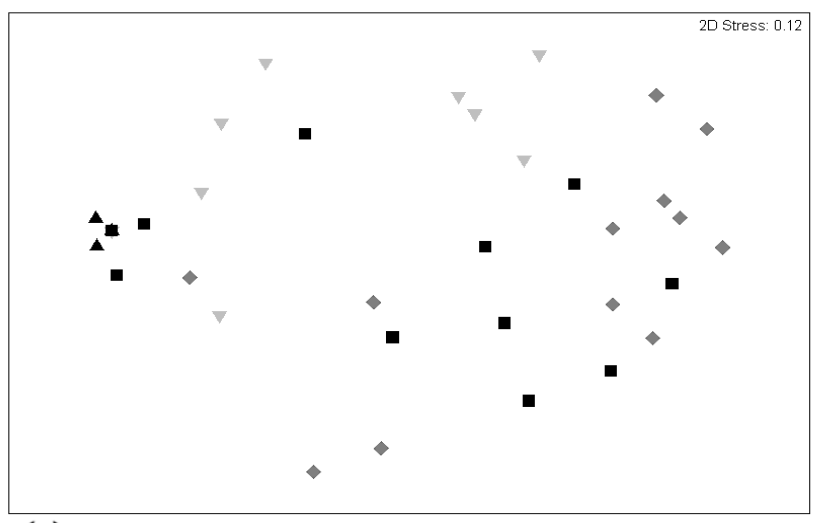

(a)

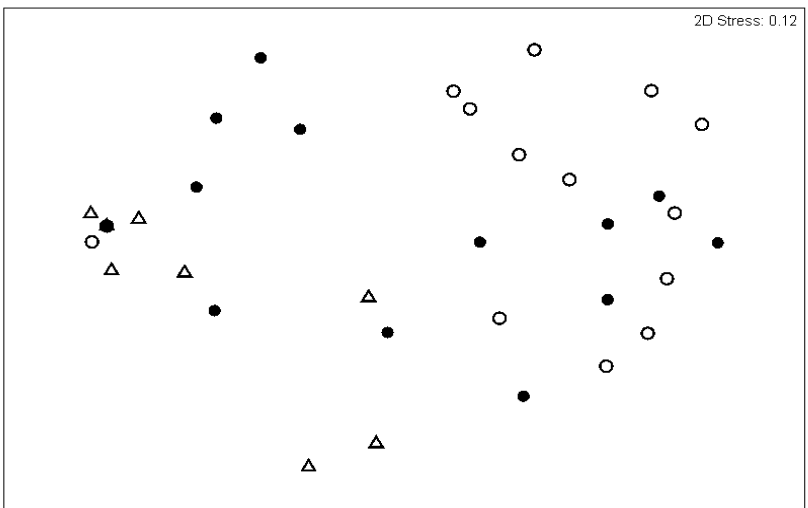

(c)
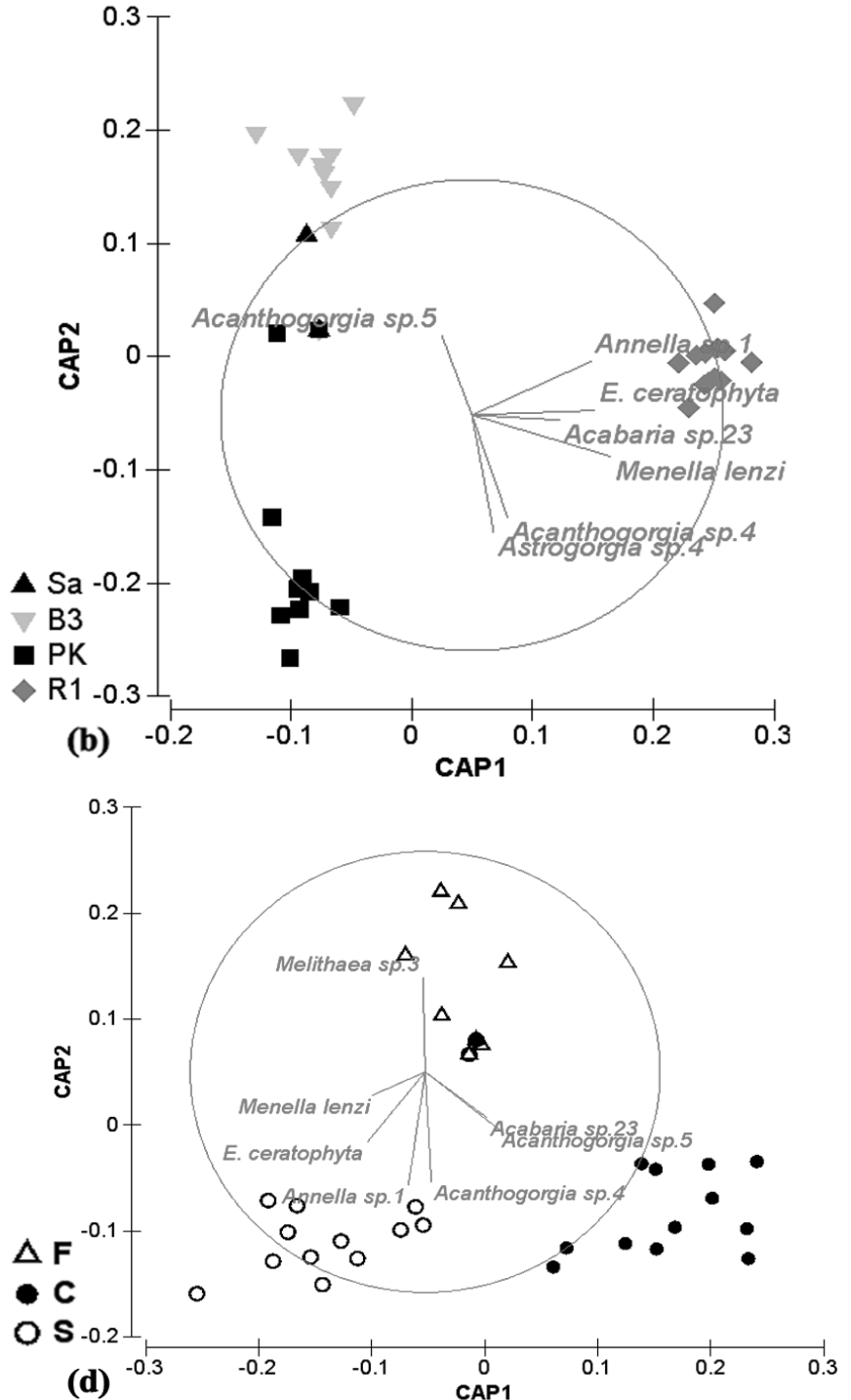

Figure 2.6. (a, c) Constrained and (b, d) unconstrained ordinations of azooxanthellate gorgonian assemblages between sites $(a, b)$ and habitats $(c, d)$. Species vectors are directed where the species were best represented. See Figure 2.3 for factor level codes.

Gorgonian Community Structure. Univariate and multivariate analyses illustrated clear differences between sites and habitats within the WMNP (Figures $2.2-2.7$ ). Seven families from the Calcaxonia (Ellisellidae $S=16$, Isididae $S \sim 2$ ), Holaxonia (Acanthogorgiidae $S=16$, Plexauridae $S=87$ ) and Scleraxonia (Briareidae $S=3$, Subergorgiidae $S=7$, and Melithaeidae $S=$ 44) characterized reef habitats from low diversity and abundance at the impacted site Sampela to high diversity and abundance at Ridge 1 (Figure 2.7).

The Isididae at Sampela were dominant across the flats and crest $\left(11.5 \pm 1 \& 11.5 \pm 1.4\right.$ per $20 \mathrm{~m}^{-2}$; mean $\pm \mathrm{SE}$ ), with occasional Briareidae on the slope (2 \pm 1$)$. At Buoy 3, Isididae were dominant on 


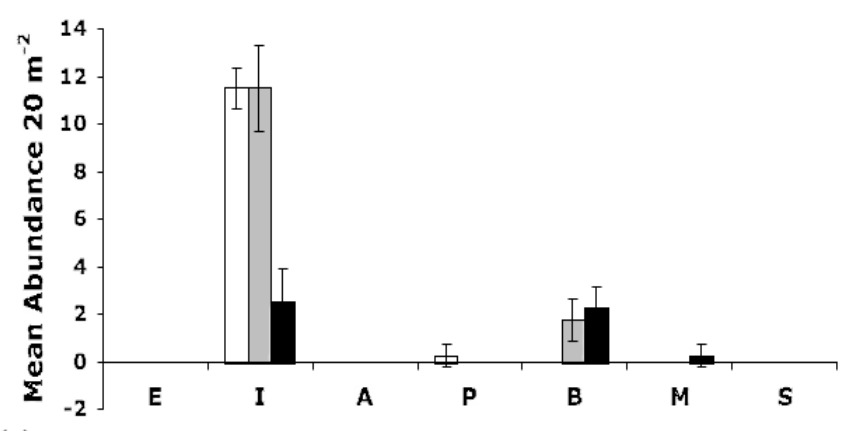

(a)

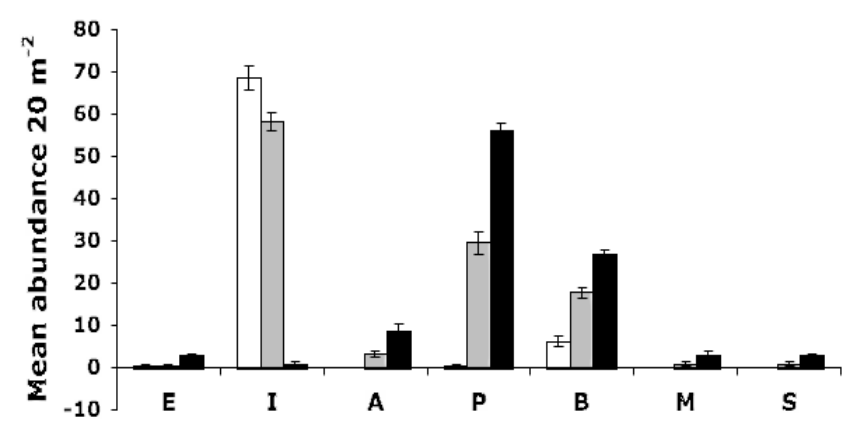

(c)

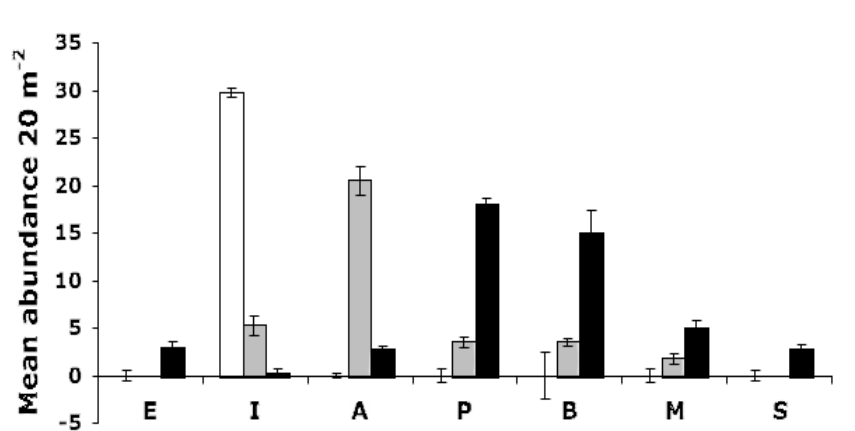

(b)

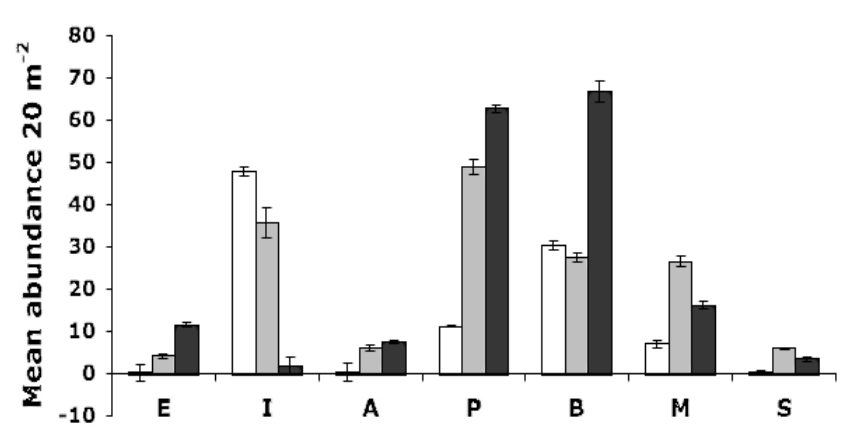

(d)

Figure 2.7. Site-specific gorgonian family abundance (mean $\pm \mathrm{SE}$ ) across sites and habitats within the WMNP. (a) Sampela, (b) Buoy 3, (c) Pak Kasim's, and (d) Ridge 1. E, Ellisellidae; I, Isididae; A, Acanthogorgiidae; P, Plexauridae; B, Briareidae; M, Melithaeidae; S, Subergorgiidae. White bars, reef flat; grey bars, reef crest; black bars, reef slope.

the reef flat $(30 \pm 2)$, Acanthogorgiidae characterised overhangs on the reef crest $(20 \pm 1.5)$, and Plexauridae on the reef slope (18 \pm 1$)$. Pak Kasim's was dominated by high numbers of the Isididae on the reef flat and crest $(68 \pm 3 ; 58 \pm 2)$, with Plexauridae and Briareidae on the reef slope (56 \pm 2 ; $27 \pm 1$ ). Ridge 1 showed Isididae and Briareidae having the greatest relative abundance on the ridge top ( $48 \pm 2 ; 30 \pm 3$ ), Plexauridae, Isididae, Briareidae and Melithaeidae on the reef crest ( $49 \pm 2$; $36 \pm 3 ; 28 \pm 1 ; 27 \pm 1$ ), and Plexauridae and Briareidae on the reef slope (63 $\pm 0.1 ; 67 \pm 1)$. In sum, phototrophic/zooxanthellate taxa added little diversity but greatest relative abundance to the reef flats and crest for Isididae and reef slope for Briareidae (Figure 2.7). Heterotrophic/azooxanthellate taxa especially within the family Plexauridae, contributed greatest to the increased biodiversity with depth (Figure 2.7).

Colony size was not reported in this study however numerous azooxanthellate species were small and located within sheltered crevices, overhangs, or at the base or under other coral colonies (e.g., the soft coral Sarcophyton Lesson 1834 and tabulate scleractinian Acropora Oken 1815). Interestingly, small colonies of Acanthogorgia sp.4, Annella sp.1 and Bebryce hicksoni Thomson \& Henderson 1905 were frequently encountered at the base of large Annella reticulata Ellis \& 

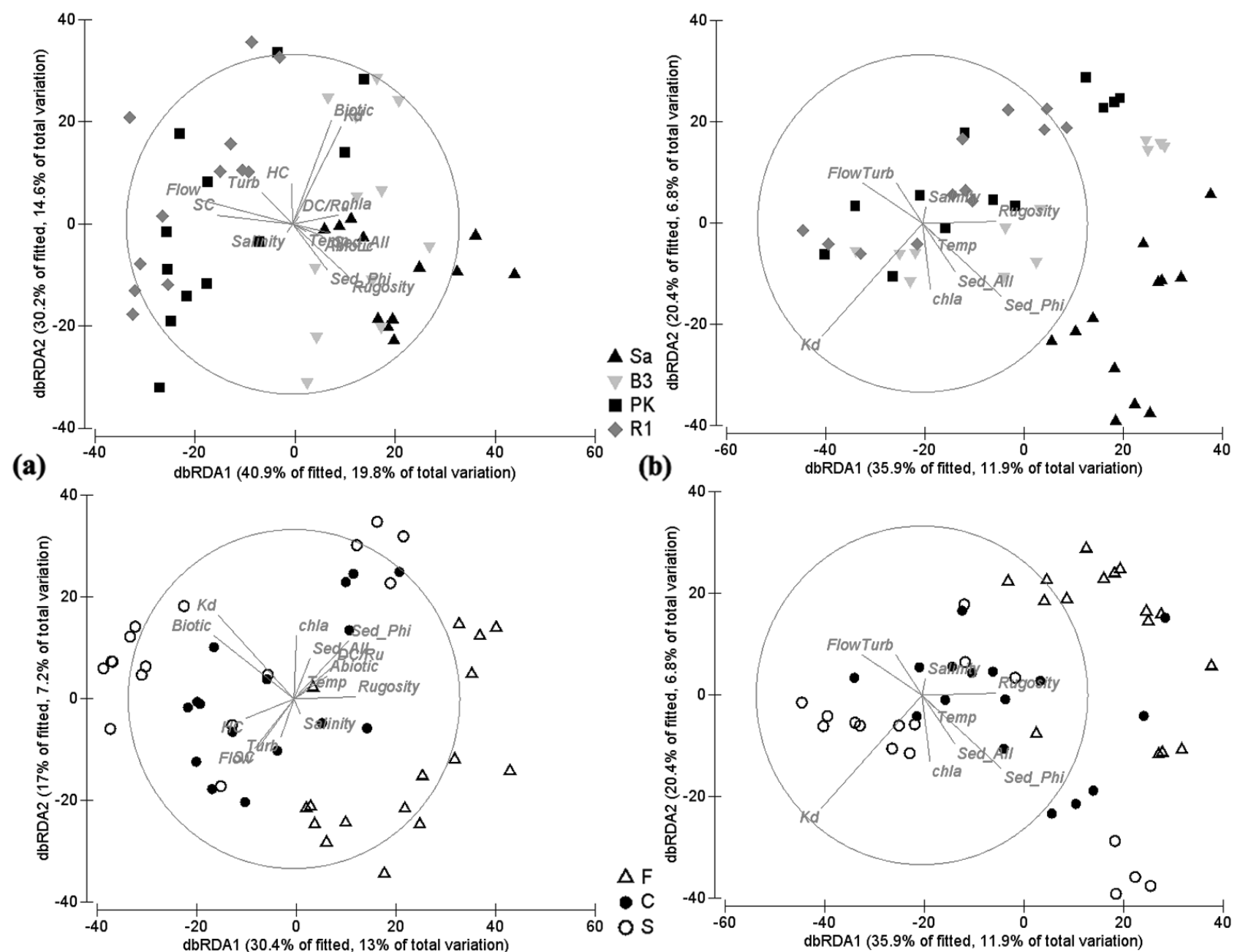

(c)

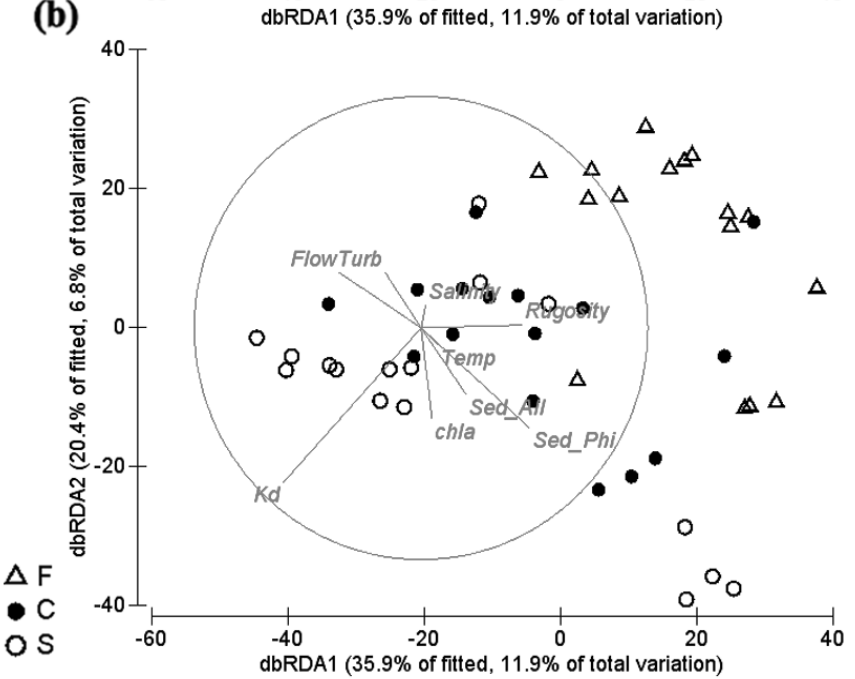

(d)

Figure 2.8. DISTLMforward models for the best explanatory drivers for variability across site (a, full variables; b, abated variables) and habitats (c, full variables; d, abated variables) of gorgonian variability across sites within the WMNP. See Figure 2.3 for factor level codes.

Solander 1736, A. mollis Nutting 1910, and Melithaea spp. colonies. Observations at depths greater than those reported here suggest a continual increase in gorgonian diversity, abundance and size, plus a remarkable frequency of new recruits $(<5 \mathrm{~cm}$ tall). Additional gorgonian species present within the WMNP not encountered during the surveys are documented in Table 2.2.

\subsubsection{Environmental Variables}

Results from the nonparametric multivariate regression DISTLMforward revealed that taken together biotic (sponges, algae, ascidians, molluscs) variables, grain size, rugosity, and light explained $23.73 \%$ (pseudo- $F=2.453, P<0.001$ ) of the variability in gorgonian assemblage structure (Figure 2.8). Benthic variables, covariates of gorgonian assemblages, were omitted from an additional analysis revealing sediment grain size, light, rugosity and chlorophyll- $a$ explaining $25.32 \%$ (pseudo- $F=1.999, P<0.001$ ) of gorgonian assemblage variability (Figure 2.8). The same 
model applied separately to zooxanthellate and azooxanthellate species revealed that soft coral, biotic variables and flow explained 29.3\% (pseudo- $F=3.601, P=0.001$ ), and flow, light and chlorophyll- $a$ explained $28.13 \%$ (pseudo- $F=3.904, P<0.001$ ) of the variability in both classes respectively. Results from the abated model suggested that water flow, light and chlorophyll- $a$ (27.6\%; pseudo- $F=3.134, P=0.003)$, rugosity, sediment grain size and light $(30.04 \%$; pseudo- $F=$ $6.254, P=0.001$ ) again had a limited, yet significant degree of influence on zooxanthellate and azooxanthellate distributions.

\subsection{DISCUSSION}

In total, 197 gorgonian species and distinct morphotypes from 42 genera and 12 families were documented across shallow $(0-30 \mathrm{~m})$ coral reefs within the WMNP, Indonesia. Comparable with previously described shallow water gorgonians across the Indo-Pacific comprising 51 genera within 14 families (Grasshoff 1999, Fabricius \& Alderslade 2001), this study strongly supports the WMNP as an area of high regional gorgonian abundance and diversity. Distinct community types across sites and habitats along an environmental gradient are characterised by non-independence between benthic space competitors, and driven in part by habitat complexity, water flow and natural light.

Gorgonian distribution patterns within the WMNP followed a gradient of low diversity and abundance at the impacted site Sampela to high diversity and abundance at Ridge 1. Species richness and diversity were proportional to depth, a pattern consistent with previous research on benthic invertebrates within the area (e.g., Porifera, Bell \& Smith 2004), yet the inverse for Scleractinia (Haapkylä et al. 2007). Similarly, high gorgonian diversity with depth has been shown in other areas (Singapore, Goh \& Chou 1994; Caribbean, Sanchez et al. 1997; Marianas, Paulay et al. 2003; Hong Kong, Fabricius \& McCory 2006; Japan, Matsumoto et al. 2007; Philippines, Rowley unpublished data) with concomitant zooxanthellate octocoral abundance in the shallows (Great Barrier Reef, Fabricius \& Klumpp 1995; Thailand, Chanmethakul et al. 2010).

Differences in gorgonian assemblage structure between sites and habitats were driven by morphotypes of the zooxanthellate isidid I. hippuris and species within the genus Briareum. I. hippuris dominance on the shallow reef flats is likely due, in part, to differential disturbance levels between study sites. Continual disturbance maintains relative species composition, stability and biodiversity within a particular reef community (Connell 1978, Aronson \& Precht 1995, Shea et al. 2004, Gouhier \& Guichard 2007, Bohn et al. 2014). For example, strong upwellings and water currents at Ridge 1, contrasting with continual resource exploitation and high sedimentation rates at Sampela, are reflected in diverse and impoverished gorgonian communities, respectively. Buoy 3 
and Pak Kasim's, both subject to past destructive fishing practices and bleaching on the former reef flats, show an increase in gorgonian abundance and diversity between Sampela and Ridge 1. However, considerable loose rubble and anthropogenic gleaning on the shallow ( 1-3 m) reef flats of Buoy 3 likely impede settlement success even at low turbulence (Goh \& Chou 1994), thus reflecting minimal recovery and gorgonian presence. Yet high I. hippuris abundance on the deeper ( 3-5 m) reef flats at Pak Kasim's may indicate that this species is an $r$-selected strategist, with its response to reef recovery unencumbered by inhibitors to settlement and growth, therefore, thriving in elevated current flow, low turbidity and minimal loose substrata.

The ability to colonize disturbed areas reflects reproductive strategy, larval settlement choices and post-settlement mortality. I. hippuris is a gonochoristic (Simpson 1906) external brooder (pers. obs.; see also Figure 4.5), yet also displays considerable fragmentation with a proportionally high regeneration capacity on the reef flats across all sites. Asexual propagation through fragmentation is not uncommon in gorgonians yet can show intra- and inter-disturbance sensitivity (Coffroth \& Lasker 1998). The proportional success of regenerative fragments, with most fragments being small and apical, across all sites suggests fragmentation in I. hippuris is not disturbance sensitive. Interestingly, such fragment success was morphotype-specific and may represent eco-phenotypic specificity or sibling taxa. Nevertheless, asexual propagation through fragmentation facilitates rapid post-disturbance recovery (Dauget 1992, Dahan \& Benyahu 1997) resulting in high local population abundance as evident by I. hippuris on the reef flats in the WMNP.

The zooxanthellate genus Briareum also influenced separations between the factors site and habitat. Low-lying branching species on the reef slope, predominantly at Pak Kasim's and Ridge 1, had a bathymetric distribution counter to that of both I. hippuris (this study) and scleractinians (Porter 1976). Furthermore, numerous asexual fragments and juvenile colonies were encountered. This pattern mirrored its Atlantic congener, Briareum asbestinum Pallas 1766, which reproduces through asexual fragmentation and external brooding producing low dispersal philopatric larvae (Brazeau \& Harvell 1994). It is likely that such a dual reproductive strategy occurs in Indo-Pacific Briareum taxa. However, reproductive strategies for Indo-Pacific gorgonian taxa are largely unknown, likely having limited dispersal abilities leading to habitat specialists and high endemism (Grasshoff \& Bargibant 2001, Picciano \& Ferrier-Pagès 2007).

Acanthogorgia sp.5 was the only azooxanthellate species driving differences between and within factor levels due to its exclusive abundance on the ceilings of caves and overhangs on the reef crest at Buoy 3. This specialized distribution may be due to within-overhang microhabitats, presettlement larval preferences such as negative phototaxis (Sánchez et al. 1997), geotaxis, or 
differential mortality following settlement in other areas. Species within the Acanthogorgia genus do however, possess a degree of habitat selectivity (Goh \& Chou 1994, this study), frequently observed at the base of other large chemically well defended gorgonians such as Annella reticulata, A. mollis, Melithaea spp. and the soft coral Sarcophyton. Such taxa likely affect recruitment (Yoshioka \& Yoshioka 1989, Yoshioka 1996) through waterborne exudates facilitating spatial refugia from predation, competition (Hay 1986) or fouling. Larval settlement preferences have been shown for crustose coralline algae (Harrington et al. 2004), in addition to enhanced photophysiological performance on carbonate substratum (Green et al. 2010). Furthermore, azooxanthellate Caribbean gorgonian larvae show settlement preference for consolidated topographically complex reefs in addition to longer pelagic larval duration (PLD; Sánchez et al. 1997) compared to zooxanthellate taxa. Yet both fitness enhancement through substratum selection and PLD are unknown for Indonesian gorgonians, PLD not necessarily predictive of dispersal (Rosen 1988, Weersing \& Toonen 2009, but see also Cowen et al. 2006). Nevertheless, diversity and abundance increased markedly with habitat complexity towards Ridge 1 and with depth. This bioenvironmental cline suggests higher larval availability through water currents and self-seeding, as well as selection and post-settlement success for sites with high topographic complexity and consolidated substratum. In contrast, low relief, unconsolidated fine-grain substratum coupled with low water flow, high sediment rate, continuous anthropogenic disturbance and high grazing activity from Diadema spp. at Sampela (Hodgson 2008) likely act in concert with reduced larval availability, settlement and survival to result in low biodiversity.

Habitat structural complexity, thus colonizable area, substratum type and light intensity, can determine settlement choices and profoundly influence benthic community structure on coral reefs (Sánchez et al. 1997, Meesters et al 2001, Linares et al. 2008). Yet the combination of predictor variables biotic, sediment grain size, rugosity and light explained only $23 \%$ of gorgonian assemblage structure across clear environmental clines. Evidently, two inherently related patterns were occurring. Firstly, benthic variables such as sponges, algae, hard and soft coral all co-vary with gorgonian distribution, themselves members of coral reef benthic communities. Remodeling on such covariate removal revealed sediment grain size, light, rugosity and chlorophyll- $a$ still explained only $25 \%$ of gorgonian assemblage structure. that, Secondly, this suggests that zooxanthellate and azooxanthellate gorgonian distribution are not independent of each other, essentially reflecting two different trophic groups, heterotrophs and phototrophs. This further confirms that interspecific competitive forces do exist between shallow water gorgonians relative to natural light as a function of bathymetry. 


\subsubsection{Zooxanthellate versus Azooxanthellate Gorgonians}

The dominance of zooxanthellate taxa driving separation between reef areas and location obscured azooxanthellate distribution patterns. Trophic group separation (zooxanthellate = phototrophy; azooxanthellate $=$ heterotrophy) revealed a clear environmental gradient interaction with depth, further confirmed by non-independence between the two groups. Thus both groups displayed contrasting patterns with azooxanthellate species richness and diversity proportional to depth, consistent with other areas (Goldberg 1973, Goh \& Chou 1994, Sanchez et al. 1997, Paulay et al. 2003, Fabricius \& McCory 2006, Matsumoto et al. 2007, this study), with the inverse being true for zooxanthellate taxa.

Replicate multivariate models for zooxanthellate and azooxanthellate taxa revealed zooxanthellate species drove differences between site and habitat. Distinct I. hippuris morphologies showed patterns of variability both within and between sites, most notably bushy colonies with long thick branches on the reef flat at Sampela and planar short tightly packed branched colonies at Ridge 1 . Morphological variants in 'sympatry' are not uncommon in Cnidaria (Knowlton 1993, Prada et al. 2008, Forsman et al. 2010) and may be indicative of phenotypic plasticity or incipient ecological divergence in response to natural light and water flow. Morphological variation in I. hippuris has previously been noted but not quantified (Simpson 1906, Fabricius \& Alderslade 2001). However such variation through increased branching surface area enhancing photosynthetic efficiency in shallow water branching taxa (Hennige et al. 2008), coupled with a previously undocumented dual mode of reproduction, may likely explain the biological success of I. hippuris across environmental clines within the WMNP. Colony form can depend on feeding strategy and the same genotype can show different allocation patterns in different environments (Weiner 2004). Yet different morphotypes within the same environment, may represent separate clonal aggregations (Coffroth \& Lasker 1998) consequential of fragmentation or the potential for sibling species.

Fitness enhancement through morphological plasticity relative to water flow and light availability may drive I. hippuris morphotype differences particularly between Ridge 1 and Sampela. Densely packed, shorter branches in high flow environments - coupled with high irradiance - at Ridge 1 maintains colony integrity and maximizes high particle capture, especially downstream of a colony or aggregation due to turbulence intensity (McFadden 1986, Sebens et al 1997). Conversely, long wide branches with a high surface area likely maximize photosynthetic gain (Hennige et al. 2008) otherwise absorbed by suspended particulate matter in high sedimented reefs (Anthony \& Fabricius 2000) such as Sampela, and further reducing sediment accumulation on the colony (Crabbe \& Smith 2003). Colony surface area and metabolism are intrinsically linked whereby variations in 
branching structure and concomitant within colony canalisation result in resource allocation plasticity, further representing alternate feeding strategies, size and growth patterns (Sebens 1997) to mitigate environmental change. Reciprocal transplant experimentation between Sampela and Ridge 1 assessing a suite of phenotypic traits, in addition to molecular analyses, may be fruitful in ascertaining species delineation or acclimatory capacity between I. hippuris morphotypes.

A dual reproductive strategy was also observed in Briareum spp. and likely explains, in part, the relative success of this species at depths where few zooxanthellate taxa are encountered and azooxanthellate diversity is high. Briareum morphotypes also displayed habitat specificity with branching taxa at depth and encrusting types on the high flow reef flat/Ridge top. Encrusting morphologies reduce drag in such high flow environments (Bell \& Barnes 2000, Bell \& Smith 2004). However, habitats characterised by low wave action, high turbidity and sedimentation rates, have also been shown to favour encrusting Briareum spp. (Fabricius \& Alderslade 2001, Fabricius \& De'ath 2004), likely due to morphological and behavioural pre-adaptations such as phenotypic and photoacclimatory plasticity, colony and polyp size, reproductive strategy and recruitment survival (Stafford-Smith 1993, Anthony 2000). Yet such patterns are in direct contrast with those in this study. Furthermore, Briareum spp. abundance at Sampela compared to I. hippuris was considerably less with three out of the seventeen colonies encountered being encrusting. Thus branching and lobe-like, upward projecting Briareum morphologies may well be selected for in low light and water flow, high turbidity and sedimented environments; reducing sediment smothering with increased SA:V for photosynthetic efficiency akin to I. hippuris.

Predictor variables highlight water flow, light and chlorophyll- $a$, rugosity, sediment grain size and light for zooxanthellate and azooxanthellate species respectively. High water motion and localized upwelling further enhanced by strong water currents at Ridge 1, fertilize the reef with deep benthic nutrients for primary productivity and enhanced food availability (Jokiel 1978, Sebens 1984), maximizing species biodiversity and abundance. Therefore, increased azooxanthellate species richness and diversity on the Ridge top, coupled with slightly reduced zooxanthellate species abundance compared to Pak Kasim's is indicative of a natural reef environment on the Ridge with overall reduced species dominance. Such patterns can again be attributed to intermediate disturbance levels, maintaining relative species diversity within a reef community, and thus acting in concert with nutrient and suitable substrate availability and competition (Connell 1978, Aronson \& Precht 1995, Townsend \& Scarsbrook 1997, Gouhier \& Guichard 2007).

Azooxanthellate gorgonian assemblage structure showed a relatively consistent pattern across sites and habitats with the exception of Sampela. However, an amplitudinal/additive interaction (i.e., not 
due to "crossing-over") revealed that proportionality of abundance between sites and habitats changed markedly for some taxa. Nevertheless, azooxanthellate gorgonians showed assemblage patterns consistent with an environmental decline from the healthy high energy Ridge to the depauperate reef communities at Sampela. Community structure of azooxanthellate taxa varied little within the deeper depths with only Plexauridae and Melithaeidae present across all sites. Species within the most diverse family, Plexauridae, drove diversity with depth, a pattern generally observed in other azooxanthellate families (Goh \& Chou 1994, Matsumoto et al. 2007, this study). Increased diversity and a high frequency of recruits with depth suggest a deeper refugia and competitor release from zooxanthellate corals. This pattern is similarly replicated by sponge taxa (Bell \& Smith 2004, Powell et al. 2010) inferring no or positive interactions between these two benthic groups (McLean \& Yoshioka 2007), both typically possessing powerful secondary metabolites. Moreover, increased azooxanthellate diversity with depth may likely represent a consistent biological source pool. Such taxa being invaluable in the event of past sea level variance in addition to current and future natural and anthropogenic disturbance particularly with regards the insidious effects of destructive fishing practices and global climate change.

Taken together, sedimentation, rugosity, light, and water flow have been shown to be major factors controlling local gorgonian populations (Sánchez et al. 1997, Meesters et al 2001, Linares et al. 2008). This pattern is true, in part, across environmental gradients within the WMNP. However, non-independence between zooxanthellate and azooxanthellate gorgonians and coral reef benthic variables likely explain the large amount of gorgonian assemblage variation unexplained by the predictor variable model. Niche partitioning through trophic differentiation (phototrophy and heterotrophy) is thus epitomized by gorgonian corals, greatly contributing towards coral reef biodiversity in the WMNP and undoubtedly the Coral Triangle as a whole.

\subsection{CONCLUSION}

In sum, gorgonian distribution patterns within the WMNP follow a gradient from low diversity and abundance at the impacted site at Sampela to high diversity and abundance at Ridge. Moreover, this environmental gradient response interacts with habitat primarily as a function of depth (thus light) structuring zooxanthellate and azooxanthellate taxa on shallow and slope reef habitats respectively. Light availability and benthic competitors define distribution and abundance for most gorgonian taxa. Most notable are morphological variants of the zooxanthellate species $I$. hippuris and species within the genus Briareum, such biological success likely being a consequence of dual reproductive strategies and morphological responses to different environments. However, whether such morphological variability, particularly in I. hippuris, is due to physiological capacity or fixed 
adaptations inferring incipient divergence due to differing resource allocation structure from prolonged exposure to sub-optimal environments, is unknown. Tests of physiological resilience of respective morphotypes would indeed be informative to management plans and coral reef biodiversity assessments. By determining species delineation and/or potential 'eco-morphotype' environmental specificity, monitoring of gorgonian taxa, in particular I. hippuris, can therefore greatly assist environmental impact assessments and identify areas of habitat degredation. 


\title{
CHAPTER 3: ENVIRONMENTAL INFLUENCES ON THE INDO-PACIFIC GORGONIAN ISIS HIPPURIS LINNAEUS 1758: PLASTICITY CAPACITY OR GENETICALLY FIXED?
}

\begin{abstract}
As conspicuous modular components of benthic marine habitats, gorgonian (sea fan) corals have perplexed taxonomists for centuries through their shear biodiversity, particularly throughout the Indo-Pacific. Phenotypic incongruence within and between seeming unitary lineages across contrasting environments can provide the raw material to investigate processes of disruptive selection. Two distinct phenotypes of the Isidid Isis hippuris Linnaeus 1758 partition across environmental clines: long-branched bushy colonies on degraded reefs, and short-branched multi/planar colonies on healthy reefs within the Wakatobi Marine National Park (WMNP), Indonesia. Multivariate analyses reveal phenotypic traits between morphotypes were likely integrated primarily at the colony level with increased polyp density and consistently smaller sclerite dimensions at the degraded site. Sediment load and turbidity, hence light availability, primarily influenced phenotypic division between the two sites. Thus the distinct morphological variability between the two sites is a reliable indicator of reef health; selection primarily acting on colony morphology, porosity through branching structure, as well as sclerite diversity and size. ITS2 sequence and predicted RNA secondary structure further revealed intraspecific variation between $I$. hippuris morphotypes relative to such environments $\left(\Phi_{\mathrm{ST}}=0.7683, P<0.001\right)$. This evidence suggests - but does not confirm - that I. hippuris morphotypes within the WMNP are two separate species, however to what extent and taxonomic assignment requires further investigation across its full geographic distribution. Incongruence between colonies present in the WMNP and tenuously described Isis alternatives (Isis reticulata Nutting 1910, Isis minorbrachyblasta Zou, Huang \& Wang 1991), questions the validity of such assignments. Furthermore, phylogenetic analyses corroborate early taxonomic suggestion that the characteristic jointed axis of the Isididae is in fact a convergent trait. Thus the polyphyletic nature of the Isididae lies in its type species $I$. hippuris, being unrelated to the rest of its family members.
\end{abstract}

Key words: Isis hippuris • Gorgonian coral • Isididae • Morphology・Indonesia • ITS2 


\subsection{INTRODUCTION}

Reef biodiversity reflects that of its environment, those within the Indo-Pacific Coral Triangle the most diverse of all. Intense competition in such environments leads to niche partitioning through resource acquisition, typically leading to coevolutionary divergence. Such diversification, the prerequisite for speciation, can occur with or without extrinsic barriers to gene flow and is particularly marked in sessile modular organisms such as cnidarians, far from passive to their environment (Cossins et al. 2006) in terms of growth form and chemical complement. However, delimitation between closely related species across steep environmental clines on coral reefs may be confounded by phenotypic plasticity, homoplasy, cryptic and sibling taxa (Knowlton 1993). It is, therefore, necessary to define environmentally driven divergent mechanisms on select phenotypic traits to accurately assess species biodiversity and endorse effective conservation management strategies (Taylor et al. 2006, Ladner \& Palumbi 2012).

Gorgonian corals (Cnidaria: Anthozoa: Octocorallia) are conspicuous modular organisms, the greatest diversity occurring within the Indo-Pacific coral triangle yet remarkably 'poorly known' (van Ofwegen 2004). Intra- and inter-specific morphological variability in gorgonians is influenced by environmental factors such as light, temperature, sedimentation and flow rates. However, little is known about the responses of gorgonian taxa to environmental parameters within the Coral Triangle. Distinct morphotypes of the isidid gorgonian Isis hippuris Linnaeus 1758 exist between healthy (Ridge 1) and degraded (Sampela) reefs within the Wakatobi Marine National Park (WMNP), SE Sulawesi, Indonesia; short-branched multi/planar colonies, and long-branched bushy colonies, respectively. Whether such morphological differentiation is a consequence of plasticity capacity, plasticity as an adaptation (Gotthard \& Nylin 1995, Hoogenboom et al. 2008), or genetically fixed leading to two species, is unclear.

Isis hippuris within the WMNP may be indicative of 'robust' canalisation where morphotypes are in fact two previously diverged species through disruptive (in sympatry) selection on traits between environments (Schluter, 1998, 2001, Campbell, 2003). Alternatively, physiological developmental constraints may have become decanalised through an acute perturbation or more likely accumulative cryptic genetic variation leading to evolutionary capacitance breakdown with subsequent fixation through genetic assimilation, itself a consequence of epigenetic heritability. In the first scenario, existence in low water velocity, high turbid environments typical of lagoon, semilagoon or sea-grass beds, as seen in Sampela, gave rise to an accumulation of pre- or postzygotic isolation between populations leading to separate adaptive fitness peaks representing ecological niches of long standing. Divergent morphotypes would therefore be robust to environmental 
change, maintaining native phenotypic traits. In the second scenario, cryptic variation more likely to be adaptive than random mutations, facilitate rapid mutation, as can acute perturbations (Flatt 2005). Either case can be accelerated by pleiotropy, linkage disequilibrium or concerted evolution (Sánchez \& Lasker 2003) leading to population level genetic assimilation of a particular phenotype and thus provides a testable level of emergent trait integration. Moreover, phenotypic variation can be largely attributed to rapid evolutionary events (Eldredge \& Gould 1972, Mattila \& Bokma 2008, Simpson 2013).

The phenomenon of species delimitation in closely related modular organisms can be investigated through models of integration (Magwene 2001). Growth persists through the iterative addition of modules (e.g., polyps, branching properties), which may develop independently or in concert (by trait integration, see Magwene 2001, Sánchez \& Lasker 2003, de Kroon et al. 2005, Sánchez et al. 2007) leading to differential integration in response to environmental perturbations. The co- and multi-variance of certain phenotypic traits may differ due to inextricably linked developmental (e.g., heterochrony; Sánchez 2004) or functional integration leading to patterns of diversity through plasticity or divergent selection directly (extrinsic) or indirectly (intrinsic) on traits between populations or subpopulations (Schluter, 2001, Turelli et al. 2001). By measuring five morphological traits in twenty-one Caribbean gorgonian species, Sánchez \& Lasker (2003) revealed integration within both branching and polyp dynamics yet were independent of each other. Furthermore, colony form and growth via branching were interconnected through the ratio of 'mother' branches to 'daughter' branches (Sánchez et al. 2004). Whether this is replicated across all gorgonians i.e. from different regions and habitats, is unclear, however species-specific trait integration particularly in response to environmental change has been shown in other taxonomic groups (e.g., plants; Xu et al. 2012).

Isis hippuris may simply possess high plasticity capacity, itself an adaptation facilitating considerable physiological tolerance to environmental heterogeneity, not uncommon in gorgonians (West et al. 1993, West 1997, Brazeau \& Harvell 1994, Kim et al. 1997, 2004, Skoufas 2006). Long-branched bushy, porous colonies reduce sediment settlement and maximise light capture through increased surface area and decreased self-shading in reduced light and water flow environments as seen in the scleractinian Stylophora pistilata Esper 1797 (Shaish et al. 2006). Whereas the densely packed short branches of planar colonies in high light and water flow, coupled with greater densities of small micro-skeletal elements (sclerites) provide mechanical strength (Grigg 1972, West et al. 1993, Kim et al. 2004, but see Skoufas 2006). Sclerites are key characters for species delineation within Octocorallia, those of the coenenchyme surface and polyps most 
susceptible to environmental variation (Bayer \& Stefani 1987). Reduced polyp density with depth as a function of light in zooxanthellate taxa (West et al. 1993, Kim et al. 2004, Prada et al. 2008, Prada \& Hellberg 2013) increases photosynthetic gain through surface area, yet polyp dimensions decouple integration by remaining independent of branching dynamics in Caribbean taxa (Sanchez \& Lasker 2003, Sanchez et al. 2004, 2007). A broad trait assessment including genetic analyses would therefore provide further insight into the relationship between I. hippuris and its environment, if such trait patterns are commensurate with those described in other gorgonians, and thus fixed within the phylogenetic group (e.g., Lasker \& Sánchez 2003, Sánchez 2004, Sánchez et al. 2004, 2007).

A single representative of its genus, I. hippuris has a recognised plasticity (Wright \& Studer 1889, Simpson 1906, Thomson \& Simpson 1909, Bayer \& Stefani 1987, Fabricius \& Alderslade 2001) and taxonomic uncertainty (Watling et al. 2012), which obscures any possible species boundaries. In order to fully elucidate the nature of I. hippuris phenotypic variation between reef sites, a brief taxonomic and historical account of the genus is presented with subsequent investigation into potential adherence to previously documented lower taxonomic assignments.

The family Isididae Lamouroux 1812 [nom. correct. Kükenthal 1915 (pro Isidae Lamouroux 1812)], itself currently within the sub-Order Calcaxonia, is characterised by a unique axis of alternating calcareous internodes and proteinaceous (gorgonin) nodes giving a bamboo appearance. Calcareous internodes can be hollow or solid and are not sclerobastic (i.e., consisting of fused sclerites sensu the sub-group Scleraxonia). The Isididae was further subdivided into four currently accepted subfamilies (see Bayer \& Stefani 1987, Alderslade 1998) primarily based on polyp retractability and sclerite composition and arrangement. Pertinent to this study, the sub-family Isidinae Lamouroux 1812 (sensu Studer 1887) is distinguished by small, warty and usually irregular sclerite forms and contains the genera Isis Linnaeus 1758 and Chelidonisis Studer 1890. Within the Isis genus 20 species have been assigned with currently only Isis hippuris Linnaeus 1758, the type species for the Isididae, Isidinae and genus Isis being widely accepted (Bayer \& Stefani 1987, Fabricius \& Alderslade 2001). I. reticulata Nutting 1910 and I. minorbrachyblasta Zou, Huang \& Wang 1991 have occasional reference yet with some taxonomic misgiving (see Bayer \& Stefani 1987, but also Mai-Bao-Thu \& Domantay 1971), therefore are briefly discussed to investigate divergent character traits similar to those found within the WMNP.

Originally (pre-Linnaen) Hippuris saxea Clusius 1605 (“the stony horse-tail"), Isis hippuris Linnaeus 1758 has for centuries been admired for its distinct articulated axis (Figure 3.1a) and thus named after the most important ancient Egyptian deity, the Goddess Isis. To this day the axis can 
be found in jewellery, art, souvenirs and even Royal collections; however, not until Ellis \& Solander (1786) was this taxon documented with its somewhat uninspiring mustard-brown outer soft tissue (coenenchyme: Figure 3.2). Nevertheless, conspicuous on coral reefs and in sea grass beds throughout the Central Indo-Pacific, I. hippuris contains potent secondary metabolites with anti-viral (Bordeleau et al. 2006, Chen et al. 2011) and anti-cancer (Susilaningsih et al. 2009) properties, likely contributing to its ecological success (Chapter 2). That said, correct taxonomic assignment using multiple lines of evidence across morphotypes within different geographical locations, are necessary given the clear ecological, pharmaceutical and inherent conservation importance of I. hippuris.

\author{
Sub-Class OCTOCORALLIA \\ Order ALCYONACEA Lamouroux 1812 \\ Sub-Order CALCAXONIA Grasshoff 1999 \\ Family ISIDIDAE Lamouroux 1812 \\ Sub-Family ISIDINAE Lamouroux 1812 \\ Isis hippuris Linnaeus 1758
}

(Figure 3.1a)

See Bayer \& Stefani 1987 for list of references [p. 55]

Type Material - Unfound, however 'authentic' specimens were collected and fully defined from Amboina, Indonesia (Milne-Edwards \& Haime 1857).

Diagnosis - These arborescent colonies can be up to $1 \mathrm{~m}$ tall, planar or bushy with lateral or partially dichotomous branching but rarely anastomosing (net-like). Branch formations may also give a candelabrum appearance. The axis consists of alternating calcareous internodes that reduces to a fine rod through the non-scleritic and convex, dark proteinaceous (gorgonin) nodes, the former typically longer than the latter. Branching is internodal in single or multiple planes, the latter giving rise to the bushy appearance. Branch lengths and diameters are variable, and up to three short branches can arise per internode with some so close they appear nodal in highly 


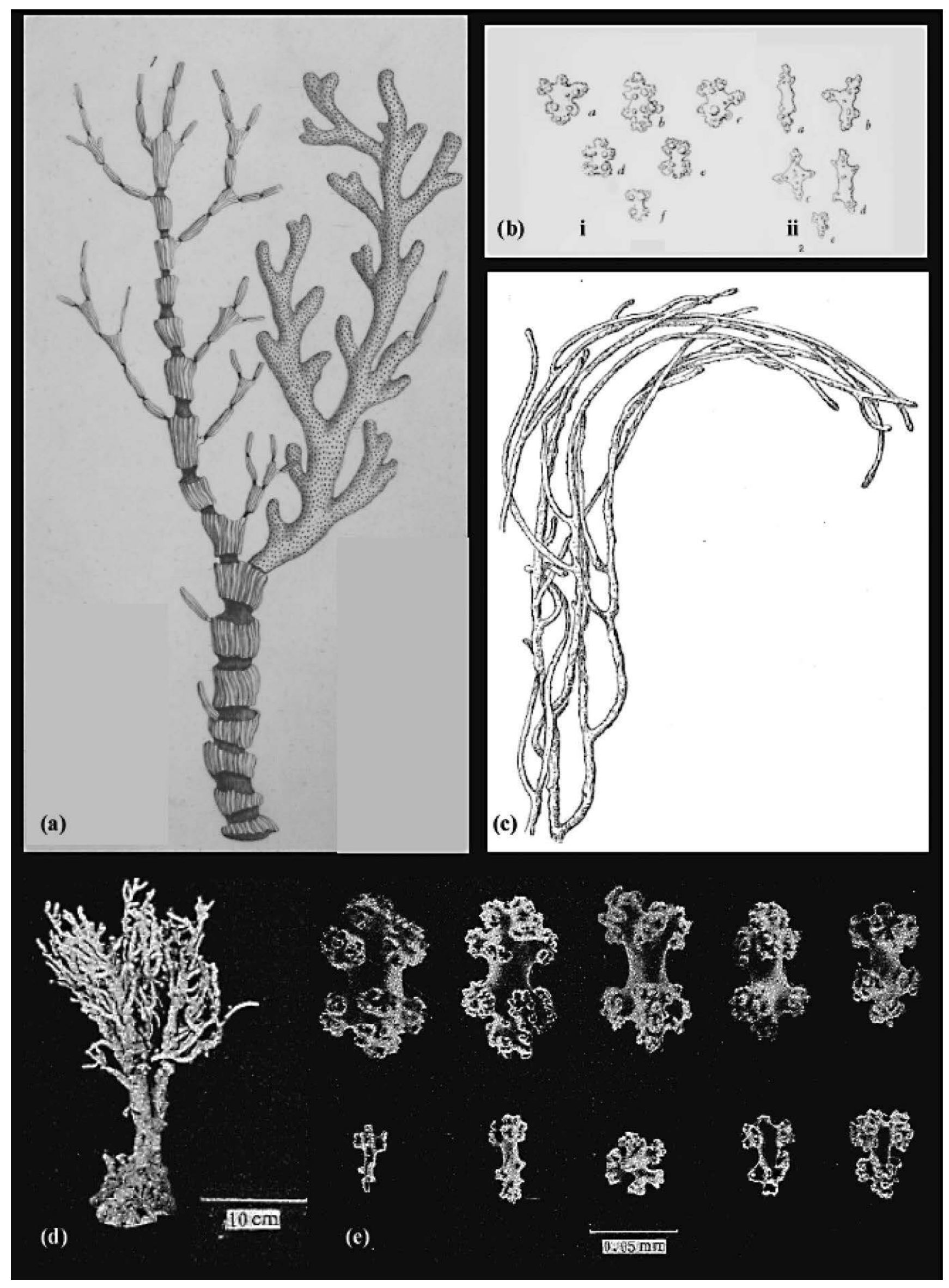


Figure 3.1. (Previous page) Isis Linnaeus 1758 comparisons of (a) Isis hippuris Linnaeus 1758 colony in Ellis \& Solander 1786; (b) sclerites of: i. I. hippuris and ii. Isis reticulata in Nutting 1910; (c) I. reticulata in Nutting 1910; (d) Isis minorbrachyblasta Zou et al. 1991 colony and (e) sclerites. Note, images sourced from each citation respectively.

branched colonies. The expanded calcareous cup-shaped base obliterates any trace of nodal composition particularly in older colonies. Calcareous internodes are sclerobastic (consisting of fused sclerites; but also see Nutting 1910c) with fibres arranged radially from a central core, akin to that of the sclerites bearing close resemblance to the Scleraxonia (Bayer 1955). Internodes possess longitudinal grooves corresponding with $8-12$ sinuous water vascular canals of $\sim 1 \mathrm{~mm}$ diameter. Polyps distributed all around the branches are $0.5-1.25 \mathrm{~mm}$ apart and fully retractile to $\sim 1.25 \mathrm{~mm}$ deep (and wide) into the thick coenenchyme. Such polyps bear eight lanceolate pinnate tentacles, and can possess up to three large (max. $1 \mathrm{~mm}$ diameter) round eggs, likely explaining the often swollen appearance of the branch tips as opposed to being a diagnostic feature (but see Nutting 1910c, Mai-Bao-Thu \& Domante 1971).

A diverse range of sclerites (Figure 3.1b, e) exists within the thick coenenchyme. The surface layer consists of small warty clubs $0.08 \times 0.001 \mathrm{~mm}$ [note: typographical error pg. $55,2^{\text {nd }}$ paragraph, Bayer \& Stefani 1987] typically bearing three large warts below the head wart (Figure 3.1b.i). Throughout the sub-surface layer some or all of the following are present in varying dimensions, asymmetry and commonly girdled: 6-, 7-, or 8-radiate capstans up to $0.19 \mathrm{~mm}$ in length, dumbbells/double heads (considered derivatives of 6-radiates; Bayer \& Stefani 1987) up to 0.32 $\mathrm{mm}$ long, warty or tuberculate spindles up to $0.25 \mathrm{~mm}$ long, and crosses. Very small e.g. rods of 0.07 x $0.01 \mathrm{~mm}$ (Bayer \& Stefani 1987), or no sclerites may be present within the polyp structures (Kölliker 1865). However smaller forms from those found within the coenenchyme (Simpson 1906, Thomson \& Simpson 1909, Kükenthal 1919, 1924) as well as small warty clubs with short handles $\sim 0.055 \times 0.045 \mathrm{~mm}$ located within the tentacles have been reported (Simpson 1906, Thomson \& Simpson 1909).

Sclerites colourless and colonies typically light brown to mustard yellow, with slightly darker polyps. 
Distribution - Central Indo-Pacific including Great Barrier Reef, Vanuatu, Papua New Guinea, Indonesia, Malaysia, Andaman, Philippines, Taiwan, Palau, South China Sea, Japan including Okinawa and the Ryukyu Islands.

Remarks - As evident from the description above, substantial phenotypic plasticity, from colony and branching structure to sclerite composition, exists in described specimens of I. hippuris (Wright \& Studer 1889, Simpson 1906, Thomson \& Simpson 1909, Bayer \& Stefani 1987, Fabricius \& Alderslade 2001). Whether such plasticity is a consequence of environmental influence within and between its distributions, or significantly structured to be more than one species is unclear. Thus, for such a ubiquitous and well-known species it "has been very imperfectly described" (Thomson \& Simpson 1909) leading to "a slender basis on which to raise a superstructure of classification" (Wright \& Studer 1889). Clearly a revision of the Isis genus is required including thorough analyses of specimens throughout its geographic range, as such character trait variability may be ecologically dependent (Bayer \& Stefani 1987). Attempts, however, have been made to differentiate phenotypic patterns within the Isis genus that, even though somewhat tenuous, may equate to the morphotypes found within the WMNP (Chapter 2). $I$. reticulata Nutting 1910 and I. minorbrachyblasta Zou, Huang \& Wang 1991 are therefore summarized below highlighting differences between the selected taxa.

\section{Isis reticulata Nutting 1910}

(Figure 3.1b)

See Mai-Bao-Thu \& Domantay (1971) for list of references [p. 28]

Type Material - Syntypes: several fragments of varying sizes, ZMA COEL. no. 2721, Siboga Expedition, station 149 or 273 at Pulu Jedan, Aru Islands, Maluku, Indonesia, 13 meters on sand and shells. Fragment donated to State University of Iowa (van Soest 1979). Specimens not located on request.

Diagnosis - Slender colonies, typically arborescent with long slim terminal branches that are not swollen at the ends. Few very small polyps irregularly distributed around the branches, the latter occasionally anastomosing. Sclerites of the coenenchyme bear sharp rough warts symmetrically distributed around delicate spindles and clubs the latter $0.04-0.06 \mathrm{~mm}$ in length. Some spindles curved possessing large tubercules. Irregular radiates $0.06 \times 0.03 \mathrm{~mm}$ to $0.2 \times 0.1 \mathrm{~mm}$ in length and 
width respectively, smooth warty rods $0.1-0.15 \mathrm{~mm}$ long and occasional crosses present. No polyp sclerites reported.

Sclerites colourless, colony reddish brown with slightly darker polyps in alcohol. Also noted as "brownish white" by Mai-Bao-Thu and Domantay (1971).

Distribution - I. reticulata has been documented in Indonesia (fragments from a single location 13 $\mathrm{m}$ depth), the Philippines ( 2 specimens and some fragments from a single location 12 - $15 \mathrm{~m}$ depth; Mai-Bao-Thu \& Domantay 1971), and Xisha Islands of China (single specimen and location; Zou, et al. 1991).

Remarks - I. reticulata is thus differentiated from I. hippuris on the basis of planar versus bushy colonies, long thin sinuous branches without swollen ends versus short thick antler-like branches with swollen ends, and all sclerites of a smaller size with sharp rough warts in I. reticulata. Sclerite differences between I. hippuris and I. reticulata have been considered questionable owing to the huge diversity in form (Stiasny 1940, Bayer \& Stefani 1987). However, Nutting (1910c) observed smaller and more sharply warted sclerites further corroborated by Kükenthal (1924) and Mai-BaoThu \& Domantay (1971), with illustrations showing marked asymmetry (Figure 3.1b.ii) contrary to that described. Curiously, Nutting (1910c) noted I. reticulata having flaccid polyps if preserved when extended due to their lack of sclerites. However, in I. hippuris, polyp sclerites were "not being evident on account of their small size" (Nutting 1910c) with no further discussion, lending question to their presence at all (Simpson 1906, Simpson \& Thomson 1909, Fabricius \& Alderslade 2001, but see Bayer \& Stefani 1987, Kölliker 1865). Conflicting sclerite images between Nutting (1910c) and Mai-Bao-Thu \& Domantay (1971), in addition to regional differences between specimens of I. hippuris (see Bayer \& Stefani 1987) having some adherence to I. reticulata, lends further question to its validity as a taxon. Finally, colony and polyp colouration may be an artifact of preservation; Nutting's 'pink' likely from buffered formalin used at that time (note: Rowley, S.J., examined a 'pink' specimen from the Siboga expedition at the British Natural History Museum [BNHM. 1889.6.28.18], which adhered closely to the I. hippuris description above and not the proposed I. reticulata), and Mai-Bao-Thu \& Domantay's 'white' from endosymbiotic bleaching not uncommon with, in particular, damaged Isis specimens (e.g., Thomson \& Simpson 1909). In summary, the distinction between I. hippuris and I. reticulata is conflicting and unclear, requiring further investigation. 
(Figure 3.1c)

Type Material - Holotype (G85-001) and paratype (G87-031) from two locations of the Nansha Islands, China.

Diagnosis - Colonies bushy with distal branches densely aggregated, themselves bearing tufts of branchlets no longer than $5 \mathrm{~cm}$ (ave. $3.5 \mathrm{~cm}$ ). The short, fine branches arise from the scleritic internodes. Tiny polyps are equally distributed around the branches. Coenenchyme sclerites up to $0.140 \times 0.091 \mathrm{~mm}$ being predominantly dumbbells and double heads with tubercules generally symmetrically arranged. Assortment of small clubs also present 0.06 x $0.025 \mathrm{~mm}$ with occasional crosses.

Sclerites colourless, colonies light brown in alcohol.

Distribution - Nansha Islands, China.

Remarks - Zou et al. (1991) state that the bushy non-planar colonies of I. minorbrachyblasta differ from the planar ones of I. hippuris and I. reticulata, in direct contrast to previous reports (e.g., Nutting 1910, Mai-Bao-Thu \& Domantay 1971). Furthermore, the branches for $I$. minorbrachyblasta are fine, short and densely packed, whereby I. hippuris and I. reticulata are thick, short, dense, and fine, long, anastomosing and loosely packed respectively; thus $I$. minorbrachyblasta an intermediate between the two. Statistical significance between select morphological traits (branchlet and sclerite length and width) revealed differences among taxa were between I. minorbrachyblasta and I. reticulata. However, it is unclear what sclerites were used for comparative analyses, and $\mathrm{n}=1$ in all cases. Based on the information presented here, any appreciable difference in colony, branch and sclerite composition especially between $I$. minorbrachyblasta and I. hippuris (e.g. Nutting 1910, Bayer \& Stefani 1987) is nebulous. Finally, Zou et al. (1991) propose I. minorbrachyblasta based on one or two specimens per taxon, somewhat unsatisfactory given both the nature of Isis phenotypic variability and analyses taken from a single region.

Isis within the Wakatobi - Isis morphotypes found within the WMNP bear only partial adherence to those described above at the colony level. The long-branched bushy colonies on degraded reefs, and short-branched multi/planar colonies on healthy reefs may reflect I. reticulata and I. hippuris respectively, or simply the widely accepted plasticity of the latter (Wright \& Studer 1889, Simpson 
1906, Thomson \& Simpson 1909, Bayer \& Stefani 1987, Fabricius \& Alderslade 2001), likely through an integration effect (Magwene 2001, Sanchez \& Lasker 2003, de Kroon et al. 2005).

Clearly Isis taxonomy is in a state of flux, compromising conservation efforts due to difficulties in species assignment. Here an assessment of morphotypes found within the WMNP relative to reef health is presented, with brief comparisons to those previously described. A thorough and necessary examination of Isis specimens throughout its distribution is however, underway and outside of the scope of this study. Given the tenuous nature of previously described Isis species bar I. hippuris, from here on in all specimens will remain assigned to I. hippuris, unless otherwise specified.

Research Question - Are populations of I. hippuris morphotypes phenotypically and genetically subdivided relative to environmental gradients within the WMNP, Indonesia? Do the I. hippuris morphotypes represent previously described species, or a single species with highly variant, integrated phenotypic traits?

This study therefore, aims to: (1) investigate morphological variability in the zooxanthellate gorgonian I. hippuris across environmental gradients on coral reefs within the WMNP, SE Sulawesi, Indonesia; (2) identify patterns of genetic variability relative to such morphotypes using population genetics and predicted RNA secondary structure of the nuclear ribosomal ITS2 region, (3) to subsequently infer mechanisms of speciation or phenotypic plasticity as a consequence of environmental change, and (4) investigate the currently assigned phylogenetic position of $I$. hippuris within the Octocorallia using the ITS2 region.

\subsection{METHODS}

\subsubsection{Study Area}

The Wakatobi Marine National Park (WMNP) is a remote archipelago of ca. $13,900 \mathrm{~km}^{2}$ in S.E. Sulawesi, Indonesia (Figure 2.1a). The epicentre of the Coral Triangle and Indonesia's second largest marine park, the WMNP comprises ca. $600 \mathrm{~km}^{2}$ of the most biodiverse coral reefs on earth (Scaps \& Denis 2007). Such marine biodiversity sustains $>100,000$ people with alarming human population expansion and consequential marine resource dependence and destructive commercial fisheries (Clifton et al. 2010, Clifton 2013). Coral reefs within the Wakatobi range from low current, high turbidity lagoons to highly exposed sites with strong water currents and high nutrient deep-water upwellings. Therefore, strong environmental gradients of natural and anthropogenic 


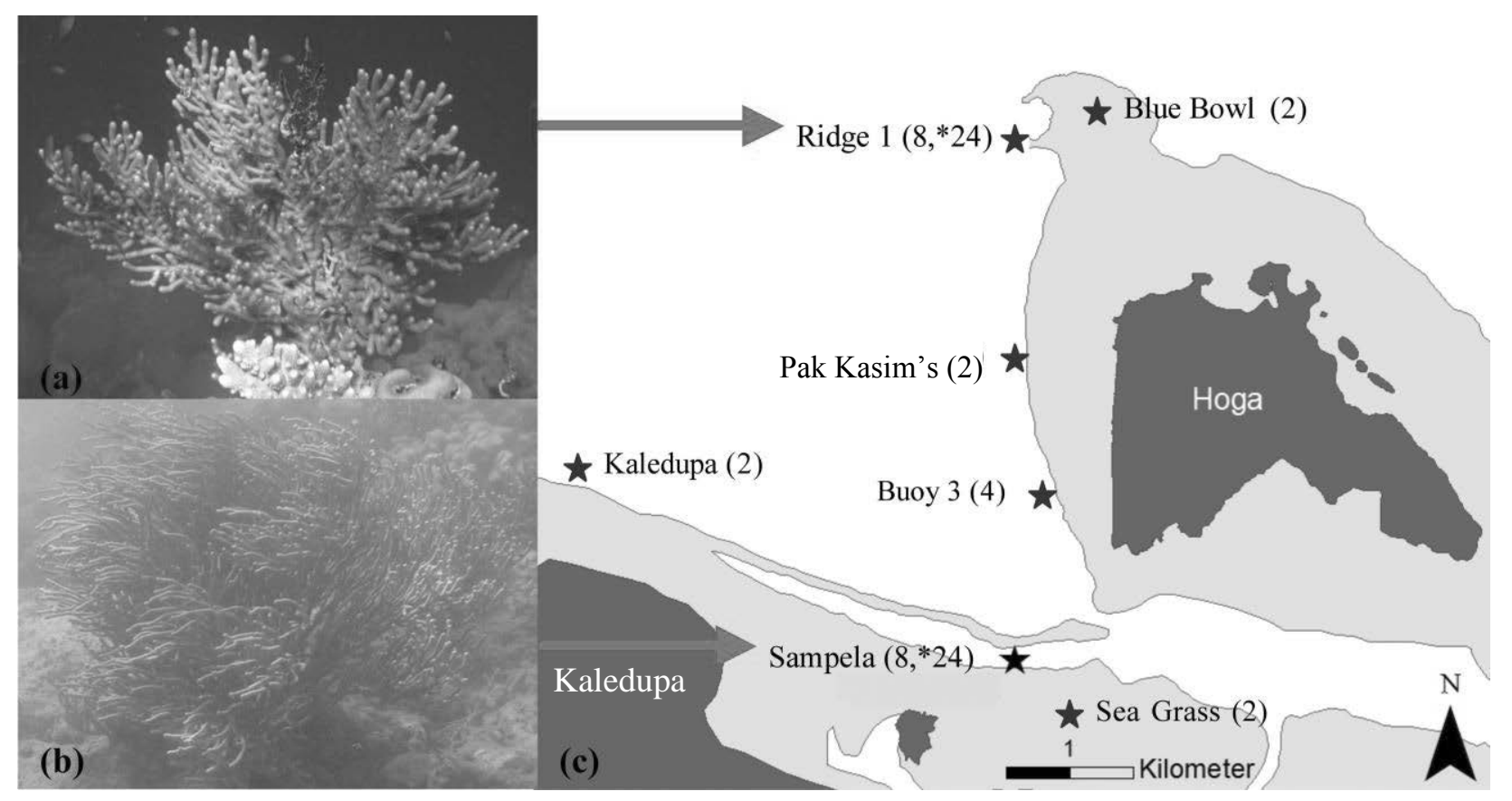

Figure 3.2. Isis hippuris morphotypes: (a) short branched predominantly planar or multiplanar colonies at the healthy site Ridge 1, and (b) long branched bushy colonies at the impacted site Sampela, with additional (c) collection localities within the WMNP. Sample number in brackets for molecular and asterisk for morphological analyses.

disturbance exist across reefs within the Wakatobi, providing a novel natural laboratory for studies of environmentally induced change on reef components.

Research was conducted between two sites spanning $5 \mathrm{~km}$ of anthropogenic and natural environmental gradients during July and August 2010. Ridge 1 (healthy) is an exposed reef ridge with high nutrient upwellings and water currents; Sampela (impacted) is a semi-lagoonal reef with low water flow and high turbidity. Sampela is situated ca. $400 \mathrm{~m}$ from a Bajo (sea gypsy) village of ca. 1600 people and subject to continuous marine resource exploitation and community waste disposal. Previously informative environmental variables driving morphotype distribution for the two study sites (summarized in Table 3.1 adapted from Table 2.1) were selected for further analyses. Light $\left(\mathrm{K}_{\mathrm{d}(\mathrm{PAR})}\right)$ was measured using $\mathrm{HOBO}^{\circledR}$ data loggers, turbidity (NTU) expressed as an inverse values, and chlorophyll- $a\left(\mu \mathrm{g} \mathrm{L}^{-1}\right)$ were measured using an $\mathrm{RBR}^{\circledR}$ XR-420 CTD data logger, a General Oceanics ${ }^{\circledR}$ flow meter was used to measure water flow velocity, and sediment grain size was estimated using Retsch Technology ${ }^{\circledR}$ test sieves, with logarithmically converted diameters expressed as phi $(\Phi)$ and classified using the Wentworth scale (Wentworth 1922). Environmental variables, with the exception of latitude and longitude, 
Table 3.1. Environmental characteristics of the two study sites in the WMNP, Indonesia. All values expressed as mean $( \pm \mathrm{SE})$. Data summarised of significant variables from Table 2.1.

\begin{tabular}{lll}
\hline Parameter Recorded & \multicolumn{2}{l}{ Mean value \pm SE (where appropriate) } \\
\cline { 2 - 3 } Site & Sampela & Ridge 1 \\
\hline Latitude $(\mathrm{S})$ & $005^{\circ} 29^{\prime} 01^{\prime \prime}$ & $005^{\circ} 26^{\prime} 57^{\prime \prime}$ \\
Longitude $(\mathrm{E})$ & $123^{\circ} 45^{\prime} 08^{\prime \prime}$ & $123^{\circ} 45^{\prime} 38^{\prime \prime}$ \\
Temperature $\left({ }^{\circ} \mathrm{C}\right.$ min-max) & $25.61-29.36$ & $24.06-28.07$ \\
Light $\left(\mathrm{K}_{\mathrm{d}(\mathrm{PAR})} \min -\mathrm{max}\right)$ & $0.31-3.14$ & $0.1-1.56$ \\
Flow $(\mathrm{cm} / \mathrm{s})$ & $5.02 \pm 2.18$ & $30.54 \pm 2.61$ \\
Chlorophyll- $a\left(\mu \mathrm{g} 1^{-1}\right)$ & $0.3 \pm 0.01$ & $0.35 \pm 0.03$ \\
Turbidity $(\mathrm{NTU})$ & $4.38 \pm 1.80$ & $0.17 \pm 0.33$ \\
Sedimentation $\left(\mathrm{g} \mathrm{d}^{-1}, \mathrm{n}=12\right)$ & $3.28 \pm 0.26$ & $1.16 \pm 0.07$ \\
Sediment grain size $(\Phi, \mathrm{n}=12)$ & $5[31.25-62.5 \mu \mathrm{m}]$ & $1[0.5-1 \mathrm{~mm}]$ \\
\hline
\end{tabular}

were edited visually with significant outliers removed, and entered into statistical models as raw values.

\subsubsection{Sample Collection}

Isis hippuris colonies were sampled from the healthy site Ridge $1(\mathrm{n}=24)$ and the anthropogenically impacted site Sampela $(n=24$; total $n=48)$, where the two distinct morphotypes at densities of 18 and 6 colonies per $10 \mathrm{~m}^{-2}$ respectively were previously documented (Chapter 2; Figure 3.3). A further twelve clippings were taken from five additional sites (Blue Bowl n =2; Pak Kasim's n =2; Buoy 3 n =4; Kaledupa $n=2$; Sea Grass beds $n=2$; Figure 3.2c) to investigate and compare genetic differences between colonies within the study area. Sample numbers for molecular analyses were low due to financial constraints, yet provide valuable insights for further study. All colonies were randomly selected within $2-5 \mathrm{~m}$ depth and a minimum of $10 \mathrm{~m}$ distance apart to avoid sampling asexual clone mates. Each colony was subject to in situ scaled digital photography using a Canon IXUS 900Ti, WP-DC7 u/w housing and INON UWL-105 AD x 0.51 lens, with duplicate samples preserved in $96 \%$ EtOH and Guanidinium solution for morphological and molecular analyses respectively. Colonies were photographed both parallel and overhead for planar and bushy colonies as appropriate with a ruler for scale. 


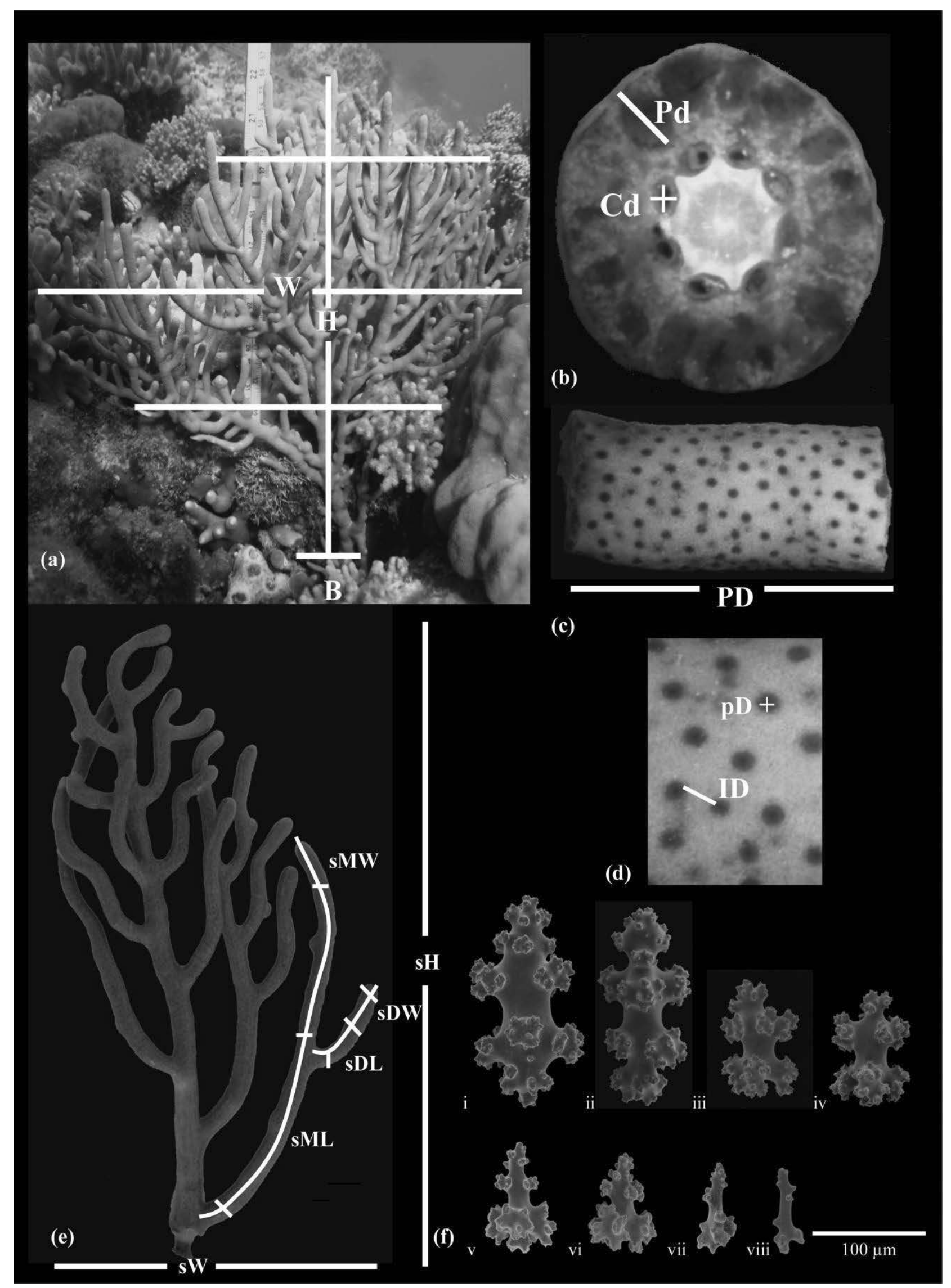


Figure 3.3 (Previous page). Isis hippuris morphological trait measurements of the (a) colony; (b, c, d) canal and polyp dynamics; (e) sub-colony (branching) dynamics; (f) sclerites site/morphotype comparisons of $\mathrm{i}$ and ii spindles, iii and iv capstan 7-radiates, $\mathrm{v}$ - viii clubs from Ridge 1 and Sampela respectively. All abbreviations are described in Table 3.2.

\subsubsection{Data Analyses}

\section{Morphological Measurements}

Population comparisons of morphological traits were conducted on 24 I. hippuris colonies from both Ridge 1 and Sampela (total 48 colonies). A total of 57 morphological traits were quantified and divided into 32 macro- and 25 micro-morphological traits (Figure 3.3; Table 3.2). Due to the variable nature of I. hippuris colonies (planar, multiplanar or bushy), particularly between sites, whole colony height $[\mathrm{H}]$, mean width $[\mathrm{W}]$ taken equidistant apart, and colony spread [CS] were measured with CS as the mean of two measurements taken above the colony. Branch tips [T], mid main branch $[\mathrm{M}]$ and base $[\mathrm{B}]$ width were also recorded but limited access to the latter meant data were omitted from further analyses $(n=13 \& 5$ for Ridge $1 \&$ Sampela respectively). Colony subsections of $\sim 20 \mathrm{~cm}$ in height were selected for further comparable macro-morphometric analyses: sub height $[\mathrm{sH}]$, mean width $[\mathrm{sW}]$, and projected sub-colony area [PA] estimated by $\mathrm{sH} \times \mathrm{sW}$ which was then used to calculate sub-colony porosity $[\mathrm{Po}]$ as a ratio of $\mathrm{PA}$ and the projected branch area $[\mathrm{PBA}]$ itself the total branch length multiplied by the mean branch thickness (see below). Branch articulation was assessed using a hierarchical generation ordering system (Lasker et al. 2003, Sánchez \& Lasker 2003, Sánchez et al. 2003a) where each branch was ascribed as either a 'mother' branch or 'daughter' branch, the latter emerging from the former. As the colony develops, daughter branches may also become mother branches (e.g. second generation mother branch; see Figure 3.3) quantified as follows: mother branch length [sML], mean mother branch width [sMW], daughter branch length [sDL], mean daughter branch width [sDW], total branch number [sTB\#], total branch length [sTBL], and mean branch width [MBW]. Branch surface area was calculated on the geometric approximation of a cylinder from branch length and mean width as the radius, with subsequent polyp density [PD] per $\mathrm{cm}^{2}$. Twenty random measurements were made of both polyp diameter ([pD] mean of 2 measurements see Figure 3.3) and inter-polyp distance [ID]. All polyp, branch cross-section and canal [C\#/Cd; Cd see Figure 3.3] quantification were visualised under an Olympus $\mathrm{SZX} 16^{\circledR}$ stereomicroscope at 10x magnification with $0.5 \mathrm{x}$ objective.

Isis hippuris has considerable diversity in sclerite form (Simpson 1906, Bayer \& Stefani 1987, Fabricius \& Alderslade 2001). For consistency, only those represented in all test colonies were 


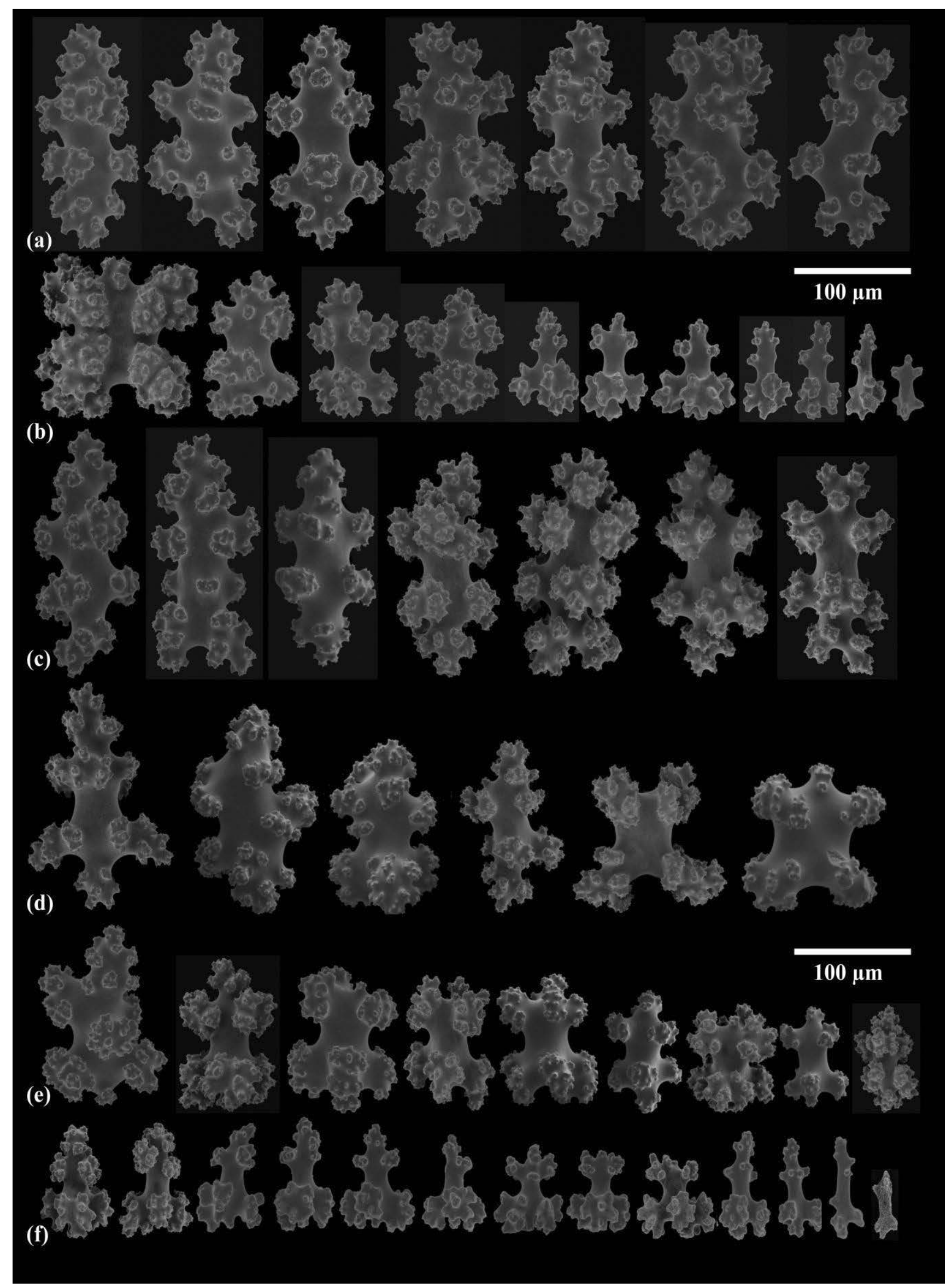


Figure 3.4 (Previous page) Scanning electron micrographs showing sclerite diversity of Isis hippuris from (a - b) Ridge 1 and (c - f) Sampela within the WMNP. Inner coenenchyme spindles (a, c, d), surface capstans and clubs (b, e, f). Small rods at the end of both (a \& f).

selected for quantitative analyses. Length and mean width of three measurements were made on 20 randomly selected sclerites per sclerite type; surface clubs [CL1/2, CW1/2]; and sub-surface capstans [7-radiates: $\mathrm{CaL} / \mathrm{W}]$ and spindles [SL/W](Figure 3.3). Additional sclerite diversity is shown in Figure 3.4. Sclerites were removed by dissolving the surrounding tissue in $5 \%$ sodium hypochlorite solution and visualized using optical microscopy (Olympus BX51 ${ }^{\circledR}$ ) and scanning electron microscopy (SEM) which was performed on a Hitachi S-800 SEM at the University of Hawai'i at Mānoa, USA. All micro-morphological measurements and sclerite preparation were taken $2 \mathrm{~cm}$ below the branch tip to avoid underdeveloped traits due to sub-apical branch growth (Lasker et al. 2003) and photographed using an Olympus 3.3MPX ${ }^{\mathrm{TM}}$ camera and Rincon software (ImagingPlanet ${ }^{\circledR}$ ). All macro- and micro-morphological characteristics were measured using ImageJ64 (Abràmoff et al. 2004).

Phenotypic traits were analysed using routines within the PRIMER-E v6.1.12 statistical package (Clarke and Gorley 2006), with PERMANOVA+ v1.02 extension (Anderson 2001). Specifically, character traits (untransformed) were simultaneously correlated in a Draftsman plot to eliminate uninformative traits $(P>0.95)$ and to establish appropriate transformation in downstream analyses (Clarke and Ainsworth 1993). Informative phenotypic trait data were subsequently standardized and a 'zero-adjusted' Bray-Curtis similarity matrix (Clarke et al. 2006b) constructed for tests of morphological divergence between the two sites; Ridge 1 and Sampela. A single-factor model with 9999 permutations (PERMANOVA; Anderson 2001) was performed and further visualised utilizing non-metric multidimensional scaling (nMDS) and constrained canonical analysis of principal coordinates (CAP; Anderson \& Willis 2003). Informative traits contributing most to the dissimilarities between sites, thus specific morphotypes were investigated using similarity percentages (SIMPER; Clarke 1993) and displayed as a vector overlay on the CAP ordination.

The relationship between I. hippuris morphotypes and their environment was investigated using nonparametric multivariate regression (McArdle \& Anderson 2001) with the DISTLMforward routine (Anderson 2003). Based on a Euclidean distance matrix, all raw environmental variable data were normalised (Table 3.1) and significance tested using 9999 permutations (Anderson 2001). 
Table 3.2. Isis hippuris morphological traits summary table. All values expressed as metric or counts $( \pm$ SE). Asterisk $(*)$ indicate significantly $(<P 0.05)$ informative traits selected for multivariate analyses. $\dagger$ Depicts low sample size.

\begin{tabular}{|c|c|c|c|c|c|c|c|c|c|}
\hline \multirow{2}{*}{\multicolumn{2}{|c|}{ Morphological Trait }} & \multirow{2}{*}{$\begin{array}{l}\text { Measures/ } \\
\text { Counts }\end{array}$} & \multicolumn{2}{|c|}{ Dimensions $( \pm \mathrm{SE})$} & \multirow{2}{*}{\multicolumn{2}{|c|}{ Morphological Trait }} & \multirow{2}{*}{$\begin{array}{c}\text { Measures/ } \\
\text { Counts }\end{array}$} & \multicolumn{2}{|c|}{ Dimensions ( \pm SE) } \\
\hline & & & Ridge 1 & Sampela & & & & Ridge 1 & Sampela \\
\hline \multirow{2}{*}{\multicolumn{5}{|c|}{$\begin{array}{l}\text { Macromorphology (cm) } \\
\text { Colony }\end{array}$}} & \multirow{2}{*}{\multicolumn{5}{|c|}{$\begin{array}{l}\text { Micromorphology }(\mathrm{mm}) \\
\text { sub Colony }\end{array}$}} \\
\hline & & & & & & & & & \\
\hline${ }^{*} \mathrm{H}$ & Colony Height & 48 & $49.74 \pm 3.99$ & $58.78 \pm 3.10$ & $* \mathrm{PD}$ & Polyp Density & 31,761 & $88.23 \pm 2.6$ & $100.21 \pm 5.32$ \\
\hline$* \mathrm{~W}$ & Colony Mean Width & 48 & $39.65 \pm 2.24$ & $58.03 \pm 2.94$ & $\mathrm{Pd}$ & Polyp Depth & 398 & $0.08 \pm 0.001$ & $0.08 \pm 0.002$ \\
\hline$*_{w 1}$ & width 1 & 144 & $40.53 \pm 3.24$ & $65.31 \pm 5.35$ & $\mathrm{pD}$ & Polyp Diameter & 1920 & $0.04 \pm 0.002$ & $0.03 \pm 0.001$ \\
\hline$*_{w 2}$ & width 2 & 144 & $57.02 \pm 3.06$ & $76.74 \pm 3.6$ & ID & Inter-polyp Distance & 1920 & $0.05 \pm 0.002$ & $0.05 \pm 0.001$ \\
\hline$*_{w 3}$ & width 3 & 144 & $22.0 \pm 1.35$ & $31.86 \pm 1.93$ & $\mathrm{Cd}$ & Canal diameter & 1,920 & $0.02 \pm 0.001$ & $0.02 \pm 0.002$ \\
\hline$*_{\mathrm{CS}}$ & Colony overhead Spread & 48 & $45.5 \pm 5.10$ & $78.86 \pm 3.10$ & $\mathrm{C \#}$ & Canal\# & 1,677 & $8.17 \pm 0.14$ & $8.33 \pm 0.21$ \\
\hline$* c s 1$ & colony spread 1 & 48 & $52.06 \pm 5.34$ & $78.86 \pm 3.92$ & & & & & \\
\hline${ }_{*}^{*} \operatorname{cs} 2$ & colony spread 2 & 48 & $38.94 \pm 4.85$ & $62.12 \pm 3.99$ & Sclerites & & & & \\
\hline B & Colony Base Width & 18 & $3.47 \pm 0.40$ & $5.43 \pm 0.64$ & $* \mathrm{CL} 1$ & Club Length 1 & 960 & $0.073 \pm 0.001$ & $0.068 \pm 0.001$ \\
\hline$* \mathrm{M}$ & Colony Mid Branch Width & 48 & $1.10 \pm 0.08$ & $1.53 \pm 0.48$ & $* \mathrm{CW} 1$ & Club Mean Width 1 & 48 & $0.021 \pm 0.000$ & $0.020 \pm 0.000$ \\
\hline \multirow[t]{2}{*}{$* \mathrm{~T}$} & Colony Tip Branch Width & 960 & $0.63 \pm 0.02$ & $0.95 \pm 0.05$ & $c 1 w 1$ & clwidth 1 & 960 & $0.022 \pm 0.001$ & $0.021 \pm 0.001$ \\
\hline & & & & & $\operatorname{clw2} 2$ & clwidth 2 & 960 & $0.012 \pm 0.000$ & $0.011 \pm 0.001$ \\
\hline \multicolumn{2}{|c|}{ sub Colony } & & & & $c 1 w 3$ & clwidth 3 & 960 & $0.030 \pm 0.002$ & $0.028 \pm 0.001$ \\
\hline$*_{\mathrm{sH}}$ & sHeight & 48 & $10.8 \pm 0.33$ & $11.55 \pm 0.24$ & $* \mathrm{CL} 2$ & Club Length 2 & 960 & $0.072 \pm 0.000$ & $0.068 \pm 0.002$ \\
\hline${ }_{\mathrm{s}}^{\mathrm{sW}}$ & sMean Width & 48 & $3.15 \pm 0.21$ & $2.66 \pm 0.17$ & $* \mathrm{CW} 2$ & Club Mean Width 2 & 48 & $0.033 \pm 0.000$ & $0.031 \pm 0.001$ \\
\hline$s w 1$ & swidth 1 & 144 & $2.50 \pm 0.14$ & $2.46 \pm 0.15$ & $c 2 w 1$ & c2width 1 & 960 & $0.032 \pm 0.001$ & $0.030 \pm 0.001$ \\
\hline$s w 2$ & swidth 2 & 144 & $4.18 \pm 0.28$ & $3.37 \pm 0.22$ & $c 2 w 2$ & $c 2$ width 2 & 960 & $0.016 \pm 0.000$ & $0.016 \pm 0.001$ \\
\hline sw3 & swidth 3 & 144 & $2.76 \pm 0.32$ & $1.94 \pm 0.23$ & $c 2 w 3$ & c2width 3 & 960 & $0.051 \pm 0.001$ & $0.047 \pm 0.002$ \\
\hline sML & sMean Mother Length & 48 & $8.41 \pm 0.33$ & $9.77 \pm 0.35$ & $* \mathrm{CaL}$ & Capstan Length & 960 & $0.114 \pm 0.004$ & $0.101 \pm 0.002$ \\
\hline *sMW & sMean Mother Width & 48 & $0.44 \pm 0.02$ & $0.55 \pm 0.02$ & $* \mathrm{CaW}$ & Capstan Mean Width & 48 & $0.075 \pm 0.001$ & $0.066 \pm 0.001$ \\
\hline$m w 1$ & mwidth 1 & 144 & $0.51 \pm 0.01$ & $0.62 \pm 0.03$ & *cawl & cawidth 1 & 960 & $0.086 \pm 0.004$ & $0.076 \pm 0.003$ \\
\hline$m w 2$ & mwidth 2 & 144 & $0.48 \pm 0.01$ & $0.57 \pm 0.02$ & *caw 2 & cawidth 2 & 960 & $0.041 \pm 0.003$ & $0.036 \pm 0.020$ \\
\hline$m w 3$ & mwidth 3 & 144 & $0.42 \pm 0.01$ & $0.54 \pm 0.02$ & *aw3 & cawidth 3 & 960 & $0.090 \pm 0.003$ & $0.080 \pm 0.002$ \\
\hline$*_{\mathrm{sDL}}$ & sMean Daughter Branch Length & 48 & $3.08 \pm 0.21$ & $4.44 \pm 0.18$ & SL & Spindle Length & 960 & $0.164 \pm 0.003$ & $0.162 \pm 0.003$ \\
\hline$*_{\mathrm{sDW}}$ & sMean Daughter Branch Width & 48 & $0.42 \pm 0.01$ & $0.48 \pm 0.01$ & *SW & Spindle Mean Width & 48 & $0.069 \pm 0.001$ & $0.065 \pm 0.001$ \\
\hline$d w 1$ & dwidth 1 & 480 & $0.44 \pm 0.01$ & $0.51 \pm 0.01$ & $s w 1$ & swidth 1 & 960 & $0.038 \pm 0.002$ & $0.037 \pm 0.002$ \\
\hline$d w 2$ & $d$ width 2 & 480 & $0.41 \pm 0.01$ & $0.49 \pm 0.01$ & $s w 2$ & swidth 2 & 960 & $0.080 \pm 0.003$ & $0.076 \pm 0.002$ \\
\hline$d w 3$ & dwidth 3 & 480 & $0.38 \pm 0.01$ & $0.45 \pm 0.01$ & $*_{s w 3}$ & swidth 3 & 960 & $0.081 \pm 0.003$ & $0.077 \pm 0.002$ \\
\hline *MBW & Mean Branch Width & 48 & $0.43 \pm 0.01$ & $0.52 \pm 0.01$ & & & & & \\
\hline *sTB\# & sTotal Branch\# & 48 & $13.5 \pm 1.10$ & $10.83 \pm 1.42$ & Total & & 59,328 & & \\
\hline sTBL & sTotal Branch Length & 48 & $51.86 \pm 3.49$ & $52.57 \pm 3.76$ & & & & & \\
\hline *PA & Projected sub-colony Area & 48 & $34.28 \pm 2.65$ & $30.79 \pm 2.03$ & & & & & \\
\hline *PBA & Projected Branch Area & 48 & $22.41 \pm 1.69$ & $26.96 \pm 2.06$ & & & & & \\
\hline *Po & Porosity & 48 & $1.61 \pm 0.10$ & $1.19 \pm 0.06$ & & & & & \\
\hline
\end{tabular}




\section{Genetics Analyses}

Genomic DNA of I. hippuris and endosymbiotic dinoflagellates (for the latter see Chapter 4) were extracted from 28 colonies, 8 from each of the two test sites $(n=16)$ and 12 from additional site populations for area and morphotype comparison as described above. Approximately $2-3 \mathrm{~mm}$ of fresh soft tissue was immediately cut and stored in $400 \mu \mathrm{l}$ Guanidinium lysis buffer (4 M guanidinium isothiocyanate, 0.05 M Tris pH 7.6, 0.01 M EDTA, 0.07 M Sarkosyl, $\beta$-mercaptoethanol $1 \% \mathrm{v} / \mathrm{v}$ ) (Pochon et al. 2001) for 14 days at room temperature during transit from the field, with subsequent storage at $4^{\circ} \mathrm{C}$. Preserved samples were incubated at $72^{\circ} \mathrm{C}$ for $20 \mathrm{~min}$, vortexed prior, during and after incubation, then centrifuged at $16,000 \mathrm{~g}$ for $5 \mathrm{~min}$. The resulting DNA-containing supernatant was precipitated with an equal volume of $100 \%$ isopropanol, vortexed and stored over night at $-20^{\circ} \mathrm{C}$. DNA was pelleted via centrifugation at $16,000 \mathrm{~g}$ for $15 \mathrm{~min}$, washed with $70 \% \mathrm{EtOH}$, centrifuged for $10 \mathrm{~min}$, dried and resuspended in 0.1 M Tris Buffer $\mathrm{pH}$ 8. The DNA solution was placed on ice for 1 hour with frequent vortexing and stored at $-20^{\circ} \mathrm{C}$. DNA was visualized on $1 \%$ agarose gel. PCR amplifications of the ITS2 rDNA marker were conducted using the primers itsD (forward; 5'GTGAATTGCAGAACTCCGTG-3') and ITS2Rev2 (reverse; 5'CCTCCGCTTACTTATATGCTT-3') (Pochon et al. 2005, 2007, 2010). Total PCR volume was $50 \mu$ l constituting: $5.0 \mu \mathrm{L}$ of 10x PCR Buffer (Bioline), $2.0 \mu \mathrm{L}$ of $\mathrm{MgCl}_{2}(2 \mathrm{mM}), 1.0 \mu \mathrm{L}$ of each primer $(10 \mathrm{mM}), 1 \mu \mathrm{L}(2.5 \mathrm{mM}$ of each dATP, dCTP, dGTP, and dTTP), $0.2 \mu \mathrm{L}$ of Hotstart Immolase Taq polymerase (Bioline Incl., London, UK), $1.0 \mu \mathrm{L}$ of DNA, and $39 \mu \mathrm{L}$ of sterile water. Touchdown amplification was conducted as follows: denaturation at $95^{\circ} \mathrm{C}$ for $10 \mathrm{~min}, 25$ cycles at $94^{\circ} \mathrm{C}$ then $35 \mathrm{~s}$ at $65^{\circ} \mathrm{C}$ (reduction in annealing temperature of $0.5^{\circ} \mathrm{C}$ per cycle), and 2 min at $72^{\circ} \mathrm{C}$. A further 14 cycles of $30 \mathrm{~s}$ at $94^{\circ} \mathrm{C}, 35 \mathrm{~s}$ at $52^{\circ} \mathrm{C}, 2 \mathrm{~min}$ at $72^{\circ} \mathrm{C}$, and a final $10 \mathrm{~min}$ extension at $72^{\circ} \mathrm{C}$. All amplicons were purified using the QIAquick ${ }^{\mathrm{TM}}$ PCR Purification Kit (Qiagen), and separated by cloning for haplotype verification. Purified products were ligated into the pGEM $^{\circledR}-\mathrm{T}$ Easy vector ${ }^{\mathrm{TM}}$ (Promega), transformed into $\alpha$-Select Gold Efficiency ${ }^{\mathrm{TM}}$ competent cells (Bioline), with subsequent positive inserts verified by PCR using plasmid specific primers (M13). Positive inserts (8-12 per library) were purified with an ExoSAP-IT kit, sequenced in both directions using the ABI Prism Big Dye ${ }^{\mathrm{TM}}$ Terminator Cycle Sequencing Ready Reaction Kit and run on an ABI 3100 Genetic Analyzer (Perkin-Elmer Applied Biosystems, Foster City, CA, USA) at the University of Hawai'i at Mānoa, USA.

ITS2 clone libraries from 28 individuals were aligned using ClustalW2 (Thompson et al. 2002) and manually edited in Geneious Pro v.5.6.2 (Biomatters Ltd., NZ). A selection criterion of 
Table 3.3. ITS2 Accessions of octocoral outgroups used in the analyses.

\section{Taxon}

[Group: Alcyoniinans]

Family: Alcyoniidae Lamouroux 1812

Alcyonium digitatum Linnaeus 1758

[Group: Scleraxonians]

Family: Coralliidae Lamouroux 1812

Corallium rubrum Linnaeus 1758

Corallium sp. 1

Family: Paragorgiidae Kükenthal 1916

Paragorgia kaupeka Sánchez 2005

Sibogagorgia cauliflora Herrera, Baco \& Sánchez 2010

[Suborder: Holaxonians]

Family: Gorgoniidae Lamouroux 1812

Africagorgia schoutedeni Stiasny 1939

Gorgonia flabellum Linnaeus 1758

Leptogorgia violacea Pallas 1766

Lophogorgia [Synonym of Leptogorgia] euryale Bayer 1952

Pacifigorgia stenobrochis Valenciennes 1846

Pinnigorgia platysoma Nutting 1910

Pseudopterogorgia [Synonym of Antillogorgia] bipinnata Verrill 1864

Family: Plexauridae Gray 1859

Eunicea tourneforti Milne Edwards \& Haime 1857

Muriceopsis bayeri Sánchez 2001

[Suborder: Calcaxonians]

Family: Isididae Lamouroux 1812

Acanella weberi Nutting 1910

Acanella sp.

Isidella tentaculum Etnoyer 2008

Keratoisis zelandica Grant 1976

Lepidisis olapa Muzik 1978

Family: Primnoidae Milne Edwards 1857

Calyptrophora japonica Gray 1866

\section{GenBank Reference}

AF262347 McFadden et al. 2001

AF413059 Constantini et al. 2003

GQ358526 Herrera et al. 2010

GQ293292 Herrera et al. 2010

GQ293288 Herrera et al. 2010

AY587533 Aguilar \& Sánchez 2007a

AY587521 Aguilar \& Sánchez 2007a

AY587527 Aguilar \& Sánchez 2007a

AY587530 Aguilar \& Sánchez 2007a

AY587531 Aguilar \& Sánchez 2007a

AY587536 Aguilar \& Sánchez 2007a

AY587524 Aguilar \& Sánchez 2007a

EF490982 Grajales et al. 2007

AY587538 Aguilar \& Sánchez 2007a

FJ790943 Dueñas \& Sánchez 2009

FJ790921 Dueñas \& Sánchez 2009

FJ790944 Dueñas \& Sánchez 2009

FJ790939 Dueñas \& Sánchez 2009

FJ790908 Dueñas \& Sánchez 2009

EF090735 Aguilar \& Sánchez 2007a

identical sequences from two or more clone libraries was established to minimize the effect of intragenomic variation and/or PCR artefacts on further analyses. On average 4 - 6 host clones were recovered per library due to simultaneous recovery of both host and endosymbionts (see Chapter 4).

Estimates of genetic differentiation relative to morphotype were investigated via an analysis of molecular variance (AMOVA) with pairwise population comparisons $\left(\Phi_{\mathrm{ST}}\right)$ between sites using ARLEQUIN v.3.5 (Excoffier \& Lischer 2010). Haplotype $\left(h_{d}\right)$, nucleotide diversity $(\pi)$ and substitution rate (JC) were calculated with DNAsp v.5.0 (Librado \& Rozas 2009). A parsimony haplotype network with a 95\% confidence level and gaps treated as a fifth state was constructed using Network v.4.6.1.1 on sample sequences only. 


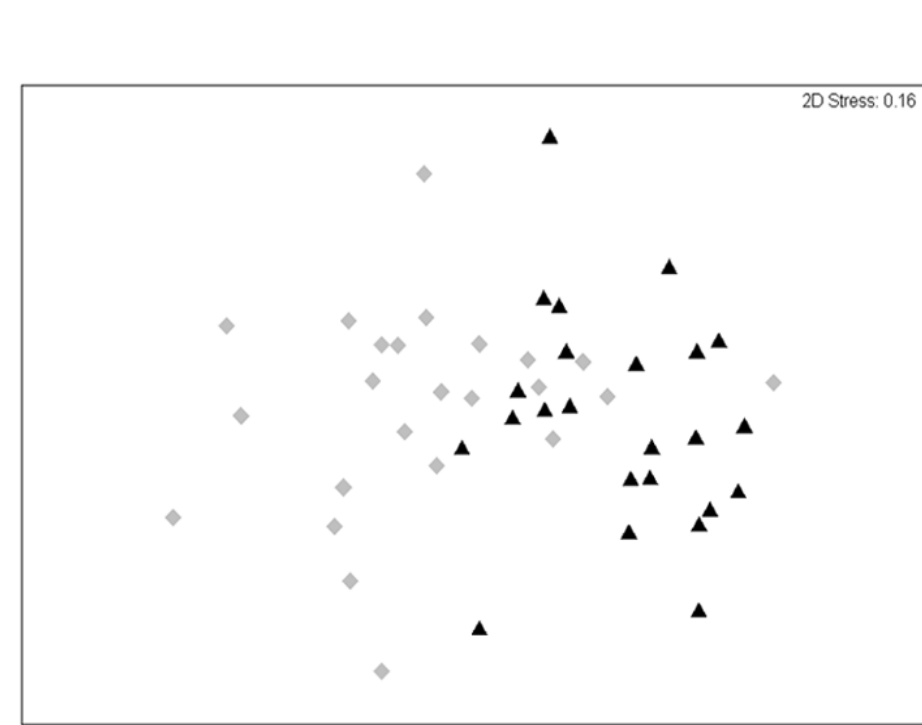

(a)

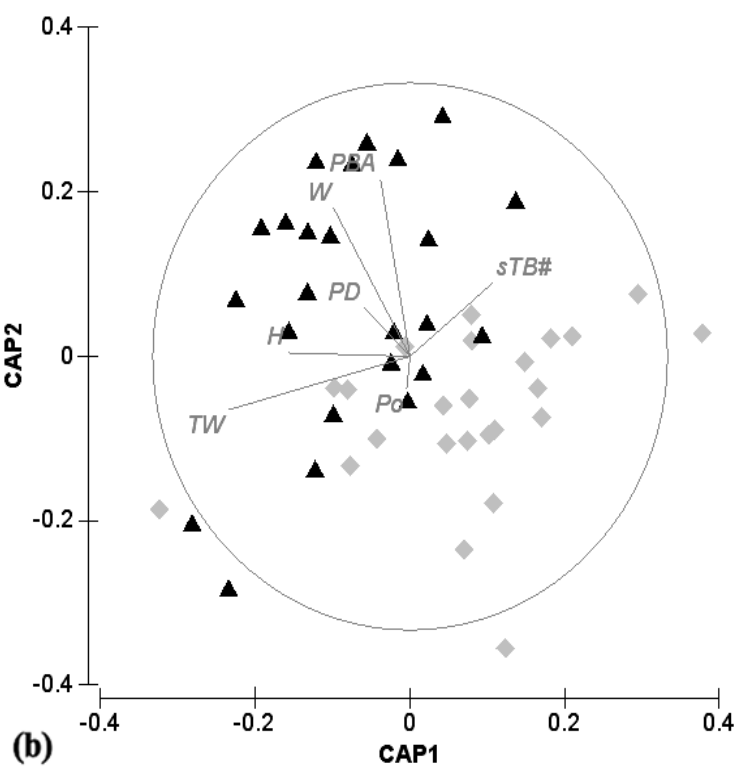

(b)

Figure 3.5. (a) Unconstrained and (b) constrained ordinations of Isis hippuris character traits between Ridge $1[\bullet]$ and Sampela $[\boldsymbol{\Delta}]$.

\section{ITS2 Predicted RNA Secondary Structure}

ITS2 RNA secondary structures were predicted to further investigate haplotype differences specifically between Ridge 1 and Sampela at a more conserved level. Alcyonium digitatum Linnaeus 1758 (Genbank Acc. \# AF262347; McFadden et al. 2001) was used as a template for conserved motif identification with subsequent constraints implemented into MFOLD (Zuker 2003) using default parameters. RNA was folded at $37^{\circ} \mathrm{C}$ and structures with the highest negative free energy values, thus stability, were selected, manually edited in 4SALE (Seibel et al. 2006, 2008) and visually annotated in VARNA (Darty et al. 2009).

Phylogenetic reconstructions between Isis haplotypes and twenty octocoral ITS2 outgroups obtained from GenBank (see Table 3.3) were conducted using the PHYML 2.1.0 (Guindon \& Gascuel 2003) and MrBayes 2.0.5 (Huelsenbeck \& Ronquist 2001) plugins within Geneious. Indels (insertion and deletion mutations) were considered phylogenetically informative and treated as separate characters using the 'simple indel coding' gap method (Simmons \& Ochoterena 2000) in GapCoder v.1.0 (Young \& Healy 2003). Maximum likelihood (ML) phylogeny was conducted using the best-fit model (JC) of nucleotide substitution as selected in jModelTest 2 (Darriba et al. 2012) through Akaike Information Criterion (AIC). Bayesian inference (BI) phylogeny was made with a JC69 substitution model and burn-in of 100,000. Phylogenetic trees were rooted with Paragorgia kaupeka Sánchez 2005 and node support values set at $\geq 70 \%$ for both ML and BI. 
Table 3.4. Isis hippuris ITS 2 haplotype sequence view from the seven test sites within the WMNP. Each sequence represents haplotypes present in each sample per site. Colour codes depict gaps (lilac), transitions (red), and transversions (yellow).

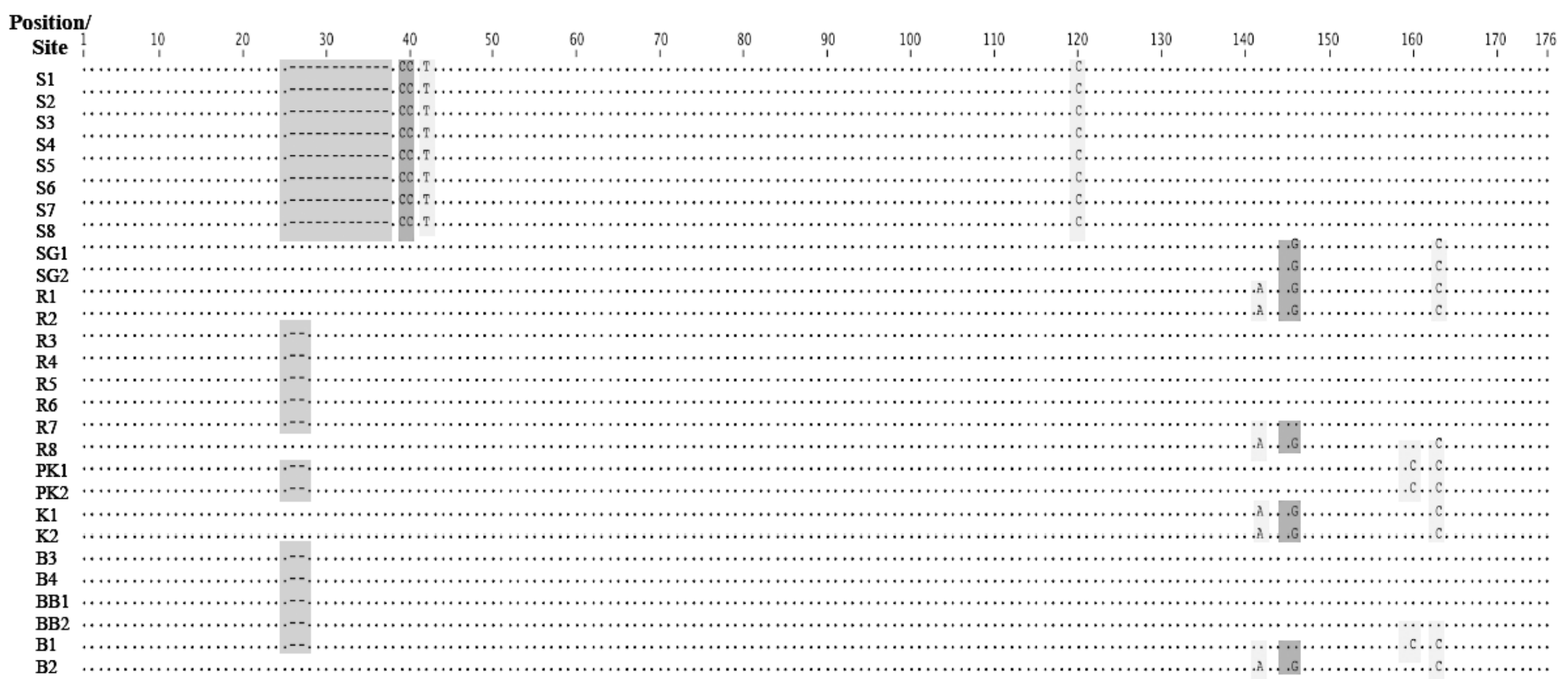


Table 3.5. AMOVA of genetic structure between sites within the WMNP from both cloned and sample sequences. R1 denotes Ridge 1; $\mathrm{S}$ denotes Sampela. $* P<0.001$ significant.

\begin{tabular}{|c|c|c|c|c|c|}
\hline Source of Variation & $d f$ & SS & $\begin{array}{l}\text { Variance } \\
\text { Component }\end{array}$ & Variance $\%$ & $\Phi_{S T}$ \\
\hline \multicolumn{6}{|l|}{7 Populations: Clones } \\
\hline Among populations & 6 & 122.248 & $V_{a}=1.237$ & 80.97 & $0.80974 *$ \\
\hline Within populations & 113 & 32.844 & $V_{b}=0.291$ & 19.03 & \\
\hline Total & 119 & 155.092 & 1.528 & & \\
\hline \multicolumn{6}{|l|}{7 Populations: Samples } \\
\hline Among populations & 6 & 33.768 & $V_{a}=1.401$ & 76.83 & $0.76831^{*}$ \\
\hline Within populations & 21 & 8.875 & $V_{b}=0.423$ & 23.17 & \\
\hline Total & 27 & 42.643 & 1.824 & & \\
\hline \multicolumn{6}{|c|}{2 Populations (R1 \& S): Samples } \\
\hline Among populations & 1 & 17.688 & $V_{a}=2.161$ & 84.32 & $0.84321^{*}$ \\
\hline Within populations & 14 & 5.625 & $V_{b}=0.402$ & 15.68 & \\
\hline Total & 15 & 23.312 & 2.563 & & \\
\hline
\end{tabular}

\subsection{RESULTS}

\subsubsection{Morphometrics}

Of the 57 morphological traits measured, 32 were selected for further analyses (Table 3.2). Based on these traits, PERMANOVA revealed significant differences between morphotypes across the two study sites Sampela and Ridge 1 (pseudo- $F=14.489 ; P<0.0001$ ), further corroborated by the CAP analysis $\left(\delta^{2}=0.995, P=<0.0001\right.$; Figure $\left.3.5 \mathrm{~b}\right)$ with $89 \%$ variance $(\%$ var.) as the total variance explained by the first $m$ PCO axes. Prominent morphological traits contributing most to dissimilarities between sites were primarily at the colony level (TW, W, H, PBA, Po, \& sTB\#) with the exception of a higher polyp density (PD) at Sampela (Figure 3.5). From both Figure $3.5 \mathrm{~b}$ and Table 3.2 it is clear that larger colonies present at Sampela have a reduction in branch density yet larger colony size and spread (PBA, sTB\#, Po, and H, W, TW respectively). Branches were also consistently longer and thicker including the branch tips at Sampela, however polyp parameters were relatively invariable despite significantly high polyp density. It is noteworthy that all sclerite trait measurements were consistently smaller at Sampela (Table 3.2), particularly capstans (Figure 3.3f.iii, iv \& 3.4 for variability) were variable throughout I. hippuris distribution (Simpson 1906, Bayer \& Stefani 1987, Fabricius \& Alderslade 2001). Yet irrespective of pre-treatment the magnitude of differences between sclerite measurements were not that of the colony level. Nevertheless, separation and re-analyses under the same models for macro (e.g., colony and sub-colony: pseudo- $F=15.255 ; P<0.0001$; 


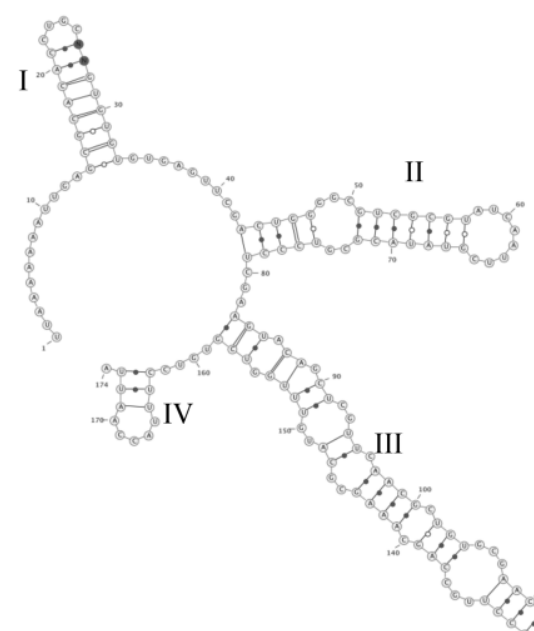

(B) $\Delta \mathrm{G}=-43.30 \mathrm{kcal} / \mathrm{mol}, 37^{\circ} \mathrm{C}$

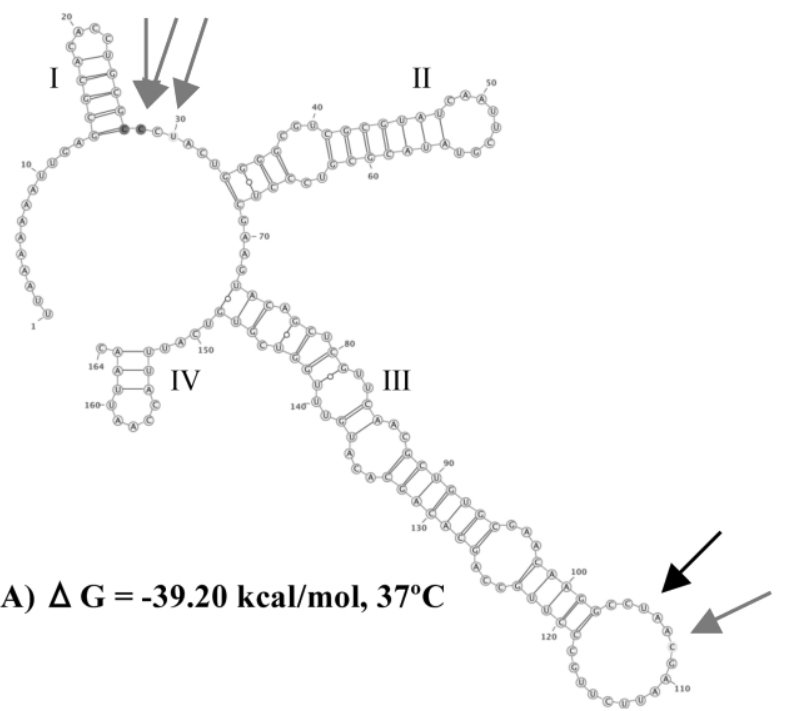

A

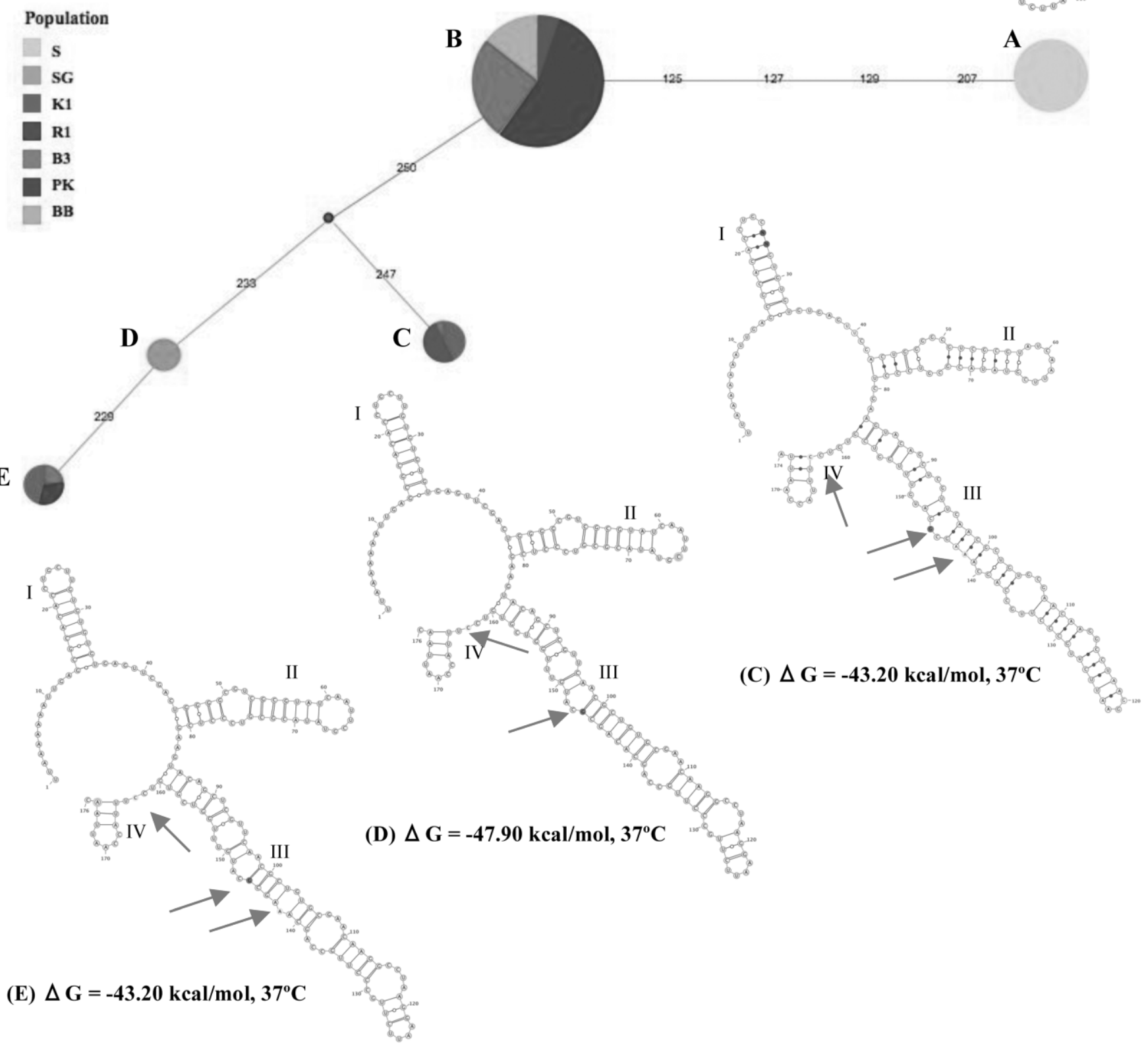


Figure 3.6. (Previous page) Isis haplotype network with corresponding ITS2 RNA predicted secondary structure relative to haplotype (A - E, see also Figure 3.7) and enthalpy values according to MFOLD. Roman numerals (I - IV) represent helices; red and black arrows indicate point mutations and loop differences respectively. Coloured bases according to transitions (red), transversions (yellow) and gaps (lilac), Table 3.5. Haplotype circle diameters are proportional to identical clone sequences.

CAP $\delta 2=0.976, P=<0.0001,91 \%$ var.) and micro (e.g. sclerite: pseudo- $F=11.727 ; P<0.001$; CAP $\delta^{2}=0.996, P=<0.0001,85 \%$ var.) measurements did not significantly alter results from the full model, demonstrating a lack of redundancy in selected character traits.

Results from the distance-based nonparametric regression (DISTLMforward) revealed that turbidity and sediment load explained $27.31 \%$ (pseudo- $F=5.100 ; P<0.001$ ) of morphotype differences between the two sites.

\subsubsection{ITS2 Sequence Diversity}

Of the 28 Isis samples 120 clones were recovered; Ridge (34), Sampela (29), Sea Grass (9), Kaledupa (12), Buoy 3 (18), Pak Kasim's (10), and Blue Bowl (8). ITS2 sequences revealed five haplotypes: 1-3 per sample population with up to 8 substitutions (Table 3.4, Figure 3.6). In keeping with morphological traits, colonies found at Sampela were significantly different from all other sample sites (Table 3.5, Figure 3.6). Haplotype diversity was greatest across Hoga Island with overall haplotype $\left(h_{d}\right)$ and nucleotide diversity $(\pi)$ measured as 0.780 and 0.0197 respectively with just (JC) 0.0313 substitutions per site. Population division was strongly inferred by all AMOVA models (Table 3.5) and haplotype network analysis, the latter showing no evidence of reticulation through homoplasy (Figure 3.6). Curiously, the single haplotype present in the sea-grass beds (D) shared no nucleotide differences with Sampela (A) despite its relatively close proximity, however little can be determined without greater sampling effort. Pairwise $\Phi_{\text {ST }}$ estimates of ITS2 sequences from Sampela ranged from 1.000 (Sampela vs. Sea Grass, Kaledupa, Pak Kasim's, Blue Bowl, Buoy 3) to 0.843 (Sampela vs. Ridge 1; $P<0.0001$ in all cases), and from 0.467 (Ridge 1 vs. Pak Kasim's; $P<0.05$ ) to no structure (Ridge 1 vs. Blue Bowl and Buoy 3) at Ridge 1. Note, such values, in particular the $P$ - values, should be treated with caution. Low sample sizes reduce fine-scale structure detection, thus more data would likely yield greater insight into the level of haplotype and nucleotide diversity observed across sites with an increase in taxonomic certainty. 
ITS2 predicted RNA secondary structure analyses revealed minimal variation between haplotypes with the exception of Sampela (Figure 3.6) providing greater confidence in phenotypic trait differences. Clones were collapsed into haplotypes per sample for phylogenetic analyses. Phylogenetic topologies using Maximum Likelihood and Bayesian Inference were very similar including all outgroups and unambiguously identical with regard to WMNP haplotypes (Figure 3.7). Branch support was typically stronger with BI particularly regarding outgroup species where recognised taxonomic suborders and groups were distinct. Phylogenetic uncertainty leading to the addition of multiple outgroups, confirmed Isis sequences from the WMNP were not grouped with morphologically described sister taxa within the Isididae (highlighted red, Figure 3.7). Reducing the outgroup number did not alter the integrity of the phylogenetic signal, in fact irrespective of model or selected root Alcyonium digitatum Linnaeus 1758 consistently positioned directly above Isis haplotypes.

\subsection{DISCUSSION}

Isis hippuris morphotypes were clearly defined both morphologically and genetically between the two sites $\left(\Phi_{\mathrm{ST}}=0.7683, P<0.001\right)$. Even with a reduced ITS2 sample size, corroboration of both morphometric and molecular results reveal a powerful indication that divergence has or is taking place, the nature of which is unclear. Multivariate trait integration at the colony level (including branching parameters), polyp density and sclerite size define significant differences between morphotypes indicative of trait dependency, yet polyp dimension and canal width appear canalised (genetically fixed). Nevertheless, inherent phenotypic plasticity and/or disruptive selection may enhance the success of two phenotypes particularly across contrasting environments. Trophic level interaction through differential light and nutrient exposure may drive such phenotypic differences, further reinforced by population structure by asexual fragmentation and external brooding (Chapter $2 \& 4$ ). Taxonomic assignment maybe tenuous, however, considering the partial adherence of morphotypes to previously described species within the Isis genus in addition to polyphyly within the Isididae.

\subsubsection{Isis: 1 species or 2?}

Of the 48 colonies (28 used for genetic analyses) studied here and 1094 recorded in Chapter 2, it cannot be said with confidence that I. hippuris morphotypes at either site within the WMNP adhere to the descriptions as outlined for I. reticulata (Nutting 1910, Kükenthal 1919, 1924, Stiasny 1940, Mai-Bao-Thu 1971) or I. minorbrachyblasta (Zou et al. 1991), and may just be an artifact of depth in those previously described. I. hippuris contrasts with I. reticulata on the basis 


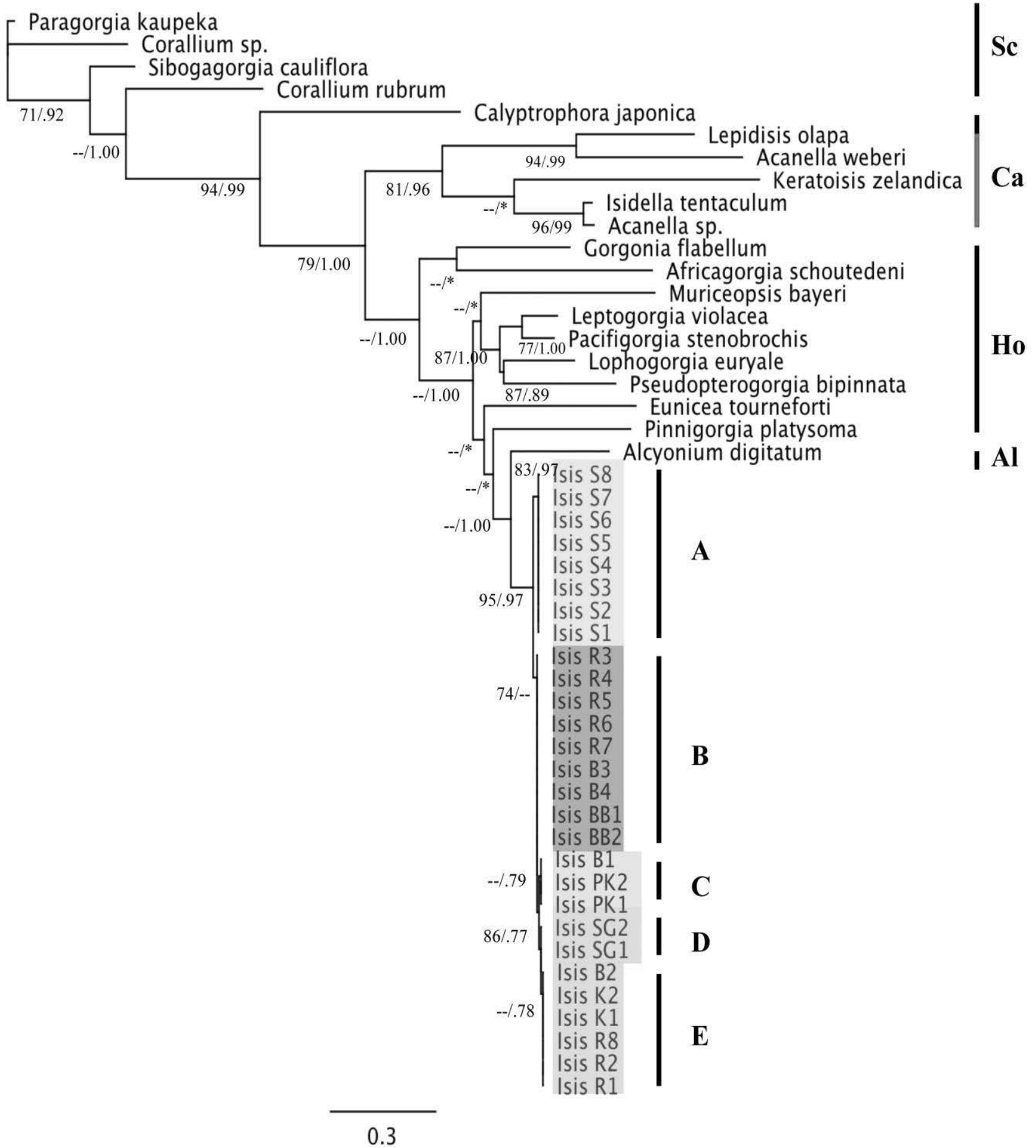

Figure 3.7. Phylogram based on maximum likelihood (ML) analyses of the ITS2 region from twenty Octocoral taxa in GenBank and Isis haplotypes within the WMNP. Branch numbers represent ML/BI support with low values expressed as a hyphen (--) $\leq 70 \%$ and asterisk (*) indicative of differences from MrBayes phylogenetic inference. Letters $\mathrm{Sc}=$ Scleraxonia, $\mathrm{Ca}=$ Calcaxonia, Ho $=$ Holaxonia, $\mathrm{Al}=$ Alcyoniina, and A - E represent Isis haplotypes as depicted in Figure 3.6. 
of short thick branches in the former, and long thin branches with smaller, coarsely articulated sclerites in the latter. I. minorbrachyblasta has bushy colonies with short densely packed branches, but considering both the lack of sampling for this taxon, documented panmixia and phenotypic plasticity of I. hippuris (e.g., Simpson 1906, Thomson \& Simpson 1909, Bayer \& Stefani 1987) in addition to the potential for parallel evolution throughout the I. hippuris distributional range relative to environmental clines, this latter taxonomic assignment is treated with extreme caution and may simply be an intermediate form. The two morphotypes of $I$. hippuris presented here have but partial adherence to those previously described. The shortbranched predominantly planar colonies at Ridge 1 are more akin to I. hippuris whereas the more open, long-branched colonies at Sampela resemble I. reticulata but with thick branches as opposed to thin. Swollen branch tips characteristic of I. hippuris were observed in both morphotypes is not a reliable trait. Such swollen branch tips were more prevalent at Sampela, which was the only site where external brooding was observed (Chapter 4; Figure 4.5), and therefore, swollen branch tips may pertain to the presence of eggs within the polyps.

\subsubsection{Isis hippuris phenotypic variability}

Measuring a broad range of phenotypic traits between I. hippuris morphotypes highlights trait integration, canalisation and thus those acted on by selection which may differ from those previously described for other gorgonian taxa (e.g., Sánchez \& Lasker 2003, Sánchez et al. 2003, 2004, 2007, Sánchez 2004, Dueñas \& Sánchez 2009). Here clear patterns of colony, therefore branching, integration coupled with sclerite-level traits and polyp density are consistent between the two morphotypes. Specifically, branching dynamics and colony size (colony porosity as a function of total branch number and size [projected branch area]) appear to have a negative association with sclerite size. Whether these traits are negatively associated as emergent properties or of longstanding, would necessitate further investigation using reciprocal transplant experimentation and population coalescence (Prada et al. 2008, Prada \& Hellberg 2013). In either case, differential light attenuation and nutrient components between the two sites are not unnatural phenomenon, which may or may not be exacerbated by the reef resource dependent anthropogenic influence from Sampela.

Colony surface area and metabolism are intrinsically linked whereby a cascade effect of concomitant variations in branching, polyp, canal and sclerite dynamics would be expected. However disintegration or canalisation was evident in both polyp and canal dimensions consistent with previous work (Sánchez 2004). Responses to variations in water quality, thus heterotrophic feeding capacity, are incurred through polyp density as opposed to size, yet both 
canal number and dimensions remained unchanged in both morphotypes. The exact function of stem canals is unclear (Cadena et al. 2011), although suggested to circulate and exchange water and nutrients throughout the coral colony (Ellis \& Solander 1786, Grillo et al. 1993, Gateño et al. 1998). Canalisation at this level further suggests that photosynthetic gain with nutrient translocation at the cellular level between endosymbionts and host, are the primary trophic resource. Optimal allocation theory posits an increase in the uptake of resource(s) that are most limiting growth (Bloom et al. 1985, Weiner 2004). Moreover, the same genotype can show resource allocation plasticity (Sebens 1997) in alternate environments consistent with the 'partitioning' hypothesis (Poorter \& Nagel 2000, Weiner 2004). Plasticity as a response is an emergent property of divergence (Schlichting \& Pigliucci 1998, Pigliucci 2005). Therefore to further elucidate energy allocation patterns between morphotypes, physiological tests coupled with morphological and genetic analyses on reciprocal transplants between reefs would establish phenotypic trait plasticity, thus plasticity capacity, or ecological divergence through disruptive selection (Schluter 1998, 2001) in I. hippuris.

Sclerite composition can vary relative to light and/or water motion (Muzik \& Wainwright 1977, West et al. 1993, Kim et al. 2004, Skoufas 2006, Clavico et al. 2007, Prada et al. 2008, 2013). Here the presence of numerous small, articulate interlocking sclerites could provide additional structural support for larger colonies found at Sampela, which lack the close branching structure present at Ridge 1. Smaller sclerites may mitigate mechanical constraints on the axis of increased colony size and bushy morphology through long thick branches, and provide greater soft tissue support as surface area increases (Clavico et al. 2007). Small clubs increase both flexion and torsion capacity in less exposed areas of Eunicella singularis Esper 1791 whereas larger spindles were prevalent in the exposed peripheral branches, yet Eunicella cavolinii Koch 1887 showed no selective difference (Skoufas 2006). A decrease in sclerite size with increased density in shallow conspecifics has been shown (e.g., Prada et al. 2008), typically due to increased water flow (West et al. 1993, West 1997, Brazeau \& Harvell 1994, Kim et al. 1997, 2004). Here, regardless of both morphotypes containing high densities of small sclerites, the consistency in small size at Sampela coupled with thicker longer branches and higher polyp density likely increases photosynthetic gain through greater surface area, as well as heterotrophic feeding. Canalisation due to a lack of variability in canal size or number, as well as polyp dimensions, suggests that photosynthetic gain from dinoflagellate endosymbionts is the primary resource for I. hippuris.

Sclerites are key characters for species identification yet susceptible to environmental 
perturbation and selection. All sclerites were consistently smaller in colonies from Sampela. The overall dimensions between the two morphotypes from both sites were within those described for I. hippuris with regards clubs and radiates from both I. reticulata and I. minorbrachyblasta. Sclerite differences between morphotypes compared to those published appeared inconclusive with notable crossover. For example, bent spindles characteristic of I. reticulata were present in colonies from Ridge 1, themselves bearing closer resemblance to I. hippuris. Interestingly, sclerite diversity was greater at Sampela with closer resemblance to I. reticulata, particularly considering sclerite asymmetry and crosses. No sclerites were found within the polyps or tentacles in either morphotype in this study, unlike in I. reticulata. However the small rods $(0.07$ x $0.01 \mathrm{~mm}$ ) of Bayer \& Stefani (1987) were present, but their precise location within I. hippuris soft tissue could not be determined, questioning their presence at all.

Enhanced fitness through an individual's (genotype) capacity to respond to environmental heterogeneity - specific morphotypes predominating in certain habitats - maximises survival through resource acquisition and minimises metabolic costs. Most corals are polymorphic under varying environmental conditions (West et al. 1993), with differential phenotypic expression of a genotype as a consequence of astogeny (colony development), itself genetically and/or environmentally mediated (De Rosa et al. 1999, Sánchez \& Lasker 2003, Garland \& Kelly 2006). Environmental influences on larval settlement, such as high sedimentation at Sampela or competition and high water flow at Ridge 1, may lead to developmental adaptational responses. Moreover, I. hippuris colonies survive and replicate through external brooding and asexual fragmentation with a propensity for philopatry and upward growth (Dauget 1992), increasing population structure and expansion on such degraded reefs over time.

Phenotypic divergence and biological success in I. hippuris within the WMNP may be a consequence of intraspecific polymorphism due to plasticity capacity with no barriers to gene flow between morphotypes. This, in part, can be a consequence of epigenetic effects, which may be heritable and become fixed through genetic assimilation if conditions persist. This, particularly in the presence of evolutionary capacitance whereby suppressed variation becomes functionally overwhelmed or initiated by the environment, can exert pleiotropic effects on poignant developmental processes (Rice 2008). Such non-additive genetic covariance wields a stronger influence on mutation than random drift, itself much stronger in small populations typical of brooding and asexual taxa. Thus, phenotypic divergence as seen in I. hippuris across sites within the WMNP, may be a consequence of hidden genetic variation leading to emergent environmentally mediated fixation accelerated by anthropogenic impact. Peripheral haplotypes 
reveal emergent lineages (Forsman 2003), those at Sampela up to seven base pairs differences between primary ITS2 sequence comparisons, none of which are shared with other haplotypes within the region. Furthermore, shared haplotypes and thus gene flow at the remaining test sites, with the exception of haplotype D (sea grass), suggests assortative mating with the onset of reproductive isolation at Sampela, the remaining haplotypes more frequent and broadly adapted, likely being ancestral. Greater sampling with genetic and coalescent analyses is required to confirm such supposition, particularly considering a minority presence of opposing morphotypes at each site (pers. obs.).

The consistent mutational differences both within (clones) and between sequenced samples, renders PCR or base calling errors unlikely. Given the renowned caveats associated with the ITS2 region such as intragenomic variation, secondary structure of each haplotype confirmed molecular morphometric differences most notably between Sampela (haplotype A) and the remaining sites, yet with strong sequence similarities between the remaining haplotypes. Furthermore, lack of network reticulation suggests no indication of hybridization, validating confidence in two species taxonomic assignment, emergent or previously diverged. However, hybridization at this juncture cannot be overlooked, I. hippuris morphotypes across its distributional range may also represent an Indo-Pacific syngameon as seen in the diverse and polymorphic scleractinian Acropora Oken 1815 (Ladner \& Palumbi 2012), with widespread gene flow through introgression (Vollmer \& Palumbi 2002, Palumbi et al. 2011, Ladner \& Palumbi 2012). However, any species delimitation within the Isis genus in addition to I. hippuris is necessary before further inference can be made.

It is clear that pertinent crossover exists between previously described Isis taxa and those present within the WMNP. It is tempting to conclude that I. hippuris is a single species with an extensive phenotypic and geographical range, or that only I. hippuris are present in the Wakatobi with other taxa within the genus elsewhere. Tolerant taxa tend to possess wide geographic distributions compared to those that are not (Calosi et al. 2010). However, the historically perceived panmixia of I. hippuris is likely more than a single species and not that of a complex when considering similar repetitive phenotypic trait differences across its distributional range. Previous alternative taxonomic assignments are therefore questionable, as the standard error of phenotypic variance would be greatly improved by assessing differences between I. hippuris morphotypes with increased specimen analyses from throughout its distributional range; a beneficial strategy when dealing with highly polymorphic taxa. Again, tests of coalescence on numerous independent highly polymorphic markers (SNPs; Ladner \& 
Palumbi 2012) would be required in order to fully elucidate convergent environment-bygenotypic effects in I. hippuris across its distributional range.

\subsubsection{Isididae polyphyly}

Phylogenetic analyses confirm haplotype differences as well as polyphyly within the Isididae; a phenomenon recently reported using the putative octocoral mitochondrial marker mshl (Watling et al. 2012). Even as far back as the earliest part of the last century, Kükenthal (1919) considered the Isididae to be polyphyletic; the subfamilies within as independent groups and the axis a "convergent phenomenon." Furthermore, I. hippuris, itself the type species of this family and the subfamily Isidinae, appears to have minimal phenotypic similarities to virtually all other isidid taxa with the exception of the axis, yet even this has been shown to be scleritic (consist of fused sclerites; Milne-Edwards \& Haime 1857, Kükenthal 1919, 1924, Bayer 1955, Watling et al. 2012, in prep., but see Nutting 1910c). Such evidence naturally brings into question the validity of I. hippuris in its current classification. Polyphyly within gorgonian groups across bathymetry is not unknown (McFadden et al. 2006). I. hippuris is the only shallow and zooxanthellate representative of the Isidinae and Isididae respectively, the remainder being characteristic of the deep ocean.

The scleritic composition of the I. hippuris axis further sets it apart from both the Isididae and the suborder Calcaxonians, these being more closely affiliated with the Alcyoniinan-Holaxonian clade as phylogenetically determined by Bernston et al. (2001) and McFadden et al. (2006). However this convergent trait holds significant evolutionary intrigue. The fused scleritic internodes with gorgonin nodes of the I. hippuris axis, ensures flexibility and durability in high water energy conditions. Yet what is the selective advantage of a jointed axis in deep-sea isidids? Empirically, this is undetermined but it is not unreasonable to propose that the jointed axis is a relictual anachronism consequential of geological (e.g., opening of the Drake Passage, Watling pers. comm.) as well as later glacio-eustatic sea-level changes resulting in bathymetric refugia from turbulent shallow coastal waters (Helm \& Schülke 2003). Thus, the functional significance of an articulated axis at depth is still a mystery; however longer internodes in the colonies at Sampela - like those seen in the benign deep ocean Isidids - compared to Ridge 1 were observed but not quantified (pers. obs.). Interestingly, deep-sea low flow specialists Isidella Gray 1857, have long elegant calcareous internodes compared to the larger much more robust internodes of Keratoisis Wright 1869, characteristic of moderate flow environments in the deep-sea (Watling pers. comm.), yet with no appreciable flexibility. A deep divergence with stabilizing selection regards a non-sclerite axis in deep-sea isidids may have occurred. Whether 
the I. hippuris axis is a consequence of convergent evolution based on ecological necessity in heterogeneous environments typical of shallow reefs or deep inheritance is unclear and under investigation (Watling et al. in prep.).

\subsection{CONCLUSION}

The two distinct I. hippuris morphotypes within the WMNP are phenotypically segregated through trait integration between healthy and degraded reefs, likely reinforced through reproductive strategy. The co-variability of light, sediment and water flow between sites fortify directional trait selection (Feder 1998); colony, branching dynamics, polyp density, sclerite size and diversity all vary significantly between sites. Moreover, polyp and nutrient canals appear canalized due to the additive effect of modules to the colony as opposed to an increase in size, raising inference to maximizing photosynthetic yield and heterotrophy, both mitigating and capitalizing on environmental conditions particularly at Sampela. Diverse phenotypic trait assessment through character trait integration using reciprocal transplant experiments across the two sites would undoubtedly be insightful, particularly as shifts in metabolic function are subject to selection at opposite ends of environmental gradients (Feder 1998). Selection acts on phenotypic variation (reflecting variation in gene expression), which may have become fixed over time leading to ecological divergence. I. hippuris morphotypes, tentatively confirmed by ITS2 sequences and secondary structure analyses, have only partial adherence to previously described taxa. It lacks prudence to assign species at this juncture necessitating integrative classical taxonomic, genomic, axis composition, biogeographical and ecological analyses across its distributional range. Furthermore, compelling phylogenetic evidence not only confirms $I$. hippuris morphotype differences, but also reveals its disassociation within the Isididae. Phylogenetic discernment investigating congruence between skeletal structure, multi-locus nextgeneration sequencing and coalescence modelling (Puritz \& Toonen 2011, Puritz et al. 2012), will assist unresolved hypotheses within this group. Thus is the continuum of evolution, compartmentalized for the necessity of biodiversity assessment and conservation management, itself a human construct against its own influence. 


\section{CHAPTER 4: ACCLIMATORY CAPACITY OF THE GORGONIAN ISIS HIPPURIS LINNAEUS 1758 TO ENVIRONMENTAL CHANGE IN SE SULAWESI, INDONESIA.}

\section{ABSTRACT}

Coral reefs within the Indonesian archipelago are some of the most exquisite yet anthropogenically compromised marine ecosystems. Within the Wakatobi Marine National Park (WMNP), SE Sulawesi, Indonesia, pronounced environmental clines are either caused or exacerbated by marine resource subsistence and destruction. The zooxanthellate gorgonian (sea fan coral) Isis hippuris Linnaeus 1758 however, thrives on degraded reefs, with distinct morphotypes across contrasting reef environments within the region. To further investigate whether the biological success of I. hippuris morphotypes are a consequence of selective mechanisms acting on phenotypic plasticity capacity or ecological divergence, reciprocal transplant experiments (RTEs) measuring multiple traits (morphological, physiological and environmental) were conducted across environmental gradients of light attenuation and anthropogenic disturbance. After one-year survivorship was lowest in clones transplanted from healthy to the degraded reef, suggesting the onset of immigrant inviability. Phenotypic traits were grouped into modules (colony, polyps, sclerites, optical parameters) and subject to duplicate multivariate models between the two sites. Significance values were consistently driven by differences between resident morphotype colonies from the healthy and impacted sites. The phenotypic traits in healthy source colonies consistently showed significant trait plasticity, whereas impacted residents were relatively insensitive to environmental change. Of the 38 phenotypic traits assessed, 17 were identified as driving test dissimilarities most notably in branching dynamics, polyp density, capstan and spindle sclerite dimensions, and Symbiodinium chlorophyll a light energy absorbance efficiency (for photosynthesis). Specifically, photoacclimatory responses were integrated at the morphological and bio-optical levels, with chlorophyll $a$ light harvesting efficiency maintained during reduced pigment density through an increase in host sclerite articulation actually maximizing the internal light field in healthy clones on degraded reefs. Variable optical responses were not however, attributed to endosymbiont type as all test colonies possessed a novel Symbiodinium Clade D1a. In summary, patterns of phenotypic variability within the I. hippuris holobiont likely represent incipient ecological divergence, with high plasticity capacity becoming fixed through ongoing anthropogenic disturbance on degraded reefs. 
Key words: Gorgonian coral · Plasticity capacity · Holobiont · Symbiodinium · Reciprocal transplants $\cdot$ Indonesia

\subsection{INTRODUCTION}

Environmental heterogeneity and perturbation may enhance or diminish biodiversity through differential species responses. High-energy biodiversity hotspots such as coral reefs within the Coral Triangle provide ideal environments for the investigation and interpretation of phenotypic variation within and between populations, particularly in response to anthropogenic disturbance. The biological success of such reef inhabitants may be a consequence of divergent selection or acclimatory capacity at the phenotypic level (Weiner 2004). Mechanisms of phenotypic variation can be extrinsic (e.g., substrate, light, temperature, sedimentation, competition, predation, and hydrodynamics), intrinsic (e.g., developmental, life history, physiological, or genetic), a combination, or interaction of the two. Fitness enhancement producing phenotypic novelty through such interactions may lead to ecological divergence either as a by-product or direct selection if conditions persist (Schluter 2001, Hatfield \& Schluter 1999). Thus, phenotypic variability, once considered an inconvenience (West-Eberhard 1989, DeWitt \& Scheiner 2004), is in fact, the raw material of evolutionary processes that maximizes survival at the individual and population level particularly in the face of environmental change.

Colonial sessile marine taxa are arguably the most pliable to changes in environmental regime through hierarchical modularity necessitated by their physical inability to relocate. Gorgonian corals (Cnidaria: Octocorallia) exhibit a diverse complexity, which is much greater and older than that of Scleractinia (Waggoner \& Collins 2004), yet they are poorly understood particularly throughout the Indonesian archipelago (van Ofwegen 2004). Within the WMNP, two distinct morphotypes of the zooxanthellate gorgonian Isis hippuris Linnaeus 1758 were found to be segregated between healthy and exploited reefs; short-branched multi/planar colonies and longbranched bushy colonies respectively (Chapters $2 \& 3$ ). Inferred integrated phenotypic traits at the colony, polyp and sclerite (skeletal element) levels were corroborated by haplotype differences, which suggest an emergent lineage on exploited reefs with remaining and likely ancestral haplotypes broadly distributed throughout the surrounding area. No indication of hybridization further reinforced the notion of two separate lineages whether emergent or previously diverged. Satisfactory taxonomic assignment to either morphotype however, was compromised by the historically recognised plasticity of I. hippuris (Wright \& Studer 1889, Simpson 1906, Thomson \& Simpson 1909, Bayer \& Stefani 1987, Fabricius \& Alderslade 2001) and tenuously described alternatives (Isis reticulata Nutting 1910, Isis minorbrachyblasta Zou, 
Huang \& Wang 1991). Furthermore, such phenotypic patterns have been repetitively documented in different regions (e.g., Philippines, Mai-Bao-Thu \& Domantay 1971, Bayer \& Stefani 1987; China, Zou et al. 1991; Okinawa, Muzik \& Rowley pers. obs.), which may suggest selective convergence indicative of parallel evolution (Schluter \& Nagel 1995). Thus, investigations into I. hippuris phenotypic segregation within the WMNP may act as a surrogate for determining the selective mechanisms that underlie the phenotypic differentiation recapitulated across its geographic range.

Concomitant phenotypic and genetic differentiation between I. hippuris morphotypes across two contrasting reef environments clearly indicates ecological divergence is at play. Yet both taxonomic ambiguity and likely divergence by gene flow obscures definitive species and ecological boundaries at the mechanistic level. Akin to terrestrial seed dispersal, allopatric barriers to gene flow in the aquatic realm are seldom applicable, with arguably greater reinforcement of disruptive selective mechanisms in sympatry. Ecological boundaries have been shown to be as powerful as they are numerous, specifically with the added dimension of human encroachment (Palumbi 2001, Puritz \& Toonen 2011). Therefore, I. hippuris on degraded reefs within the WMNP likely represent morphological stability that has come from phenotypic plasticity which has become fixed on degraded reefs, in other words, an environment-bygenotype effect that has become (or in the process of becoming) fixed over time in a degraded reef environment. Phenotypic divergence may have become a necessity to maintain survivorship on such reefs, with the cost of plasticity capacity greater than phenotypic stability over time. Therefore, time to divergence would be reflected in immigrant inviability (Prada \& Hellberg 2013) and further reinforced through assortative mating, particularly considering that I. hippuris has a propensity for philopatry coupled with replication through external brooding and asexual fragmentation leading to population structure and expansion on degraded reefs. Thus the eventual effect of directional and stabilizing components such as prolonged ecological disturbance, on disruptive selection between I. hippuris morphotypes could evolve through gene flow (Johnson 1976) within the Wakatobi.

Determining mechanisms of phenotypic variation provides valuable insight into the processes of divergence between morphotypes. Comparative measurements of I. hippuris phenotypic traits between contrasting environments over time are indicative of phenotypic expression patterns and can be visualised through the slope of a reaction norm (summarised in Chapter 1; Figure 1.5). In sum, trait fixation depicts environmental insensitivity and therefore no slope/change; trait plasticity depicts environmental sensitivity through a slope in the reaction norm. Phenotypic 
comparisons between morphotypes denote differential responses to environmental change, providing hypotheses for potential evolutionary mechanisms of selection (Pigliucci et al. 2006). However, whether trait plasticity is adaptive (genetic) or epigenetic (developmental) is undetermined (DeWitt \& Scheiner 2004, Schluter 2000). Environmentally induced change within and between I. hippuris morphotypes demonstrates plasticity capacity; but phenotypic changes in healthy reef morphotypes only, would demonstrate plastic and canalised phenotypes for healthy and degraded reefs respectively, therefore two different species as would be the case with no change visualised in either morphotype. Multivariate trait analyses engaging a "pluralistic approach" (Gould \& Lewontin 1979) would help discern mechanisms of evolutionary change within the holobiont and its environment. Yet it is surprising that studies on zooxanthellate gorgonians predominantly from the Caribbean, overlook the functional significance of their photosynthetic endosymbionts (West et al. 1993, Sánchez \& Lasker 2003, Kim et al. 2004, Sánchez 2004, Prada et al. 2008, Prada \& Hellberg 2013). Reciprocity between endosymbiont and host must surely bring about changes in the holobiont phenotype (Johnson 1976, Gilbert et al. 2010): the integrated whole being greater than the sum of its parts.

Zooxanthellate gorgonian corals typically show endosymbiont specificity (Goulet et al. 2008) with I. hippuris from the Great Barrier Reef known to harbour the putatively stress-tolerant clade D Symbiodinium (van Oppen et al. 2005). Technological advances reveal specificity gradients within endogenous Symbiodinium communities, irrespective of host - algal symbiosis assignment ('specificity' or 'flexibility'; Silverstein et al. 2012). Endogenous symbiont 'shuffling' between cryptic and dominant clades (A - I; Pochon \& Gates 2010), or types within clades in response to environmental perturbation, is quite logical in terms of community ecology. It is not unlikely that endosymbiont community shifts function as a mechanism of enhanced physiological performance, enabling holobiont persistence particularly on degraded reefs. Alternatively, endosymbiont communities can remain constant with greater acclimatory capacity (Bellantuono et al. 2012) as a consequence of heritable (Molinier et al. 2006) epigenetic effects (Chinnusamy et al. 2009). However, both the methods and molecular markers of Symbiodinium detection are controversial (e.g., Apprill \& Gates 2007, LaJeunesse \& Thornhill 2011, Stat et al. 2011, LaJeunesse et al. 2012, Pochon et al. 2012) with the highly variable internal transcribed spacer (ITS2) region detecting over 400 within clade rDNA 'types' (LaJeunesse 2002, 2005, LaJeunesse et al. 2003, 2004a, b), lending skepticism to its taxonomic efficacy (Stat et al. 2011). Nonetheless, the reported presence of Symbiodinium clade D within $I$. hippuris colonies may well contribute to its biological success on degraded reefs within the WMNP. Moreover, holobiont physiological plasticity is suggested to be of a greater fitness 
advantage regards mechanisms of resilience to environmental change, than the shifting or alteration of photosynthetic endosymbiont cladal type alone (Bellantuono et al. 2012).

The interplay between I. hippuris morphotypes at the morphological and physiological level through phenotypic trait integration would reveal mechanisms of physiological tolerance to environmental change. Primarily, photoacclimatory responses of the I. hippuris holobiont through the adjustment of optical and biophysical properties could maximize light harvesting and photosynthetic efficiency, key to its survival and biological success. Translocation of photosynthetically fixed carbon from Symbiodinium, as well as the conservation and recycling of essential nutrients such as nitrogen from the host, enables the holobiont to persist in nutrientpoor tropical waters, with concomitant calcium carbonate deposition by the symbionts (Muscatine \& Weis 1992). Variations in physiological photoacclimatory properties are indicative of holobiont responses but are not necessarily limited by Symbiodinium (Falkowski \& Dubinsky 1981), with photophysiological optima controlled at both the colony (Kim \& Lasker 1998, Enríquez et al. 2005, Shaish et al. 2006) and cellular levels (Kirk 1994). High light intensities can provoke increased branching and/or pigment concentrations within endosymbiont cells minimizing photoinhibition (irradiance damage to photosystem II [PSII]) through selfshading (Hoegh-Guldberg \& Jones 1999, Enríquez et al. 2005). Self-shading at the cellular level - a more rapid and possibly only self-shading response if colony morphology is fixed - can maintain relative zooxanthellar cell densities in fluctuating irradiances (Falkowski \& Dubinsky 1981, Porter et al. 1984, Dubinsky et al. 1990). However, acute environmental perturbation(s) such as increased nutrient levels, light and/or temperature can lead to marked endosymbiont proliferation (Muscatine et al. 1989, Dubinsky et al. 1984, 1990) or reduction (Wilkerson et al. 1988), particularly in non-acclimated colonies (e.g., Bellantuono et al. 2012). Still, trait mitigation of environmental susceptibility through prolonged exposure (Brown et al. 2000, 2002, Middlebrook et al. 2008, Bellantuono et al. 2012) may eventually become fixed (Molinier et al. 2006). Furthermore, utilization of the internal light field through skeletal light scattering reduces pigment investment and self-shading, whilst maintaining light-harvesting efficiency (Enríquez et al. 2005, Stambler \& Dubinsky 2005). Therefore, particular sclerite morphology and size may affect internal light reflection thereby facilitating and maximizing the harvesting of solar energy within I. hippuris morphotypes. Subsequent photosynthetic yield and hence productivity will therefore be dependent on integrative phenotypic mechanisms within the holobiont and should be considered in its entirety when assessing differential phenotypic responses of I. hippuris morphotypes to environmental change. 


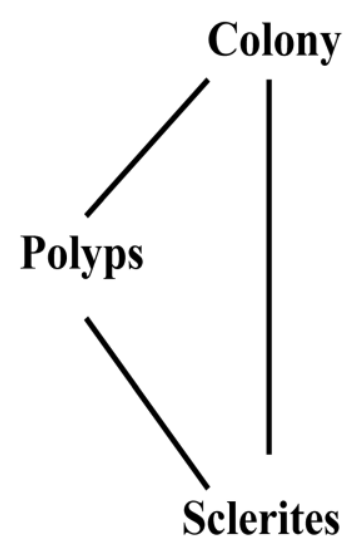

(a)

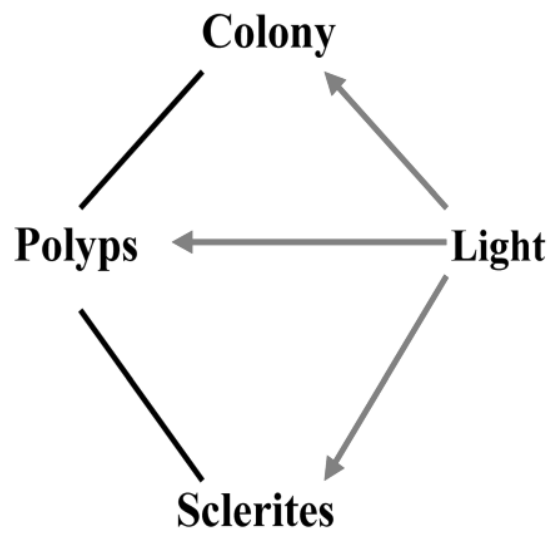

(b)

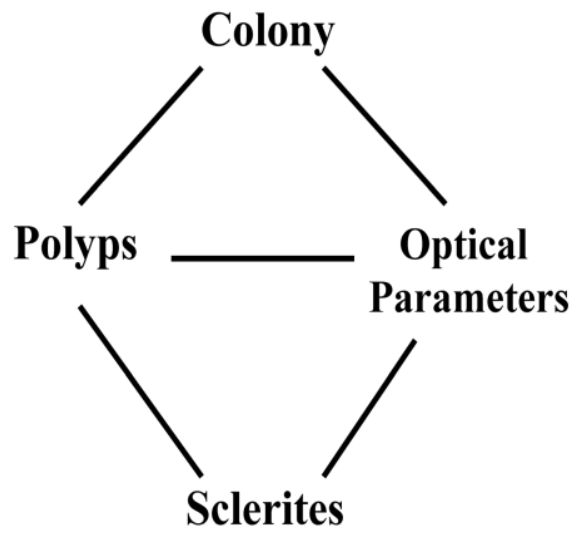

(c)

Figure 4.1 Isis hippuris (a) descriptive, (b) hypothesized causal and (c) proposed phenotypic module integration models.

Previous descriptive results segregate three phenotypic modules for I. hippuris morphotypes at opposing reef environments within the WMNP: colony, polyp, and sclerite level traits (Figure 4.1a; Chapter 3). The probability that any number of possible trait combinations can give rise to a novel phenotype, maximizing individual and/or population fitness in any one environment (Santelices 1999, Magwene 2001b) appears vast. However, multivariate selection recognises and delimits integrated traits, leading to tests of plasticity capacity or divergence through reaction norms as a product of reciprocal transplant experiments (RTEs). Here, it is hypothesized that light availability is a primary vector (causal; Figure 4.1b) of I. hippuris morphotypes between opposing sites (with distinct light regimes) within the WMNP, further driving integration among phenotypic traits. The quantum efficiency of the $I$. hippuris holobiont through the functional integration of optical traits (as a phenotypic module sensu lato; Figure 4.1c) was therefore assessed for evidence for the onset of light-induced directional selection or plasticity capacity. This was achieved through a one-year RTE measuring multiple traits (morphological, physiological, and environmental) between sites of contrasting reef health at comparable optical depths. Research objectives were as follows: (1) determine if I. hippuris morphotypes across environmental gradients are environmentally induced (plastic) or genetically derived (canalised/fixed); (2) assess differential physiological responses of the $I$. hippuris holobiont to environmental change; (3) investigate host algal endosymbiont specificity between morphotypes across and as a consequence of environmental change; (4) determine integrated phenotypic traits which interact to delimit I. hippuris morphotypes suggesting mechanisms of divergence through phenotypic trait integration in response to environmental perturbation. 


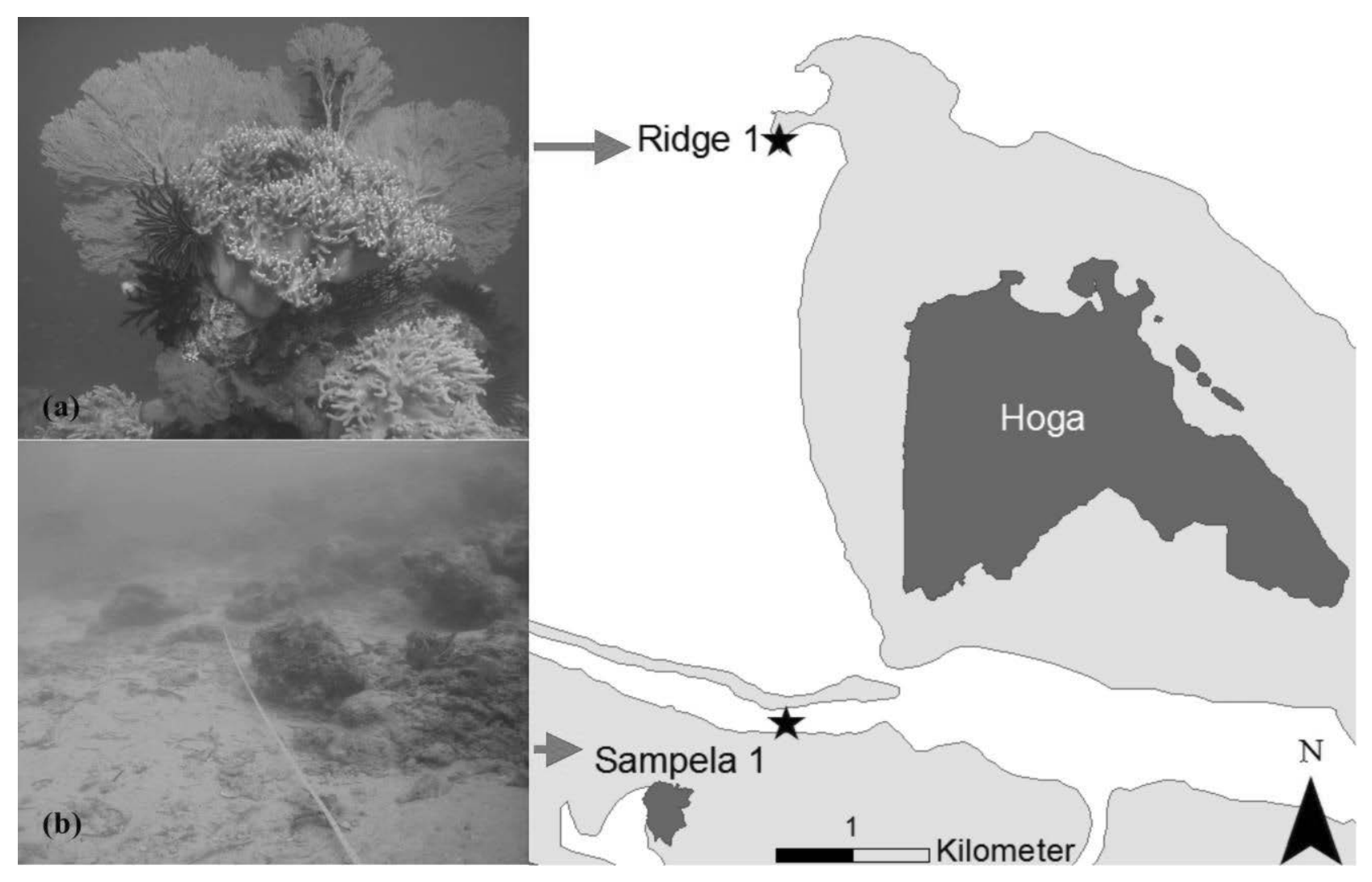

Figure 4.2. Comparative test site location map of (a) Ridge 1 and (b) Sampela off the islands of Hoga and Kaledupa respectively, within the WMNP, Indonesia.

\subsection{METHODS}

\subsubsection{Study Area}

The Wakatobi Marine National Park (WMNP) is the second largest national marine park in Indonesia (ca. 13,900 km²). Comprising ca. $600 \mathrm{~km}^{2}$ of the most biodiverse coral reefs centered within the Coral Triangle, the WMNP sustains a burgeoning human population of $>100,000$ people within S.E. Sulawesi supporting extensive marine resource reliance and subsequent destructive marine fisheries (Clifton et al. 2010, Clifton 2013). The dynamic coalescence between natural coral reef environments including the interplay between sea-grass beds, mangroves and human settlements renders the WMNP an ideal natural laboratory in which to study the effects of environmentally induced plasticity or divergence, particularly in the case of I. hippuris.

This research was conducted between two contrasting reef sites at opposite ends of a marked environmental gradient ca. $5 \mathrm{~km}$ apart (Figure 4.2) characterised by differential light attenuation, sedimentation and hydrodynamic regime (Table 4.1). Ridge 1 (healthy) is a high hydrodynamic, biodiverse offshore reef ridge with shear walls, strong deep-water nutrient rich upwellings and 
Table 4.1. Characteristic environmental variables at optical equivalent depths $(\zeta)$ between the two study sites in the WMNP, Indonesia.

\begin{tabular}{|c|c|c|c|c|}
\hline \multirow{3}{*}{ 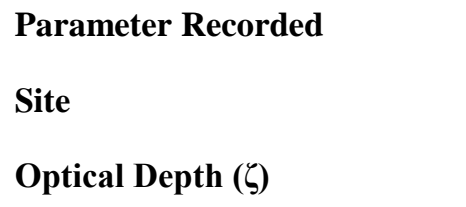 } & \multicolumn{4}{|c|}{ Mean value \pm SE (where appropriate) } \\
\hline & \multicolumn{2}{|c|}{ Sampela } & \multicolumn{2}{|c|}{ Ridge 1} \\
\hline & 2-3 m & $10 \mathrm{~m}$ & $5 \mathrm{~m}$ & $18 \mathrm{~m}$ \\
\hline Latitude, Longitude & \multicolumn{2}{|c|}{$005^{\circ} 29^{\prime} 01^{\prime \prime} \mathrm{S}, 005^{\circ} 26^{\prime} 57^{\prime \prime} \mathrm{E}$} & \multicolumn{2}{|c|}{$005^{\circ} 26^{\prime} 57^{\prime \prime} \mathrm{S}, 123^{\circ} 45^{\prime} 38^{\prime \prime} \mathrm{E}$} \\
\hline Temperature $\left({ }^{\circ} \mathrm{C} \min -\max \right)$ & $27.88-29.26$ & $25.61-28.09$ & $26.96-28.10$ & $24.06-28.07$ \\
\hline Light $\left(\mathrm{K}_{\mathrm{d}(\mathrm{PAR})} \min -\mathrm{max}\right)$ & $0.44-0.66$ & $2.21-2.65$ & $0.5-0.625$ & $2.25-2.5$ \\
\hline Flow $(\mathrm{cm} / \mathrm{s})$ & $0.86 \pm 0.19$ & $1.54 \pm 0.62$ & $34.38 \pm 1.33$ & $29.28 \pm 2.85$ \\
\hline Chlorophyll- $a\left(\mu \mathrm{g} 1^{-1}\right)$ & $0.38 \pm 0.001$ & $0.39 \pm 0.001$ & $0.30 \pm 0.001$ & $0.30 \pm 0.001$ \\
\hline Turbidity (NTU) & $4.73 \pm 0.72$ & $3.76 \pm 0.24$ & $0.15 \pm 0.43$ & $0.11 \pm 0.21$ \\
\hline Sedimentation $\left(\mathrm{g} \mathrm{d}^{-1}, \mathrm{n}=12\right)$ & $3.85 \pm 0.11$ & $3.22 \pm 0.10$ & $1.2 \pm 0.05$ & $1.14 \pm 0.05$ \\
\hline Sediment grain size $(\Phi, \mathrm{n}=12)$ & $2.5[125-250 \mu \mathrm{m}]$ & $5[31.25-62.5 \mu \mathrm{m}]$ & $1[0.5-1 \mathrm{~mm}]$ & $1[0.5-1 \mathrm{~mm}]$ \\
\hline
\end{tabular}

low turbidity. Sampela (impacted) is a low water flow, high sedimented, semi-lagoonal reef ca. $400 \mathrm{~m}$ distance from a sea gypsy (Bajo) village of ca. 1600, resulting in continual reef resource exploitation, destructive fisheries and wastewater exposure for at least 90 years (Webber H., pers. comm.). Additional sites within the area were not considered due to logistical constraints.

Characteristic environmental variables suggested to drive morphotype distribution at the two study sites (Chapter $2 \& 3$ ) were quantified (Table 4.1). Optical equivalent depths $\left(\zeta=K_{d(P A R)}\right)$ were calculated from the average $\mathrm{K}_{\mathrm{d}(400-700 \mathrm{~nm})}$ for source colony collection and transplant block deployment at the two study sites. Light $\mathrm{K}_{\mathrm{d}(\mathrm{PAR})}$ was taken every $1 \mathrm{~m}$ depth at 12:00 pm over consecutive days using the external photosynthetically active radiation (PAR) sensor on a Diving-Pulse Amplitude Modulation (Diving-PAM) fluorometer (Walz $\mathrm{GmbH}$ ), calibrated against a Li-Cor quantum sensor. The average site $\mathrm{K}_{\mathrm{d}}$ per optical equivalent test depth was calculated as described by Hennige et al. (2010) and is expressed in a reverse scale. Surface layer (i.e., recently settled) sedimentation rate was assessed using four replicate 1 litre sediment traps (English et al. 1997; Chapter 2) deployed for ca. 10 days each year at the calculated optical equivalent site depths. Grain size was estimated using Retsch Technology ${ }^{\circledR}$ test sieves, with logarithmically converted diameters expressed as phi $(\Phi)$ and classified using the Wentworth scale (Wentworth 1922). Additional suspended material was determined through turbidity (NTU as an inverse measure) and chlorophyll- $a$ (as $\mu \mathrm{g} \mathrm{L}^{-1}$ ) measurements, using $\mathrm{RBR}^{\circledR} \mathrm{XR}-420$ data loggers placed adjacent to each block repetitively throughout the duration of the experiment. Temperature $\left({ }^{\circ} \mathrm{C}\right)$ was determined using $\mathrm{HOBO}^{\circledR}$ data loggers placed on a single block at each depth per site, recording every $15 \mathrm{~min}$ throughout 

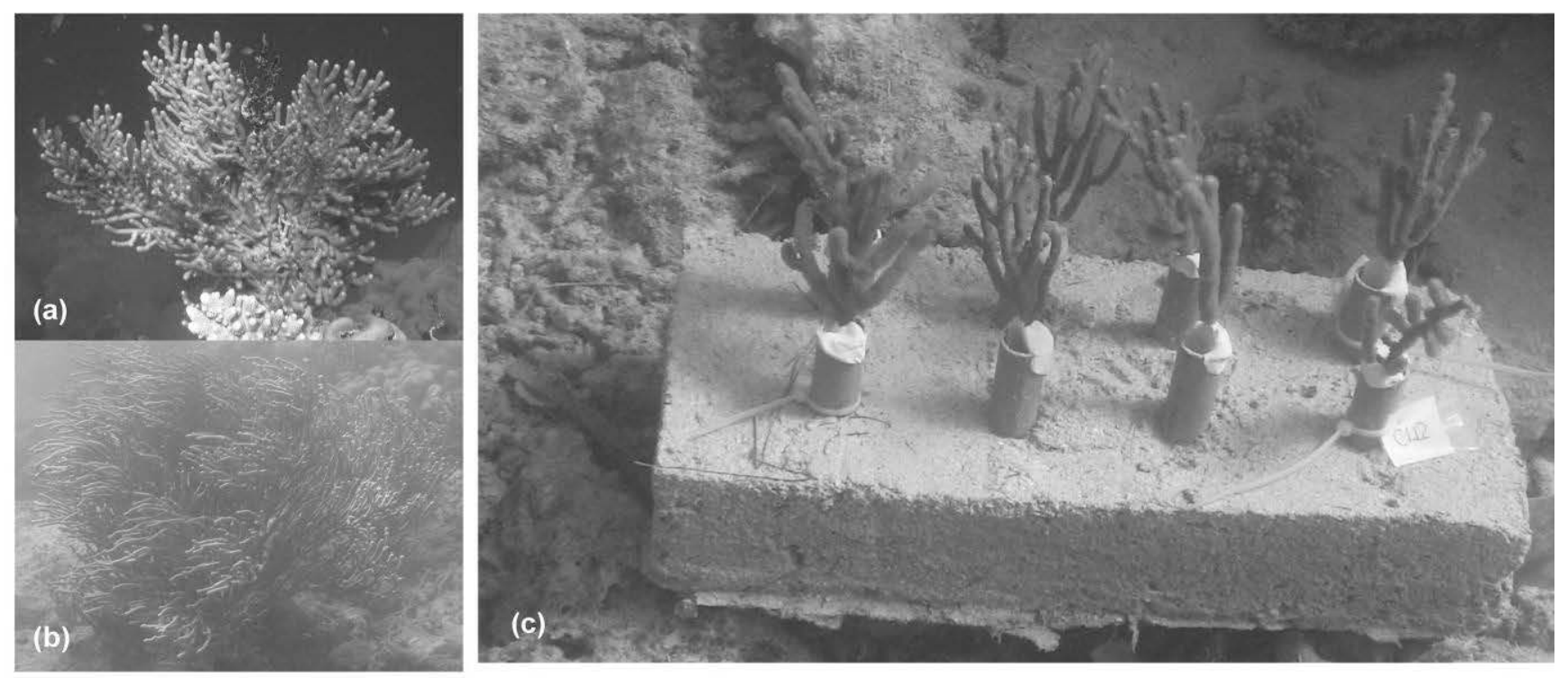

Sampela (Impacted)

Ridge 1 (Healthy)

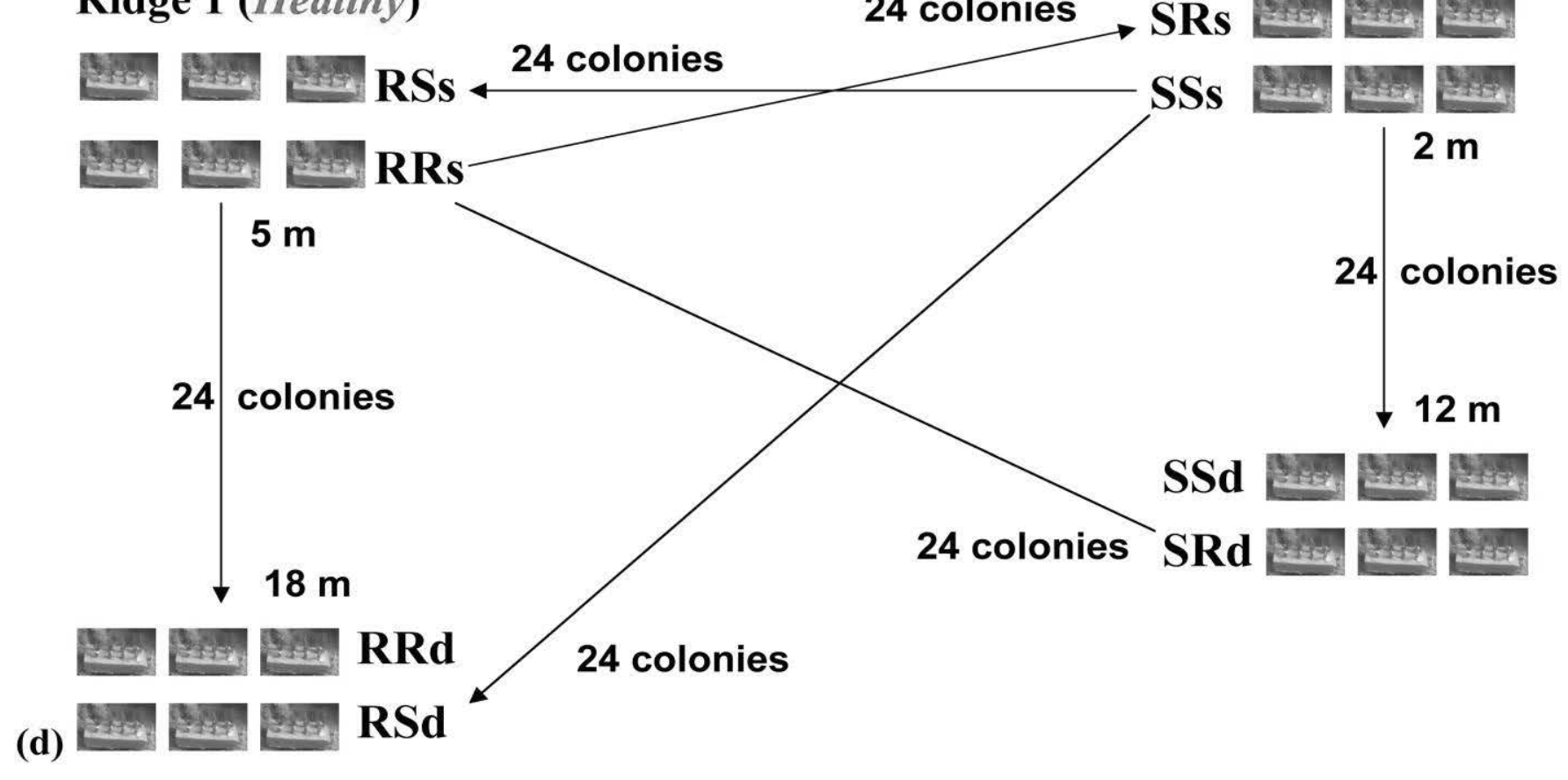

Figure. 4.3. Isis hippuris reciprocal transplant experimental design of source morphotypes from (a) Ridge 1, and (b) Sampela. (c) Transplant blocks baring eight clippings were placed (d) reciprocally with controls between optical equivalent $(\zeta)$ depths $5 \& 18 \mathrm{~m}$ at the Ridge, and $2 \&$ $12 \mathrm{~m}$ at Sampela. Arrows indicate direction of reciprocity and the number of transplanted test clippings. Transplant codes: RRs, Ridge - Ridge shallow; RSs, Sampela - Ridge shallow; RRd Ridge - Ridge deep; RSd, Sampela - Ridge deep; SSs, Sampela - Sampela shallow; SRs, Ridge - Sampela shallow; SSd, Sampela - Sampela deep; SRd, Ridge - Sampela deep. 
the test period. Water flow velocity was measured using a General Oceanics ${ }^{\circledR}$ flow meter with a low velocity rotor and custom made aluminum pipes for reef placement and expressed as $\mathrm{cm} \mathrm{s}^{-1}$. A hand-held GPS meter (GARMIN eTrex ${ }^{\circledR}$ ) determined latitude and longitude site coordinates which were not used in the statistical analyses.

\subsubsection{Field Experimentation}

I. hippuris test colony cuttings were reciprocally transplanted between the healthy reef Ridge 1 and impacted reef Sampela from June 2010 - July 2011 (Figure 4.3). Source colonies from each site $(n=24$; total $n=48)$ were selected at optical equivalent $\left(\zeta=K_{d(P A R)}\right)$ depths from the Ridge top $(5 \mathrm{~m})$ and Sampela reef crest $(3 \mathrm{~m}) \geq 10 \mathrm{~m}$ apart and of similar size to counter colony-level surplus resource variance. Scaled photographs were taken directly opposite and above each source colony using a Canon IXUS 900Ti, WP-DC7 u/w housing and INON UWL-105 AD x 0.51 lens. For each source colony, five $\sim 10 \mathrm{~cm}$ cuttings were sub-sampled, four for transplantation and one for comparative down stream analyses (see below). Scaled digital photographs were further taken of each cutting before and after transplantation for comparative annual growth and morphological measurements. A total of 24 cement blocks bearing eight colony cuttings (192 in total), secured using standard marine epoxy, were reciprocally transplanted between the two sites with additional blocks at optical equivalent depths as described above (Figure 4.3), to test for the effect of light on colony morphology, symbiont type, photobiology and stable isotope ratio (see below). Each block was placed such that transplant cuttings were perpendicular to the prevailing water flow.

All test colonies were sampled $(n=48$ in 2010, $n=192$ in 2011; total $n=240) 2 \mathrm{~cm}$ below the apex and preserved in $95 \% \mathrm{EtOH}, 70 \% \mathrm{EtOH}$ with prior overnight $4 \%$ formalin wash, and guanidinium solution for morphometric, taxonomic and zooxanthellar density, and genetic analyses respectively. Chlorophyll was immediately methanol extracted upon live sample recovery on site (see "Optical Assessments" section below and Enriquez et al. 2005).

\subsubsection{Comparative Phenotypic Traits}

To investigate potential mechanisms underlying distinct $I$. hippuris morphotypes, test (including controls) colony clippings between 2010 and 2011, were subject to a suite of phenotypic trait measurements with subsequent modelling for integrative traits. 

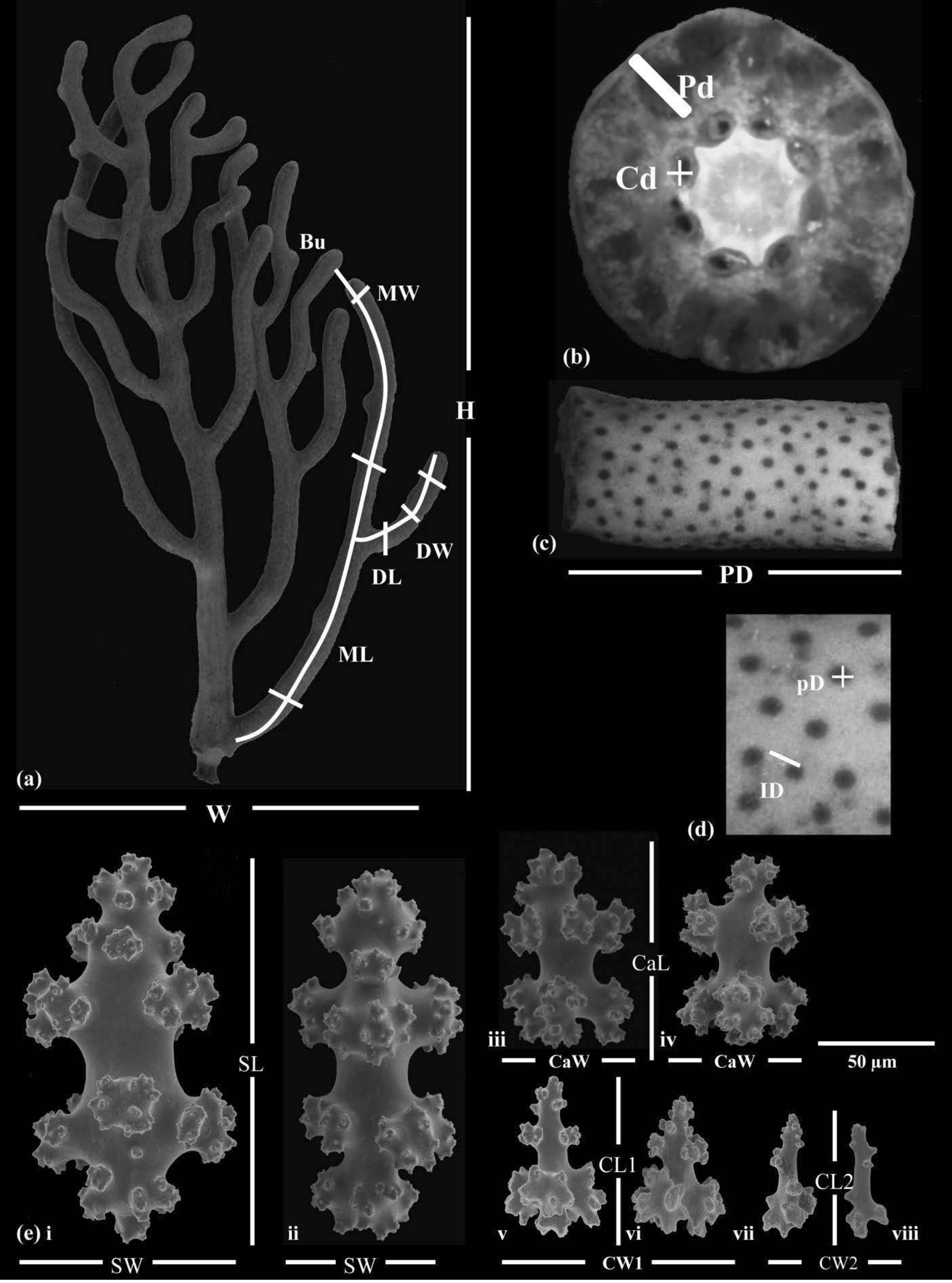
Figure 4.4. (Previous page) Isis hippuris morphological trait measurements of (a) test colony cuttings and branch dynamics; (b, c, d) canal and polyp dynamics; (e) sclerite site/morphotype comparisons of $\mathrm{i}$ and ii spindles, iii and iv capstan 7-radiates, v-viii clubs from Ridge 1 and Sampela respectively. Abbreviations as described in the above text and Tables 4.2 - 5 .

\section{Morphological Measurements}

Morphological trait comparisons were conducted on all I. hippuris test and control colony clippings, and divided into 14 macro-morphological (colony level) and 13 micro-morphological (polyp dynamics and sclerite level) traits (Figure 4.3, Table 4.3 - 5). Colony level measurements included colony height $[\mathrm{H}]$, and mean width [W] taken equidistant apart, colony base [B] and the projected colony area $[\mathrm{PA}]$ estimated by $\mathrm{H}$ x W. The PA was subsequently used to calculate colony porosity $[\mathrm{Po}]$ as a ratio of PA; the projected branch area [PBA] itself as the total branch length multiplied by the mean branch thickness (see below). Branch growth and development were further assessed using the hierarchical 'mother'/‘daughter' branch ordering system (Lasker et al. 2003, Sánchez \& Lasker 2003, Sánchez et al. 2003a). To correct for both temporal growth and positive association, thus size variation not related simply to growth, PA [incl. Po], PBA and TBL (see below) were also adjusted in equation (1) from Bayer et al. (1994):

e.g., PA $(\log )=(\log [$ final PA $]-\log [$ initial PA $]) / t$

where $t$ represents experimental duration in days. As growth persists nascent branches emerge (Bud\# [Bu]), daughter branches typically also become mother branches (e.g., second generation mother branch; see Figure 4.4) with the assumption of increased branch density over time. However, resource allocation change with growth would alter essential branching parameters such as [c; Sánchez 2004] defined as the ratio between the total branch number [TB\#] and total mother branch number $[\mathrm{MB \#}]$; and $[r]$ (Weinbauer \& Velimirov 1995) as the ramification rate defined as the annual growth difference between total branch length [TBL] and [TB\#]. Additional branching parameters were assessed via the quantification of daughter branch number [DB\#], mother branch length [ML], mean mother branch width [MW], daughter branch length [DL], and mean daughter branch width [DW]. Branch surface area was calculated on the geometric approximation of a cylinder from branch length and mean width as the radius, with subsequent polyp density [PD] per $\mathrm{cm}^{2}$. Twenty random measurements were taken per sample for inter-polyp distance [ID], and polyp diameter [pD] as the mean of two measurements (see Figure 4.4c). All polyp, branch cross-section and canal ([Cd] see Figure 4.4b) quantification were visualised under an Olympus SZX16 ${ }^{\circledR}$ stereomicroscope at 10x magnification with 0.5x 
objective.

Sclerites, typically the initial objects of selection (Bayer \& Stefani 1987), were quantified through the length and mean width of three measurements on 20 randomly selected sclerites per sclerite type; surface clubs [CL1/2, CW1/2]; and sub-surface capstans [7-radiates: CaL/W] and spindles $[\mathrm{SL} / \mathrm{W}]($ Figure $4.4 \mathrm{e}, \mathrm{i}$ - viii). Sclerites were removed by soft tissue dissolution in 5\% sodium hypochlorite solution and observed using optical microscopy (Olympus BX51 ${ }^{\circledR}$ ) and scanning electron microscopy (SEM) performed on a Hitachi S-800 SEM at the University of Hawai'i at Mānoa, USA. All micro-morphological measurements and sclerite preparation were taken $2 \mathrm{~cm}$ below the branch tip to avoid underdeveloped traits due to sub-apical branch growth (Lasker et al. 2003) and photographed using an Olympus 3.3MPX ${ }^{\mathrm{TM}}$ camera and Rincon software (ImagingPlanet ${ }^{\circledR}$ ). All macro- and micro-morphological characteristics were measured using ImageJ64 (Abràmoff et al. 2004).

\section{Optical Assessments}

Population dynamics of zooxanthellae within all test I. hippuris morphotypes were characterized from $\sim 1 \mathrm{~cm}$ fixed $\left(4 \% \mathrm{O} / \mathrm{N}\right.$ formalin wash and storage at $-40^{\circ} \mathrm{C}$ in $\left.70 \% \mathrm{EtOH}\right)$ host branches. Cells were isolated via repetitive $(3 \mathrm{x}$ at $4,000 \mathrm{~g})$ centrifugation-wash cycles (Muscatine et al. 1989) in filtered sea-water, and eight replicate $15 \mu \mathrm{L}$ aliquots of suspended cells per sample were enumerated using a haemocytometer. Zooxanthellar cell density [ZD] was normalized to coral surface area through the approximation of a cylinder as described above and expressed as cells $\mathrm{cm}^{-2}$. Cell division (cytokinesis) was qualified by a doublet appearance with observable plates and expressed as the percentage of total cells (mitotic index [MI], Wilkerson et al. 1988). Zooxanthellae mean diameter $[\mathrm{Zd}]$ and surface area to volume ratio $[\mathrm{SA}: \mathrm{V}]$ were measured using light microscopy at $200 \mathrm{x}$ magnification with an Olympus $3.3 \mathrm{MPX}^{\mathrm{TM}}$ camera and ImageJ64.

Optical density was determined using a U-3000 spectrophotometer with $\varphi-60$ integrating sphere (Hitachi) on live samples to assess chlorophyll- $a$ specific absorption [ $\left.a^{*}\right]$ (Enriquez et al. 2005), concentration $[A]$, and subsequent concentration per cell $[\mathrm{CZ}]$, testing the "packaging effect" or "self-shading" (Dubinsky et al. 1986, Kirk 1994). Live colonies were measured in triplicate with absorbance $D$ values taken from a single peak at $675 \mathrm{~nm}$ to minimize accessory host and algal pigment interference, whereby:

$a^{*}{ }_{\text {chla }}=(D / p) \ln 10$ 
with $D$ as the spectrophotometry absorbance via reflectance measurements for absorptance $[A]$, $p$ is the pigment content per unit branch surface area expressed as $\mathrm{mg} \mathrm{m}^{-2}$ (Enríquez \& SandJensen 2003, Enríquez et al. 2005, Hennige et al. 2010). Bleached (24 h chloride emersion) skeletal elements (axis and sclerites) were further measured as described by Enríquez et al. (2005) and the corresponding live soft tissue (ca. $1 \mathrm{~cm}^{2}$ ) was weighed, measured and dissolved in $5 \mathrm{~mL}$ of $100 \%$ methanol (Porra et al. 1989, Ritchie 2006) for $36 \mathrm{~h}$ at $4^{\circ} \mathrm{C}$ in darkness. Chlorophyll- $a$ concentration was then calculated from the dichroic equation:

$$
[A]=13.6849 \mathrm{E}_{665}-3.4551 \mathrm{E}_{632} \mu \mathrm{g} \mathrm{mL} L^{-1}
$$

where $E_{\lambda}$ is the extinction coefficient of light (photon) absorption at the given wavelength. All optical values were normalised to branch surface area, and chlorophyll- $a$ content per cell expressed as pg cell ${ }^{-1}$.

Prior to sub-sampling, light adapted (open photosystem II reaction centres) maximal quantum yield $(\Delta \mathrm{F} / \mathrm{Fm}$ ') of PSII (photosystem II) was measured using a Diving-PAM fluorometer set at measuring light intensity $=8$; actinic light factor $=0.5$; saturation pulse width $=0.8$; saturation intensity $=3$; gain $=3$; and signal damping $=2$. Distance between the coral branch surface tissue and the fiber optic probe was standardized $(10 \mathrm{~mm})$ using a DIVING-SH Walz surface holder. Light adapted yield ( $\Delta \mathrm{F} / \mathrm{Fm}$ ') was assessed averaging measurements taken in triplicate from the top ( $2 \mathrm{~cm}$ from the branch tip), middle, and base of each colony parallel to the coral-water interface. This was replicated on transplant retrieval in 2011, where measurements were taken 2 $\mathrm{cm}$ (top), $6 \mathrm{~cm}$ (middle), and $10-12 \mathrm{~cm}$ (base) from the apex of the colony. In both years quantum yield was consistently taken at 07:20 h \pm 10 min over eight consecutive days.

\section{Symbiodinium Genetics Analyses}

Genomic DNA of endosymbiotic dinoflagellates was extracted from all test colony clippings (n $=48$ in 2010, $\mathrm{n}=192$ in 2011; total $\mathrm{n}=240$ ) using a Guanidinium procedure as previously described (Pochon et al. 2001, Chapter 3). Three molecular markers were used to test for Symbiodinium diversity and marker utility. The relatively conservative mitochondrial-encoded cytochrome oxidase 1 (COX1_F2; forward; 5'-AAA TTG TAA TCA TAA ACG CTT AGG-3' and reverse COX1_R1; 5'-GGC ATA ACA TTA AAT CCT AAG AA-3') was used for all samples $(n=240)$. For a deeper diversity and phylogenetic assessment clone libraries were constructed using the ITS-LSU region including the 3'-end of the 5.8S (SSU) region, the entire internal transcribed spacer (ITS2) region to the 5 '-end of the LSU rDNA region (itsD forward; 
5'- GTG AAT TGC AGA ACT CCG TG-3' and $L O$ reverse; 5'- GCT ATC CTG AGR GAA ACT TCG -3'), and the plastid-coding psbA minicircle ( $p s b A$ Clade D specific primers; psbAFor_1; 5'-GCA GCT CAT GGT TAT TTT GGT AGA C-3' and psbARev_1; 5'-AAT TCC CAT TCT CTA CCC ATC C-3'; LaJeunesse \& Thornhill 2011) were used on a subset of test samples ( $n=24$ and $n=8$ respectively). PCR amplifications for each marker were conducted according to the following conditions, with a product volume of $50 \mu \mathrm{L}$ constituting: $5.0 \mu \mathrm{L}$ of 10x PCR Buffer (Bioline), $2.0 \mu \mathrm{L}$ of $\mathrm{MgCl}_{2}(2 \mathrm{mM}), 1 \mu \mathrm{L}$ of each primer $(10 \mathrm{mM}), 1 \mu \mathrm{L}(2.5$ $\mathrm{mM}$ of each dATP, dCTP, dGTP, and dTTP), $0.2 \mu \mathrm{L}$ of Hotstart Immolase Taq polymerase (Bioline Incl., London, UK), $1 \mu \mathrm{L}$ of DNA, and $39 \mu \mathrm{L}$ of sterile water. PCR amplification for COI and $p s b A^{n c r}$ initiated at $95^{\circ} \mathrm{C}$ for $10 \mathrm{~min}$, followed by 40 cycles at $94^{\circ} \mathrm{C}$ then $35 \mathrm{~s}$ at annealing temperatures of $55^{\circ} \mathrm{C}$ and $56^{\circ} \mathrm{C}$ respectively, $1.3 \mathrm{~min}$ at $72^{\circ} \mathrm{C}$, with a final extension at $72^{\circ} \mathrm{C}$ for $10 \mathrm{~min}$. Touchdown amplification was conducted for ITS-LSU as follows: denaturation at $95^{\circ} \mathrm{C}$ for $10 \mathrm{~min}, 25$ cycles at $94^{\circ} \mathrm{C}$ then $35 \mathrm{~s}$ at $65^{\circ} \mathrm{C}$ (reduction in annealing temperature of $0.5^{\circ} \mathrm{C}$ per cycle), and $2 \mathrm{~min}$ at $72^{\circ} \mathrm{C}$. A further 14 cycles of $30 \mathrm{~s}$ at $94^{\circ} \mathrm{C}, 35 \mathrm{~s}$ at $52^{\circ} \mathrm{C}, 2 \mathrm{~min}$ at $72^{\circ} \mathrm{C}$, and a final $10 \mathrm{~min}$ extension at $72^{\circ} \mathrm{C}$. Purified ITS-LSU and $p s b A$ products were ligated into the pGEM $^{\circledR}-\mathrm{T}$ Easy vector ${ }^{\mathrm{TM}}$ (Promega), transformed into $\alpha$ - Select Gold Efficiency ${ }^{\mathrm{TM}}$ competent cells (Bioline), with subsequent positive inserts verified by PCR using plasmid specific primers (M13). All sequences including clone library positive inserts (8 - 12 per library), were purified with an ExoSAP-IT kit, sequenced in both directions using the ABI Prism Big Dye ${ }^{\mathrm{TM}}$ Terminator Cycle Sequencing Ready Reaction Kit and run on an ABI 3100 Genetic Analyzer (Perkin-Elmer Applied Biosystems, Foster City, CA, USA) at the University of Hawai'i at Mānoa, USA. All genomic DNA and PCR amplicons were visualized on 1\% agarose gel.

Sequences were aligned using ClustalW2 (Thompson et al. 2002) and manually edited in Geneious Pro v.5.6.2 (Biomatters Ltd., NZ). A selection criterion of identical sequences from two or more clone libraries from independent samples was established for both ITS-LSU and psbA to minimize the effect of intragenomic variation and/or PCR artifacts on downstream analyses. All single variants were reverted to the library consensus. Phylogenetic reconstructions with comparative in house and GenBank sequences (see Table 4.2) were conducted using plugins within Geneious. 
Table 4.2 Accessions of comparative sequences of Symbiodinium clades used in the phylogenetic analyses. Asterisks indicate the COX1 outgroup.

\begin{tabular}{llll}
\hline Marker & Symbiodinium Clade & GenBank & Reference \\
& & & \\
\hline COX1 & All Clades & AY289689-AY289712 & Takishita et al. 2004 \\
COX1 & *Gymnodinium simplex & CCMP419 & Santos et al. 2002 \\
ITS & D & KC597691 & Padilla-Gamino et al. Unpub. \\
$I T S$ & D & AF396631 & Santos et al. 2003 \\
ITS & D1a & JN558076 & Pochon et al. 2012 \\
$I T S$ & D1a & AJ311948 & Pochon et al. 2001 \\
$I T S$ & D1a & AJ308900 & Pochon et al. 2001 \\
$I T S$ & D1a & JN558080 & Pochon et al. 2012 \\
$I T S$ & D1a & EU074897 & Thornhill et al. 2007 \\
$I T S$ & D2 & AF396627 & Pochon et al. 2006 \\
$p s b A$ & D & AB086877 & Takishita et al. 2003 \\
$p s b A$ & D & AB086878 & Takishita et al. 2003 \\
$p s b A$ & D & AB086863 & Takishita et al. 2003 \\
$p s b A$ & D & JQ043586 & LaJeunesse \& Thornhill 2011 \\
& & & \\
\hline
\end{tabular}

Phylogenetic inferences for the markers ITS-LSU and $p s b A$ were constructed using the neighbour joining (NJ) (Jukes \& Cantor 1969) and maximum likelihood (ML) (PHYML 2.1.0; Guindon \& Gascuel 2003) methods with 1000 bootstrap replicates (Felsenstein, 1985) and unrooted circle trees constructed with the latter (ML). Phylogenetic inference for COXI was constructed using MrBayes 2.0.5 (Huelsenbeck \& Ronquist 2001) in addition to NJ and ML, rooted with the dinoflagellate Gymnodinium simplex (Lohmann) Kofoid \& Swezy 1921. Maximum likelihood (ML) phylogeny was conducted using the best-fit model (JC) of nucleotide substitution as selected in jModelTest 2 (Darriba et al. 2012) using the Akaike Information Criterion (AIC). Bayesian inference (BI) phylogeny was made with a JC69 substitution model and burn-in of 100,000.

\section{Multivariate Analyses of Integration}

Investigations of trait integration were conducted using routines within the PRIMER-E v6.1.12 statistical package (Clarke \& Gorley 2006), with PERMANOVA+ v1.02 extension (Anderson 2001). To identify phenotypic trait integration on a functional level as a consequence of developmental $\left(V_{E}\right)$, environmental $\left(V_{G}\right)$ or an interaction of the two $\left(V_{G x E}\right)$ processes, an initial multivariate correlative $(p<0.05)$ approach was conducted as a Draftsman plot. Trait 'subsets' 

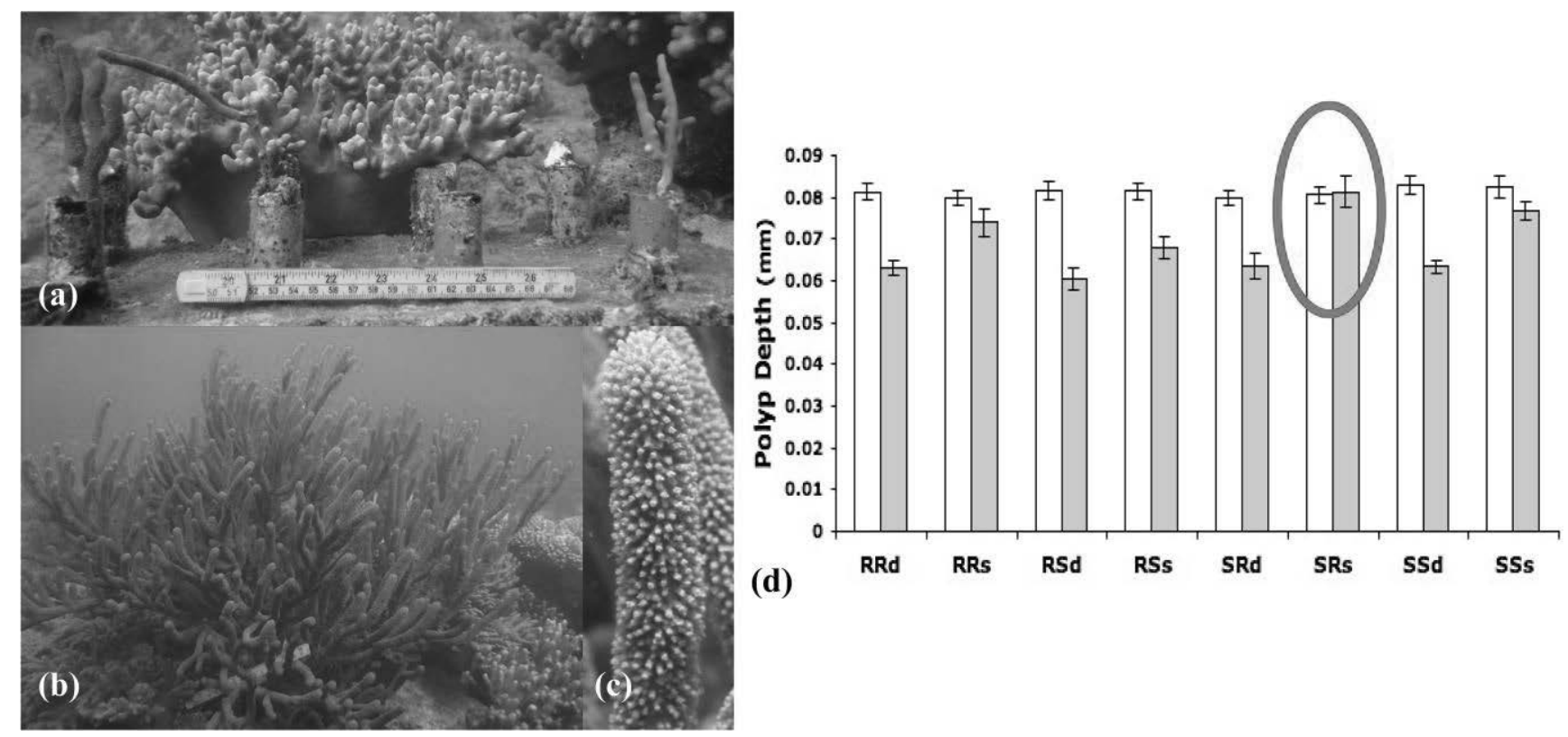

Figure 4.5. Isis hippuris colonies of (a) damaged transplant block clippings from Ridge 1 to Sampela at $3 \mathrm{~m}$ depth, (b) an adjacent resident, and (c) a close up of large pink externally brooded eggs and extended yellow polyps. (d) Mean ( \pm SE) polyp depth $(\mathrm{mm})$ from test colonies between 2010 (white bars) and 2011 (grey bars). Red ring highlights no change in polyp depth in transplanted colonies compared to all other test colonies.

or 'phenotypic modules' (Magwene 2001b) were subsequently selected on the basis of conditionality through correlation for downstream analyses. Reaction norms were constructed for all phenotypic traits tested and partitioned into the aforementioned phenotypic modules: colony, polyp, sclerite and optical dynamics. Phenotypic trait data were log transformed (correcting for positive association) and 'zero-adjusted' Bray-Curtis resemblance matrices constructed (Clarke et al. 2006b) for tests of plasticity vs. adaptation between and within the two study sites: Ridge 1 and Sampela. Sites were segregated by clone origin and multi-factorial models with 9999 permutations (PERMANOVA; Anderson 2001) were performed with reduced trait dimensionality visualised in the constrained canonical analysis of principal coordinates (CAP; Anderson and Willis 2003). Topological equivalent traits were identified using similarity percentages (SIMPER; Clarke 1993) and displayed as a vector overlay on the CAP ordination. Specific traits within and between delimited subsets were then modelled for phenotypic integration primarily in response to irradiance; environmental influence was investigated using nonparametric multivariate regression (McArdle \& Anderson 2001) with the DISTLMforward routine (Anderson 2003). Based on a Euclidean distance matrix, all raw environmental variables (Table 4.1) were normalised and significance tested using 9999 permutations (Anderson 2001). 
Table 4.3. Isis hippuris test colony annual mortality and survival. Values calculated out of 24 test clippings per treatment. Asterisk indicates likely reduction in test colony numbers due to a human disturbance event; revised values out of 16 in brackets.

\begin{tabular}{|c|c|c|c|}
\hline \multirow{2}{*}{ Treatment } & \multicolumn{2}{|c|}{ Mortality } & \multirow{2}{*}{ Survivorship rate } \\
\hline & $\mathbf{n}$ & $\%$ & \\
\hline \multicolumn{4}{|l|}{ Ridge (Source) } \\
\hline [RRs] Ridge - Ridge shallow & 1 & 4.2 & 0.958 \\
\hline [RRd] Ridge - Ridge deep & 0 & 0 & 1.000 \\
\hline [SRs] Ridge - Sampela shallow* & $11(6)$ & $45.8(37.5)$ & $0.583(0.625)$ \\
\hline [SRd] Ridge - Sampela deep & 2 & 8.3 & 0.917 \\
\hline \multicolumn{4}{|l|}{ Sampela (Source) } \\
\hline [SSs] Sampela - Sampela shallow & 3 & 12.5 & 0.875 \\
\hline [SSd] Sampela - Sampela deep & 2 & 8.3 & 0.917 \\
\hline [RSs] Sampela - Ridge shallow & 2 & 8.3 & 0.917 \\
\hline [RSd] Sampela - Ridge deep & 1 & 4.2 & 0.958 \\
\hline
\end{tabular}

All variables, with the exception of latitude and longitude, were entered into the statistical models as raw values. Values were edited visually with significant outliers removed.

\subsection{RESULTS}

\subsubsection{Comparative Phenotypic Traits}

A total of 38 phenotypic traits were assessed on all test colonies; 17 at the colony level, 5 at the polyp and canal level, 8 at the sclerite level, and 8 optical parameters including chlorophyll fluorescence (Tables 4.4 - 7). Of the 192 test colony clippings transplanted, 171 were recovered in 2011 and standardized to 5 colonies per test block for all subsequent trait assessments. Overall 431,761 phenotypic measurements/counts were made (2010 \& 2011). Survivorship between the two sites was considerable with $86.5 \%$ and $90.6 \%$ for Ridge and Sampela clones respectively and no signs of bleaching or predation observed. Only a single block (Sampela shallow transplanted from the Ridge, Figure 4.5) was damaged due to accidental human interference such that it was excluded from further analyses. The greatest test colony mortality was at Sampela (Table 4.3) specifically those transplants from Ridge 1 (SRs), which even with the exclusion of the damaged block, still had the lowest survivorship across all tests. Nevertheless, surviving colonies appeared healthy, probably by being elevated from reef competition for 12 months. The bivalve Pteria cf. tortirostris Dunder 1848 often associated with gorgonians, was present on shallow (SSs =2; SRs = 2) and deep $(\mathrm{SSd}=2 ; \mathrm{SRd}=6)$ clippings at Sampela and a single observation at Ridge $(\mathrm{RSd}=1)$. 
Table 4.4. Isis hippuris macro-morphological (colony level) traits of reciprocal transplant and control colony clippings sourced from Ridge 1. Test codes defined in Figure 4.2.

\begin{tabular}{|c|c|c|c|c|c|c|c|c|}
\hline \multirow{2}{*}{ Phenotypic Trait } & \multicolumn{8}{|c|}{ Ridge $1($ mean \pm SE) } \\
\hline & $\operatorname{RRs}(10)$ & $\operatorname{RRs}(11)$ & $\operatorname{RRd}(10)$ & $\operatorname{RRd}(11)$ & $\operatorname{SRs}(10)$ & SRs(11) & SRd(10) & SRd(11) \\
\hline \multicolumn{9}{|l|}{ Clipping (cm) } \\
\hline$[\mathrm{H}]$ Height & $10.62 \pm 0.45$ & $13.45 \pm 0.59$ & $10.25 \pm 0.41$ & $13.36 \pm 0.7$ & $10.12 \pm 0.36$ & $12.50 \pm 0.48$ & $11.48 \pm 0.47$ & $13.67 \pm 0.54$ \\
\hline [W] Width & $3.03 \pm 0.25$ & $4.98 \pm 0.43$ & $2.61 \pm 0.20$ & $4.20 \pm 0.35$ & $2.79 \pm 0.30$ & $4.26 \pm 0.40$ & $3.06 \pm 0.25$ & $3.75 \pm 0.30$ \\
\hline [B] Base & $0.55 \pm 0.03$ & $0.78 \pm 0.09$ & $0.54 \pm 0.03$ & $0.61 \pm 0.04$ & $0.60 \pm 0.05$ & $0.86 \pm 0.10$ & $0.57 \pm 0.03$ & $0.64 \pm 0.05$ \\
\hline [TBL] Total Branch Length & $51.83 \pm 4.24$ & $76.55 \pm 6.6$ & $46.35 \pm 3.11$ & $65.49 \pm 4.7$ & $48.47 \pm 4.58$ & $79.60 \pm 6.25$ & $47.28 \pm 2.97$ & $59.89 \pm 4.83$ \\
\hline [ML] Mother Length & $8.20 \pm 0.43$ & $9.96 \pm 0.49$ & $7.90 \pm 0.42$ & $9.88 \pm 0.62$ & $8.06 \pm 0.43$ & $9.40 \pm 0.60$ & $8.59 \pm 0.53$ & $9.90 \pm 0.64$ \\
\hline [MW] Mother Width & $1.18 \pm 0.17$ & $1.82 \pm 0.09$ & $1.03 \pm 0.15$ & $1.28 \pm 0.16$ & $1.09 \pm 0.16$ & $1.72 \pm 0.12$ & $1.14 \pm 0.14$ & $1.42 \pm 0.17$ \\
\hline [DL] Daughter Length & $2.85 \pm 0.17$ & $3.84 \pm 0.21$ & $3.17 \pm 0.17$ & $3.86 \pm 0.20$ & $3.26 \pm 0.22$ & $4.14 \pm 0.31$ & $2.83 \pm 0.17$ & $3.44 \pm 0.18$ \\
\hline [DW] Daughter Width & $0.34 \pm 0.02$ & $0.38 \pm 0.017$ & $0.35 \pm 0.03$ & $0.33 \pm 0.01$ & $0.33 \pm 0.02$ & $0.38 \pm 0.03$ & $0.35 \pm 0.02$ & $0.35 \pm 0.01$ \\
\hline$[\mathrm{Bu}] \mathrm{Bud} \#$ & $3.27 \pm 0.6$ & $4.13 \pm 0.55$ & $3.07 \pm 0.64$ & $4.33 \pm 0.53$ & $2.58 \pm 0.65$ & $3.8 \pm 0.52$ & $3.33 \pm 0.59$ & $3.0 \pm 0.58$ \\
\hline [TB\#] Total Branch \# & $13.67 \pm 1.48$ & $18.6 \pm 1.54$ & $12.13 \pm 0.91$ & $16.07 \pm 1.24$ & $12.5 \pm 1.45$ & $16.67 \pm 1.72$ & $12.67 \pm 1.15$ & $13.67 \pm 1.17$ \\
\hline [DB\#] Total Daughter Branch \# & $10.53 \pm 1.16$ & $14.47 \pm 1.3$ & $9.27 \pm 0.68$ & $12.47 \pm 0.86$ & $9.58 \pm 1.10$ & $13.1 \pm 0.8$ & $9.80 \pm 0.85$ & $10.6 \pm 0.84$ \\
\hline [MB\#] Total Mother Branch \# & $3.13 \pm 0.38$ & $4.13 \pm 0.42$ & $2.87 \pm 0.40$ & $3.60 \pm 0.51$ & $2.92 \pm 0.43$ & $3.56 \pm 0.36$ & $2.87 \pm 0.34$ & $3.07 \pm 0.37$ \\
\hline$[c] \mathrm{MB} \#: \mathrm{TB} \#$ & $4.76 \pm 0.38$ & $4.69 \pm 0.32$ & $5.15 \pm 0.60$ & $5.60 \pm 0.77$ & $4.72 \pm 0.40$ & $4.75 \pm 0.34$ & $4.85 \pm 0.41$ & $4.93 \pm 0.40$ \\
\hline$[r]$ Ramification rate & \multicolumn{2}{|c|}{$0.268 \pm 0.117$} & \multicolumn{2}{|c|}{$0.258 \pm 0.060$} & \multicolumn{2}{|c|}{$0.205 \pm 0.060$} & \multicolumn{2}{|c|}{$0.230 \pm 0.109$} \\
\hline [PA] Projected Area & $32.24 \pm 3.04$ & $67.34 \pm 6.97$ & $27.11 \pm 2.62$ & $57.0 \pm 6.68$ & $28.46 \pm 3.5$ & $53.05 \pm 5.21$ & $35.11 \pm 3.14$ & $51.71 \pm 4.84$ \\
\hline [PBA] Projected Branch Area & $43.03 \pm 7.09$ & $86.98 \pm 10.24$ & $34.0 \pm 5.48$ & $56.15 \pm 8.65$ & $38.09 \pm 7.10$ & $88.69 \pm 9.08$ & $38.16 \pm 5.2$ & $57.29 \pm 8.31$ \\
\hline [Po] Porosity \% & $87.02 \pm 0.26$ & $86.70 \pm 0.12$ & $87.03 \pm 0.26$ & $89.82 \pm 0.80$ & $87.03 \pm 0.26$ & $83.64 \pm 0.18$ & $87.03 \pm 0.26$ & $89.33 \pm 0.12$ \\
\hline
\end{tabular}


Table 4.5. Isis hippuris macro-morphological (colony level) traits of reciprocal transplant and control colony clippings sourced from Sampela. Test codes defined in Figure 4.2.

\begin{tabular}{|c|c|c|c|c|c|c|c|c|}
\hline \multirow{2}{*}{ Phenotypic Trait } & \multicolumn{8}{|c|}{ Sampela $($ mean \pm SE) } \\
\hline & $\operatorname{SSs}(10)$ & $\operatorname{SSs}(11)$ & $\operatorname{SSd}(10)$ & $\operatorname{SSd}(11)$ & $\operatorname{RSs}(10)$ & $\operatorname{RSs}(11)$ & $\operatorname{RSd}(10)$ & $\operatorname{RSd}(11)$ \\
\hline \multicolumn{9}{|l|}{ Clipping (cm) } \\
\hline$[\mathrm{H}]$ Height & $11.37 \pm 0.26$ & $12.9 \pm 0.54$ & $11.54 \pm 0.34$ & $13.53 \pm 0.5$ & $11.31 \pm 0.34$ & $12.71 \pm 0.49$ & $11.83 \pm 0.37$ & $13.90 \pm 0.56$ \\
\hline [W] Width & $2.82 \pm 0.15$ & $3.52 \pm 0.25$ & $2.92 \pm 0.20$ & $3.50 \pm 0.28$ & $2.32 \pm 0.16$ & $3.18 \pm 0.39$ & $2.79 \pm 0.23$ & $3.49 \pm 0.41$ \\
\hline [B] Base & $0.63 \pm 0.02$ & $0.77 \pm 0.08$ & $0.61 \pm 0.03$ & $0.65 \pm 0.05$ & $0.65 \pm 0.04$ & $0.97 \pm 0.18$ & $0.67 \pm 0.04$ & $0.72 \pm 0.11$ \\
\hline [TBL] Total Branch Length & $54.06 \pm 3.94$ & $68.51 \pm 7.12$ & $49.28 \pm 3.34$ & $70.92 \pm 4.22$ & $49.07 \pm 5.06$ & $68.03 \pm 8.0$ & $47.65 \pm 4.01$ & $69.67 \pm 7.0$ \\
\hline [ML] Mother Length & $8.97 \pm 0.49$ & $10.23 \pm 0.53$ & $8.82 \pm 0.52$ & $10.42 \pm 0.55$ & $9.68 \pm 0.47$ & $9.48 \pm 0.6$ & $10.09 \pm 0.50$ & $11.42 \pm 0.55$ \\
\hline [MW] Mother Width & $0.98 \pm 0.17$ & $1.20 \pm 0.02$ & $0.98 \pm 0.18$ & $1.33 \pm 0.20$ & $0.75 \pm 0.02$ & $1.16 \pm 0.02$ & $0.79 \pm 0.18$ & $1.45 \pm 0.02$ \\
\hline [DL] Daughter Length & $3.93 \pm 0.22$ & $4.57 \pm 0.26$ & $4.14 \pm 0.23$ & $4.85 \pm 0.26$ & $4.18 \pm 0.26$ & $4.69 \pm 0.28$ & $4.18 \pm 0.24$ & $4.60 \pm 0.28$ \\
\hline [DW] Daughter Width & $0.44 \pm 0.04$ & $0.41 \pm 0.03$ & $0.36 \pm 0.03$ & $0.34 \pm 0.02$ & $0.34 \pm 0.02$ & $0.31 \pm 0.02$ & $0.36 \pm 0.02$ & $0.32 \pm 0.02$ \\
\hline$[\mathrm{Bu}] \mathrm{Bud} \#$ & $1.8 \pm 0.34$ & $2.27 \pm 0.66$ & $1.33 \pm 0.42$ & $2.67 \pm 0.59$ & $2.2 \pm 0.47$ & $2.21 \pm 0.52$ & $1.53 \pm 0.36$ & $2.8 \pm 0.73$ \\
\hline [TB\#] Total Branch \# & $11.6 \pm 1.01$ & $15.64 \pm 2.7$ & $9.53 \pm 0.80$ & $12.6 \pm 1.23$ & $11.4 \pm 2.16$ & $13.8 \pm 1.8$ & $9 \pm 0.62$ & $13.2 \pm 1.28$ \\
\hline [DB\#] Total Daughter Branch \# & $9.33 \pm 0.81$ & $11.8 \pm 2.47$ & $7.33 \pm 0.61$ & $10.27 \pm 0.73$ & $9.27 \pm 1.71$ & $10.0 \pm 1.1$ & $7.27 \pm 0.48$ & $10.6 \pm 1.0$ \\
\hline [MB\#] Total Mother Branch \# & $2.27 \pm 0.30$ & $3.00 \pm 0.47$ & $2.2 \pm 0.26$ & $2.73 \pm 0.32$ & $2.13 \pm 0.48$ & $3.8 \pm 1.04$ & $1.73 \pm 0.21$ & $2.6 \pm 0.34$ \\
\hline [c] MB\#:TB\# & $5.91 \pm 0.56$ & $5.78 \pm 5.67$ & $4.92 \pm 0.51$ & $4.86 \pm 0.45$ & $5.8 \pm 0.33$ & $5.01 \pm 0.37$ & $5.78 \pm 0.45$ & $5.83 \pm 0.90$ \\
\hline$[r]$ Ramification rate & \multicolumn{2}{|c|}{$0.200 \pm 0.081$} & \multicolumn{2}{|c|}{$0.146 \pm 0.082$} & \multicolumn{2}{|c|}{$0.142 \pm 0.083$} & \multicolumn{2}{|c|}{$0.146 \pm 0.082$} \\
\hline [PA] Projected Area & $31.90 \pm 1.65$ & $44.19 \pm 4.94$ & $33.49 \pm 2.19$ & $47.72 \pm 4.48$ & $26.26 \pm 1.83$ & $40.77 \pm 5.45$ & $33.12 \pm 3.08$ & $49.82 \pm 6.97$ \\
\hline [PBA] Projected Branch Area & $42.15 \pm 7.20$ & $61.25 \pm 12.53$ & $36.73 \pm 7.42$ & $64.45 \pm 11.13$ & $43.03 \pm 7.99$ & $58.91 \pm 12.81$ & $32.81 \pm 8.2$ & $62.50 \pm 6.41$ \\
\hline$[\mathrm{Po}]$ Porosity \% & $88.90 \pm 0.14$ & $87.81 \pm 0.23$ & $88.90 \pm 0.14$ & $86.66 \pm 0.18$ & $88.90 \pm 0.14$ & $86.62 \pm 0.21$ & $88.90 \pm 0.14$ & $86.41 \pm 0.19$ \\
\hline
\end{tabular}


Table 4.6. Isis hippuris micro-morphological traits of reciprocal transplant and control colony clippings sourced from Ridge 1 and Sampela. Test codes defined in Figure 4.2.

\begin{tabular}{|c|c|c|c|c|c|c|c|c|c|c|}
\hline \multirow{3}{*}{ Phenotypic Trait } & \multicolumn{10}{|c|}{ Dimensions (mean \pm SE) } \\
\hline & \multirow{2}{*}{$\begin{array}{c}\text { Ridge } \\
2010\end{array}$} & \multicolumn{4}{|c|}{2011} & \multirow{2}{*}{$\begin{array}{c}\text { Sampela } \\
2010\end{array}$} & \multicolumn{4}{|c|}{2011} \\
\hline & & RRs & RRd & SRs & SRd & & SSs & SSd & RSs & RSd \\
\hline \multicolumn{11}{|l|}{ Micromorphology (mm) } \\
\hline [PD] Polyp Density $\left(\mathrm{cm}^{-2}\right)$ & $87.13 \pm 2.60$ & $94.63 \pm 3.07$ & $94.43 \pm 6.98$ & $88.56 \pm 5.74$ & $95.33 \pm 6.03$ & $100.21 \pm 5.32$ & $100.77 \pm 4.17$ & $102.74 \pm 6.61$ & $93.91 \pm 4.65$ & $89.15 \pm 7.00$ \\
\hline [Pd] Polyp Depth & $0.081 \pm 0.00$ & $0.074 \pm 0.004$ & $0.063 \pm 0.002$ & $0.081 \pm 0.004$ & $0.064 \pm 0.003$ & $0.081 \pm 0.001$ & $0.077 \pm 0.002$ & $0.066 \pm 0.002$ & $0.068 \pm 0.002$ & $0.060 \pm 0.003$ \\
\hline [pD] Polyp Diameter & $0.035 \pm 0.00$ & $0.031 \pm 0.002$ & $0.023 \pm 0.001$ & $0.033 \pm 0.003$ & $0.018 \pm 0.001$ & $0.030 \pm 0.001$ & $0.030 \pm 0.001$ & $0.021 \pm 0.001$ & $0.033 \pm 0.001$ & $0.029 \pm 0.001$ \\
\hline [ID] Inter-polyp Distance & $0.052 \pm 0.00$ & $0.06 \pm 0.002$ & $0.060 \pm 0.003$ & $0.058 \pm 0.003$ & $0.063 \pm 0.003$ & $0.055 \pm 0.001$ & $0.060 \pm 0.002$ & $0.060 \pm 0.002$ & $0.057 \pm 0.002$ & $0.057 \pm 0.003$ \\
\hline [Cd] Canal Diameter & $0.02 \pm 0.00$ & $0.020 \pm 0.002$ & $0.018 \pm 0.001$ & $0.019 \pm 0.002$ & $0.017 \pm 0.001$ & $0.02 \pm 0.001$ & $0.018 \pm 0.001$ & $0.017 \pm 0.001$ & $0.018 \pm 0.001$ & $0.019 \pm 0.002$ \\
\hline \multicolumn{11}{|l|}{ Sclerites $(\mathrm{mm})$} \\
\hline [CL1] Club Length 1 & $0.073 \pm 0.001$ & $0.072 \pm 0.001$ & $0.074 \pm 0.002$ & $0.074 \pm 0.001$ & $0.073 \pm 0.001$ & $0.068 \pm 0.001$ & $0.069 \pm 0.001$ & $0.069 \pm 0.001$ & $0.071 \pm 0.001$ & $0.069 \pm 0.001$ \\
\hline [CW1] Club mean Width 1 & $0.021 \pm 0.000$ & $0.022 \pm 0.000$ & $0.021 \pm 0.001$ & $0.022 \pm 0.001$ & $0.021 \pm 0.001$ & $0.019 \pm 0.000$ & $0.018 \pm 0.001$ & $0.020 \pm 0.001$ & $0.021 \pm 0.001$ & $0.019 \pm 0.001$ \\
\hline [CL2] Club Length 2 & $0.072 \pm 0.000$ & $0.067 \pm 0.003$ & $0.068 \pm 0.002$ & $0.071 \pm 0.001$ & $0.071 \pm 0.001$ & $0.068 \pm 0.002$ & $0.068 \pm 0.001$ & $0.069 \pm 0.001$ & $0.069 \pm 0.001$ & $0.068 \pm 0.001$ \\
\hline [CW2] Club mean Width 2 & $0.033 \pm 0.000$ & $0.034 \pm 0.001$ & $0.032 \pm 0.001$ & $0.033 \pm 0.001$ & $0.033 \pm 0.001$ & $0.031 \pm 0.001$ & $0.030 \pm 0.001$ & $0.031 \pm 0.001$ & $0.031 \pm 0.001$ & $0.030 \pm 0.001$ \\
\hline [CaL] Capstan Length & $0.115 \pm 0.004$ & $0.117 \pm 0.003$ & $0.116 \pm 0.003$ & $0.1143 \pm 0.003$ & $0.110 \pm 0.002$ & $0.100 \pm 0.002$ & $0.104 \pm 0.003$ & $0.107 \pm 0.002$ & $0.104 \pm 0.002$ & $0.104 \pm 0.002$ \\
\hline [CaW] Capstan mean Width & $0.073 \pm 0.001$ & $0.072 \pm 0.002$ & $0.072 \pm 0.002$ & $0.070 \pm 0.002$ & $0.067 \pm 0.002$ & $0.066 \pm 0.001$ & $0.066 \pm 0.001$ & $0.065 \pm 0.001$ & $0.066 \pm 0.001$ & $0.063 \pm 0.002$ \\
\hline [SL] Spindle Length & $0.165 \pm 0.003$ & $0.176 \pm 0.004$ & $0.170 \pm 0.004$ & $0.174 \pm 0.005$ & $0.165 \pm 0.003$ & $0.161 \pm 0.003$ & $0.169 \pm 0.004$ & $0.168 \pm 0.004$ & $0.171 \pm 0.005$ & $0.163 \pm 0.005$ \\
\hline [SW] Spindle Width & $0.067 \pm 0.001$ & $0.075 \pm 0.002$ & $0.073 \pm 0.002$ & $0.071 \pm 0.002$ & $0.069 \pm 0.002$ & $0.063 \pm 0.001$ & $0.063 \pm 0.001$ & $0.062 \pm 0.001$ & $0.069 \pm 0.002$ & $0.065 \pm 0.002$ \\
\hline
\end{tabular}


Table 4.7. Isis hippuris optical parameters of reciprocal transplant and control colony clippings sourced from Ridge 1 and Sampela.

Test codes defined in Figure 4.2.

\begin{tabular}{|c|c|c|c|c|c|}
\hline \multirow{3}{*}{ Phenotypic Trait } & \multicolumn{5}{|c|}{ Dimensions $($ mean $\pm \mathrm{SE})$} \\
\hline & \multirow{2}{*}{ Ridge 2010} & \multicolumn{4}{|c|}{2011} \\
\hline & & RRs & RRd & SRs & SRd \\
\hline \multicolumn{6}{|l|}{ Optical Parameters } \\
\hline [ZD] Zooxanthellar Density $\left(\right.$ cells $\left.\mathrm{cm}^{-2}\right) \times 10^{6}$ & $6.71 \pm 0.33$ & $5.07 \pm 0.20$ & $3.39 \pm 0.94$ & $4.98 \pm 0.58$ & $3.22 \pm 0.19$ \\
\hline [MI] Mitotic Index \% & $1.93 \pm 0.16$ & $1.36 \pm 0.17$ & $1.92 \pm 0.91$ & $2.81 \pm 0.51$ & $2.48 \pm 0.32$ \\
\hline [Zd] Zooxanthellar mean Diameter $(\mu \mathrm{m})$ & $7.449 \pm 0.027$ & $7.767 \pm 0.026$ & $7.834 \pm 0.027$ & $8.164 \pm 0.027$ & $7.859 \pm 0.029$ \\
\hline [SA:V] Zooxanthellar SA:V & $0.813 \pm 0.003$ & $0.779 \pm 0.003$ & $0.775 \pm 0.003$ & $0.741 \pm 0.003$ & $0.771 \pm 0.003$ \\
\hline$\left[a^{*}\right]$ Chl $a$ Specific Absorption $\left(\mathrm{m}^{-2} \mathrm{mg} \operatorname{chl} a\right)$ & $0.030 \pm 0.005$ & $0.033 \pm 0.007$ & $0.028 \pm 0.001$ & $0.046 \pm 0.005$ & $0.040 \pm 0.004$ \\
\hline$[A] \mathrm{Chl} a$ Absorbance $\left(\mu \mathrm{g} \mathrm{cm}^{-2}\right)$ & $13.72 \pm 1.01$ & $12.28 \pm 1.88$ & $14.25 \pm 3.52$ & $6.34 \pm 0.85$ & $6.67 \pm 0.61$ \\
\hline [CZ] Chl $a$ Absorbance $\left(\mathrm{pg} \mathrm{cell}^{-1}\right)$ & $2.19 \pm 0.17$ & $2.38 \pm 0.35$ & $4.06 \pm 0.27$ & $1.58 \pm 0.31$ & $2.21 \pm 0.25$ \\
\hline$\left[\Delta \mathrm{F} / \mathrm{Fm}^{\prime}\right]$ Light Adapted Yield & $0.520 \pm 0.013$ & $0.564 \pm 0.006$ & $0.550 \pm 0.006$ & $0.579 \pm 0.007$ & $0.587 \pm 0.006$ \\
\hline
\end{tabular}

\begin{tabular}{|c|c|c|c|c|c|}
\hline \multirow{3}{*}{ Phenotypic Trait } & \multicolumn{5}{|c|}{ Dimensions $($ mean $\pm \mathrm{SE})$} \\
\hline & \multirow{2}{*}{ Sampela 2010} & \multicolumn{4}{|c|}{2011} \\
\hline & & SSs & SSd & RSs & RSd \\
\hline \multicolumn{6}{|l|}{ Optical Parameters } \\
\hline [ZD] Zooxanthellar Density $\left(\right.$ cells $\left.\mathrm{cm}^{-2}\right) \times 10^{6}$ & $5.47 \pm 0.20$ & $5.06 \pm 0.52$ & $3.05 \pm 0.20$ & $5.00 \pm 0.43$ & $3.27 \pm 0.31$ \\
\hline [MI] Mitotic Index \% & $2.57 \pm 0.17$ & $2.38 \pm 0.24$ & $1.82 \pm 0.29$ & $1.77 \pm 0.27$ & $1.90 \pm 0.30$ \\
\hline [Zd] Zooxanthellar mean Diameter $(\mu \mathrm{m})$ & $7.912 \pm 0.028$ & $8.213 \pm 0.027$ & $8.184 \pm 0.027$ & $8.303 \pm 0.025$ & $8.127 \pm 0.027$ \\
\hline [SA:V] Zooxanthellar SA:V & $0.764 \pm 0.002$ & $0.737 \pm 0.002$ & $0.739 \pm 0.002$ & $0.728 \pm 0.002$ & $0.744 \pm 0.002$ \\
\hline$\left[a^{*}\right]$ Chl $a$ Specific Absorption $\left(\mathrm{m}^{-2} \mathrm{mg} \operatorname{chl} a\right)$ & $0.037 \pm 0.003$ & $0.026 \pm 0.003$ & $0.026 \pm 0.003$ & $0.020 \pm 0.002$ & $0.027 \pm 0.009$ \\
\hline$[A] \mathrm{Chl} a$ Absorbance $\left(\mu \mathrm{g} \mathrm{cm}^{-2}\right)$ & $10.93 \pm 0.87$ & $11.15 \pm 0.51$ & $10.41 \pm 0.42$ & $14.38 \pm 1.83$ & $10.21 \pm 0.85$ \\
\hline [CZ] Chl $a$ Absorbance (pg cell ${ }^{-1}$ ) & $2.04 \pm 0.15$ & $2.37 \pm 0.27$ & $3.50 \pm 0.27$ & $3.12 \pm 0.44$ & $3.37 \pm 0.31$ \\
\hline [ $\Delta \mathrm{F} / \mathrm{Fm}$ '] Light Adapted Yield & $0.530 \pm 0.012$ & $0.537 \pm 0.007$ & $0.527 \pm 0.006$ & $0.589 \pm 0.005$ & $0.580 \pm 0.006$ \\
\hline
\end{tabular}



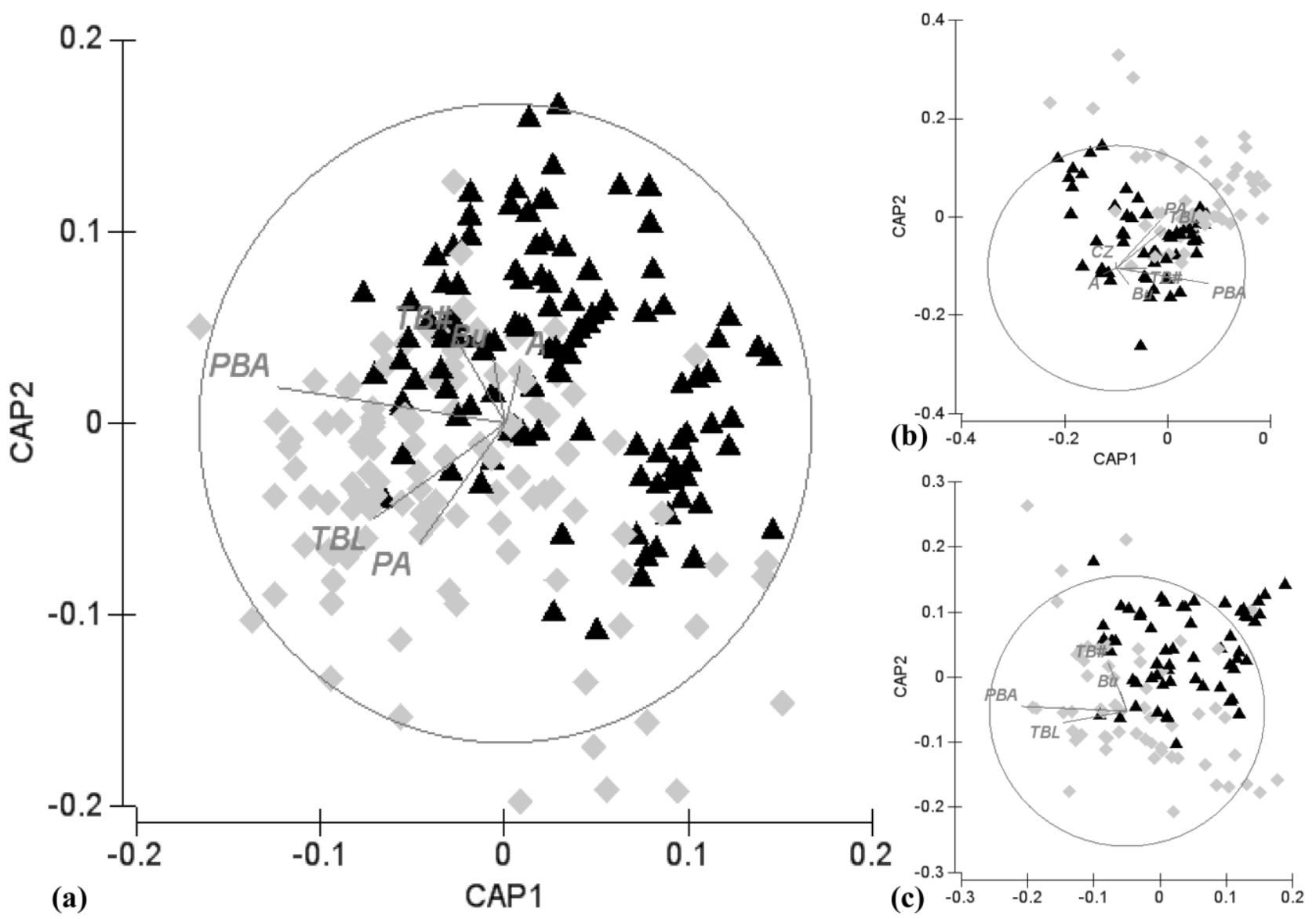

Figure 4.6. CAP ordinations of (a) all Isis hippuris phenotypic traits and tests, with the same model repeated for (b) the Ridge and (c) Sampela. Test labels $2010(\boldsymbol{A})$ and $2011(\diamond)$; see table 4.4 - 7 for vector trait codes.

Results from the full PERMANOVA model across all tests revealed annual, site and depth differences with no interaction effects (Table 4.8; Figure 4.6). Principle traits overlaying such differences were at the colony (PBA 13\%, Bu 9\%, PA, TBL \& TB\# 6\% dissimilarity) and chl $a$ absorbance levels (A 6\% dissimilarity). Models segregated by site show that differences in depth and chl $a$ irradiance response $[A]$ from the full model, were specifically attributed to the Ridge (Table 4.8; Figure 4.6a,b). Ridge dissimilarities between depth and site averaged $11 \%$ for PBA and $7 \%$ for $\mathrm{Bu}, \mathrm{PA}$ and $A$, whereas only the annual difference was significant at Sampela primarily being at the colony branching level (PBA 14\%, Bu 9\%, TB\# 6\%, TBL 5\% dissimilarity). These results imply that growth and chl $a$ absorbance efficiency maintain fixed and plastic responses of the holobiont phenotype for Sampela and Ridge source colonies respectively. To further investigate this, the same statistical model was parsed into each phenotypic module (colony, polyp, sclerite and optical dynamics) testing for both within and between factor variance (Table 4.8). 
Table 4.8. PERMANOVA of Isis hippuris test colony phenotypic traits. Repetitive analyses results on full (all tests and traits) and parsed models between sites (Ridge, Sampela) and phenotypic modules (colony, polyps, sclerites and optical parameters). Note, only interactions with a significant effect are presented. $* P<0.01$, **P $<0.001$, ***P $<0.0001$, ns, not significant. Y, S and D, denotes year, site and depth respectively.

\begin{tabular}{|c|c|c|c|c|c|c|c|c|c|}
\hline \multirow{2}{*}{ Source } & \multirow[b]{2}{*}{$d f$} & \multicolumn{2}{|c|}{ All Tests } & \multicolumn{3}{|c|}{ Ridge } & \multicolumn{3}{|c|}{ Sampela } \\
\hline & & SS & pseudo- $F$ & $d f$ & SS & pseudo- $F$ & $d f$ & SS & pseudo- $F$ \\
\hline \multicolumn{10}{|c|}{ All Variables } \\
\hline Year & 1 & 1410.2 & $33.292^{* * *}$ & 1 & 799.05 & $22.427^{* * *}$ & 1 & 720.5 & $14.659^{* * *}$ \\
\hline Site & 1 & 471.27 & $11.126^{* * * *}$ & 1 & 76.658 & $2.1515^{\mathrm{ns}}$ & 1 & 54.257 & $1.1039^{\text {ns }}$ \\
\hline Depth & 1 & 1115.66 & $2.7304^{*}$ & 1 & 1117.25 & $3.2908^{*}$ & 1 & 74.286 & $1.5114^{\mathrm{ns}}$ \\
\hline Total & 225 & 11522 & & 107 & 4711.1 & & 117 & 6349.5 & \\
\hline \multicolumn{10}{|c|}{ Colony } \\
\hline Year & 1 & 1962.5 & $32.364^{* * *}$ & 1 & 1066.8 & $22.868^{* * *}$ & 1 & 934.14 & $12.391^{* *}$ \\
\hline Site & 1 & 706.51 & $11.651^{* *}$ & 1 & 43.754 & $0.93791^{\mathrm{ns}}$ & 1 & 84.12 & $1.1158^{\mathrm{ns}}$ \\
\hline Depth & 1 & 104.27 & $1.7195^{\mathrm{ns}}$ & 1 & 131.4 & $2.8166^{*}$ & 1 & 76.697 & $1.0173^{\mathrm{ns}}$ \\
\hline Total & 225 & & & 107 & 6045.6 & & 117 & 9462.8 & \\
\hline \multicolumn{10}{|c|}{ Polyps } \\
\hline Year & 1 & 26.016 & $4.3663^{*}$ & 1 & 21.475 & $4.0458^{*}$ & 1 & 14.972 & $2.2393^{\mathrm{ns}}$ \\
\hline Site & 1 & 18.603 & $3.1222^{\mathrm{ns}}$ & 1 & 0.5288 & $0.09625^{\mathrm{ns}}$ & 1 & 13.532 & $2.0238^{\text {ns }}$ \\
\hline Depth & 1 & 7.501 & $1.2589^{\mathrm{ns}}$ & 1 & 4.1891 & $0.78922^{\mathrm{ns}}$ & 1 & 5.17 & $0.77325^{\mathrm{ns}}$ \\
\hline Total & 225 & 1371.9 & & 107 & 564.74 & & 117 & 788.14 & \\
\hline \multicolumn{10}{|c|}{ Sclerites } \\
\hline Year & 1 & 110.62 & $8.4485^{* * *}$ & 1 & 70.468 & $5.0004^{*}$ & 1 & 47.065 & $3.7412^{*}$ \\
\hline Site & 1 & 443.85 & $33.899^{* * *}$ & 1 & 15.792 & $1.1206^{\mathrm{ns}}$ & 1 & 11.916 & $11.916^{\mathrm{ns}}$ \\
\hline Depth & 1 & 26.615 & $2.0327^{\mathrm{ns}}$ & 1 & 14.884 & $1.0562^{\mathrm{ns}}$ & 1 & 14.029 & $14.029^{\text {ns }}$ \\
\hline Total & 225 & 3456.9 & & 107 & 1544.3 & & 117 & 1474.4 & \\
\hline \multicolumn{10}{|c|}{ Optical Parameters } \\
\hline Year & 1 & 1190.7 & $29.61^{* * *}$ & 1 & 790.94 & $18.661^{* * *}$ & 1 & 785.07 & $23.491^{* * * *}$ \\
\hline Site & 1 & 203.81 & $5.0682^{*}$ & 1 & 365.71 & $8.6282^{* *}$ & 1 & 40.362 & $1.2077^{\mathrm{ns}}$ \\
\hline Depth & 1 & 249.62 & $6.2075^{*}$ & 1 & 200.95 & $4.7411^{*}$ & 1 & 121.26 & $3.6284^{\mathrm{ns}}$ \\
\hline $\mathbf{Y x S}$ & 1 & 383.72 & $9.5422^{* *}$ & 1 & 335.96 & $7.9264^{* *}$ & 1 & 53.075 & $1.5881^{\mathrm{ns}}$ \\
\hline YxD & 1 & 324.82 & $8.0775^{* *}$ & 1 & 241.63 & $5.7008^{*}$ & 1 & 143.49 & $4.2936^{*}$ \\
\hline SxD & 1 & 53.949 & $1.3416^{\mathrm{ns}}$ & 1 & 30.831 & $0.7274^{\mathrm{ns}}$ & 1 & 34.472 & $1.0315^{\mathrm{ns}}$ \\
\hline YxSxD & 1 & 36.672 & $0.91195^{\mathrm{ns}}$ & 1 & -11.222 & - & 1 & 2.4987 & $0.074766^{\mathrm{ns}}$ \\
\hline Total & 225 & 11201 & & 107 & 6150.8 & & 117 & 4856.7 & \\
\hline \multicolumn{10}{|c|}{ Optical Parameters: 2-Factor Model } \\
\hline Site & 1 & 203.81 & $4.2269^{* *}$ & 1 & 365.71 & $6.8263^{* *}$ & 1 & 40.362 & $0.98718^{\mathrm{ns}}$ \\
\hline Depth & 1 & 249.62 & $5.1772^{*}$ & 1 & 200.95 & $3.751^{*}$ & 1 & 121.26 & $2.9658^{*}$ \\
\hline Total & 225 & 11201 & & 107 & 6150.8 & & 117 & 4856.7 & \\
\hline
\end{tabular}



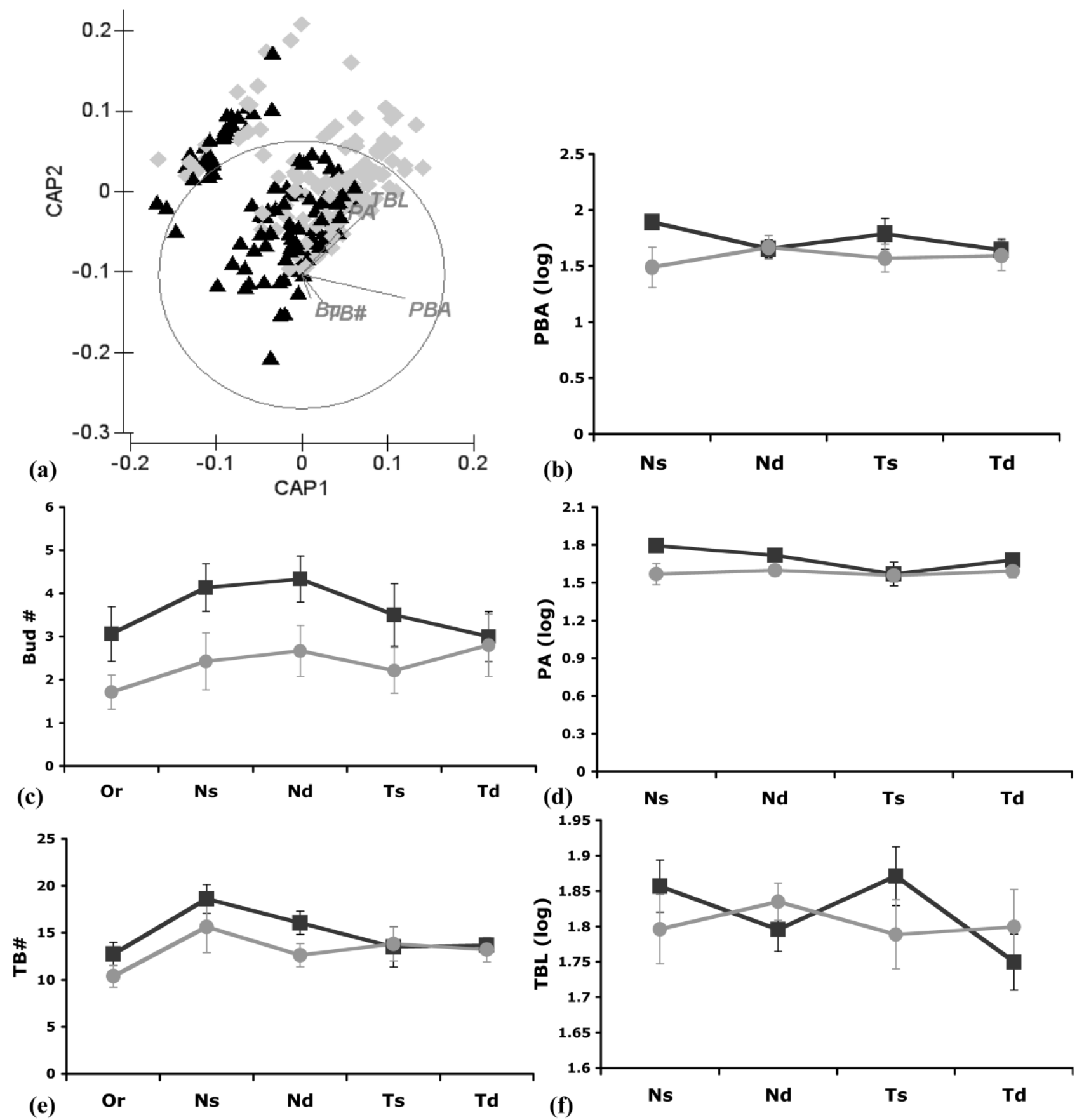

Figure 4.7. Isis hippuris morphological traits with: (a) CAP ordination of all test traits. Reaction norms of annual growth (adjusted for positive association, see equation 1) for branching parameters between 2010 - 2011 with (b) the projected branch area [PBA], (d) the projected colony area [PA], (f) the total branch length [TBL]. Reaction norms of (c) bud number and (e) total branch number [TB\#]. Factor level labels $2010(\boldsymbol{\Delta})$ and $2011(\diamond)$, Ridge $(-\boldsymbol{-})$ and Sampela $\left(\boldsymbol{-}^{-}\right)$, and Or, origin; Ns, native shallow; $\mathrm{Nd}$, native deep; Ts, transplant shallow; Td, transplant deep. 


\section{Colony Morphometrics}

Colony growth was one of the principal drivers of annual site differences (see Table $4.4-5 \&$ 4.8), typically greater for Ridge colonies with a notable reduction at depth (Figure 4.7b, f). Correction for positive association through annual growth (Eq.1; Figure 4.7) reveals both growth rate and colony form was relatively preserved in Sampela colonies compared to those from the Ridge. Initial bursts in branching and bud number are likely attributed to increased resource allocation to colony growth and form (Figure $4.7 \mathrm{c}$, e). Reaction norms reveal $V_{G}$ and $V_{E}$ variance, particularly for budding and less so for total branch number. Total branch length accounted for the variability in projected branch area, mirroring the pattern of greater to reduced branching intensity for Ridge source colonies with increased depth (as a function of light intensity). To a far lesser extent was the opposite true for Sampela, increasing branch length with increased light attenuation, highlighting a clear $V_{G x E}$ interaction between the two morphotypes (Figure 4.7b, f). Projected area (PA) remained constant for Sampela yet varied slightly for the Ridge, likely a reflection of differential growth; finally, $V_{G}$ existed between both source colonies with environmentally induced plasticity at Sampela shallow for Ridge colonies (Figure 4.7d). Trait dissimilarities were similar however for both sites (PBA 15\% and 18\%, Bu $11 \%$ and $12 \%$ for Ridge [also with PA 9\%] and Sampela with TB\# \& TBL at 7\%) often juxtaposing each other as with the case of PBA and TBL $\left(V_{G x E}\right)$. Branching trait integration probably explains such differences, particularly facilitating Ridge colony plasticity relative to increased water flow and differential light availability. Branching trait integration was also evident in the parameter $c$, which remained constant, with no evidence for dissimilarity between tests in alignment with previous studies (Table 4.4 - 5; Sánchez 2004). Branch width was not as independently influential as branch length and number, but was reflected in PBA. Taken together, within phenotypic module integration between branching traits was evident in the differential morphotype responses to environmental change. 

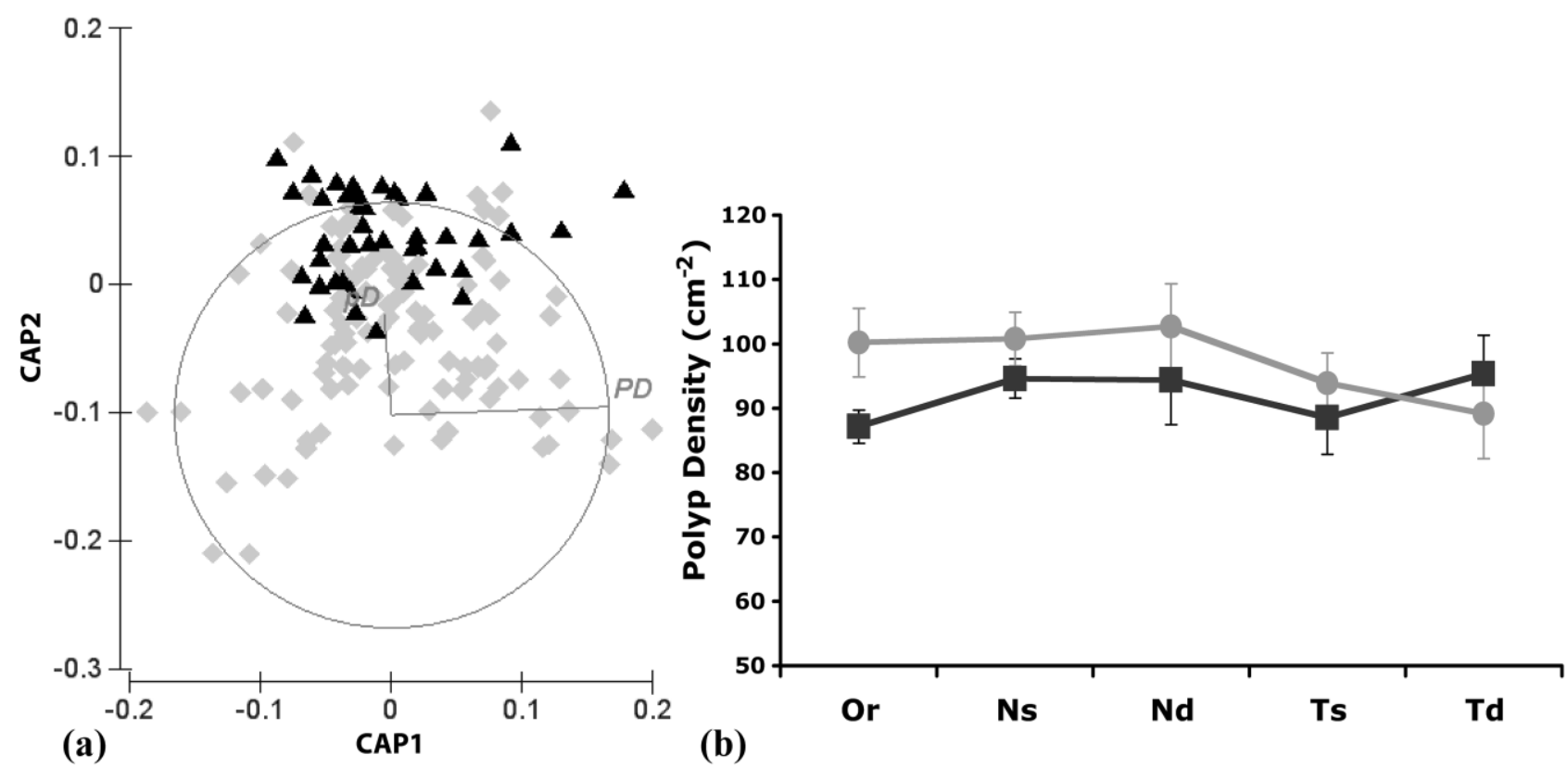

Figure 4.8. (a) CAP ordination of Isis hippuris polyp dimensions with (b) reaction norms of polyp density as the most variable trait observed. Factor level labels $2010(\boldsymbol{A})$ and $2011(\diamond)$, Ridge $(\boldsymbol{-})$ and Sampela (-), and Or, origin; Ns, native shallow; Nd, native deep; Ts, transplant shallow; Td, transplant deep.

\section{Polyps}

Little variation in polyp dimensions was observed with only an annual significant difference at the Ridge (Table 4.8). On average there was $85 \%$ dissimilarity due to polyp densities in Ridge colonies irrespective of a clear reduction in polyp density in Sampela transplants at the Ridge (Figure 4.8b; Table 4.8). Thus, there appears to be a similar response to depth by both source colonies at the opposite environment suggesting additional nutritional factors are of influence as well as a $V_{E x G}$ interaction. Furthermore, both polyp density and diameter reduced at depth specifically at the Ridge, but with only minimal influence on the analyses for the latter $(\mathrm{pD}$; Table 4.6). Polyp depth was considerably reduced in nearly all but one of the transplant blocks (Figure 4.5). This block was omitted from the remainder of the analyses due to disturbance (see above), however it is noteworthy that two of the three remaining clippings were externally brooding pink eggs also observed in the surrounding native reef colonies (Figure 4.5), but not present on any other reef within the area, only Sampela. Dissection also revealed the presence of eggs within polyps of 2 control colonies (see SSs polyp depth; Table 4.6) in 2011, yet eggs were regularly encountered within source colony polyps in 2010 with no site preference suggesting resource allocation to growth not reproduction in 2011. 

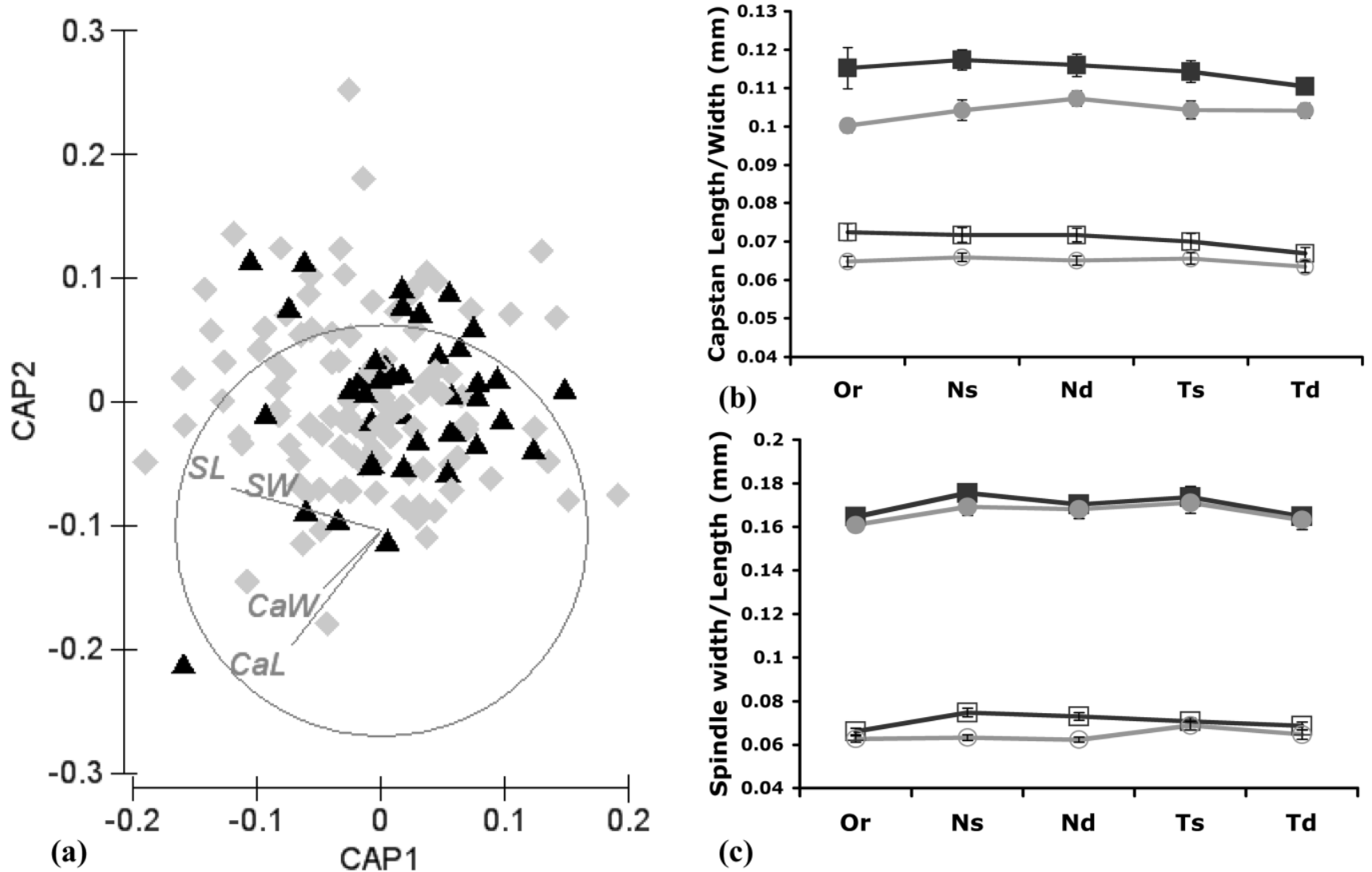

Figure 4.9. Isis hippuris annual sclerite size distribution across all tests. (a) CAP analyses of sclerite variation with promine spind $\quad \begin{array}{ll}\mathbf{R}[\mathbf{S L}] \\ \mathbf{S}[\mathbf{S L}]\end{array}$ ns. Sclerite (sı $\mathbf{R}[\mathbf{S L}]$ epresented as reaction norms for (b) capstan, and (c)

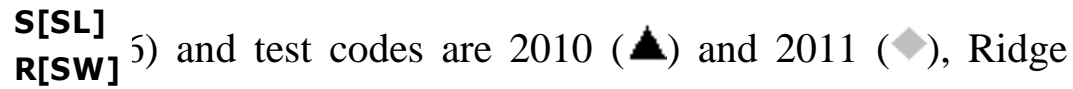

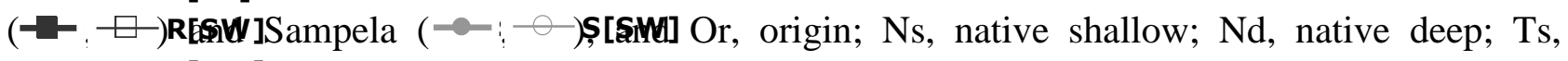
transplant s[SWllow; Td, transplant deep.

\section{Sclerites}

Ns Td Ndiations $\mathbf{T} \mathbf{\$}$ scleriteddimensions were primarily in coenenchyme surface capstans and subsurface spindles, with dissimilarities of SL 24 and 29\%, CaL 19.5\% and 17\%, SW 13\% and 12\%, CaW 13 and 10\% for Ridge and Sampela respectively (Figure 4.9). A consistent decrease in sclerite size with reduced irradiance was observed for all tests, most markedly with spindle length and within colonies particularly from the Ridge (Figure 4.9b, c). Most notable, however, was the consistency in reduced size in all sclerites from Sampela suggesting $V_{G}$ and $V_{E}$ in both cases. 

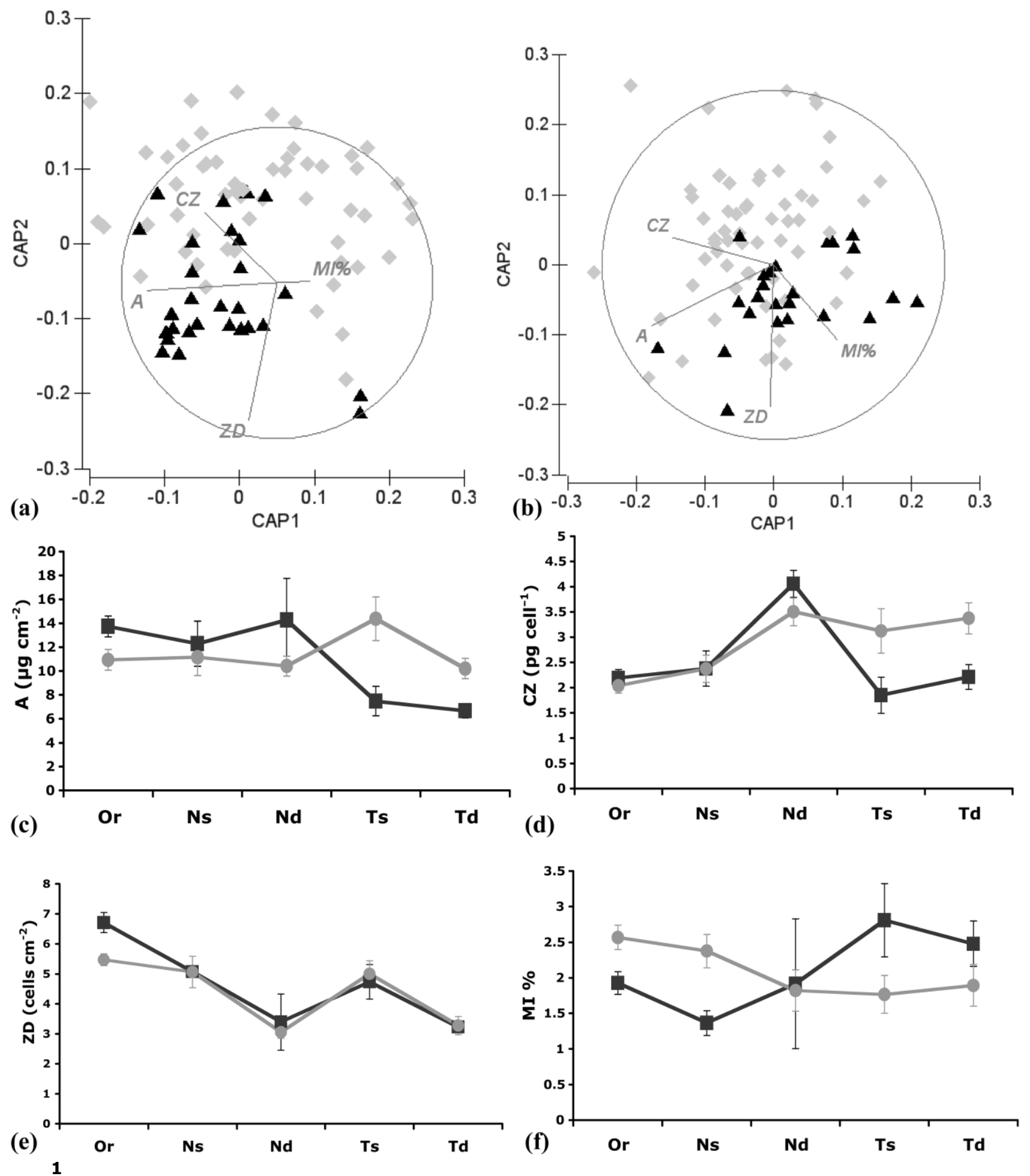

Figuffe 4.10. Optical parameters from test colonies with CAP constrained ordinations of (a) Ridge

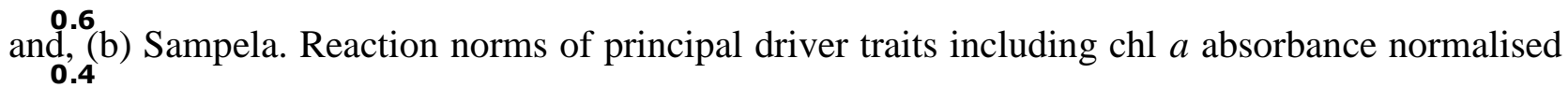
to fe.2 surface area, (d) per zooxanthellar cell, (e) zooxanthellar dencitv and (f) mitotic index. Test code are: deep $(\boldsymbol{\Delta})$ and shallow $(\diamond)$, Ridge $(-\mathbf{R}$ and Sampela $(-\bullet \mathbf{S}$, and Or, origin; Ns, native

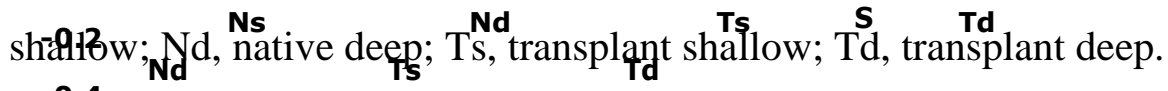

$-0.4$

Oø!dical Parameters

FA 8 influence of light-dependent phenotypic responses was most apparent on Ridge source colonies (Table 4.8; Figure 4.10). Significant main effects of both full and site-specific optical 
trait PERMANOVA models were countered by interactions between year $\mathrm{x}$ depth for both sites in addition to year $\mathrm{x}$ site for the Ridge (Table 4.8). This suggests that the differences between source colony responses to environmental change were variable; neither adjusting to the same magnitude nor in the same way when placed in the opposite environment (Figure 4.10). Pairwise differences were significant between sites in both years $(P \leq 0.01)$, depth in $2011(P<0.0001)$, and depth within sites $(P \leq 0.04)$. Naturally, pairwise comparisons revealed no difference between depths in 2010 considering all source colonies were selected at shallow optical equivalent depths (Figure 4.3d). Therefore, a two-factor model was constructed, omitting the factor 'year'. This confirmed pairwise comparisons for depth at both sites on transplantation, but no difference within Sampela colonies suggested a consistent response to reduced irradiance by depth irrespective of location or colony source (Table 4.8). Average dissimilarities across all tests in alignment with CAP vector overlays were $A 30 \%$ and $27 \%, C Z 24 \%$ and $24.5 \%$, MI $18 \%$ and $21 \%$, and ZD $22 \%$ and $21 \%$ for the Ridge and Sampela respectively. Chl $a$ concentration $[A]$ increased with irradiance being consistently lower in Sampela colonies until transplanted to the shallow Ridge and vice versa, with a stronger response from Ridge colonies to Sampela (Figure 4.10c). This would suggest a $V_{G x E}$ interaction response between I. hippuris holobionts across the two sites. Chl $a$ per cell $[C Z]$ increased with depth in both cases but also dropped considerably in Ridge transplants to Sampela and increased from Sampela to Ridge at both depths (Figure 4.10d).

Overall, reaction norms demonstrate magnitudinal differences indicating $V_{G}$ and $V_{E}$ responses between holobionts to environmental change. Zooxanthellae cell density [ZD] drove differences between depth but not site, both morphotypes responding similarly to their environments indicating a $V_{E}$ and $V_{G x E}$ interaction, i.e., plastic response (Figure 4.10e). Cell division was greater at Sampela irrespective of colony origin, being most dramatic in Ridge colonies (Figure 4.10f). Furthermore, zooxanthellar cell size was slightly larger at Sampela irrespective of treatment: average $8.2 \mu \mathrm{m}$ and $7.8 \mu \mathrm{m}$ for Sampela and Ridge respectively. Zooxanthellae SA:V was inversely related to cell density at the Ridge providing greater diffusion efficiency. Coupled with pigment concentration and density, this provides no evidence for self-shading through packing in test colonies from the Ridge at depth. Zooxanthellae SA:V in Sampelan colonies however, were relatively unresponsive to environmental change with consistently larger cells (Table 4.7). Chl $a$ specific absorption [ $\left.a^{*}\right]$ appeared to have little effect on Sampela 


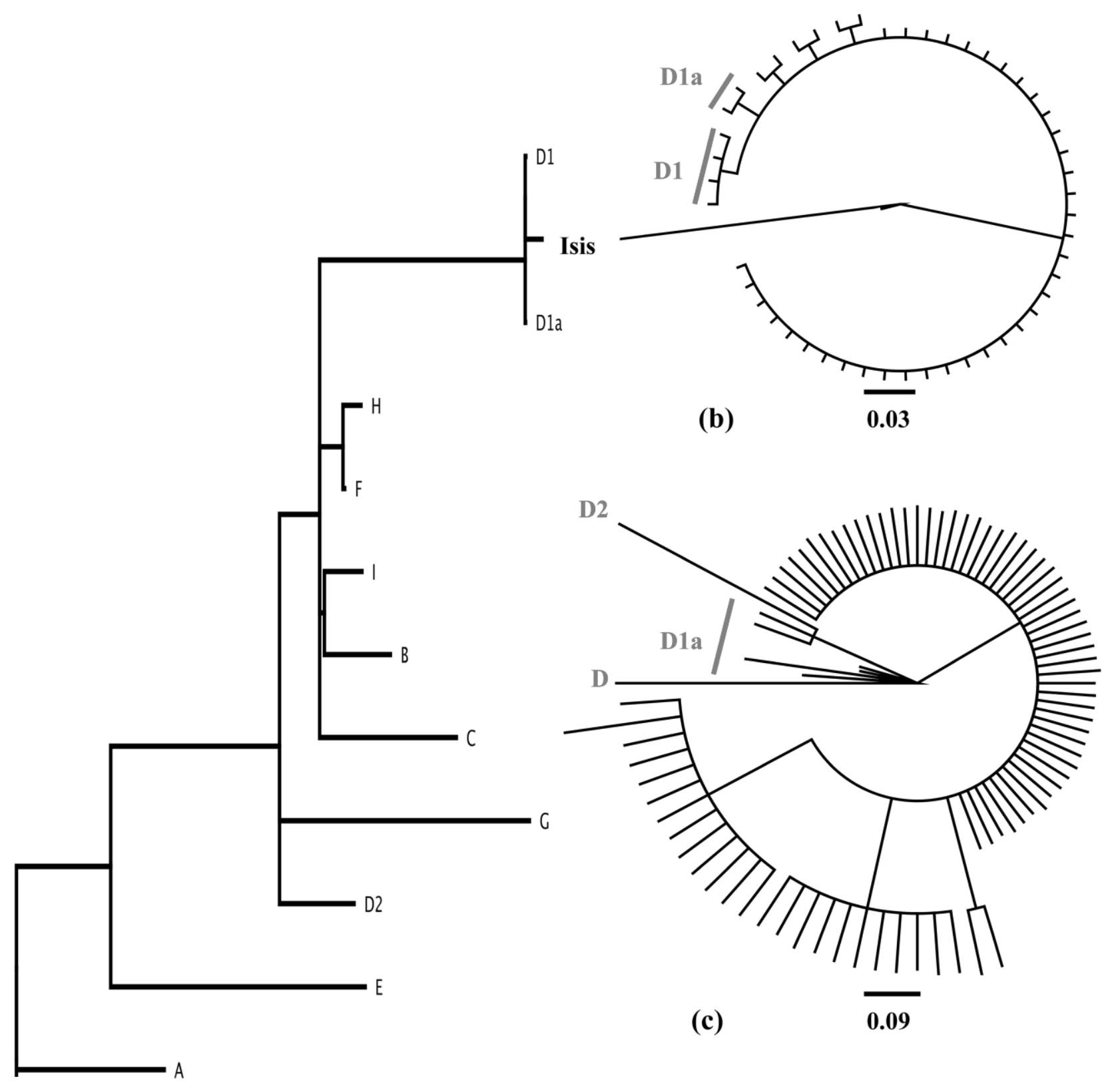

(a)

Csimplex

Figure 4.11. Phylograms of Symbiodinium clades based on neighbour joining (NJ), bayesian inference (BI), and maximum likelihood (ML) analyses (1000 bootstrap) of the (a) mitochondrialencoded cytochrome oxidase COX1 region (Takabayashi et al. 2004), unrooted trees (NJ/ML) of (b) the plastid-coding $p s b A$ minicircle, and (c) the internal transcribed spacer (ITS) region. Red bars and letters indicate clade D GenBank sequences. Scale bars correspond to base changes per site. 
colonies decreasing with depth at the Ridge, the latter also the case with Ridge colonies plus a consistent increase on transplantation to Sampela for both depths (Table 4.7). Effective quantum yield $[\Delta \mathrm{F} / \mathrm{Fm}$ '] in light-adapted (in situ) colonies revealed an increase in those transplanted to and from Sampela with only a slight decrease at the native depth (Table 4.7).

Taken together, in virtually all optical traits the response to transplantation was greater and/or more variable in colonies from the Ridge, further corroborating an increased plastic response of the Ridge holobiont to environmental change compared to a somewhat more restrained and often contrasting response within Sampelan colonies.

\section{Environmental Parameters}

With no annual differences between measured abiotic traits all DISTLMforward analyses were performed on within (depth) and between site differences based on reduced test models as outlined in Table 4.8. Results comparing all phenotypic traits within and between sites showed that light $\left(\mathrm{K}_{\mathrm{d}(\mathrm{PAR})}\right)$, water flow and turbidity explained $69.2 \%$ of test colony differences. Segregation by source colony site revealed that water flow, light $\left(\mathrm{K}_{\mathrm{d}(\mathrm{PAR})}\right)$ and turbidity explained $57.5 \%$ for Ridge colony differences and that sediment, light $\left(\mathrm{K}_{\mathrm{d}(\mathrm{PAR})}\right)$ and water flow explained $69.5 \%$ for Sampela source colonies. These patterns were consistent for all phenotypic module and integrated trait analyses.

\section{Symbiodinium Genetic Analyses}

Symbiodinium were identified using the mitochondrial marker COX1, rDNA ITS2, and the plastid-coding $p s b A$ minicircle. A total of 186 COX1 sequences were recovered for phylogenetic analyses with previously published results (Table 4.2; Figure 4.11). Without exception all sequences were a novel strain of D1a, differing by only a single base pair from previously published results (Takishita et al. 2004; Figure 4.11). Of the 80 ITS2 cloned sequences recovered, there included no less than 7 for each test, a total of 9 haplotypes with $1-6$ bp difference between them and 1-11 bp difference from previous studies (Table 4.2), and thus also novel. Sequenced clones from the $p s b A$ marker totaled 46 with 4 or more clones for each test and 5 haplotypes 1 - 2 bp apart with 1 - 3 bp difference from previous work (D1a, Takishita et al. 2003). No patterns of annual, site or depth specificity were present between haplotypes for either marker. Of the three gene regions and methods utilized in this study, there was no evidence of symbiont shuffling within the I. hippuris holobiont across all tests. 
Table 4.9. Phenotypic trait correlation table with Ridge above the diagonal and Sampela below. Values indicate: $\geq 0.7$ very strong positive correlation, 0.4 to 0.69 strong positive correlation, 0.20 to 0.39 weak to moderate positive correlation, -0.19 to 0.19 negligible, -0.20 to -0.39 weak to moderate negative correlation, -0.4 to -0.69 strong negative correlation, $\geq-0.7$ very strong negative correlation. Trait codes see Tables 4.5-7.

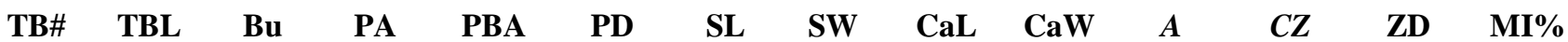

\begin{tabular}{|c|c|c|c|c|c|c|c|c|c|c|c|c|c|c|}
\hline TB\# & 1 & 0.739 & 0.395 & 0.576 & 0.735 & -0.140 & 0.062 & 0.126 & -0.004 & -0.013 & -0.280 & -0.192 & -0.054 & 0.017 \\
\hline TBL & 0.721 & 1 & 0.285 & 0.693 & 0.865 & 0.004 & 0.350 & 0.395 & 0.119 & 0.057 & -0.224 & -0.113 & -0.103 & -0.055 \\
\hline Bu & 0.339 & 0.279 & 1 & 0.319 & 0.309 & -0.102 & 0.156 & 0.116 & 0.002 & -0.067 & -0.232 & -0.166 & -0.045 & 0.019 \\
\hline PA & 0.476 & 0.733 & 0.222 & 1 & 0.632 & -0.100 & 0.238 & 0.401 & 0.080 & -0.123 & -0.281 & -0.054 & -0.274 & -0.099 \\
\hline PBA & 0.756 & 0.904 & 0.256 & 0.607 & 1 & -0.031 & 0.112 & 0.248 & -0.002 & 0.004 & -0.245 & -0.167 & -0.059 & 0.024 \\
\hline PD & -0.166 & -0.103 & -0.029 & -0.166 & -0.095 & 1 & 0.226 & 0.089 & 0.065 & 0.007 & -0.048 & -0.061 & -0.003 & 0.017 \\
\hline SL & 0.303 & 0.434 & 0.196 & 0.264 & 0.349 & -0.203 & 1 & 0.689 & 0.440 & 0.198 & -0.081 & -0.075 & -0.002 & -0.061 \\
\hline SW & 0.313 & 0.387 & 0.119 & 0.265 & 0.330 & -0.067 & 0.703 & 1 & 0.350 & 0.332 & -0.020 & 0.065 & -0.128 & -0.208 \\
\hline CaL & 0.071 & 0.077 & 0.230 & 0.127 & 0.009 & -0.066 & 0.154 & 0.155 & 1 & 0.482 & 0.145 & 0.030 & 0.124 & -0.225 \\
\hline CaW & -0.021 & 0.027 & 0.126 & 0.083 & -0.039 & -0.003 & 0.331 & 0.345 & 0.559 & 1 & 0.142 & 0.075 & 0.062 & 0.090 \\
\hline $\boldsymbol{A}$ & 0.148 & 0.209 & 0.006 & -0.011 & 0.212 & 0.275 & -0.060 & 0.172 & -0.218 & -0.078 & 1 & 0.751 & 0.207 & -0.110 \\
\hline$C Z$ & 0.171 & 0.318 & 0.047 & 0.164 & 0.286 & 0.224 & -0.040 & 0.095 & -0.098 & -0.087 & 0.714 & 1 & -0.487 & -0.067 \\
\hline ZD & -0.073 & -0.209 & -0.073 & -0.262 & -0.158 & 0.036 & -0.024 & 0.072 & -0.127 & 0.027 & 0.192 & -0.548 & 1 & -0.061 \\
\hline MI\% & -0.120 & -0.230 & -0.032 & -0.156 & -0.203 & -0.036 & -0.007 & -0.087 & -0.092 & -0.096 & -0.124 & -0.280 & 0.244 & 1 \\
\hline
\end{tabular}

\section{Multivariate Analyses of Integration}

Multivariate analyses revealed 14 prominent phenotypic traits within and between I. hippuris morphotypes that drive the differences between tests; PBA, Bu, PA, TBL, TB\#, PD, CaL, CaW, SL, SW, A, ZD, CZ, and MI. These traits were isolated and re-modeled confirming their distributional influence (year: pseudo- $F$ 24.111/16.21, $P<0.0001$ Ridge and Sampela; with additional site; pseudo- $F$ 3.001, $P<0.03$ and depth: pseudo- $F 3.7882, P<0.01$ for the Ridge), as were the remaining traits as a likely indication of canalised integration confirming magnitudinal trait differences within and between the two morphotypes (site: pseudo- $F$ 13.084, 


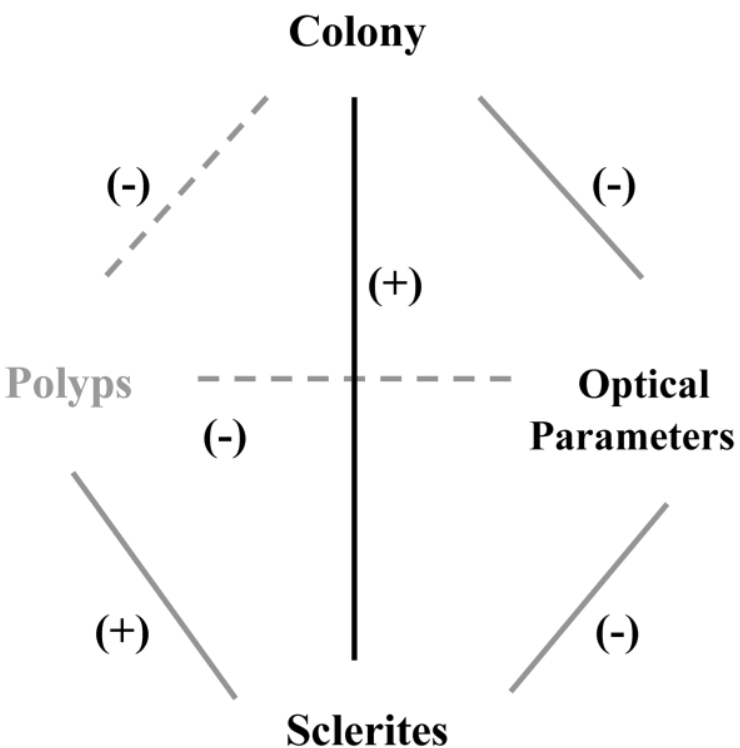

(a)

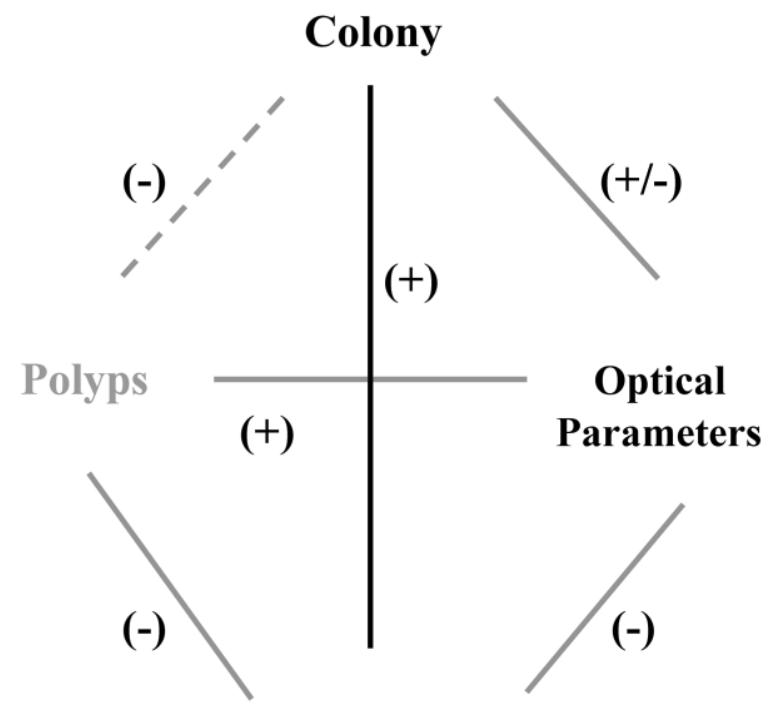

Sclerites

Figure 4.12. Phenotypic module integration models of Isis hippuris morphotypes from (a) Ridge, and (b) Sampela. Line key: ( $(-)$ very strong to strong correlation, ( $)$ weak to moderate correlation, $(--)$ ) and grey lettered modules are negligible, (+/-) positive/negative correlation.

$P<0.0001)$. Tests of within and between trait integration, which act to delimit I. hippuris morphotypes across sites, were modelled through covariance matrices (Table 4.9) corroborating vector values throughout the analyses. Phenotype module (colony, polyps, sclerites, and optical parameters) vertices were all significantly correlated within and between other modules with the consistent exception of polyps (Figure 4.12; Table 4.9). Polyps were the most weakly correlated trait, particularly at the Ridge, with those present typically responding differently, if at all, between the two sites/morphotypes to environmental change (polyps to sclerites and optical parameters). A similar scenario existed between colony responses and optical parameters with pigment densities positively correlated at Sampela. Colony level traits were moderately well correlated with sclerites regards both sites. Sclerites had a weak negatively correlation with chl $a$ pigmentation $[A]$ at Sampela and mitotic index $[\mathrm{MI}]$ at the Ridge, yet doubtful that such associations have any meaning in terms of integration. Typically stronger in Sampela chl $a$ cellular pigment density $[C Z]$ was positively correlated with $[A]$ yet both having a weak negative association with ZD, suggestive of self-shading. Generally the majority of correlations between phenotypic modules were weak with some in contrast which tends to suggest a lack of coordination in development and growth between the two morphotypes and limited integration between phenotypic modules. 


\subsection{DISCUSSION}

Previous research revealed that I. hippuris morphotypes within the WMNP were genetically segregated, strongly suggesting ecological divergence between healthy and anthropogenically impacted reefs (Chapter 3). Here, differential responses to environmental change illustrate phenotypic plasticity in healthy reef morphotypes, with reduced trait variability in colonies from the impacted reef, suggestive of genetic differentiation $\left(V_{G}\right)$ or genotype-by-environmental interactions $\left(V_{G x E}\right)$ between most traits. Specifically, colonies parsed annually primarily due to growth and source colony differences. With the exception of optical traits, all bathymetric differences were at the Ridge at the colony and chl $a$ absorbance levels. Irrespective of test, Sampela clones did not differ significantly between sites or depth; again with the exception of optical traits largely driven by chl $a$ absorbance levels. Most striking however was the lack of polyp, and to a lesser extent, sclerite differentiation between tests expressing trait canalisation particularly at the primary module level (polyps sensu stricto). Optical trait parameters were highly variable particularly in Ridge clones, yet Symbiodinium cladal type remained unchanged across all test colonies indicative of both plasticity capacity yet under some degree of host control. Trait integration was largely at the within phenotypic module level indicative of residual influences (e.g., developmental, trophic, and the cumulative effect of low variable traits) within and between traits. Such patterns coupled with known genetic differences and low dispersal properties (Chapter 3) make pertinent two notions; that I. hippuris morphotypes have 1) a high plasticity capacity as seen in Ridge colonies which, 2) over time through continual anthropogenic encroachment is resulting in incipient ecological divergence gradually leading to phenotypic canalisation at the module level in Sampela colonies. Therefore, two genetic and phenotypically divergent lineages partition at opposite ends of an environmental cline largely driven by differential light attenuation, hydrodynamics and poor dispersal, all likely exacerbated by anthropogenic influence at Sampela itself semi-lagoonal.

\subsubsection{Isis hippuris Plastic or Fixed?}

Results for the first objective demonstrate clearly that I. hippuris morphotypes within the WMNP show differential phenotypic responses in contrasting reef environments. Tests of local adaptation through reciprocal transplant experiments suggest phenotypically plastic colonies from the Ridge and incipient ecological divergence in Sampelan residents, the latter relatively insensitive to environmental change. Such patterns basically indicate that I. hippuris is inherently plastic, yet at Sampela may possibly have become functionally overwhelmed through prolonged anthropogenic disturbance leading to a breakdown in evolutionary capacitance (accumulated cryptic genetic variation). Fixation of adaptive cryptic variation over that of 
random mutations, lead to genetic assimilation of the adaptive phenotype through epigenetic heritability. Separate adaptive fitness peaks through ecological niche specialization start to emerge. Peaks become further reinforced by the actions of pleiotropy, linkage disequilibrium or concerted evolution (Sánchez \& Lasker 2003, Naidoo et al. 2013) as well as a reproductive strategy that overrides processes such as genetic drift and migration in an inherently plastic phenotype over time (Levene 1953, Hereford 2009, Blanquart et al. 2013). Thus, local adaptation at Sampela enhances diversity whereby selection in a novel environment may eventually supersede other evolutionary forces should conditions persist.

Resilience to environmental change will naturally be reflected in a population's ecology and survival, with habitat-dependent phenotypes showing immigrant inviability on transplantation (Prada \& Hellberg 2013). I. hippuris test clones from Sampela were robust to environmental change, suggesting that a robust phenotype may be evolving in, and to, an anthropogenically compromised environment. This scenario has been observed in other taxa (e.g., Barshis 2009, Bellantuono et al. 2012), whereby low cost adaptation is reflected in weak or absent functional trade-offs (Hereford 2009). Conversely, greater plasticity as seen in Ridge residents leads to functional trade-offs on transplantation to degraded shallow reefs irrespective of accidental human interference. Thus, even when controlled for competition through block elevation and clipping distance, I. hippuris phenotypes from the Ridge have greater habitat dependency for shallow clear water reefs, exhibiting less survivorship under environmental change. Divergence in trade-off necessity between morphotypes, therefore provide further evidence for the onset of ecological speciation (Rundle \& Nosil 2005) with Sampela residents relatively resilient to environmental change.

Plasticity capacity is shaped by natural selection leading to genetic stability through genetic assimilation in a stress-induced phenotype and may further lead to genetic incompatibility with non-native I. hippuris corals. However, this idea appears unlikely at this point, due to the presence of eggs on disturbed transplanted clones at Sampela from the Ridge. Test colonies were externally brooding pink eggs, also observed in the surrounding native colonies, but not present on either test control or colonies within the area. This suggests the presence of waterborne exudates inducing coordinated reproductive activity between colonies only at Sampela and that such exudates remained functional at the induction level. However, post zygotic isolation between source and transplanted colonies is still not out of the question, warranting further investigation. Nonetheless, it may be interpreted that resources in the clippings on the disturbed block, were allocated to reproduction for survival whereas all other 
test colonies were in a state of juvenile growth - note that the presence of eggs in 2010 source colonies discounts the notion that all were male. Time to sexual maturity in these animals is unknown, however it is not unlikely that sexual maturity is typically limited to $\sim 20 \mathrm{~cm}$ in height, previous years being dedicated to growth as seen in other gorgonians (e.g., Brazeau \& Lasker 1990, Coma et al. 1995, Beiring \& Lasker 2000) in order to establish the colony.

\subsubsection{Isis hippuris Holobiont Responses}

For the second objective, tests of divergence through local adaptation using reciprocal transplant experiments, demonstrated differential physiological responses of I. hippuris morphotypes within the WMNP in contrasting reef environments. Annual growth accounted for multivariate model differences, yet when adjusted for positive association, revealed clear colony (viz., branching) plasticity at the Ridge particularly across bathymetry. Reductions in colony porosity, branch articulation (TB\#, Bu\#), and chl $a$ absorptance $(A, C Z)$ with increased branch length primarily in transplants to Sampela maximizes light capture, reducing self-shading as well as sediment settlement typical of low light and water flow environments (Kawaguti 1943, Shaish et al. 2006). Functional integration at the morph-optical trait level is only moderately correlated, however, opposed between I. hippuris morphotypes. Similarly, polyp density was only weakly correlated with optical traits at Sampela, with a slight reduction on transplantation to the Ridge, which may be concomitant with reduced allochthonous food sources and/or differential light availability. Low polyp densities are typically encountered in low light (as a function of depth) environments (West et al. 1993, Kim et al. 2004, Prada et al. 2008, Prada \& Hellberg 2013), possibly a regulatory mechanism to reduced resource availability. Overall, fine-scale differences within polyp and canal dimensions, particularly at depth, with concomitant variations in zooxanthellae and pigment densities, are further indicative of dynamic shifts in resource reliance at the optical and colony level facilitating the additive effect of polyps (module sensu stricto) as a growth response and primary resource facilitator.

Colony growth and form are dependent on feeding strategy, with the same genotype often showing differential resource allocation patterns (hetero/phototrophic capacity) in different environments (Sebens 1997, Poorter \& Nagel 2000, Weiner 2004). The relative dependency of phototrophy and heterotrophy in I. hippuris morphotypes in the different environments have, however, likely shifted without significant changes in endosymbiont or, as in the case of Sampela, phenotype. Previous work revealed species specificity in octocoral heterotrophic food source with I. hippuris from the Seychelles and the Great Barrier Reef only feeding on bacterioplankton and curiously low photosynthetic rates (Sorokin 1991). This implies minimal 
resource translocation, yet results presented here are in agreement with bio-optical and biophysical values for other cnidarian taxa (e.g., Falkowski \& Dubinsky 1981). Moreover, holobiont metabolism can be profoundly affected by light (Baker et al. 2011), sedimentation (Riegl \& Branch 1995) and nutrient enrichment (Risk et al. 2009), which often act in exacerbated concert (e.g., Baker et al. 2011).

The sclerites of gorgonian corals are suggested to be most susceptible to selection through environmental change (Bayer \& Stefani 1987, West et al. 1993, West 1997, Prada et al. 2008; but see Skoufas 2006). Here, only subtle variations in coenenchyme surface and subsurface capstans and spindles respectively occurred within the test period. Notably, the sclerites of the Sampelan clones remained consistently smaller in size reducing further at the Ridge. A decrease in calcification through reduced photosynthate translocation may account for this growth reduction in Sampela transplants to the Ridge, in addition to a greater former reliance on heterotrophic feeding through nitrogenous and allochthonous sources at Sampela. Utilization of various nitrogenous sources (e.g., nitrate, nitrite, ammonium) can also increase zooxanthellae densities (Muscatine et al. 1989, Fagoonee et al. 1999) and subsequent fixed carbon production through increased photosynthetic efficiency (Koop et al. 2001, Lesser et al. 2010). Furthermore, nitrogenous compounds stimulate calcification with or without light (Crossland \& Barnes 1974). Thus the mutual exchange of photosynthetic carbon and nitrogen (whether from the host and/or eutrophic sea water) likely act synergistically in the formation of skeletal elements and growth, which may account for reduced sclerite growth when removed from high nutrient waters in adapted I. hippuris phenotypes. Therefore, assessing energy apportionment within and between I. hippuris morphotypes, endosymbionts, and the environment using comparative stable isotope analyses $\left(\delta^{13} \mathrm{C}\right.$ and $\left.\delta^{15} \mathrm{~N}\right)$ would greatly elucidate energy transfer roles and thus, differential trophic allocation as a mechanism of phenotypic plasticity and divergence to environmental change.

The consistency in reduced sclerite size of and to Sampela may be host regulated, enhancing the internal light field under reduced pigment density in order to maintain overall absorption efficiency (Schlichter et al. 1986, Stambler \& Dubinsky 2005, Enríquez et al. 2005). Dynamic stability was further established in Sampela colonies with evident adjustments in Ridge transplants to Sampela. Moreover, the effective quantum yield ( $\Delta \mathrm{F} / \mathrm{Fm}$ '), expressing the variation in PSII photochemical efficiency under ambient light (in situ; the capacity to use the absorbed light energy), was relatively invariable with slight increases in colonies transplanted to and from Sampela. Assessing light-adapted fluorescent yield is on an immediate time scale and 
does not depict reaction centre dynamics which may be disrupted mirroring the $>50 \%$ pigment loss in Ridge transplants to Sampela (see Dove et al. 2006). Therefore, to assess light absorption efficiency and utilization (= photosynthesis), in situ rapid light response vs. irradiance (PAR) curves would reveal if morphotypes differentially modify non-photochemical quenching, maintaining constant photoacclimatory operating efficiency in their respective environments (Hennige et al. 2008).

All test colonies exhibited identical patterns in zooxanthellae density, specifically reductions with increased bathymetry irrespective of site. This suggests regulation through irradiance and anatomical-specific carrying capacity particularly considering a concomitant reduction in colony growth and area with depth, also previously noted in scleractinians for seasonal irradiance (Fitt et al. 2000, Warner et al. 2002) and nutrient shifts (Fagonee et al. 1999). Typically, photoacclimatory responses to heterogeneous irradiances involve variations in chl $a$ concentrations at the cellular level (Falkowski \& Dubinsky 1981, McCloskey \& Muscatine 1984, Porter et al. 1984, Fitt \& Cook 2001). In this study, chl $a$ absorbance efficiencies $(A, C Z)$ were positively correlated. Characteristic increases in light absorption efficiency via greater pigment density at depth (McCloskey \& Muscatine 1984, Porter et al. 1984, Enriquez et al. 2005, Lesser et al. 2010) were evident only in Ridge residents, the inverse being true in Sampela clones, further homogenised at the cellular level indicative of photoadaptation over time. Interestingly, however, Ridge transplants to Sampela revealed clear photoacclimatory responses to variable irradiance levels through both optical and morphological trait adjustment. Increased branch and chl $a$ per cell densities are typical photoacclimatory responses to increased light attenuation in Scleractinia (Falkowski \& Dubinsky 1981, McCloskey \& Muscatine 1984, Porter et al. 1984), yet the opposite was largely true for these gorgonian Ridge transplants. A combination of reduced branch density and $>50 \%$ reduction in chl $a$ concentration indicate stress responses to increased irradiance. But a reduction and maintenance in chl $a$ densities per cell in shallow and deep test colonies respectively, concomitant with increased total branch length and overall reduced articulation suggest stress responses to increased irradiance are either masked or exacerbated by elevated nutrient levels and sedimentation at Sampela. Nevertheless, absorbance through pigment density at Sampela may indicate the onset of genetic accommodation demonstrated by an evolutionary shift in slope elevation of the reaction norm (Aubin-Horth \& Renn 2009). Photoacclimatory control within the I. hippuris holobiont may therefore lie to some degree with the host particularly considering enhanced physiological performance in free-living Symbiodinium (e.g., Enríquez et al. 2005). However, photoacclimation in effective antennae-absorption is relatively conserved across certain 
Symbiodinium cladal types, modifications present at the biophysical level (A - B, F; Hennige et al. 2009). Whether this is the same for clade $D$ variants is unclear requiring investigations into PSII light curve interception to elucidate if endosymbionts within I. hippuris morphotypes differentially modify non-photochemical quenching to maintain constant photoacclimatory operating efficiency in alternate environments (see Hennige et al. 2008). The dynamic interplay between host-symbiont regulatory roles leading photoacclimatory capacity within the holobiont is fascinating (reviewed by Yellowlees et al. 2008, Davy et al. 2012, Fay \& Webber 2012, Lesser et al. 2013) yet outside the scope of this study.

Photoacclimation combining optical and morphological traits can be seen in the increase of $a *$ in Ridge clones when transplanted to Sampela. Here, results strongly suggest that multi-scattering of the local light field and enhanced incident radiation capture were facilitated through the intricate geometry of the sclerites and axis. This down regulation of light absorption efficiency through reduced pigment density (Enriquez et al. 2005), coupled with increased zooxanthellae cell division (MI) is indicative of increased nutrient load and heterotrophic uptake (Fitt \& Cook 2001, Davy et al. 2012). The generally small ( $c f$. Wilkerson et al. 1988) zooxanthellae cell size and SA:V in Ridge colonies compared to Sampela started to increase in size on transplantation, emulating that of Sampela residents. Such subtleties maybe supportive of more variable trait responses, or may simply reflect increased intracellular space through reduced symbiont cell density at Sampela. Cell division and size vary with depth and coral taxa, previously negatively correlated (Wilkerson et al. 1988); yet the opposite is the case in I. hippuris. Symbiodinium cells between host morphotypes were consistently 5\% larger within the Sampela holobiont but reduced to $<1.4 \%$ difference due to an increased cell size in Ridge transplants to Sampela, suggesting non-heritability within the Ridge. However, heritability or the onset of is unlikely in Sampela clones - contrasted with those noted in Jamaican scleractinians (Schoenberg \& Trench 1980) - considering the invariable Symbiodinium cladal types across all test taxa. Taken together, Sampela clones showed a repetitive level of dynamic stability in optical and overall phenotypic traits across all tests, delimiting them from Ridge colonies. Photoacclimatory responses at the physiological and morphological level facilitated Ridge colonies at Sampela, likely unaccustomed to high nutrient, sedimented and low water flow environments further exacerbated by variable irradiance levels interacting with the light absorbing and scattering properties of the water itself (Stambler \& Dubinsky 2005) and its elevated particulates (Porter 1976). 


\subsubsection{Endosymbiont Specificity}

Resource allocation structure within the I. hippuris holobiont maximises fitness through physiological optima set by a novel Symbiodinium clade D1a. Technological advances reveal multiple cryptic endosymbiont types providing acclimatory 'flexibility' to environmental change in 39 scleractinian species (Silverstein et al. 2012 see also Putnam et al. 2012). Yet clear coralalgal specificity within the I. hippuris holobiont was evident with little genetic variation across highly variable (ITS2, psbA) and relatively conserved (COX1) molecular markers. Hostsymbiont specificity is not uncommon in Octocorallia (Goulet \& Coffroth 2003, van Oppen et al. 2005, Goulet 2006, Goulet et al. 2008, Baker \& Romanski 2007) and has been attributed to an assumption of reduced autotrophic dependence within this group (Baker \& Romanski 2007, but see Sorokin 1991). As stated, relative trophic structure is undetermined in I. hippuris colonies within the Wakatobi, however harbouring putatively stress tolerant clade D1a Symbiodinium (Jones et al. 2008, LaJeunesse et al. 2009, Stat \& Gates 2011, but see Abrego et al. 2008) likely adds to the holobiont biological success, host-symbiont phylotype specialists apparently the most resistant to environmental stress (Putnam et al. 2012). Contrary however, is the currently perceived opportunistic (Stat \& Gates 2011) or parasitic (Sachs et al. 2011, Lesser et al. 2013) nature of clade D. Tests of Symbiodinium diversity and reciprocal metabolic pathways within the holobiont using next generation sequencing and predictive modeling (PRMT; Larsen et al. 2011a) would determine both cryptic symbiont communities and metabolic interactions that structure holobiont trophic adaptations across environmental gradients. Resulting metabolic hypotheses may then be addressed through metabolic profiling, providing strong insights into integral metabolic links within the coral-Symbiodinium symbiosis, further elucidating nutrient cycling and the role of endosymbiont(s) in response to environmental change (Larsen et al. 2011b).

\subsubsection{Isis hippuris Trait Integration}

In order to test the theoretical framework of local adaptation leading to divergent selection through the action of environmental perturbation, the suite of phenotypic traits considered were summarised into phenotypic modules with topological equivalent traits driving the overall distributions determined. In the final objective, significant trait integration was greatest within, yet low between, phenotypic modules, likely indicative of residual errors $\left(V_{\text {error }}\right)$ through developmental, trophic, and the cumulative effect of low variable traits. These factors as well as optical dampers such as mycosporine-like amino acids (MAAs; Gates et al. 1995, Lesser \& Farrell 2004) and green fluorescent proteins (GFP; Dove et al. 2006) may also contribute to the residual variance (Hageman et al. 1999) between phenotypic correlates. Furthermore, 
developmental covariance between traits, coupled with the action of pleiotropy can lead to covariance of seemingly unrelated traits. Tests of conditional dependence/independence (partial correlation coefficients; Magwene 2001, Sánchez \& Lasker 2003, Wille \& Bühlmann 2006) may yield insight into the relative integration within and between phenotypic traits and how they associate under environmental change. However, as evident in tables 4.4 - 7, 4.9 and respective phenotype module figures, the collective effect of each trait likely pull together even in synergy to determine the overall phenotype at any one point in time and space (Blanquart et al. 2013). Thus, an integrated trait cascade effect may act in unison - it is of interest that omission of primary traits on the same multivariate modules yield identical results. Hence, such traits may act as facilitators, by-products of the main adaptive traits or an interaction of the two, the former consequential of the latter (Gould \& Lewontin 1979). In sum, trait integration was typically higher within Ridge sourced colonies indicating greater plasticity through integration - a necessary component for rapid responses to environmental change. Yet phenotypic invariability was evident in Sampela clones pre-adapted to variable light and high nutrient loads.

It is essential to distinguish between the 'primary adaptive' and 'by-product' traits; a relatively low/invariant trait may in fact be the adaptive framework of which an organism is constructed. In the case of I. hippuris - and gorgonians per se - it is clear that polyps are the primary adaptation, considered canalised with only their variable profusion indicative of environmental influence. Phenotypic characters such as the coenenchyme (soft tissue), sclerites, and axis etc. have in fact originated from the polyp wall, are by-products and subsequent facilitators of the primary modules, the polyps and thus coined 'exaptations' (evolved not for their original utility; Gould 1997). Yet selection acts on plasticity capacity (Kaandorp \& Kübler 2001) resulting in divergent phenotypes often evident at the phenotypic level with favoured (often facilitative/byproduct) traits the subject of evolutionary change (Kawecki \& Ebert 2004). It can therefore be considered that by the interaction of light, nutrient and hydrodynamic regime, branching dynamics are simply 'exaptive' (Gould 1997) or the platform for polyps, the primary adaptation. The developmental influence of polyps on the colony therefore, should be the research target for processes of evolutionary change. Theoretical supposition aside, evolutionary changes on the phenotypic level are nature's manifesto of how human influences are affecting evolutionary change, which in itself will continue to persist irrespective of our presence.

\subsection{CONCLUSION}

Reciprocal transplant experiments across contrasting reef sites within the WMNP, strongly suggest plasticity capacity and incipient divergence in phenotypic traits for Ridge and Sampela 
I. hippuris morphotypes respectively. I. hippuris morphotypes present at the Ridge and in previously sampled locations within the region (Chapter 3) are therefore, the ancestral type. Tests of coalescence would provide validation to this hypothesis (Puritz et al. 2012). Patterns of divergence suggested also by immigrant inviability, are likely determined through prolonged anthropogenic disturbance underpinned by reproductive strategy, which overrides processes such as genetic drift in an inherently plastic phenotype (Levene 1953, Hereford 2009, Blanquart et al. 2013). Resource allocation structure within the holobiont likely reaches differential physiological optima for each I. hippuris type, which maximises fitness in their respective environments through morph-optical trait integration. Both morphological and physiological photoacclimatory responses confirm mechanistic adjustments to maintain such fitness optima. Multivariate models reveal polyp dynamics being largely canalised and determinate traits; the density and size of which controlling resource acquisition and capacity (in terms of endosymbiont density). Investigations into the relative dependence on autotrophy verses heterotrophy leading to resource allocation change within the I. hippuris holobiont would undoubtedly confirm mechanisms of both biological success and incipient divergence consequential of genetically assimilated phenotypes to environmental change. It is proposed that I. hippuris morphotypes are in a state of ecological divergence and act as viable indicators on the effects of burgeoning anthropogenic encroachment on mechanisms of biodiversity and reef health within the Coral Triangle. 


\section{CHAPTER 5: GORGONIANS IN THE WAKATOBI MARINE NATIONAL PARK, INDONESIA: WHAT CAN THEY TELL US ABOUT EVOLUTIONARY PROCESSES IN ENVIRONMENTAL CHANGE?}

\subsection{CONTEXTUAL SUMMARY}

The Wakatobi Marine National Park (WMNP), SE Sulawesi, Indonesia, comprises possibly the most biodiverse marine ecosystems on the planet with gorgonian corals epitomising such diversity. Central within the Coral Triangle, the WMNP's human population and inherent exploitations are as diverse as its natural resources. In fact the natural laboratory this region presents, encompasses natural and anthropogenic interactions bestowed upon the researcher as a haven for discovery and local collaborations akin to those by the marine fauna and flora itself. This work clearly demonstrates the utility of gorgonian corals as conservation indicator taxa through straightforward ecological assessment and experimentation, specifically across environmental clines.

As conspicuous members of any marine community, the modular nature of gorgonians continues to baffle scientists. Still, unresolved questions of plasticity, divergence, or homoplasy hamper species delimitation and biodiversity assessments, particularly on coral reefs throughout the Indo-Pacific (van Ofwegen 2004) despite their high regional abundance and diversity (Tomascik et al. 2004). The present work substantiates these notions, whereby 197 species and morphotypes from 42 genera, and 12 families within all currently accepted suborders were recognised from the shallow waters of the WMNP, increasing with depth inviting tests of refugia (Chapter 2). Meticulous morphological differentiation is not, however, indicative of noninterbreeding taxa, and may in fact represent differential phenotypic responses to environmental heterogeneity.

Assessment of gorgonian abundance and diversity across environmental gradients within the WMNP revealed a clear loss of gorgonian diversity relative to increased sedimentation and reduced light associated with anthropogenic disturbance. Zooxanthellate and azooxanthellate taxa were clearly parsed between depth and reef health, the former more tolerant to anthropogenic perturbation than the latter. Notably, the two distinct morphotypes of the zooxanthellate isidid Isis hippuris Linnaeus 1758, were highly abundant across environmental clines: long-branched bushy colonies on degraded reefs, and short-branched multi/planar colonies on healthy reefs. Such morphological differentiation may be a consequence of high 
plasticity capacity of the I. hippuris holobiont, two previously diverged species, or anthropogenically driven incipient ecological divergence on degraded reefs. Morphological and molecular results reveal unsatisfactory assignment of $I$. hippuris morphotypes to previously described alternatives (Isis reticulata Nutting 1910, Isis minorbrachyblasta Zou, Huang \& Wang 1991), further suggesting incipient ecological divergence through clear haplotype division between sites of differing reef health (Chapter 3). Multivariate analyses consistently revealed light availability, sedimentation and water flow as significant explanatory variables for morphotype differences, suggesting a dynamic interplay between I. hippuris morphotypes and their environment. To further assess potential mechanisms of divergence and survivorship in alternate reef habitats, a reciprocal transplant experiment (RTE) for one year yielded insightful results (Chapter 4). Firstly, reduced survivorship of healthy reef morphotypes on degraded reefs implied the onset of lineage segregation through immigrant inviability. Secondly, multivariate analyses revealed differences were attributed to I. hippuris morphotype origin, with phenotypic responses to environmental change typically plastic in colonies from the healthy site, whereas those from the degraded site were relatively insensitive to change. Prominent phenotypic traits were at the morphological and bio-optical levels integrated to maintain functional optima, ultimately influenced by resource availability and acquisition. Thirdly, that such optical responses were not attributed to endosymbiont diversity or shuffling, with all test colonies possessing a novel clade D1a Symbiodinium throughout. Residual error $\left(V_{\text {error }}\right)$ unaccounted for by measured phenotypic traits, invite tests of energy transfer roles within and between the holobiont including biophysical photoacclimatory responses assessing functional thresholds to environmental stress.

This research aimed to investigate gorgonian responses to environmental change within the WMNP, SE Sulawesi, Indonesia. Collectively, this was achieved, challenging notions of ecological importance, taxonomic validity, and the overall effects of anthropogenic encroachment on mechanisms of plasticity and divergence within the coral holobiont. Key issues as a consequence of this work include tests of 1) deep-reef refugia, 2) priority systematics, and 3) mechanisms of ecological divergence and physiological assessment exploring intrinsic and extrinsic interactions that may define the host-symbiont relationship.

\subsection{BIODIVERSITY \& REFUGIA}

Gorgonian corals within the WMNP exhibited high species diversity and abundance particularly on healthy coral reefs (Chapter 2). Of the 51 genera and 14 families recognised for shallowwater gorgonians in the Indo-Pacific (Fabricius \& Alderslade 2001), 42 genera and 12 families 
were present within the WMNP, comprising all higher order groups. Coral reef biodiversity is considered at its peak within the Coral Triangle, most notably in the Indo-Malay-Philippine (IMP) region (Stehli \& Wells 1971, Veron et al. 2009, 2011, Bellwood et al. 2012, Briggs \& Bowen 2013, Gaither \& Rocha 2013). The origin of such diversity remains the source of much intrigue and investigation with various hypotheses being proposed. Firstly, the Centre of Origin hypothesis predicts the IMP as a centre of speciation where species disperse marginally (Ekman 1953). Secondly, the Centre of Overlap hypothesis predicts species diversity to be a consequence of dispersal overlap in all directions from numerous biogeographic provinces (Woodland 1983). Thirdly, the Centre of Accumulation hypothesis predicts that peripheral speciation through dispersal is extended unidirectionally by prevailing currents into the IMP (Ladd 1960), and finally, the Centre of Survival hypothesis whereby species are buffered by extinction in contrasting peripheral locations (Paulay 1990). All likely pull in unison, with differential responses of marine taxa and biodiversity feedback between hypothesised models, attributing to the current high biodiversity (Bowen et al. 2013). Yet how such hypotheses stand in the face of anthropogenic impact accelerating and/or exacerbating natural processes of environmental change is unknown. Furthermore, such hypotheses are almost solely based on reef fish and scleractinian corals (see Bellwood \& Hughes 2001 but now see Bowen et al. 2013, Sanciangro et al. 2013) advocating latitudinal gradients of species richness driven by the universal currency of energy availability (Gaston \& Spicer 2004, Evans et al. 2005). In its simplicity, most biodiversity may be a synergistic combination of increased habitat (Sanciangro et al. 2013) and energy/resource availability (Rohde 1992). Yet how these hypotheses relate to gorgonian corals without greater sampling effort is unknown, further exacerbated by a paucity of knowledge on gorgonian reproductive strategies throughout the Indo-Pacific, casting doubt on dispersal ability, range size and subsequent taxonomic assignment. Nevertheless, comparative global coral diversity primarily lies within the Octocorallia, comprising estimates of $64 \%$ (3400 species) compared to that of Scleractinia 27\% (1450 species; Williams \& Cairns 2013). Moreover, gorgonian corals are phylogenetically older than the Scleractinia (Lindstrom 1978, Bengtson 1981, Cope 2005, Stolarski et al. 2011). Therefore, as one of the most diverse invertebrate groups in benthic marine ecosystems, typically conservation 'flagship' species (Tinsley 2005, Linares et al. 2008, Cerrano et al. 2010) in many regions, it is curious that gorgonians are generally overlooked on coral reefs within the Indo-Pacific.

In the WMNP, gorgonian diversity increases with depth, almost exclusively by azooxanthellate taxa (Chapter 2). Such diversity, colony size and number of recruits were observed to 


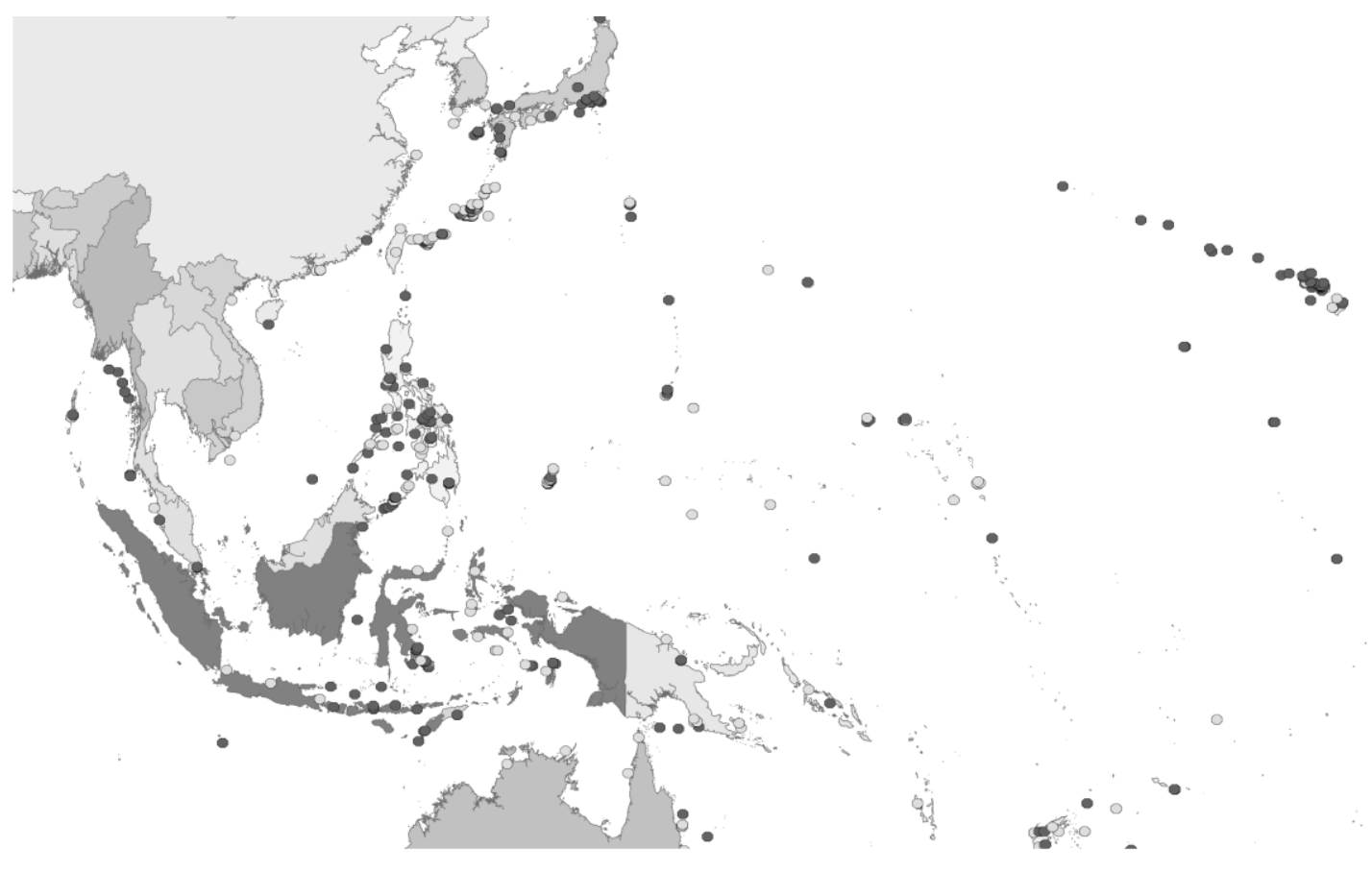

Figure 5.1. Distribution of Indo-Pacific tropical gorgonians found at shallow ( 0 - $39 \mathrm{~m}$; green spots) and mesophotic (40 - $200 \mathrm{~m}$; blue spots) depths. Data sources: USNM, AMNH, BPBM, NIWA, Nutting (1910a - e, 1911), Stiasny (1937, 1940), Mai-Bao-Thu \& Domantay (1970, 1971), Muzik \& Wainwright (1977), Colin et al. (1986), Goh \& Chou (1994, 1996), Paulay et al. (2003), Fabricius et al. (2007), Rowley (2013; Chapter 2).

continually increase to depths way beyond $60 \mathrm{~m}$ (pers. obs.). This pattern is consistent with other Indo-Pacific regions where benthic communities become dominated by gorgonian corals

at 40 - 200 m (Figure 5.1; Marshall Islands, Colin et al. 1986; Palau, Mariana Islands, Paulay et al. 2003; Palau, Fabricius et al. 2007; Great Barrier Reef, Australia, Bridge et al. 2012; Philippines 2013, Rowley unpublished data). Deeper reefs ("mesophotic coral reefs" [MCEs] or the "twilight zone") are posited to act as refugia against disturbances as well as a haven for larval source pools (Glynn 1996, Reigl \& Piller 2003, Bongaerts et al. 2010). Most importantly, such deep reef refugia are hypothesised to harbour reef components largely unaffected by geological sea-level change during times of markedly reduced shallow water habitats (Helm \& Schülke 2003; RL Pyle, BW Bowen \& J Copus pers. comm.). Increasing evidence reveals deep reefs possess unique communities with less than 50\% overlap of shallow taxa (Pyle 1988, 1990, Macintyre et al. 1991, Bongaerts et al. 2010). In the WMNP, gorgonian diversity increased with depth, with numerous taxa distributed from $5>60 \mathrm{~m}$, a pattern consistent with records from other Indo-Pacific regions (USNM, BPBM, BNHM, Nutting 1910a - e, 1911, Stiasny 1937, 1940, Mai-Bao-Thu \& Domantay 1970, 1971, Paulay et al. 2003, Fabricius et al. 2007). 
Overlapping taxa across bathymetry are ideal targets for tests of resilience and comparative divergence through local adaptation within certain genera, as well as 'twilight' habitat specialists. Interestingly, azooxanthellate gorgonian genera such as Acanthogorgia Gray 1857, Annella Gray 1858, Bebryce Philippi 1841, Ellisella Gray 1858, and Villogorgia Duchassaing \& Michelloti 1862 span remarkable depths $(5 \geq 1000 \mathrm{~m})$ across their distributional range. Yet within-group polyphyly across bathymetry (deep-water monophyly typically disrupted by shallow-water taxa; McFadden et al. 2006, Pante et al. 2012, Chapter 3) calls for further systematic assessment (e.g., coalescence; Puritz \& Toonen 2011, Puritz et al. 2012b), with polyphyletic groups either a consequence of convergent evolution or deep divergence.

The question of shallow reefs becoming seeded by deep reefs is determined by species-specific responses to intrinsic (e.g., reproduction, settlement, development, symbioses) and extrinsic (e.g., hydrodynamics, light, temperature, sedimentation, water quality) factors (Fabricius et al. 2007), as well as evolutionary processes such as speciation, extinction and dispersal (Mora et al. 2003). Determining how and whether certain taxa are habitat specialists or bathymetric migrators remains to be elucidated, undoubtedly unveiling key evolutionary mechanisms facilitating survival over geological time. Moreover, assessing evolutionary principles on taxa inhabiting mesophotic reefs may shed light on the origin of deep-sea and shallow-water taxa. Before the opening of the Drake Passage 25MYA (late Eocene) and the onset of the thermohaline circulation, much of the deep oceans were decidedly warmer and often anoxic (Chase et al. 1975, Scher 2006). Thus, the deep-sea gorgonians we know today would have migrated and evolved from shallower depths with huge depth ranges still apparent (e.g., > 4000 $\mathrm{m}$ range in deep-sea Chrysogorgiidae; USNM records 2013). Naturally, such benign marine environments invite deep-sea specialists such as Metallogorgia melanotrichos Wright \& Studer 1889, with just a single haplotype across its geographic range of three oceans (Pante et al. 2012), similarly with its associate ophiuroid within the Atlantic (Cho \& Shank 2010). Conversely, in the shallows, environmental heterogeneity selects high plasticity capacity that may become fixed at functional optima (as suggested in Isis hippuris at Sampela in Chapter 4), leading to restricted geographic range size and endemicity (e.g., Calosi et al. 2008, 2010).

Clearly, a high number of gorgonian taxa are widely distributed throughout the Indo-Pacific is present, particularly at mesophotic depths (Figure 5.1). Such unique communities may be relatively consistent throughout the Indo-Pacific and particularly prevalent within the Philippines (Figure 5.1) consisting of over 7100 islands (Williams \& Chen in review) likely acting as habitat stepping-stones for dispersal within and between this and other regions. This, 
coupled with ecoclimatic stability within the Coral Triangle, provides testable hypotheses for the distinct gorgonian diversity as shown in the WMNP and other regions (Colin et al. 1986, Paulay et al. 2003, Fabricius et al. 2007, Bridge et al. 2012, this study). Nevertheless, many regions require greater sampling efforts across bathymetry using advanced rebreather diving technology (e.g., Pyle 1996, Pyle et al. 2008) in order to begin addressing mechanisms of diversity and refugia. However, tests of diversity and evolution without historical investigation are inconclusive (Gould 1997, Rohde 1999), further requiring tests of coalescence.

\subsection{SYSTEMATICS IN THE SEA}

Ecological and taxonomic investigations indicate much work has yet to be completed to achieve effective gorgonian taxonomic assignment, in terms of conservation biodiversity assessments. Nevertheless, indicator taxa can and have been teased out as surrogates for reef component responses to environmental change (Chapter 2). In the case of Isis hippuris, gaining an historical perspective in the context of a structure-function relationship with its environment may provide insight into evolutionary divergent mechanisms between morphotypes (see Chapter 4). It is interesting, however, that phylogenetic analyses reveal that the Isididae is polyphyletic with the addition of its type species I. hippuris, and therefore seemingly unrelated (Chapter 3; Figure 3.7). The jointed bamboo-like axis (Figure 5.2a, b) that unites the family may simply be a relictual anachronism consequential of geological processes over time, yet phylogenetic analyses corroborate the early taxonomic suggestion that the characteristic jointed axis of the Isididae is in fact a convergent trait. Again, tests of phylogenomic coalescence using next generation sequencing (NGS) would differentiate evolutionary mechanisms acting between members within the Isididae; testing the evolutionary origin of the axis as either 1) deep divergence or, 2) convergent evolution. However, what would be the purpose of a jointed axis in the deep ocean when structurally there is no significant requirement for hydrodynamic flexibility in such a benign environment? A relictual anachronism therefore seems innately plausible with the evolution of flexibility through ecological necessity in shallow and steep wall environments, the latter often subject to high nutrient deep-water upwellings as seen at the healthy Ridge site in the WMNP (Gieskes et al. 1988). Even though unknown, the stratigraphic history may therefore evolve from either the high hydrodynamics on shallow reefs and/or refugial depths (e.g., 40 $200 \mathrm{~m}$ ), which then migrate through bathymetric expansion in response to resource competition, predation, random or specific settlement cues, encompassing any number or combination of ecological or developmental processes. Whatever the scenario, survival requires an individual and/or species to have the capacity to respond, and thus, not be entirely passive to its 

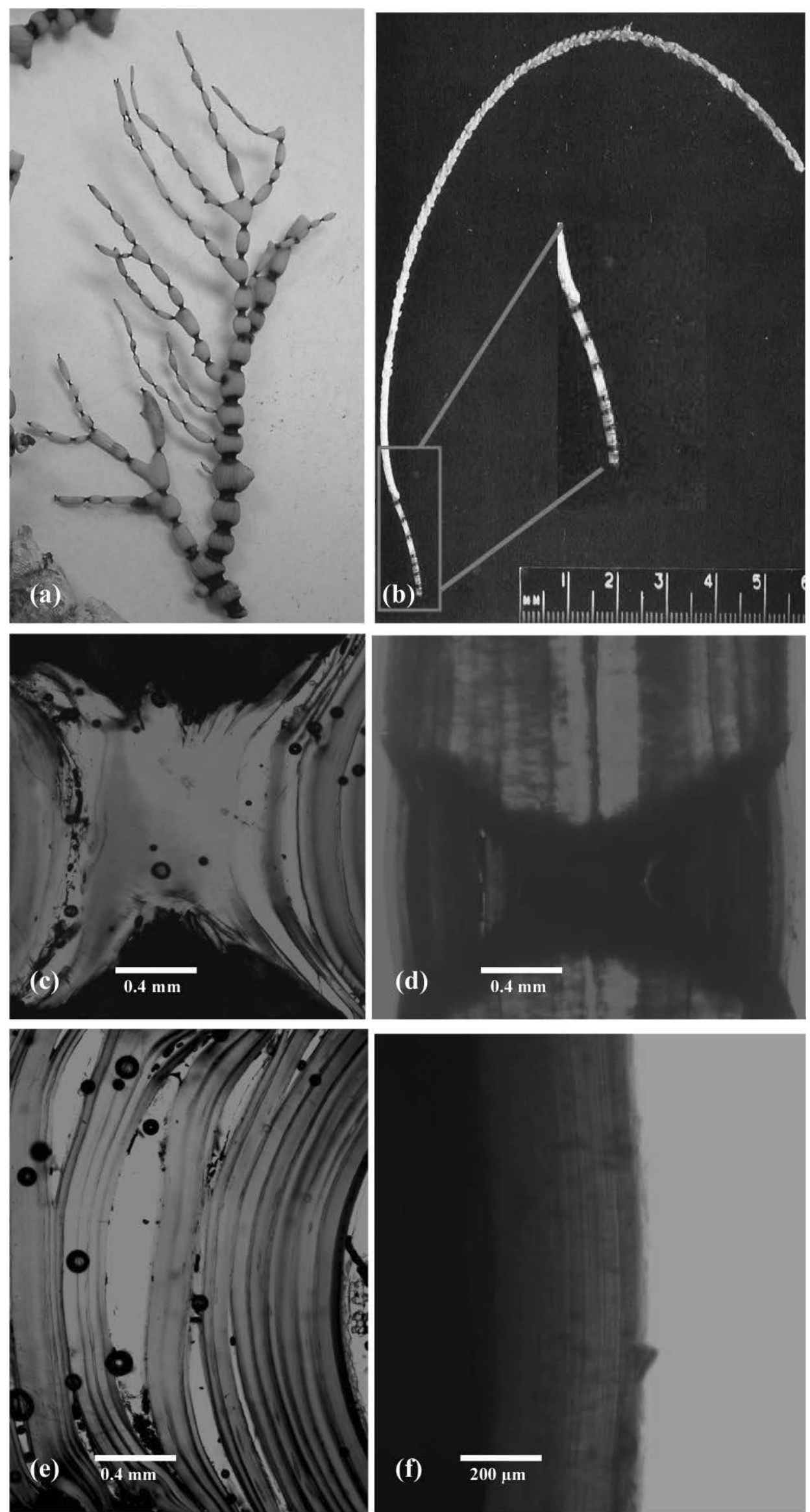
Figure 5.2. (Previous page) Isididae jointed axis comparison between Isis hippuris Linnaeus 1758 and Circinisis circinata Grant 1976 of the (a, b) denuded colony, (c, d) horny proteinaceous (gorgonin) node, and (e, f) node fibres.

environment, which is particularly true for those taxa which are sessile (Cossins et al. 2006). Migration to the shallows necessitates functionally advantageous traits to increases in light, hydrodynamics and temperature, including shorter more flexible axis joints (Figure 5.2c,e; Helm \& Schülke 2003) and photosynthetic endosymbionts as seen in Isis hippuris. Conversely, deepwater migration particularly in the advent of the thermohaline circulation (Chase et al. 1975, Scher 2006), facilitates expansion into benign environments whereby flexible widely spaced and fibrous proteinaceous horny nodes become taught and robust (Figure 5.2d, f) and often reinforced with calcareous material (Grasshoff \& Zibrowius 1983, Helm \& Schülke 2003). Clearly, isidids are functionally adapted to differential hydrodynamic regimes across bathymetry, however this does not explain the significant polyphyly within the family.

The consistent phylogenetic incongruence at the subordinal and family-levels (McFadden et al. 2006, Pante et al. 2012, Sanchez et al. 2003b) seemingly lacks functional divergence to environmental change. Fixed traits when redundant (such as a jointed axis in the deep-ocean) may well be maintained through continuous sea level changes over geological time; bet hedging with insurance if you will. Furthermore, a loss and re-gain of traits may occur through the differential expression of genes in response to environmental change over the millennia. It lacks empirical sense to have the repetitive evolution of multiple traits in the absence of any functional cause, however. For example, the fused scleritic composition of the calcareous internodes of $I$. hippuris contrasted with the non-scleritic axis of deep-sea isidids, characteristic of the Calcaxonia per se, is another logical trait differentiation parsing the Isididae across bathymetry (Figure 5.3). Clearly a scleritic axis would permit greater flexibility in high water flow environments, however numerous deep-sea taxa, namely of the Scleraxonia (e.g., Corallium Cuvier 1798 [see Figure 5.3b], Paragorgia Milne-Edwards 1857, Anthothela Verrill 1879) possess this same trait. Furthermore, the only exception to the Holaxonian axis composition of a horny axis supported by non-scleritic calcareous material including a central core is the Keroeididae Kinoshita 1910, notable again for its scleritic axis. Bathymetric distribution within the Keroeididae are again akin to previous adaptation to high flow shallower waters with subsequent bathymetric migration to safer depths e.g., Keroeides Studer 1887 of 51 $>650 \mathrm{~m}$, similarly with Corallium $96>2500 \mathrm{~m}$. Henceforth, such patterns provide target 

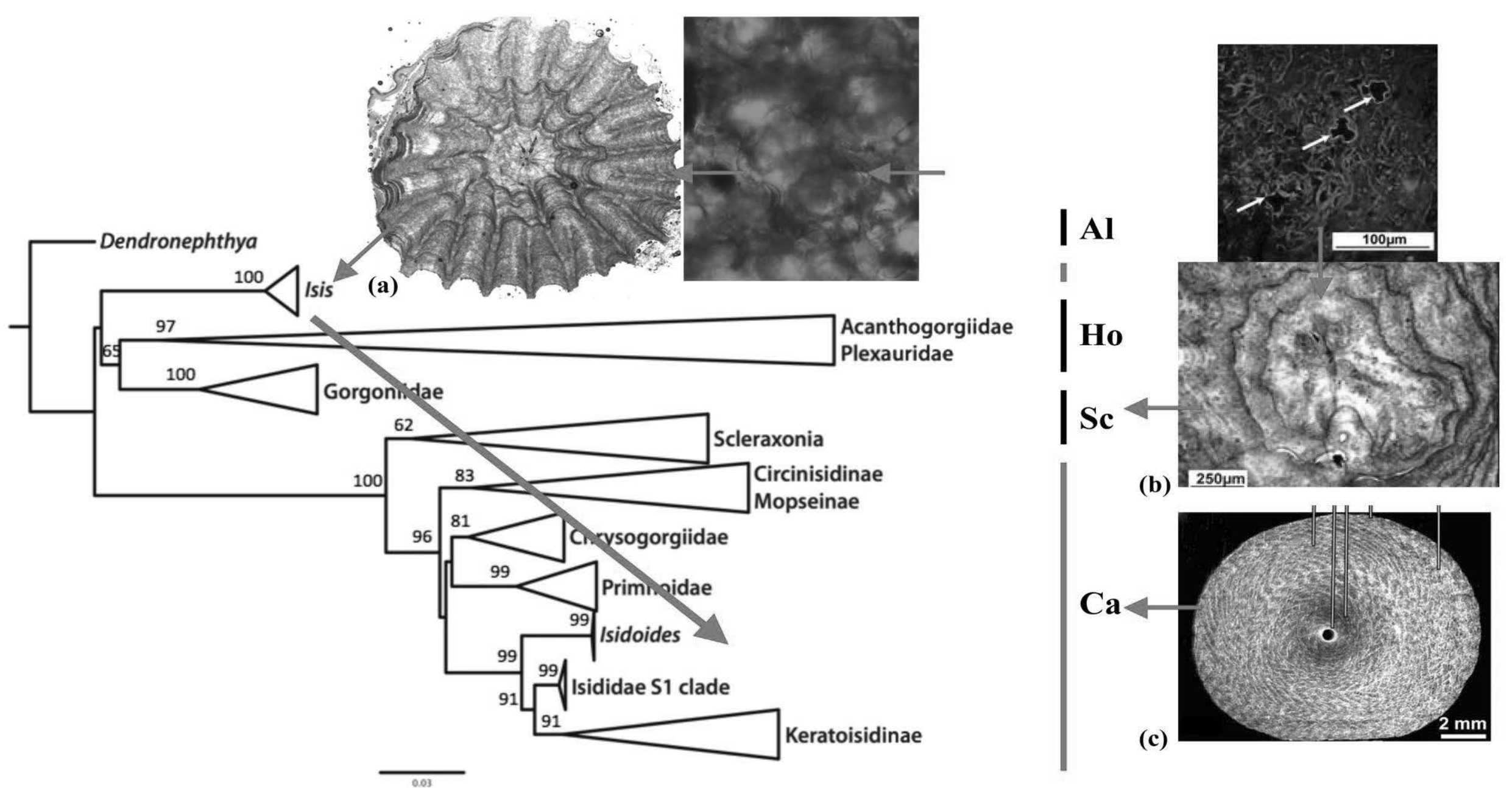

Figure 5.3. Phylogenetic reconstruction based on maximum likelihood (ML) analyses of the mt MutS (1800 bp) region (Heestand-Saucier et al. in prep.,). Axis cross-sections of (a) Isis hippuris with inset showing outline of capstan radiate composition, (b) Corallium rubrum with inset showing sclerite outline (white arrows; Debreuil et al. 2011), and (c) Keratoisis sp. (Noé \& Dullo 2006). Branch numbers represent ML support. Letters Sc $=$ Scleraxonians, Ca = Calcaxonians, Ho = Holaxonians, and Al = Alcyoniinans. Large red arrow depicts Isis separation to the rest of the Isididae (also seen in Figure 3.7). 
taxonomic groups and traits (e.g., the axis) to further investigate functional divergence or convergence within gorgonians as a consequence of environmental and/or climatic change.

Finally, it is noteworthy that I. hippuris is consistently phylogenetically situated next or close to members of the Alcyoniina (Figure 3.7, 5.3), a prominent soft coral group. Suggestions of the evolution of a central axis from the soft corals may be discarded, however, through the fossil record revealing primitive gorgonians possessing a solid axis that becomes jointed coinciding with habitat change (Helm \& Schülke 2003). Water energy is therefore considered the most selective agent for gorgonian corals (Langer 1989), further substantiating the notion of mesophotic refugia distributing bathymetric migrators which themselves evolve through local adaptation into habitat specialists.

Evidently there is a need for phylogenetic reconstruction exploiting recent advances in next generation sequencing and bioinformatics coupled with radiometric dating of fossil skeletal material (Abbey et al. 2013, Nelson et al. 2013). Targeting gorgonian taxa across specific bathymetric ranges, will inevitably shed light on both phylogenetic and evolutionary processes within the Octocorallia.

\subsection{MECHANISMS OF ECOLOGICAL DIVERGENCE}

Fitness enhancement through plasticity capacity produces phenotypic novelty in response to environmental change, particularly in modular marine organisms, ultimately leading to enhanced biodiversity over time (Levene 1953, Blanquart et al. 2013). Here, in order to test the theoretical framework of local adaptation leading to divergent selection through the action of environmental perturbation, a suite of phenotypic traits were considered and parsed into phenotypic modules (Figure 4.1; Chapter 4). Results suggested inherent plasticity capacity as evident in healthy reef morphotypes, yet through the continual action of anthropogenic disturbance on a semi-lagoonal reef, local adaptation has led to incipient divergent selection in I. hippuris within the WMNP. This can be depicted through a 'sliding scale' of phenotypic evolution between I. hippuris morphotypes in response to environmental change (Figure 5.4). The biological success of $I$. hippuris is therefore likely due to its pliable modular nature combined with a dynamic symbiotic association maintaining functional optima. Thus, further reinforcement through low reproductive dispersal and asexual fragmentation with trait fixation at the population level, plasticity capacity may then lead to genetic stability in a stress-induced phenotype with genetic incompatibility in the former, but further lead to enhanced phenotypic resilience. Whether such patterns are recapitulated throughout the I. hippuris distributional range remains to be 


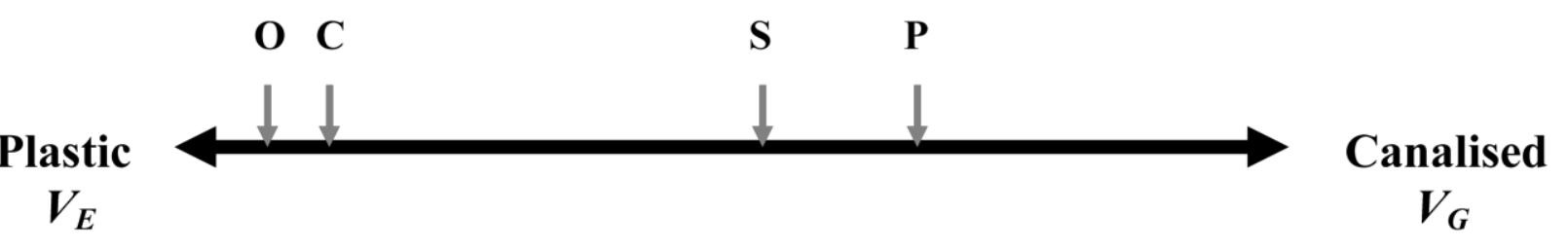

(a)

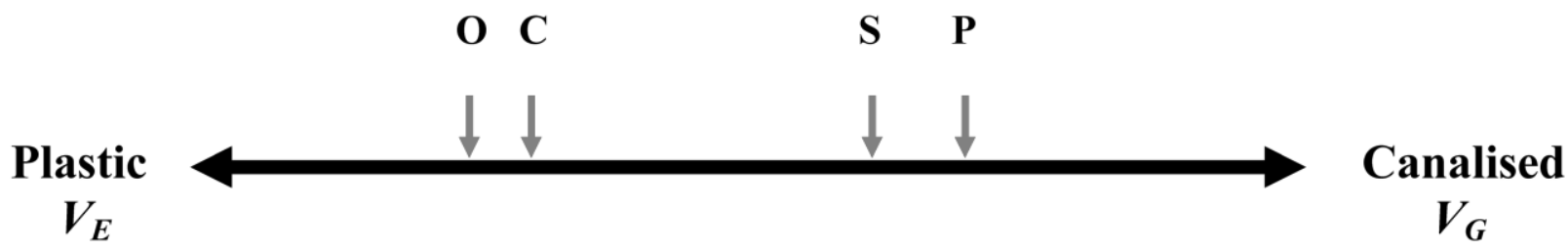

(b)

Plastic

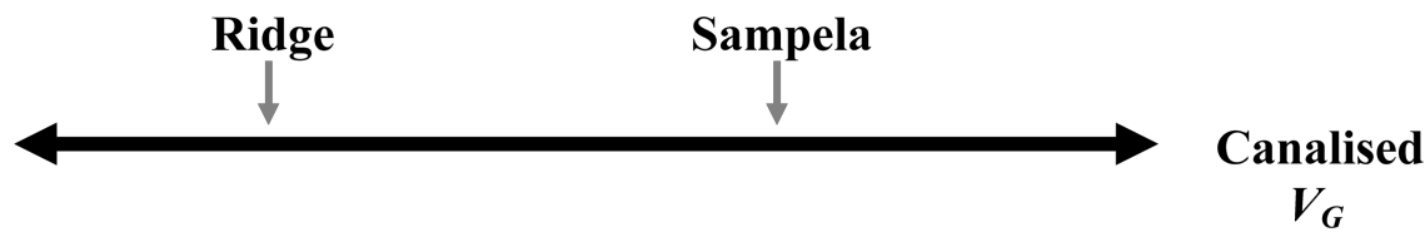

(c)

Figure 5.4. Sliding scale of phenotypic evolution within and between Isis hippuris morphotypes across habitat gradients within the WMNP, Indonesia. (a) Ridge (healthy site) morphotype responses to environmental change; (b) Sampela (impacted site) morphotype responses to environmental change, and (c) summary evolutionary trait development suggested as a consequence of plasticity capacity at the Ridge and incipient divergence through genetic assimilation of beneficial traits on degraded reefs. Red arrows depict phenotypic module position along the sliding scale of phenotypic evolutionary continuum between environmentally sensitive $\left(V_{E}\right)$ to insensitive $\left(V_{G}\right)$. Codes: $\mathrm{C}$, colony; P, polyps; $\mathrm{S}$, sclerites; O, optical parameters.

elucidated. Nonetheless I. hippuris can clearly be considered as a significant indicator of reef health, with a view for two species delineation in light of tests on reproductive isolation ascertained through cross fertilisation, and subsequent coalescence (time to divergence, as seen in Asteroidea, Puritz \& Toonen 2011, Puritz et al. 2012b).

Restricted gene flow in the face of anthropogenic disturbance likely acts at the trophic level. Polyps are the primary phenotypic module (sensu stricto) for resource acquisition through the proportional variability of heterotrophy verses autotrophy. In each I. hippuris morphotype, polyp dynamics were relatively constrained compared to other traits and consistently so in Sampela 

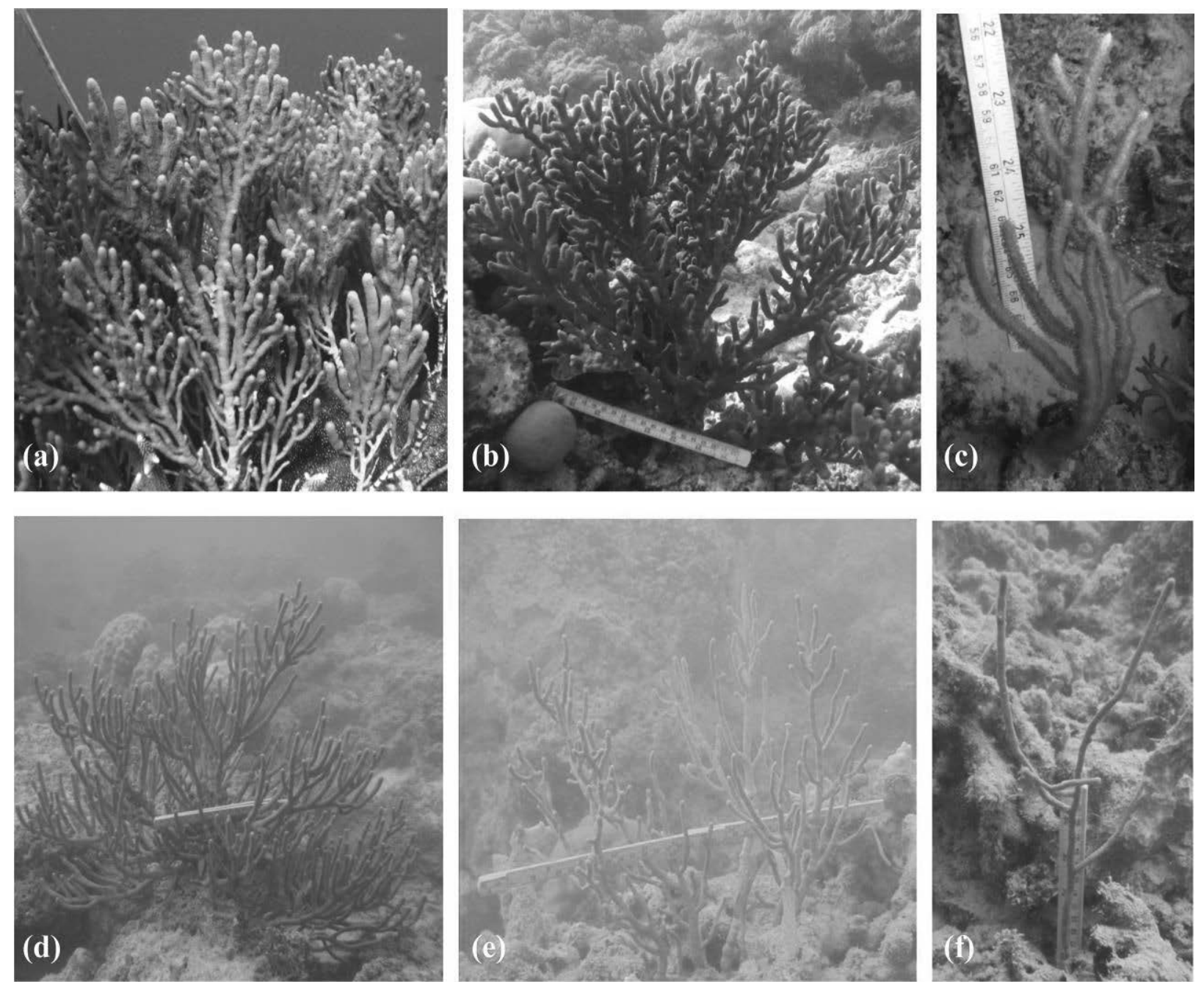

Figure 5.5. Isis hippuris morphotypes across bathymetry from the Ridge at (a) $2 \mathrm{~m}$, (b) $6 \mathrm{~m}$, (c) $14 \mathrm{~m}$. Sampela at (d) $2 \mathrm{~m}$, (e) $6 \mathrm{~m}$, (f) $10 \mathrm{~m}$.

colonies (Figure 5.4a, b). Therefore, photoacclimatory responses at the morphological and physiological level facilitate mechanistic adjustments maintaining resource acquisition by the polyp within the I. hippuris holobiont (Chapter 4). Resource, thus fitness optima in contrasting reef health environments would inevitably be reflected in photosynthetic efficiency through the non-photochemical quenching and electron transfer of PSII (Hennige et al. 2008). Moreover, phenotypic variability in native colonies across bathymetry (Figure 5.5) would therefore reveal differential photoacclimatory responses not accounted for by light-adapted yield alone, to differential environmental regimes.

Phenotypic responses across environmental gradients such as bathymetry are not unknown, particularly in zooxanthellate gorgonians (e.g., West et al. 1993, Kim et al. 2004, Prada et al. 2008, Prada \& Hellberg 2013; Figure 5.5). In figure 5.5, I. hippuris colonies exhibit clear morphological plasticity with decreased light availability. However, this response may be due to 
a differential photoacclimatory capacity between sites, which may further reinforce ecological divergent mechanisms between morphotypes. Tests of electron transfer efficiency through photosystem reaction centres would provide a much more elaborate explanation of photosynthetic responses and efficiency, and can be achieved via in situ rapid light response curve analyses with additional considerations for the photosynthetically useable radiation (PUR; MacIntyre et al. 2002, Suggett et al. 2007, Hennige et al. 2008) available to the individual at any one time. Furthermore, even though multi-marker molecular analysis strongly suggested symbiont specificity in I. hippuris irrespective of test or sampling site (Chapter 4), Rowley et al. (2011) discovered colonies that were occasionally associated with Symbiodinium clade C on degraded reefs at depth (Figure 5.6). It was concluded in Chapter 4 that the tight association with a novel type within the putatively stress tolerant clade D Symbiodinium strongly accounted for the biological success of I. hippuris morphotypes particularly on degraded reefs. Symbiodinium D cladal-types are known for their high photoacclimatory capacity, particularly in variable irradiance and highly sedimented reefs (Toller et al. 2001a, b, Baker et al. 2004, Fabricius et al. 2004, Rowan 2004, Stat \& Gates 2011). However, association with Symbiodinium Clade C particularly at depth can also be characteristic of this clade (Rowan \& Knowlton 1995, Rowan et al. 1997, Baker 2003, Chan et al. 2009, Bongaerts et al. 2010, Lesser et al. 2010). The sparse presence detected within I. hippuris morphotypes at depth may be either a consequence of developmental constraints or limited analytical detection methods. The mode of endosymbiont transmission is unknown for I. hippuris, however the additional association with a C cladal-type may be due to non-selective horizontal Symbiodinium acquisition by juvenile colonies leading to host selectivity as an adaptive environmentally-induced response. Tests of symbiont transmission and selection mechanisms, as well as fine scale Symbiodinium diversity using high resolution real-time PCR, would determine the presence of cryptic communities within individual colonies across bathymetry and reef health (Silverstein et al. 2012).

The relative contribution of light harvesting efficiency through photoacclimatory responses between I. hippuris morphotypes likely contributes to the residual error $\left(V_{\text {error }}\right)$ between phenotypic traits (Chapter 4). Additional inherent contributors such as trophic interactions within and between holobiont morphotypes will inevitably lead to differential resource allocation patterns in contrasting reef environments. Therefore, shifts in resource reliance (e.g., phototrophy versus heterotrophy) relative to the environment would result in different 


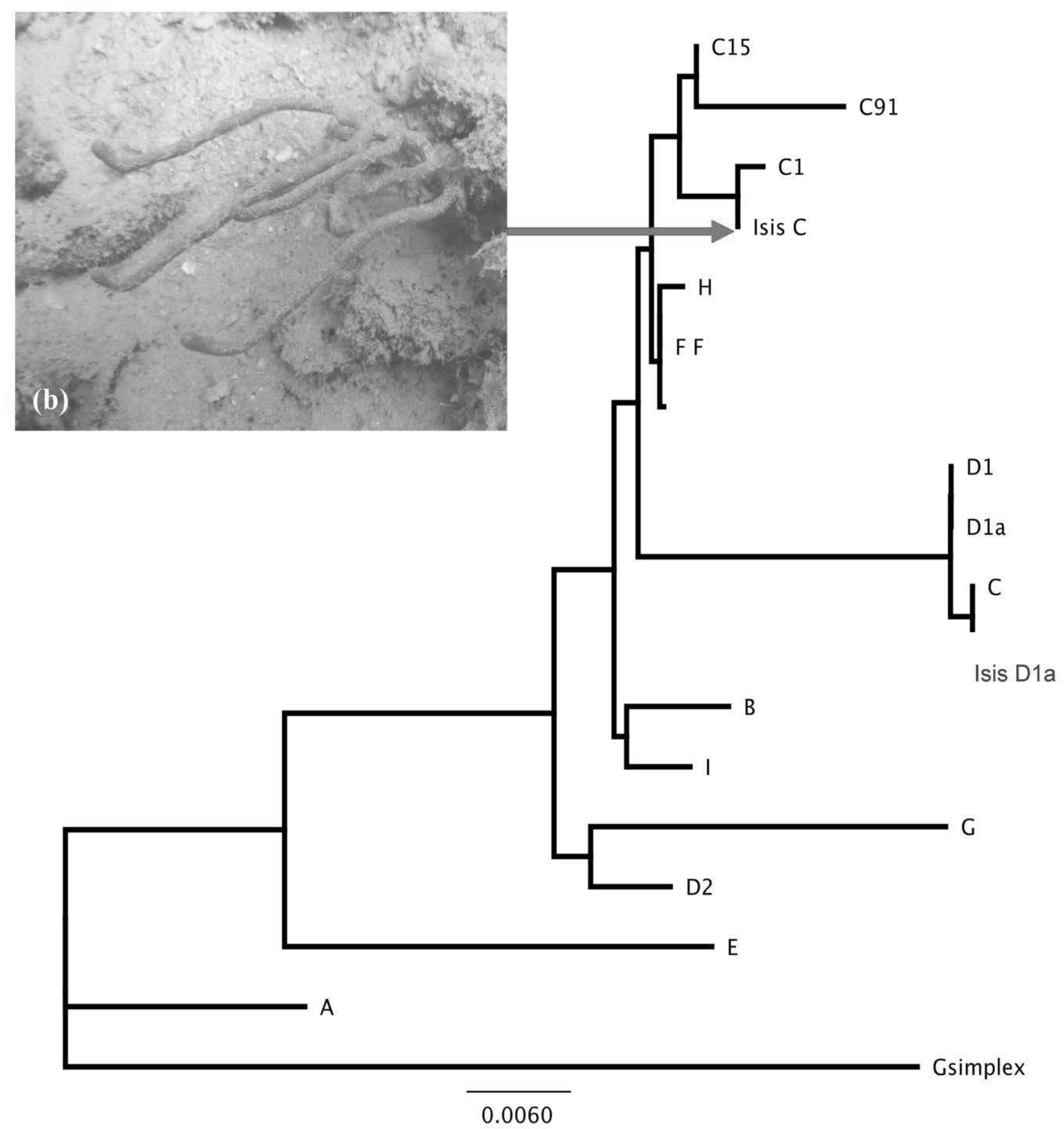

(a)

Figure 5.6. (a) Phylogenetic reconstruction of Symbiodinium clades within Isis hippuris at source depth based on maximum likelihood (ML) and bayesian inference (BI) analyses (1000 bootstrap) of the mitochondrial-encoded cytochrome oxidase COX1 region (Takabayashi et al. 2004), rooted with Gymnodinium simplex (Lohmann) Kofoid \& Swezy 1921. (b) Inset image of Isis hippuris at $12 \mathrm{~m}$ depth at Sampela containing clade C. 
physiological optima for each I. hippuris morphotype, essentially working to maximise fitness in their respective environments. Here, morphological and physiological photoacclimatory responses confirm mechanistic adjustments that maintain such fitness optima between $I$. hippuris morphotypes on contrasting reefs. Assessment of trophic sources, allocation structure, and thus extrinsic and intrinsic energy transfer role(s) within and between holobiont morphotypes would inevitably provide insight into differential benthic-pelagic coupling patterns particularly on degraded reefs. Thus, mechanisms of environmentally mediated phenotypic changes in I. hippuris at the trophic level can be tested through; 1) coral-microbiome metabolic reciprocity, and 2) comparative energy apportionment between the host soft tissue, endosymbiont and environment via carbon $\left(\delta^{13} \mathrm{C}\right)$ and nitrogen $\left(\delta^{15} \mathrm{~N}\right)$ isotopic analyses.

Firstly, alternative adaptive mechanisms may exist within the coral holobiont as a consequence of functional (e.g., nutrient) reciprocal metabolic pathways between the host and its microbiome (associate microbial ecological community). Tests of coral holobiont reciprocity through metabolic interactions using next generation sequencing (NGS) approaches, would further elucidate nutrient cycling between contrasting environments. Therefore, the characterisation of microbial community dynamics (e.g., species-specificity versus functional guilds), functional gene families and subsequent metabolic profiling through the mapping of gene expression data onto specific metabolic pathways (e.g., Larsen et al. 2011), would provide invaluable insights into integral metabolic links within the coral microbiome essentially predicted to structure holobiont trophic adaptations between environments.

Secondly, investigations into resource allocation structure within the holobiont and its environment using carbon $\left(\delta^{13} \mathrm{C}\right)$ and nitrogen $\left(\delta^{15} \mathrm{~N}\right)$ isotopic analyses, would determine if a trophic shift has occurred in response to anthropogenic disturbance, and thus, provide further corroborative evidence for environmentally mediated phenotypic responses to anthropogenic impacts. By removal of the $\mathrm{CaCO}_{3}$ skeletal elements, an accurate assessment of the carbon $\left(\delta^{13} \mathrm{C}\right)$ and nitrogen $\left(\delta^{15} \mathrm{~N}\right)$ isotopic signatures in both $I$. hippuris soft tissue and algal endosymbionts has been obtained (Appendix I). Preliminary isotope results (Figure 5.7) revealed increased carbon and nitrogen levels in the degraded site morphotype and its endosymbionts compared to that of the healthy reef. What's more, comparative signatures clearly demonstrate that both host and endosymbionts at Sampela primarily obtain heterotrophic nutrients from fish sources contained in sinking particulates (POM). With a burgeoning human population of $\sim 1600$ continuously releasing waste matter onto the reef, such corroborative isotopic values are likely sourced from human fish consumption, a heavily relied upon resource 


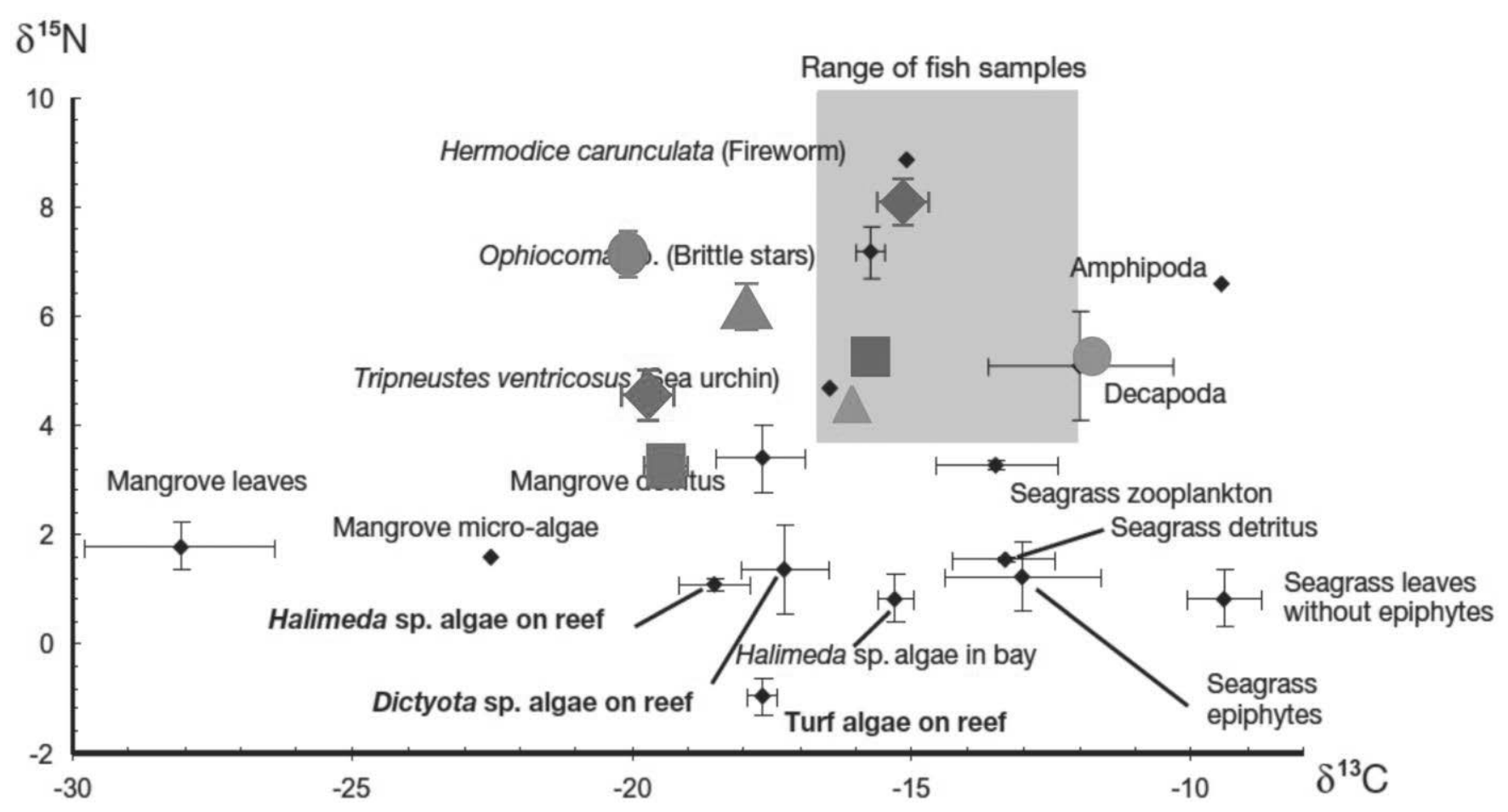

Figure 5.7. Isis hippuris holobiont $(\mathrm{n}=8)$ and environmental $(\mathrm{n}=72)$ stable isotope results (mean $\pm \mathrm{SE}$ ) from the Ridge and Sampela for carbon $\left(\delta^{13} \mathrm{C}\right)$ and nitrogen $\left(\delta^{15} \mathrm{~N}\right)$. Data are superimposed on to coral reef (in bold), sea grass and mangrove (normal font) food source values with the shaded area taken from fish muscle tissue (Figure 2; Cocheret de la Morinière et al.

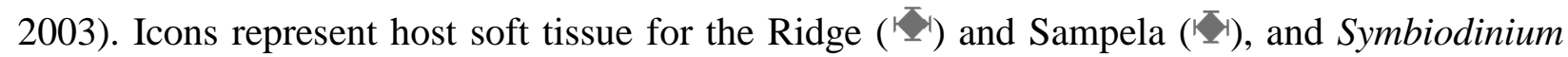

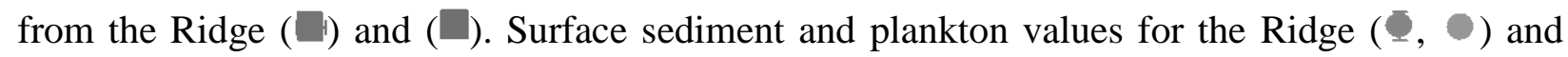
Sampela $(\bar{\Lambda}, \Delta)$ respectively.

within the community (Clifton 2013). Nevertheless, values for the Ridge (healthy site) suggest a greater reliance on phototrophy compared to allochthonous sources (Figure 5.7). It seems unlikely that light may have a significant effect on the fractionation of nitrogen as seen in the Caribbean zooxanthellate gorgonians Gorgonia ventalina Linnaeus 1758 and Pseudopterogorgia americana Gmelin 1791 between healthy and polluted reefs (Baker et al. 2011). Firstly, I. hippuris samples were taken at optical equivalent depths at the two sites and secondly, holobiont (host and Symbiodinium) isotope values were both higher and more akin to

fish levels, again suggesting alternative and greater resource supply at the degraded reef. The effect of light utilisation and trophic shifts between morphotypes could be determined through comparative $\delta^{13} \mathrm{C}$ and $\delta^{15} \mathrm{~N}$ values coupled with photosynthetic efficiency through measuring the electron transfer efficiency of PSII via in situ rapid light response curves between reciprocal transplants. Henceforth, resource allocation structure will likely shift leading to compensatory 
morpho-optical phenotypic responses as seen in Chapter 4. To fully investigate mechanisms of divergence at the trophic level, a thorough analysis between transplanted morphotypes (e.g., those in Chapter 4) would further elucidate resource allocation shifts and thus the role of phototrophy versus heterotrophy in maintaining nutritional intake under different environmental regimes.

\subsection{CONSERVATION IMPLICATIONS}

From the evidence presented here and throughout this research it is clear that human encroachment exacerbates or accelerates evolutionary processes within the marine environment, specifically gorgonian octocorals (Chapters $2-5$ ). Undoubtedly the issue at hand is at a social and economic level, and yet the situation within the WMNP appears complex (Pilgrim et al. 2007a, b, Webber 2008, Clifton et al. 2010, Clifton 2013), with continuous reproaches manifesting social and economic repercussions that are counterproductive (Clifton 2003). Traditional folklore strategies particularly in the Bajo (sea gypsy) communities (e.g., Sampela) are increasingly shifting from subsistence to income fisheries through economic development (Pilgrim et al. 2007a). Such income fisheries discard folklore and engage in unsustainable and destructive fishing practices such as cyanide, dynamite (Pilgrim et al. 2007b) and fish fences (Exton 2010). In fact the prolific use of fish fences with a 50\% decrease in mesh size has inevitably led to a marked decrease in coral reef fish abundance within the region (Exton 2010). With a concomitant increase in human population size, resource reliance and economic development, local marine resources seldom replenish with algal and/or sediment-dominated reefs, the latter increasingly the case at Sampela (see Figure 4.2b). The disturbing reality of human encroachment is acknowledged by government agencies and local communities alike, however enforcement is often favoured over community education (Clifton 2003). Well meaning in part, remedial fisheries management strategies are often implemented by conservation agencies, yet no-take zone (Unsworth et al. 2007) payoff strategies are withdrawn instilling false hope and a lack of trust in cross-cultural cooperation. It is clear that simple beneficial strategies at the local scale are productive with fisheries stock depletion ameliorated through cooperative long-term management schemes and education. Through fostering trust and cooperation with local communities in Madagascar for example, Oleson (2008) and BarnesMauthe et al. (2013) demonstrated that regular temporary octopus fishery closures and local community involvement both at the fisheries monitoring and education levels, led to significant increases in catch (Oliver et al. in prep.) and local income (Barnes-Mauthe et al. 2013, Oleson et al. in prep.). One can only hope that work such as this may be of some benefit to the local WMNP communities, yet in the face of human necessity and dogmatic perception it is hard to 
predict and sadly out of the scope of this research. Nonetheless, gorgonian octocorals are clear indicators of reef health (Chapter 2) and biological resilience, particularly in the case of $I$. hippuris - with local adaptation at the phenotypic level likely leading to ecological divergence in sympatry (Chapters $3 \& 4)$.

Conservation measures as a consequence of this work would undoubtedly benefit both coral reef and local village inhabitants alike. Ongoing ecological monitoring on an annual or six monthly basis, using belt transects as outlined in Chapter 2, would give an accurate assessment of gorgonian abundance and diversity across anthropogenic and bathymetric clines. Ecological assessments with concomitant abiotic parameter measurements would help identify areas of conservation concern for protection. Identifying the presence and abundance of the alternative yet conspicuous phenotype of I. hippuris - long-branched bushy colonies - inhabiting degraded reefs would assist in reef health assessments. Moreover, such ecological assessments coupled with trophic monitoring using stable isotope analyses of the I. hippuris holobiont and its environment (Figure 5.5 and Appendix I), would further provide a concise record of anthropogenic population expansion effects on coral reef habitats within the Wakatobi. Given the wide distribution of Isis morphotypes throughout the Indo-Pacific it is not unreasonable to propose this genus be specifically added to conservation agency annual survey lists and considered for CITES protection. This last point may seem a little severe, however, Isis hippuris (sensu lato) is still widely collected for the curios and jewellery industry (Cooper et al. 2011, Rowley pers obs.). Therefore, its presence is threatened by human encroachment and worthy of widespread monitoring awareness and protection.

Azooxanthellate gorgonian corals such as those in the deep-ocean, Mediterranean and temperate waters (e.g., Eunicella verrucosa Pallas 1766, Corallium spp. Cuvier 1798) are CITES protected and/or classified under 'vulnerable marine ecosystems' (VMEs). Deep-ocean gorgonians are a principle taxonomic group designation by the United Nations General Assembly (UNGA), safeguarded under the VME umbrella for protection against fishing activities throughout the world oceans (Rogers \& Gianni 2010, Watling et al. 2011). Yet when it comes to the tropics, this highly diverse and abundant taxonomic group receives little, if any, conservation concern. This thesis highlights two clear patterns when considering azooxanthellate gorgonians on coral reefs within the WMNP (Chapter 2) and the tropical Indo-Pacific (Figure 5.1); firstly that azooxanthellate gorgonians increase in diversity and abundance with increasing depth, and yet secondly, are sensitive to anthropogenic disturbance. These are two key points of biodiversity conservation concern, ecological response patterns not uncommon to most reef taxa. However, 
what sets gorgonians apart from other, in particular, benthic invertebrate groups, is their ubiquity and diversity throughout Indo-Pacific shallow and twilight reefs, most of which as yet undescribed (Bayer 1981). Gorgonians are also host to the CITES protected Hippocampus spp., as well as numerous species being actively exploited for commercial endeavours (e.g., Grigg 2010, Cooper et al. 2011; $21 \mathrm{~kg}$ of gorgonian and black coral were impounded by the Philippine Bureau of Fisheries and Aquatic Resources (BFAR) in 2011 for the international jewellery trade (Labe L, pers. comm., 2013). Hence, gorgonian corals are a viable conservation priority group throughout the Indo-Pacific and tropics per se, extending and/or initiating monitoring survey initiatives to the family level assessment at the very least.

With a geological age greater than Scleractinia, coupled with increased abundance and diversity on deep reefs, it is not unreasonable to propose the success of gorgonian communities over time being due to shallow reef re-population from deeper communities in the face of global climate change. Furthermore, with gorgonian skeletal material consisting of magnesium calcite (Bayer 1973), and therefore a saturation state far less sensitive to ocean acidification than scleractinia (McCulloch et al. 2012), gorgonian corals are a prominent taxonomic group within the marine realm, acting as surrogates for local and global environmental change. So even though as humans we are unable to save habitats through their ongoing destruction, mesophotic reefs of refugia likely replenish many of their inhabitants.

\subsection{CONCLUDING REMARKS}

This work summarises the importance of gorgonian sea-fan corals on reefs within the WMNP, Indonesia (and the Coral Triangle per se); an area of huge biodiversity increasingly subject to anthropogenically-induced decline. Ongoing taxonomic investigations will undoubtedly reveal new species, evolutionary processes by which they emerged, and their relative diversity over geological time enabling predicted phenotypic, and inherent population responses to environmental change. Yet these animals, and the reefs in which they reside are on borrowed time, unless the validity of the hypothesised mesophotic reef refugia holds true. Considerable polyphyly across bathymetry within groups hold valuable insights into evolutionary mechanisms over geological time as a consequence of convergence through ecological necessity, or deep divergence. Studies on ubiquitous taxa such as the zooxanthellate isidid I. hippuris provide valuable insight into reef health, in addition to the processes by which ongoing environmental perturbations lead to ecological divergence on degraded reefs. For example, the biological success of I. hippuris within the WMNP, coupled with a strong association with the putatively stress tolerant Symbiodinium clade D, makes the case for environmentally robust gorgonian 
species. However, the detection of Symbiodinium clade C at depth on degraded reefs invites tests of greater sensitivity in endosymbiont communities further reinforcing host selection control of the holobiont, and ultimately environmentally-mediated phenotypic responses to anthropogenic impacts on coral reefs. Moreover, the residual error $\left(V_{\text {error }}\right)$ between phenotypic traits (Chapter 4) further leads to tests of divergence through metagenomic community analyses and isotopic assessment in order to elucidate mechanisms of divergence at the trophic level. Ultimately, however - and from a historical perspective - it is important to discover how traits evolved to their current utility, adaptive or exaptive, therefore as the original adaptation or the by-product (Gould 1997), which often eventually facilitates the action of the whole organism. Therefore, in order to study biodiversity in its truest form, it is necessary to know what a species is, its historical origin (Rohde 1999), evolution and hence how and why individuals and populations may respond to environmental perturbations and change. A lofty task, yet awareness brings about accurate interpretation of the quintessential biological system presented before us. Thus, investigations to further understand patterns of gorgonian ecology and biology through crossdisciplinary approaches are increasingly important in management and remedial conservation efforts. 


\section{APPENDIX I: METHODS FOR STABLE ISOTOPE ANALYSES}

\section{A:1 Host and Symbiodinium Separation}

To determine the acclimatory capacity of the zooxanthellate gorgonian Isis hippuris Linnaeus 1758 between two contrasting environments, a novel analysis was successfully adapted from the hard coral literature, separating host soft tissue, algal endosymbionts and $\mathrm{CaCO}_{3}$ skeletal elements (sclerites). Host soft tissue and algal endosymbionts $(n=8)$ were first separated through three cycles of centrifugation (5 min at $6000 \mathrm{~g}$ ), vortexing (30 s), and resuspension in $1.5 \mathrm{ml}$ FSW (0.2 $\mu \mathrm{m}$ filtered sea water). The subsequent supernatant (host slurry) was filtered through replicate $0.7 \mu \mathrm{m}$ Whatman ${ }^{\circledR} \mathrm{GF} / \mathrm{F}$ (glass fiber filters, muffled at $550^{\circ} \mathrm{C}$ for $3 \mathrm{~h}$ ). The remaining $\mathrm{CaCO}_{3} /$ endosymbiont pellet was resuspended in $1 \mathrm{ml}$ Milli-Q ${ }^{\circledR}$ deionised water (MQDI) with three wash cycles (as above). Residual zooxanthellae were then retained through filtration (as above). Replicate filters (GF/F x 6 per sample including host and endosymbiont acid and non-acidified filters, as well as ash free dry weight heated at $550^{\circ} \mathrm{C}$ for $6 \mathrm{~h}$ ) for each sample colony were dried $\left(56^{\circ} \mathrm{C}\right.$ for $\left.48 \mathrm{~h}\right)$ with $3 \mathrm{~N}$ HCL addition to one replicate set - two filters, host and endosymbiont - for comparative analyses ensuring $\mathrm{CaCO}_{3}$ removal and accuracy. Replicate sample filters were folded into pre-acetone soaked 9 x $10 \mathrm{~mm}$ tin capsules $\left(\right.$ Costech Analytical ${ }^{\circledR}$ ) for downstream $\delta^{13}$ Carbon and $\delta^{15}$ Nitrogen analyses at the University of Hawai'i at Mãnoa (see below).

\section{A:2 SEDIMENT}

Environmental $\delta^{13} \mathrm{C}$ and $\delta^{15} \mathrm{~N}$ signature comparisons were conducted at both sample sites (Ridge and Sampela) in 2010 and 2011. Surficial sediment was sampled $(n=24)$ from optical equivalent depths adjacent to transplant blocks (see Figure 4.3, Chapter 4). Sediment was dried on site at $60^{\circ}$ for $48 \mathrm{~h}$ and transported in muffled $\left(550^{\circ} \mathrm{C}\right.$ for $\left.3 \mathrm{~h}\right)$ tin foil to the University of Hawai'i at Mãnoa for further processing and analyses. Samples were ground with a marble mortar and pestle, and sieved $(<125 \mu \mathrm{m})$. All instruments were sequentially cleaned prior to each sample with MQ-DI, dichloromethane, methanol and acetone to minimise crosscontamination. Untreated ground samples were weighed into $5 \times 9 \mathrm{~mm}$ tin capsules for $\delta^{15} \mathrm{~N}$ analysis. For $\delta^{13} \mathrm{C}$ analysis, replicate ground sediment samples were acidified through the aqueous/rinse-acidification method being the most effective for high carbonate $(>70 \%)$ sediment (see Komada et al. 2008, Briggs 2011, Briggs et al. in press). Hydrochloric acid (3N HCL) was slowly added to ground sediment samples in pre-weighed $50 \mathrm{~mL}$ centrifuge tubes until no further effervescence was detected. Acid-treated sediments underwent 3 x $10 \mathrm{~mL}$ MQ-DI, vortex, and centrifuge ( $5 \mathrm{~min}$ at $3000 \mathrm{~g}$ ) wash cycles, and subsequently dried $\mathrm{O} / \mathrm{N}$ at $60^{\circ} \mathrm{C}$. Dried 
samples were re-weighed into 5 x $9 \mathrm{~mm}$ silver capsules for $\delta^{13} \mathrm{C}$ analysis. Note: results were similar within sites (i.e., depth) and therefore pooled and presented in Figure 5.7 (Chapter 5).

\section{A:3 PLANKTON}

Water column $\delta^{13} \mathrm{C}$ and $\delta^{15} \mathrm{~N}$ signature comparisons were conducted using vertical and horizontal plankton tows between 00:00 - 01:00 $\mathrm{h}$ and 07:00 - 08:00 $\mathrm{h}$ at both sample sites (Ridge and Sampela) in 2010 and 2011. Plankton samples $(n=48)$ were dried on site at $60^{\circ} \mathrm{C}$ for $48 \mathrm{~h}$ and transported in muffled tin foil for downstream processing and analyses. Dried samples were ground and directly weighed into $5 \times 9$ tin capsules for $\delta^{15} \mathrm{~N}$, and silver capsules for $\delta^{13} \mathrm{C}$ via acidification. Acidified ( 3 drops of $3 \mathrm{~N}$ HCL or until effervescence ceased) replicates were dried $\mathrm{O} / \mathrm{N} 60^{\circ} \mathrm{C}$ and re-weighed prior to $\delta^{13} \mathrm{C}$ analysis. Note: results were indeterminate within sites (i.e., vertical and horizontal) and so pooled and presented in Figure 5.7 (Chapter 5).

All $\delta^{13}$ Carbon and $\delta^{15}$ Nitrogen were determined using a Carlo Erba NA 2500 elemental analyser, interfaced via a ConFlo II to a Delta Plus mass spectrometer (Finnigan, Inc), at the University of Hawai'i at Mãnoa, USA. 


\section{REFERENCES}

Abbey E, Webster JM, Braga JC, Jacobsen GE, Thorogood G, Thomas AL, Camoin G, Reimer PJ, Potts DC. (2013) Deglacial mesophotic reef demise on the Great Barrier Reef. Palaeogeogr. Palaeoclimatol. Palaeoecol. 392: 473-494

Abràmoff M, Magalhaes PJ, Ram SJ. (2004) Image processing with ImageJ. Bioph. Int. 11: 36-42

Abrego D, Ulstruo KE, Willis BL, van Oppen MJH. (2008) Species-specific interactions between algal endosymbionts and coral hosts define their bleaching response to heat and light stress. Proc. R. Soc. Lond. B. 275: 2273-2282

Aguilar C, Sánchez JA. (2007a) Phylogenetic hypotheses of gorgoniid octocorals according to ITS2 and their predicted RNA secondary structures. Mol. Phylogenet. Evol. 43(3): 774-786

Aguilar C, Sánchez JA. (2007b) Molecular morphometrics: contribution of ITS2 sequences and predicted RNA secondary structures to octocoral systematics. Bull. Mar. Sci. 81(3): 335-349

Akaike H. (1974) New look at statistical model identification. IEEE Trans. Autom. Contr. 19: 716-723

Alderslade P. (1998) Revisionary systematics in the gorgonian family Isididae, with descriptions of numerous taxa (Coelenterata: Octocorallia). Records of the Western Australian Museum, Supplement No. 55: 1-359

Anderson MJ. (2001) A new method for non-parametric multivariate analysis of variance. Aus. Ecol. 26: $32-46$

Anderson MJ. (2003) DISTLMforward: a FORTRAN computer program to calculate a distance based multivariate analysis for a linear model using forward selection. Department of Statistics, University of Auckland, New Zealand.

Anderson MJ, Willis TJ. (2003) Canonical analysis of principal coordinates: a useful method of constrained ordination for ecology. Ecology. 84: 511-524

Anthony KRN. (2000) Enhanced particle-feeding capacity of corals on turbid reefs (Great Barrier Reef, Australia). Coral Reefs. 19: 59-67

Anthony KRN, Fabricius KE. (2000) Shifting roles of heterotrophy and autotrophy in coral energetics under varying turbidity. J. Exp. Mar. Biol. Ecol. 252: 221-253 
Apprill A, Gates RD. (2007) Recognizing diversity in coral symbiotic dinoflagellate communities. Mol. Ecol. 16:1127-1134

Apprill A, Marlow HQ, Martindale MQ, Rappé MS. (2009) The onset of microbial associations in the coral Pocillopora meandrina. ISME. 1-15

Aronson RB, Precht WF. (1995) Landscape patterns of reef coral diversity: A test of the intermediate disturbance hypothesis. J. Exp. Mar. Biol. Ecol. 192: 1-14

Aubin-Horth N, Renn SCP. (2009) Genomic reaction norms: using integrative biology to understand molecular mechanisms of phenotypic plasticity. Mol. Ecol. 18: 3763-3780. doi: 10.1111/j.1365294X.2009.04313.x

Aurivillius M. (1931) The Gorgonarians from Dr. Sixten Bock's expedition to Japan and Bonin Islands 1914. Kungliga Svenska Vetenskapsakademiens Handlingar. 3(9) 4: 1-337

Baker AC. (2003) Flexibility and specificity in coral-algal symbiosis: diversity, ecology, and biogeography of Symbiodinium. Annu. Rev. Ecol. Evol. Syst. 34: 661-689

Baker AC, Romanski AM. (2007) Multiple symbiotic partnerships are common in scleractinian corals, but not in octocorals: Comment on Goulet (2006). Mar. Ecol. Prog. Ser. 335: 237-242

Baker AC, Starger CJ, McClanahan TR, Glynn PW. (2004) Coral reefs: corals' adaptive response to climate change. Nature. 430: 741

Baker DM, Kim K, Andras JP, Sparks JP. (2011) Light-mediated ${ }^{15} \mathrm{~N}$ fractionation in Caribbean gorgonian octocorals: implications for pollution monitoring. Coral Reefs. 30: 709-717. DOI 10.1007/s00338-011-0759-x

Barnes-Mauthe M, Oleson KLL, Zafindrasilivonona B. (2013) The total economic value of small-scale fisheries with a characterization of post-landing trends: An application in Madagascar with global relevance. Fish. Res. 147: 175-185

Barshis D. (2009) Plastic versus fixed influences on coral response to fluctuating environmental conditions: sources of coral resilience and stress tolerance in a changing climate. PhD Dissertation. University of Hawai'i at Mãnoa, USA.

Bayer FM. (1955) Contributions to the nomenclature, systematics, and morphology of the Octocorallia. Proc. U.S. Nat. Mus. 105(3357): 207-220, pl.8 
Bayer FM. (1955) Descriptions and redescriptions of the Hawaiian Octocorals collected by the U.S. fish commission steamer "Albatross" (2. Gorgonacea: Scleraxonia). Pac. Sci. 10(1): 67-95

Bayer FM 1956. Octocorallia. In: Moore RC (ed) Treatise on invertebrate paleontology, Part F, Coelenterata. Geological Society of America, Lawrence, KS, p F166-190, 192-231, figs 134-162

Bayer FM. (1961) The shallow-water Octocorallia of the West Indian region. [viii] + 1-373, 101 figs, frontisp., 28 pls. The Hague: Martinus Nijhoff.

Bayer FM. (1981) Key to the genera of Octocorallia exclusive of Pennatulacea (Coelenterata: Anthozoa), with diagnoses of new taxa. Proc. Biol. Soc. Wash. 94: 901-947

Bayer FM, Grasshoff M, Verseveldt I eds. (1983) Illustrated trilingual glossary of morphological and anatomical terms applied to Octocorallia. E. 1. Brill/Dr. W. Backhuys, Leiden. pp.75, 20 pls.

Bayer FM, Stefani J (1987) Isididae (Gorgonacea) de Nouvelle-Calédonie--Nouvelle clé des genres de la famille. Bull. Mus. Natn. Hist. Nat. Paris, (4 sér.) 9 (section A) No. 1:47-106, pls. 1-30

Bayer FM, Cairns SD. (2004) The Alcyonaria of The "Blake" Expeditions. With revised explanations of the figures transcribed from A.E. Verrill's original typescript. The Unplublished Plates. Nat. Mus. Nat. Hist. Wash. pl. 102

Bayer MM, Cormack RM, Todd CD. (1994) Influence of food concentration on polypide regression in the marine bryozoan Electra pilosa (L.) (Bryozoa: Cheilostomata). J. Exp. Mar. Biol. Ecol. 178: 3550

Beiring EA, Lasker HR. (2000) Egg production by colonies of a gorgonian coral. Mar. Ecol. Prog. Ser. 196: $169-177$

Bell JJ, Barnes DK. (2000) A sponge diversity centre within a marine island. Hydrobiologia. 440: 55-64

Bell JJ, Smith DJ. (2004) Ecology of sponge assemblages (Porifera) in the Wakatobi region, south-east Sulawesi, Indonesia: richness and abundance. J. Mar. Biol. Ass. UK. 84: 581-589

Bellantuono AJ, Hoegh-Guldberg O, Rodriguez-Lanetty M. (2012) Resistance to thermal stress in corals without changes in symbiont composition. Proc. R. Soc. B. 279: 1100-1107. doi:10.1098/rspb.2011.1780 
Benayahu, Y. \& Loya, Y. (1981) Competition for space among coral-reef sessile organisms at Eilat, Red Sea. Bull. Mar. Sci. 31: 514-522

Bengston S. (1981) Atractosella, a Silurian Alcyonacean Octocoral. J. Paleontol. 55(2): 281-294

Bergman A, Siegal ML. (2003) Evolutionary capacitance as a general feature of complex gene networks. Nature. 424: 549-552

Berntson EA. (1998) Evolutionary patterns within the Anthozoa (phylum Cnidaria) reflected in ribosomal gene sequences. Doctoral thesis, Massachusettes Institute of Technology/Woods Hole Oceanographic Institution Joint Program in Oceanograpy. 98-24, Woods Hole

Berntson EA, Bayer FM, McArthur, France SC. (2001) Phylogenetic relationships within the Octocorallia (Cnidaria: Anthozoa) based on nuclear 18S rRNA sequences. Mar. Biol. 138: 235-246

Bird CE, Fernandez-Silva I, Skillings DJ, Toonen RJ. (2012) Sympatric speciation in the post "Modern Synthesis" era of evolutionary biology. Evol. Biol. 39: 158-180

Birkeland C. (1974). The effect of wave action on the population dynamics of Gorgonia ventalina Linnaeus. Stud. Trop. Oceanogr 12: 115-126

Blanquart F, Kaltz O, Nuismer S, Gandon S. (2013) A practical guide to measuring local adaptation. Ecol. Lett. doi: 10.1111/ele.12150

Bloom AJ, Chapin III SF, Mooney HA. (1985) Resource limitation in plants - An economic analogy. Ann. Rev. Ecol. Syst. 16: 363-392

Bohn K, Pavlick R, Reu B, Kleidon A. (2014) The strengths of r- and K-selection shape diversitydisturbance relationships. PLOS ONE. 9(4): e95659. doi:10.1371/journal.pone.0095659

Bongaerts P, Riginos C, Ridgway T, Sampayo EM, van Oppen MJH, Englebert N, Vermeulen F, HoeghGuldberg O. (2010) Genetic divergence across habitats in the widespread coral Seriatopora hystrix and its associated Symbiodinium. PLOS ONE. 5(5): e10871. doi:10.1371/journal.pone.0010871

Bordeleau M, Mori A, Oberer M, Lindqvist L, Chard LS, Higa T, Belsham GJ, Wagner G, Tanaka J, Pelletier J. (2006). Functional characterization of IRESes by an inhibitor of the RNA helicase Eif4A. Nat. Chem. Biol. 2: 213-220 
Bowen T, Bourne D, Rodriguez-Lanetty M. (2013) Transcriptional Activation of $c 3$ and $h s p 70$ as part of the immune response of Acropora millepora to bacterial challenges. PLos ONE. 8(7): e67246

Braendle C, Flatt T. (2006) A role for genetic accommodation in evolution? BioEssays. 28: 868-873

Brazeau DA, Harvell CD. (1994) Genetic structure of local populations and divergence between growth forms in a clonal invertebrate, the Caribbean octocoral Briareum asbestinum. Mar. Biol. 119: 53-60

Brazaeu DA, Lasker HR. (1989) The Reproductive Cycle and Spawning in a Caribbean Gorgonian. Biol. Bull. 176: 1-7

Brazaeu DA, Lasker HR. (1990) Sexual reproduction and external brooding by the Caribbean gorgonian Briareum asbestinum. Mar. Biol. 104: 465-474

Bridge TCL, Fabricius KE, Bongaerts P, Wallace CC, Muir PR, Done TJ, Webster JM. (2012) Diversity of Scleractinia and Octocorallia in the mesophotic zone of the Great Barrier Reef, Australia. Coral Reefs. 31: 179-189. DOI 10.1007/s00338-011-0828-1

Briggs R. (2011) Organic matter remineralization in coastal sediments in and around Kane'ohe bay, Hawai ‘i. PhD Dissertation. University of Hawai’i at Mãnoa, USA.

Briggs R, Ruttenberg K, Ricardo A, Popp B, Gier E. (in press.) Quantifying organic carbon and nitrogen concentration and isotopic compositions in carbonate-dominated coastal marine sediments. Limnol. Oceanog. Methods.

Brown BE, Dunne RP, Warner ME, Ambarsari I, Fitt WK, Gibbs SW, Cummings DG. (2000) Damage and recovery of photosystem II during a manipulative field experiment on solar bleaching in the coral Goniastrea aspera. Mar. Ecol. Prog. Ser. 195: 117-124 (doi:10.3354/meps195117)

Brown BE, Downs CA, Dunne RP, Gibb SW. (2002) Exploring the basis of thermotolerance in the reef coral Goniastrea aspersa. Mar. Ecol. Prog. Ser. 242: 119-129

Buhl-Mortensen L, Mortensen PB. (2004) Symbiosis in deep-water corals. Symbiosis. 37: 33-61

Buhl-Mortensen L, Vanreusel A, Gooday AJ, Levin LA, Priede IG, Buhl-Mortensen P, Gheerardyn H, King NJ, Raes M. (2010) Biological structures as a source of habitat heterogeneity and biodiversity on the deep ocean margins. Mar. Ecol. 31: 21-50 
Cadena NJ, Rey C, Hernández-Hoyos M, Sánchez JD, Teillaud S, Ardila N, Sánchez JA. (2010) Linking local to global properties in branching modular networks: gorgonian coral colonies. Mar. Biol. 157: 1003-1010. doi 10.1007/s00227-009-1380-1

Calosi P, Bilton DT, Spicer JI, Votier SC, Atfield A. (2010) What determines a species' geographical range? Thermal biology and latitudinal range size relationships in European diving beetles (Coleoptera: Dytiscidae). J. Anim. Ecol. 79: 194-204

Campbell DR. (2003) Natural selection in Ipomopsis hybrid zones: implications for ecological speciation. New Phytologist. 161: 83-90

Carlon DB, Budd AF, Lippé C, Andrew RL. (2011) The quantitative genetics of incipient speciation: heritability and genetic correlations of skeletal traits in populations of diverging Favia fragum ecomorphs. Evolution. 65-12: 3428-3447

Carpenter KE, Springer VG. (2005) The center of the center of marine shore fish biodiversity: the Philippine Islands. Env. Biol. Fish. 72: 467-480

Carpenter KE, Barber PH, Crandall ED, Ablan-Lagman MCA, Ambariyanto, Mahardika GN, ManjajiMatsumoto BM, Juinio-Meñez MA, Santos MD, Starger CJ, Toha AHA. (2011) Comparative phylogeography of the coral triangle and implications for marine management. J. Mar. Biol. 2011: 114 doi: $10.1155 / 2011 / 396982$

Cerrano C, Bavestrello G, Bianchi CN. Cattaneo-vietti R, Bava S, Morganti C, Morri C, Picco P, Sara G, Schiaparelli S, Siccardi A, Sponga F. (2000) A catastrophic mass-mortality episode of gorgonians and other organisms in the Liguirian Sea (North-western Mediterranean), summer 1999. Ecol. Lett. 3: $284-293$

Cerrano C, Danovaro R, Gambi C, Pusceddu A, Riva A, Schiaparelli. (2010) Gold coral (Savalia savaglia) and gorgonian forests enhance benthic biodiversity and ecosystem functioning in the mesophotic zone. Biodivers. Conserv. 19: 153-167

Chanmethakul T, Chansang H, Watanasit S. (2010) Soft coral (Cnidaria: Alcyonacea) distribution patterns in Thai waters. Zool. Stud. 49(1): 72-84

Chen WH, Wang SK, Duh CY. (2011) Polyhydroxylated steroids from the bamboo coral Isis hippuris. Mar. Drugs. 9: 1829-1839 
Chinnusamy V, Zhu JK. (2009) Epigenetic regulation of stress responses in plants. Curr. Opin. Plant Biol. 12: 133-139. (doi:10.1016/j.pbi.2008.12.006)

Chown SL, Gaston KJ. (2000) Areas, cradles and museums: the latitudinal gradient of species richness. Trends in Ecology \& Evolution. 15: 311-315

Clarke KR. (1993) Non-parametric multivariate analyses of changes in community structure. Aust J Ecol. $18: 117-143$

Clarke KR, Green RH. (1988) Statistical design and analysis for a 'biological effects' study. Mar. Ecol. Prog. Ser. 46: 213-226

Clarke KR, Ainsworth M. (1993) A method of linking multivariate community structure to environmental variables. Mar. Ecol. Prog. Ser. 92: 205-219

Clarke KR, Gorley RN. (2006) PRIMER v6: User Manual/Tutorial (2 ${ }^{\text {nd }}$ Ed.). PRIMER-E Ltd, Plymouth, $\mathrm{UK}$

Clarke KR, Chapman MG, Somerfield PJ, Needham HR. (2006a) Dispersion-based weighting of species counts in assemblage analyses. Mar. Ecol. Prog. Ser. 320: 11-27

Clarke KR, Somerfield PJ, Chapman MG. (2006b) On resemblance measures for ecological studies, including taxonomic dissimilarities and a zero-adjusted Bray-Curtis coefficient for denuded assemblages. J. Exp. Mar. Biol. Ecol. 330: 55-80

Clavico EE, De Souza AT, Da Gama BA, Pereira RC. (2007) Antipredator defense and phenotypic plasticity of sclerites from Renilla muelleri, a tropical sea pansy. Biol. Bull. 213: 135-140

Clement M, Posada D, Crandall KA. (2000) TCS: a computer program to estimate gene genealogies. Mol. Ecol. 9: 1657-1660

Clifton J. (2003) Prospects for co-management in Indonesia's marine protected areas. Mar. Policy. 27: 389-395

Clifton J. (2013) Refocusing conservation through a cultural lens: Improving governance in the Wakatobi National Park, Indonesia. Mar. Pol. 41: 80-86 
Clifton J, Cullen LC, Haapkylä J, Unsworth RKF. (2010) Ensuring appropriate and proportionate responses to environmental threats: A response to Caras and Pasternak. Ocean Coast. Man. 53: 700702

Cocheret de la Morinière E, Pollux BJA, Nagelkerken I, Hemminga MA, Huiskes AHL, van der Velde G. (2003) Ontogenetic dietary changes of coral reef fishes in the mangrove-seagrass-reef continuum: stable isotopes and gut content analysis. Mar. Ecol. Prog. Ser. 246: 279-289

Coffroth MA, Lasker HR (1998) Population structure of a clonal gorgonian coral: the interplay between clonal reproduction and disturbance. Evolution. 52(2): 379-393

Coffroth MA, Santos SR, Goulet TL. (2001) Early ontogenetic expression of specificity in a cnidarianalgal symbiosis. Mar. Ecol. Prog. Ser. 222: 85-96

Coma R, Zabala M, Gili JM. (1995) Sexual reproductive effort in the Mediterranean gorgonian Paramuricea clavata. Mar. Ecol. Prog. Ser. 117: 185-192

Colin PL, Devaney DM, Hillis-Colinvaux L, Suchanek TH, Harrison, III JT. (1986) Geology and biological zonation of the reef slope, 50-360 m depth at Enewetak atoll, Marshall islands. Bull. Mar. Sci. 38(1): 111-128

Coma R, Ribes M, Zabala M, Gili JM. (1995) Reproduction and cycle of gonadal development in the Mediterranean gorgonian Paramuricea clavata. Mar. Ecol. Prog. Ser. 117: 173-183

Concepcion GT, Crepeau M, Wagner D, Kahng SE, Toonen RJ. (2008) An alternative to ITS, a hypervariable, singlecopy nuclear intron in corals, and its use in detecting cryptic species within the octocoral genus Carijoa. Coral Reefs. 27: 323-336

Connell JH. (1978) Diversity in tropical forest and coral reefs. Science. 199: 1302-1310

Connell JH. (1985) The consequences of variation in initial settlement vs. post-settlement mortality in rocky intertidal communities. J. Exp. Mar. Biol. 93: 11-45

Cooper EWT, Torntore SJ, Leung ASM, Shadbolt T, Dawe C. (2011) Guide to the identification of precious and semi-precious corals in commercial trade. TRAFFIC North America \& WWF-Canada. Vancouver.

Cope JCW. (2005) Octocorallian and hydroid fossils from the Lower Ordovician of Wales. Palaeontology. 48(2): 433-445 
Costantini F, Tinti F, Abbiati M. (2003) Sistematica molecolare e filogenesi di Corallium rubrum. Biol. Mar. Med. 10, 73-75

Cornell HV, Karlson RH. (2000) Coral species richness: ecological versus biogeographical influences. Coral Reefs. 19: 37-49

Cossins A, Fraser J, Hughes M, Gracey A. (2006) Post-genomic approaches to understanding the mechanisms of environmentally induced phenotypic plasticity. J. Exp. Biol. 209: 2328-2336

Costantini F, Tinti F, Abbiati M. (2003) Sistematica molecolare e filogenesi di Corallium rubrum. Biol. Mar. Med. 10: 73-75

Cowen RK, Paris CB, Srinivasan A. (2006) Scaling of connectivity in marine populations. Science. 311: $522-527$

Crabbe JC, Smith DJ. (2003) Computer modeling and estimation of recruitment patterns of nonbranching coral colonies at three sites in the Wakatobi Marine Park, S.E. Sulawesi, Indonesia; implications for coral reef conservation. Comp. Biol. Chem. 27: 17-27, doi:10.1016/S14769271(02)00091-9

Crabbe JC, Smith DJ. (2005) Sediment impacts on growth rates of Acropora and Porites corals from fringing reefs of Sulawesi, Indonesia. Coral Reefs. 24: 437-441, doi:10.1007/s00338-005-0004-6

Crossland CJ, Barnes DJ. (1974) The role of metabolic nitrogen in coral calcification. Mar. Biol. 28: 325332

Dai CF. (1990) Interspecific competition in Taiwanese corals with special reference to interactions between alcyonaceans and scleractinians. Mar. Ecol. Prog. Ser. 60: 291-297

Dahan M, Benayahu Y. (1997) Reproduction of Dendronephthya hemprichi (Cnidaria: Octocorallia): year-round spawning in an azooxanthellate soft coral. Mar. Biol. 129: 573-579

Darriba D, Taboada G, Doallo R, Posada D. (2012) jModelTest 2: more models, new heuristics and parallel computing. Nature Methods. 9(8): 772

Darty K, Denise A, Ponty Y. (2009) VARNA: Interactive drawing and editing of the RNA secondary structure. Bioinformatics. 25: 1974-1975. doi:10.1093/bioinformatics/btp250

Darwin C. (1873) The Origin of Species (Murray, London), $6^{\text {th }}$ Ed. 
Davy SK, Allemand D, Weis VM. (2012) Cell biology of Cnidarian-Dinoflagellate symbiosis. Microbiol. Mol. Biol. Rev. 76(2): 229. DOI: 10.1128/MMBR.05014-11

Dauget J-M. (1992) Effets d'un changement d'orientation de la colonie sure la morphologie de Isis hippuris Linne, 1758 (Gorgonacea): note preliminaire. Bull. Soc. Zool. Fr. 11(4): 375-382

Dayton PK. (2003) The importance of the Natural Sciences to Conservation. (An American Society of Naturalists Symposium Paper). Am. Nat. 162(1): 1-13

Debreuil J, Tambutte S, Zoccola D, Segonds N, Techer N, Marschal C, Allemand D, Kosuge S, Tambutté É. (2011) Specific organic matrix characteristics in skeletons of Corallium species. Mar. Biol. 158: 2765-2774. DOI 10.1007/s00227-011-1775-7

De Forest L, Drazen J. (2009) The influence of a Hawaiian seamount on mesopelagic micronekton. Deep-Sea Res. I. 56: 232-250

de Kroon H, Huber H, Stuefer JF, van Groenendael JM. (2005) A modular concept of phenotypic plasticity in plants. New Phytol. 166: 73-82

De Rosa R, Grenier JK, Andreeva T, Cook CE, Adoutte A, Akam M, Carroll SB, Balavoine G. (1999) Hox genes in brachiopods and priapulids and protostome evolution. Nature. 399: 772-776

DeWitt TJ, Scheiner SM. (2004) Phenotypic variation from single genotypes. In: Phenotypic Plasticity: Functional and conceptual approaches (eds. DeWitt TJ, Scheiner SM), pp. 1-9. Oxford University Press, New York

Dove S, Ortiz JO, Enriquez S, Fine M, Fisher P, Iglesias-Prieto R, Thornhill D, Hoegh-Guldberg O. (2006) Response of holosymbiont pigments from the scleractinian coral Montipora monasteriata to short-term heat stress. Limnol. Oceanogr. 51(2): 1149-1158

Dubinsky Z, Falkowski PG, Porter JW, Muscatine L. (1984) Absorption and utilization of radiant energy by light- and shade-adapted colonies of the hermatypic coral Stylophora pistillata. Proc. R. Soc. Lond. B. 222: 203-214

Dubinsky Z, Falkowski PG, Wyman K. (1986) Light harvesting and utilization in phytoplankton. Plant Cell Physiol. 27:1335-1350 
Dubinsky Z, Stambler N, Ben-Zion M, McClosky L, Falkowski PG, Muscatine L. (1990) The effects of external nutrient resources on the optical properties and photosynthetic efficiency of Stylophora pistillata. Proc. R. Soc. Lond. B. 239: 231-245

Dueñas LF, Sánchez JA. (2009) Character lability in deep-sea bamboo corals (Octocorallia, Isididae, Keratoisidinae). Mar. Ecol. Prog. Ser. 397: 11-23

Edinger EN, Kolasa J, Risk MJ. (2000) Biogeographic variation in coral species diversity on coral reefs in three regions of Indonesia. Div Dist. 6: 113-127

Eldredge N, Gould SJ. (1972) Punctuated equilibria: an alternative to phyletic gradualism. Models in paleobiology. (ed. by J. M. Schopf), pp. 82-115. Freeman, Cooper and Co., San Francisco

Ellis J, Solander D. (1786) The Natural History of many curious and uncommon Zoophytes, collected from various parts of the globe by the late John Ellis, systematically arranged and described by the late Daniel Solander. — London, i-xii + 1-208 pp., 63 pls

English SA, Wilkinson C, Baker VJ. (1997) Survey manual for tropical marine resources. Australian Institute of Marine Science (AIDAB), Townsville, Australia. pp. 390

Enríquez S, Sand-Jensen K. (2003) Variation in light absorption properties of Mentha aquatica L. as a Function of leaf form: implications for plant growth. Int. J. Plant Sci. 164(1): 125-136

Enríquez S, Méndez ER, Iglesias-Prieto R. (2005) Multiple scattering on coral skeletons enhances light absorption by symbiotic algae. Limnol. Oceanogr. 50(4): 1025-1032

Etnoyer PJ, Wirshing HH, Sánchez JA. (2010) Rapid assessment of octocoral diversity and habitat on Saba Bank, Netherlands Antilles. PLoS ONE. 5(5): doi:10.1371/journal.pone.0010668

Excoffier L, Lischer HEL. (2010) Arlequin suite ver 3.5: A new series of programs to perform population genetics analyses under Linux and Windows. Mol. Ecol. Res. 10: 564-567

Exton D. (2010) Nearshore fisheries of the Wakatobi. In: Clifton J, Unsworth RFK, Smith DJ, [Ed]. Marine research and conservation in the Coral Triangle: the Wakatobi National Park. New York: Nova Science Publishers. pp. 193-208

Fabricius KE, Klumpp DW. (1995) Widespread mixotrophy in reef-inhabiting soft corals: the influence of depth, and colony expansion and contraction on photosynthesis. Mar. Ecol. Prog. Ser. 125:195204 
Fabricius KE, Alderslade P. (2001) Soft corals and sea fans: a comprehensive guide to the tropical shallow-water general of the Central-West Pacific, the Indian Ocean and the Red Sea. AIMS (AIDAB), Townsville. pp.264

Fabricius KE, De'ath G. (2004) Identifying ecological change and its causes: a case study on coral reefs. Ecol. App. 14(5): 1448-1465

Fabricius KE, McCorry D. (2006) Changes in octocoral communities and benthic cover along a water quality gradient in the reefs of Hong Kong. Mar. Poll. Bull. 52: 22-33

Fabricius KE, Mieog JC, Colin PL, Idip D, van Oppen MJH. (2004) Identity and diversity of coral endosymbionts (zooxanthellae) from three Palauan reefs with contrasting bleaching, temperature and shading histories. Mol. Ecol. 13: 2445-2458

Fabricius KE, Alderslade P, Williams GC, Colin PL, Golbuu Y. (2007) Octocorallia in Palau, Micronesia: effects of biogeography and coastal influences on local and regional biodiversity. Pages 79 - 91 in Kayanne H, Omori M, Fabricius KE, Verheij E, Colin P, Golbuu Y, Yurihira H (eds) Coral Reefs of Palau. Palau International Coral Reef Centre, Palau

Fagoonee I, Wilson HB, Hassell MP, Turner JR. (1999) The dynamics of zooxanthellae populations: a long-term study in the field. Science. 283: 843-845. DOI: $10.1126 /$ science.283.5403.843

Falkowski PG, Dubinsky Z. (1981) Light-shade adaptation of Stylophora pistillata, a hermatypic coral from the Gulf of Eliat. Nature. 289: 172-174

Fay SA, Weber MX. (2012) The occurrence of mixed infections of Symbiodinium (Dinoflagellata) within individual hosts. J. Phycol. 48: 1306-1316

Feder JL. (1998) The apple maggot fly, Rhagoletis pomonella: flies in the face of conventional wisdom about speciation? In Endless Forms: Species and Speciation (Howard D, Berlocher S., eds), pp. 130144, Oxford University Press

Felsenstein J. (1985) Confidence limits on phylogenies: an approach using the bootstrap. Evolution. 39(4): 783-791

Fitt WK, Cook CB. (2001) The effects of feeding or addition of dissolved inorganic nutrients in maintaining the symbiosis between dinoflagellates and a tropical marine cnidarian. Mar. Biol. 139: $507-517$ 
Fitt WK, McFarland FK, Warner ME, Chilcoat GC. (2000) Seasonal patterns of tissue biomass and densities of symbiotic dinoflagellates in reef corals and relation to coral bleaching. Limnol. Oceanogr. 45(3): 677-685

Flatt T. (2005) The evolutionary genetics of canalization. Quart. Rev. Biol. 80(3): 287-316

Forsman ZH. (2003) Phylogeny and phylogeography of Porites and Siderastrea (Scleractinia: Cnidaria) species in the Caribbean and Eastern Pacific; based on the nuclear ribosomal ITS region. Ph.D. Dissertation. University of Houston, USA.

Forsman ZH, Concepcion GT, Haverkort RD, Shaw RW, Maragos JE, Toonen RJ. (2010) Ecomorph or endangered coral? DNA and microstructure reveal Hawaiian species complexes: Montipora dilatata/flabellatelturgescens \& M. patula/verrilli. PLoS ONE. 5(12): 1-10, doi:10.1371/journal.pone.0015021

France SC. (2007) Genetic analysis of bamboo corals (Cnidaria: Octocorallia: Isididae): does lack of colony branching distinguish Lepidisis from Keratoisis? Bull. Mar. Sci. 81(3): 323-333

France SC, Hoover LL. (2001) Analysis of variation in mitochondrial DNA sequences (ND3, ND4L, MSH) among Octocorallia (=Alcyonaria) (Cnidaria: Anthozoa). Bull. Biol. Soc. Wash. 10:110-118

Gaither MR, Rocha LA. (2013) Origins of species richness in the Indo-Malay-Philippine biodiversity hotspot: evidence for the centre of overlap hypothesis. J.Biogeogr. 40: 1638-1648

Garland T, Kelly SA. (2006) Phenotypic plasticity and experimental evolution. J. Exp. Biol. 209: 23442361

Garrabou J, Perez T, Sartoretto S, Harmelin JG. (2001) Mass mortality event in red coral Corallium rubrum populations in the Provence region (France, NW Mediterranean). Mar. Ecol. Prog. Ser. 217: 263-272

Gaston KJ, Blackburn TM, Spicer JI. (1998) Rapoport's rule: time for an epitaph? Trends Ecol. Evol. 13(2): 70-74

Gateño D, Barki Y, Rinkevich B. (1998) Aquarium maintenance of reef octocorals raised from field collected larvae. Aquar. Sci. Cons. 2: 227-236 
Gates RD, Hoegh-Guldberg O, McFall-Ngai MJ, Bil KY, Muscatine L. (1995) Free amino acids exhibit anthozoan "host factor" activity: They induce the release of photosynthate from symbiotic dinoflagellates in vitro. Proc. Natl. Acad. Sci. USA. 92: 7430-7434

Geiser DM, Taylor JW, Ritchie KB, Smith GW. (1998) Cause of sea-fan death in the West Indies. Nature. 394: 137-138

Gieskes WWC, Kraay GW, Nontji A, Setiapermana D, Sutomo D. (1988) Monsoonal alterations of a mixed and a layer structure in the phytoplankton of the euphotic zone of the Banda sea (Indonesia); a mathematical analysis of algal pigment fingerprints. Neth. J. Sea. Res. 22: 123-137

Gilbert SF, McDonald E, Boyle N, Buttino N, Gyi L, Mai M, Prakash N, Robinson J. (2010) Symbiosis as a source of selectable epigenetic variation: taking the heat for the big guy. Phil. Trans. R. Soc. B. 365: $671-678$

Gilbert SF, Epel D. (2009) Ecological developmental biology. Sinauer Associates, Sunderland, MA.

Goatley CHR, Bellwood DR. (2011) The roles of dimensionality, canopies and complexity in ecosystem monitoring. PLoS ONE. 6(11): e27307. doi:10.1371/journal.pone.0027307

Goh NKC, Chou LM. (1994) Distribution and biodiversity of Singapore gorgonians (sub-class Octocorallia) - a preliminary survey. Hydrobiolica. 285: 101-109

Goh NKC, Chou LM. (1996) An annotated checklist of the gorgonians (Anthozoa: Octocorallia) of Singapore, with a discussion of gorgonian diversity in the Indo-West Pacific. Raff. Bull. Zool. 44: 435-459

Goh NKC, Ng, PKL, Chou LM. (1999) Notes on the shallow water gorgonian-associated fauna on coral reefs in Singapore. Bull. Mar. Sci. 65(1): 259-282

Goldberg WM. (1973) The ecology of the coral-octocoral communities off the southeast Florida coast: geomorphology, species composition, and zonation. Bull. Mar. Sci. 23(3): 465-488

Gordon I. (1926) Notes on a number of muriceid genera (Alcyonaria, Gorgonaceae), with special reference to spiculation. Proceedings of the scientific meetings of the Zoological Society of London, pp 509-531

Gotthard K, Sören Nylin S. (1995) Adaptive Plasticity and Plasticity as an Adaptation: A Selective Review of Plasticity in Animal Morphology and Life History. Oikos. 74(1): 3-17 
Gouhier TC, Guichard F. (2007) Local disturbance cycles and the maintenance of heterogeneity across scales in marine metapopulations. Ecology. 88(3): 647-657

Gould SJ, Lewontin RC. (1979) The spandrels of San Marco and the Panglossian paradigm: a critique of the adaptationist programme. Proc. R. Soc. Lond. B. 205: 581-598

Gould SJ. (1997) The exaptive excellence of spandrels as a term and prototype. Proc. Natl. Acad. Sci. USA. 94: 10750-10755

Goulet TL. (2006) Most Corals may not change their symbionts. Mar. Ecol. Prog. Ser. 321: 1-7

Goulet TL, Coffroth MA. (2003) Stability of an octocoral-algal symbiosis over time and space. Mar. Ecol. Prog. Ser. 250: 117-124

Goulet TL, Simmons C, Goulet D. (2008) Worldwide biogeography of Symbiodinium in tropical octocorals. Mar. Ecol. Prog. Ser. 355: 45-58

Grajales A, Aguilar C, Sánchez JA. (2007) Phylogenetic reconstruction using secondary structures of Internal Transcribed Spacer 2 (ITS2, rDNA): finding the molecular and morphological gap in Caribbean gorgonian corals. BMC Evol. Biol. 7: 90

Grant R. (1976) The marine fauna of New Zealand: Isididae (Octocorallia: Gorgonacea) from New Zealand and the Antarctic. N. Z. Oceanogr. Inst. Mem. 66: 1-54

Grasshoff M. (1996) Zwei neue Gorgonarien-Arten von Neu Caledonien (Coelenterat", Octocorallia). Senckenbergiana bioi, 76 (1/2): 185-J90; Frankfurt am Main.

Grasshoff M. (1999) The shallow water gorgonians of New Caledonia and adjacent islands (Coelenterata: Octocorallia). Sencken. Biol. 78(1/2): 1-245

Grasshoff M. (2000) The gorgonians of the Sinai coast and the Strait of Gubal, Red Sea (Coelenterata, Octocorallia). Courier Forschung. Sencken. 224: 1-123

Grasshoff M, Zibrowius H. (1983) Kalkkrusten auf Achsen von Hornkorallen, rezent und fossil (Cnidaria, Anthozoa, Gorgonaria). Sencken. Mar. 15: 111-145

Grasshoff M, Bargibant G. (2001) Coral Reef Gorgonians of New Caledonia. IRD Editions Institut de Recherche pour le Développement. Paris. pp.336 
Gray JE. (1857) Synopsis of the families and genera of axiferous zoophytes or barked corals. Proc. Zool. Soc. Lond. 25: 278-94

Green DH, Edmunds PJ, Pochon X, Gates RD. (2010) The effects of substratum type on the growth, mortality, and photophysiology of juvenile corals in St. John, US Virgin Islands. J. Exp. Mar. Biol. Ecol. 384: 18-29

Grigg RW. (1972) Orientation and growth form of sea fans. Limnol. Oceanogr. 17(2): 185-192

Grigg RW. (1974) Growth rings: Annual periodicity in two gorgonian corals. Ecology. 55(4): 876-881

Grigg RW. (2001) Black Coral: History of a sustainable fishery in Hawai'i. Pac. Sci. 55(3): 291-299

Grigg RW. (2002) Precious corals in Hawai'i: discovery of a new bed and revised management measures for existing beds. Mar. Fish. Rev. 64(1): 13-20

Grillo MC, Goldberg WM, Allemand D. (1993) Skeleton and sclerite formation in the precious red coral Corallium rubrum. Mar. Biol. 117: 119-128

Guindon S, Gascuel O. (2003) A simple, fast, and accurate algorithm to estimate large phylogenies by maximum likelihood. System. Biol. 52: 696-704

Gutiérrez-Rodríguez C, Barbeitos MS, Sánchez JA, Lasker HR. (2009) Phylogeography and morphological variation of the branching octocoral Pseudopterogorgia elisabethae. Mol. Phyl. Evol. 50: $1-15$

Haapkylä J, Seymour AS, Trebilco J, Smith DJ. (2007) Coral disease prevalence and coral health in the Wakatobi Marine National Park, south-east Sulawesi, Indonesia. J. Mar. Biol. Ass. UK. 87: 403-414, doi:10.1017/S0025315407055828

Hageman SJ, Bayer MM, Todd CD. (1999) Partitioning phenotypic variation: genotypic, environmental and residual components from bryozoan skeletal morphology. J. Nat. Hist. 33(11): 1713-1735

Harrington L, Fabricius K, De'ath G, Negri A. (2004) Recognition and selection of settlement substrata determine post-settlement survival in corals. Ecology. 85(12): 3428-3427

Hay ME. (1986) Associational plant defenses and the maintenance of species diversity: turning competitors into accomplices. Am. Nat. 128: 617-641 
Helm C, Schülke I. (2003) An almost complete specimen of the Late Cretaceous (Campanian) octocoral 'Isis' ramosa Voigt (Gorgonacea) from the Lowe Saxony Basin, northwest Germany. Cret. Res. 24: $35-40$

Hennige SJ, Smith DJ, Perkins R, Consalvey M, Paterson DD, Suggett DJ. (2008a) Photoacclimation, growth and distribution of massive coral species in clear and turbid waters. Mar. Ecol. Prog. Ser. 369: 77-88

Hennige SJ, Suggett DJ, Warner ME, McDougall KE, Smith DJ. (2008b) Unravelling coral photoacclimation: Symbiodinium strategy and host modification. Proceedings of the 11th International Coral Reef Symposium. Ft. Lauderdale, Florida.

Hennige SJ, Suggett DJ, Warner ME, McDougall KE, Smith DJ. (2009) Photobiology of Symbiodinium revisited: bio-physical and bio-optical signatures. Coral Reefs. 28: 179-195

Hennige SJ, Smith DJ, Walsh SJ, McGinley MP, Warner ME, Suggett DJ. (2010) Acclimation and adaptation of scleractinian coral communities along environmental gradients within an Indonesian reef system. J. Exp. Mar. Biol. Ecol. 391: 143-152

Hereford J. (2009) A quantitative survey of local adaptation and fitness trade-offs. Am. Nat. 173(5): 579588

Herrera S, Baco A, Sanchez JA. (2010) Molecular systematics of the bubblegum coral genera (Paragorgiidae, Octocorallia) and description of a new deep-sea species. Mol. Phylogenet. Evol. 55(1): 123-135

Hodgson A. (2008) Spatial variation in reef echinoid abundance and diversity with habitat, fish community structure and anthropogenic pressure. Bachelors Dissertation. Newcastle University. UK.

Hoegh-Guldberg O, Jones RJ. (1999) Photoinhibition and photoprotection in symbiotic dinoflagellates from reef-building corals. Mar. Ecol. Prog. Ser. 183: 73-86

Hoeksema BW. (2007) Delineation of the Indo-Malayan Centre of Maximum Marine Biodiversity: The Coral Triangle. pp. 117-178. In: Biogeography, Time \& Place: Distributions, Barriers \& Islands. (Renema W, ed.) Springer, Netherlands.

Hoogenboom MO, Connolly SR, Anthony KRN. (2008) Interactions between morphological and physiological plasticity optimize energy acquisition in corals. Ecology. 89(4): 1144-1154 
Huelsenbeck JP, Ronquist F (2001) MRBAYES: Bayesian inference of phylogenetic trees. Bioinformatics. 17(8): 754-5

Hughes TP, Bellwood DR, Connolly SR. (2002) Biodiversity hotspots, centres of endemicity, and the conservation of the coral reefs. Ecol. Lett. 5: 775-784

Hurst LD (2009) Genetics and the understanding of selection. Nat. Rev. Genet.10: 83-93

Johnson C. (1976) Introduction to natural selection. University Park Press, Baltimore. pp. 213

Jokiel PL. (1978) Effects of water motion on reef corals. J. Exp. Mar. Biol. Ecol. 35: 87-97

Jones AM, Berkelmans R, van Oppen MJH, Sinclair W. (2008) A community change in the algal endosymbionts of a scleractinian coral following a natural bleaching event: field evidence of acclimatization. Proc. R. Soc. Lond. B. 275: 1359-1365

Jukes TH, Cantor CR. (1969) Evolution of protein molecules. (Munro H N, ed.) Mammalian protein metabolism, III. New York: Academic Press. pp. 21-132

Kaandorp JA, Kübler J. (2001) The Algorithmic Beauty of Seaweeds, Sponges and Corals. Springer, Amsterdam.

Kawecki TJ, Ebert D. (2004) Conceptual issues in local adaptation. Ecol. Let. 7: 1225-1241. doi: 10.1111/j.1461-0248.2004.00684.x

Kelmo F, Attrill MJ. (2001) Cnidarian community structure of coastal reefs from northern Bahia, Brazil. Bull. Mar. Sci. 69(2): 547-557

Kelmo F, Attrill MJ, Jones MB. (2003) Effects of the 1997-98 El Niňo on the cnidarian community of a high turbidity coral reef system (northern Bahia, Brazil). Coral Reefs. 22: 541-550, doi:10.1007/s00338-003-0343-0

Kim K, Lasker HR. (1997) Flow-mediated resource competition in the suspension feeding gorgonian Plexaura homomalla (Esper). J. Exp. Mar. Biol. Ecol. 215(1): 49-64

Kim E, Lasker HR, Coffroth MA, Kim K. (2004) Morphological and genetic variation across reef habitats in a broadcast-spawning octocorals. Hydrobiologica. 530/531: 423-432 
Kingsford M, Battershill C. (1998) Studying temperate marine environments: A hand book for ecologists. Canterbury University Press, New Zealand

Kinzie RA. (1973) The zonation of West Indian gorgonians. Bull. Mar. Sci. 23: 93-155

Kirk JTO. (1994) Light and photosynthesis in aquatic ecosystems. 2d ed. Cambridge University Press, Cambridge.

Knowlton N. (1993) Sibling species in the sea. Ann. Rev. Ecol. Sys. 24: 189-216

Kölliker RA. (1865) Die Bindesubstanz der Coelenteraten. Icones histologicae oder Atlas der vergleichenden Gewebelehre. Leipzig, Germany, pp. 87-181

Komada T, Anderson MR, Dorfmeier CL. (2008) Quantifying organic carbon and nitrogen concentration and isotopic compositions in carbonate-dominated coastal marine sediments. Limnol. Oceanogr. Methods. 6: 254-262

Koop K, Booth D, Broadbent A, Brodie J, Bucher D, Capone D, Coll J, Dennison W, Erdmann M, Harrison P, Hoegh-Guldberg O, Hutchings P, Jones GB, Larkum AWD, O’Neil J, Steven A, Tentori E, Ward S, Williamson J, Yellowlees. (2001) ENCORE: The effect of nutrient enrichment on coral reefs. Synthesis of results and conclusions. Mar. Poll. Bull. 42(2): 91-120

Kükenthal W. (1915) Das System der Seefedern. Zool. Anz. 45(6): 284-287

Kükenthal W. (1919) Gorgonaria. Wissenschaft. Ergebn. Deutsch. Tiefsee-Exspedition auf dem Dampfer Valdivia 1898-1899, Band 13, 946 pp

Kükenthal W. (1924) Coelenterata: Gorgonaria. Das Tierreich 47. Berlin: Walter de Gruyter \& Co. pp. 478

Kumagai NH. (2008) Role of food source and predator avoidance in habitat specialization by an octocoral-associated amphipod. Oecologia. 155: 739-749

Ladner JT, Palumbi SR. (2012) Extensive sympatry, cryptic diversity and introgression throughout the geographic distribution of two coral species complexes. Mol. Ecol. 21: 2224-2238

LaJeunesse TC. (2002) Diversity and community structure of symbiotic dinoflagellates from Caribbean coral reefs. Mar. Biol. 141: 387-400. (doi:10.1007/s00227-002-0829-2) 
LaJeunesse TC. (2005) 'Species' radiations of symbiotic dinoflagellates in the Atlantic and Indo-Pacific since the Miocene-Pliocene transition. Mol. Biol. Evol. 22: 570-581. (doi:10.1093/molbev/msi042)

LaJeunesse TC, Thornhill DJ. (2011) Improved resolution of reef-coral endosymbiont (Symbiodinium) species diversity, ecology, and evolution through $p s b A$ non-coding region genotyping. PLoS ONE. 6(12): e29013

LaJeunesse TC, Loh WKW, Woesik RV, Hoegh-Guldberg O, Schmidt GW, Fitt WK. (2003) Low symbiont diversity in southern Great Barrier Reef corals, relative to those of the Caribbean. Limnol. Oceanogr. 48: 2046-2054. (doi:10.4319/1o.2003.48.5.2046)

LaJeunesse TC, Bhagooli R, Hidaka M, DeVantier L, Done T, Schmidt GW, Fitt WKW, HoeghGuldberg O. (2004a) Closely related Symbiodinium spp. differ in relative dominance in coral reef host communities across environmental, latitudinal and biogeographic gradients. Mar. Ecol. Prog. Ser. 284: 147-161. (doi:10.3354/meps284147)

LaJeunesse TC, Thornhill D, Cox E, Stanton F, Fitt WKW, Schmidt G. (2004b) High diversity and host specificity observed among symbiotic dinoflagellates in reef coral communities from Hawaii. Coral Reefs. 23: 596-603. (doi:10.1007/s00338-004-0428-4)

LaJeunesse TC, Bonilla HR, Warner ME, Wills M, Schmidt GW, Fitt WK. (2008) Specificity and stability in high latitude eastern Pacific coral-algal symbioses. Limnol. Oceanogr. 53(2): 719-727

LaJeunesse TC, Smith RT, Finney J, Oxenford H. (2009) Outbreak and persistence of opportunistic symbiotic dinoflagellates during the 2005 Caribbean mass coral "bleaching" event. Proc. R. Soc. Lond. B. 276: 4139-4148

LaJeunesse TC, Parkinson JE, Reimer JD. (2012) A genetics-based description of Symbiodinium minutum sp. nov. and S. psygmophilum sp. nov. (Dinophyceae), two dinoflagellates symbiotic with Cnidaria. J. Phycol. 0: 1-12. DOI: 10.1111/j.1529-8817.2012.01217.x

Lamouroux JVF. (1812) Sur la classification des Polypiers corraligenes non entierement pierreux. Nouv. Bull. Soc. Philomath. Paris. 3: 181-8

Langer M. (1989) Haftorgan, internodien und sclerite von Keratoisis melitensis (Goldfuss 1826) (Octocorallia) in den pliozänen Foraminiferenmergeln (》Trubi《) von Milazzo (Sizilien). Paläont. Z. $63: 1 / 2: 15-24$ 
Larsen PE, Collart FR, Field D, Meyer F, Keegan KP, Henry CS, McGrath J, Quinn J, Gilbert JA. (2011a). Predicted Relative Metabolomic Turnover (PRMT): determining metabolic turnover from a coastal marine metagenomic dataset. BMC MIE. 1, 4. doi:10.1186/2042-5783-1-4

Larsen PE, Sreedasyam A, Trivedi G, Podila GK, Cseke LJ, Collart FR. (2011b) Using next generation transcriptome sequencing to predict an ectomycorrhizal metabolome. BMC Sys. Biol. 5: 70. doi:10.1186/1752-0509-5-70

Lasker HR, Boller ML, Castanaro J, Sánchez JA. (2003) Determinate growth and modularity in a gorgonian octocoral. Biol. Bull. 205: 319-330

Latypov YY. (2006) Transplantation and cultivation of fragments of coral colonies of various scleractinian species on a reef in Vietnam. Rus. Mar. Biol. 32(6): 375-381, doi: $10.1134 /$ S1063074006060071

Lesser MP, Farrell JH. (2004) Exposure to solar radiation increases damage to both host tissues and algal symbionts of corals during thermal stress. Coral Reefs. 23: 367-377

Lesser MP, Slattery M, Stat M, Ojimi M, Gates RD, Grottoli A. (2010) Photoacclimatization by the coral Montastraea cavernosa in the mesophotic zone: light, food, and genetics. Ecology. 91(4): 990-1003

Lesser MP, Stat M, Gates RD. (2013) The endosymbiotic dinoflagellates (Symbiodinium sp.) of corals are parasites and mutualists. Coral Reefs. DOI 10.1007/s00338-013-1051-z

Levene H. (1953) Genetic equilibrium when more than one ecological niche is available. Am. Nat. 87: $331-333$

Librado P, Rozas J. (2009) DnaSP v5: a software for comprehensive analysis of DNA polymorphism data. Bioinformatics. 25:1451-1452

Linares C, Coma R, Garrabou J, Díaz D, Zabala M. (2008) Size distribution, density and disturbance in two Mediterranean gorgonians: Paramuricea clavata and Eunicella singularis. J. Appl. Ecol. 45:688699

Lindstrom M. (1978) An octocoral from the Lower Ordovician of Sweden. Geol. Palaeont. 12(2): 41-52

MacIntyre IG, Rutzler K, Norris JN, Smith KP, Cairns SD, Bucher KE, Steneck RS. (1991) An early Holocene reef in the western Atlantic: submersible investigations of a deep relict reef off the west coast of Barbados, WI. Coral Reefs. 10: 167-174 
Magwene PM. (2001a) Comparing ontogenetic trajectories using growth process data. Syst. Biol. 50(5): $640-656$

Magwene PM. (2001b) New tools for studying integration and modularity. Evolution. 55(9): 1734-1745

Mai-Bao-Thu F, Domantay JS. (1970) Taxonomic studies of the Philippine gorgonaceans in the collections of the University of Santo Tomas, Manila. Acta Manilana. 6: 25-78

Mai-Bao-Thu F, Domantay JS. (1971) Taxonomic studies of the Philippine gorgonaceans in the collections of the University of Santo Tomas, Manila (cont'd). Acta Manilana. 7: 3-77

Matsumoto AK. (2004) Heterogeneous and compensatory growth in Melithaea flabellifera (Octocorallia: Melithaeidae) in Japan. Hydrobiologia. 530/531: 389-397

Matsumoto AK. (2007) Effects of low water temperature on growth and magnesium carbonate concentrations in deepwater gorgonians (Primnoa pacifica). Bull. Mar. Sci. 81: 423-435

Mattila TM, Bokma F. (2008) Extant mammal body masses suggest punctuated equilibrium. Proc. R. Soc. Lond. B. 275: 2195-2199

McArdle BH, Anderson MJ. (2001) Fitting multivariate models to community data: a comment on distance-based redundancy analysis. Ecology. 82: 290-297

McCloskey LR, Muscatine L. (1984) Production and respiration in the Red Sea coral Stylophora pistillata as a function of depth. Proc. R. Soc. Lond. B. 222: 215-230

McCormick MI. (1994) Comparison of field methods for measuring surface topography and their associations with tropical reef fish assemblage. Mar. Ecol. Prog. Ser. 112: 87-96

McCulloch M, Trotter J, Montagna P, Falter J, Dunbar R, Freiwald A, Försterra G, López Correa M, Maier C, Rüggeberg A, Taviani M. (2012) Resilience of cold-water scleractinian corals to ocean acidification: Boron isotopic systematics of $\mathrm{pH}$ and saturation state up-regulation. Geo. Cos. Acta. 87: $21-34$

McFadden CS. (1986) Colony fission increases particle capture rates of a soft coral: advantages of being a small colony. J. Exp. Mar. Biol. Ecol. 103: 1-20

McFadden CS, Donahue R, Hadland BK, Weston R. (2001) A molecular phylogenetic analysis of reproductive trait evolution in the soft coral genus Alcyonium. Evolution. 55: 54-67 
McFadden CS, Alderslade P, van Ofwegen LP, Johnsen H. Rusmevichientong A. (2006) Phylogenetic relationships within the tropical soft coral genera Sarcophyton and Lobophytum (Anthozoa, Octocorallia). Invert. Biol. 125(4): 288-305

McFadden CS, Sánchez JA, France SC. (2010a) Molecular Phylogenetic Insights into the Evolution of Octocorallia: A Review. Integ. Comp. Biol. 1-22

McFadden CS, Benayahu Y, Pante E, Thoma JN, Andrew N, France SC. (2010b) Limitations of mitochondrial gene barcoding in Octocorallia. Mol. Ecol. Res. 1755-0998

McLean EL, Yoshioka PM. (2007) Associations and interactions between gorgonians and sponges. Porifera Res. Biod. Inn. Sust. 443-448

Meesters EH, Hilterman M, Kardinaal E, Keetman M, de Vries M, Bak RPM. (2001) Colony sizefrequency distributions of scleractinian coral populations: spatial and interspecific variation. Mar. Ecol. Prog. Ser. 209: 43-54

Michod RE, Roze D. (1997) Transition in individuality. Proc. R. Soc. Lond. Ser. B. 264: 853-857

Middlebrook R, Hoegh-Guldberg O, Leggat W. (2008) The effect of thermal history on the susceptibility of reef-building corals to thermal stress. J. Exp. Biol. 211: 1050-1056 (doi:10.1242/jeb.013284)

Milne-Edwards H, Haime J. (1857) Histoire naturelle des coralliaires, ou polypes proprement dits. Paris, Roret. pp. 326

Molinier J, Ries G, Zipfel G, Hohn B. (2006) Transgeneration memory of stress in plants. Nature. 442: 1046-1049. (doi:10.1038/nature05022)

Mortensen PB, Buhl-Mortensen L. (2004) Distribution of deep-water gorgonian corals in relation to benthic habitat features in the Northeast Channel (Atlantic Canada). Mar. Biol. 144: 1223-1238

Muscatine L, Porter JW, Kaplan IR. (1989) Resource partitioning by reef corals as determined from stable isotope composition. I. $\delta^{13} \mathrm{C}$ of zooxanthellae and animal tissue vs depth. Mar. Biol. 100: 185193.

Muzik KM. (1979) Systematic revision of the Hawaiian Paramuriceidae and Plexauridae. Thesis, University of Miami, Coral Gables, Florida, USA, pp 227 
Muzik K, Wainwright SA. (1977) Morphology and habitat of five Fijian sea fans. Bull. Mar. 16(4): 277287

Naidoo K, Steenkamp ET, Coetzee MPA, Wingfield MJ, Wingfield BD. (2013) Concerted evolution in the ribosomal RNA citron. PLOS ONE. 8(3): e59355. doi:10.1371/journal.pone.0059355

Nei M. (2007) The new mutation theory of phenotypic evolution. Nat. Acad. Sci. USA. 104(30): 1223512242

Nelson FE, Wilson GS, Neil HL. (2013) Marine magnetic signature of the Last Glacial Maximum and last deglaciation from the Southern Hemisphere mid-latitudes. Mar. Geol. 346: 246-255

Noé SU, Dullo W-Chr. (2006) Skeletal morphogenesis and growth mode of modern and fossil deepwater isidid gorgonians (Octocorallia) in the West Pacific (New Zealand and Sea of Okhotsk). Coral Reefs. 25: 303-320. DOI 10.1007/s00338-006-0095-8

Nutting CC. (1910a). The Gorgonacea of the Siboga Expedition III. The Muriceidae. Siboga-Expeditie Monographie Monographie XIII, 108.pls. 1_22

Nutting CC. (1910b). The Gorgonacea of the Siboga Expedition IV. The Plexauridae. Siboga-Expeditie Monographie Monographie XIII, 20. pls. 1_4

Nutting CC. (1910c). The Gorgonacea of the Siboga Expedition V. The Isidae. Siboga- Expeditie Monographie XIII, 24. pls. 1_6

Nutting CC. (1910d). The Gorgonacea of the Siboga Expedition VI. The Gorgonellidae. SibogaExpeditie Monographie XIII, 39. pls. 1_11

Nutting CC. (1910e). The Gorgonacea of the Siboga Expedition VII. The Gorgoniidae. Siboga-Expeditie Monographie Monographie XIII, 10. pls. 1_3

Nutting CC. (1911). The Gorgonacea of the Siboga Expedition VIII. The Scleraxonia. Siboga-Expeditie Monographie Monographie XIII, 62. pls. 1_12

Oleson K. (2011) TEEB case: Taking an ecosystem service perspective in Velondriake locally managed marine area. Available at: TEEBweb.org

Oleson K, Oliver T, Raberinary D. (in prep.) Direct economic effects of temporary octopus closures in southwest Madagascar. 
Oliver T, Oleson K, Benbow S, Raberinary D. (in prep.) The biological closure effect of temporary octopus closures in southwest Madagascar.

Palumbi SR, Vollmer S, Romano S, Oliver T, Ladner J. (2011) The role of genes in understanding the evolutionary ecology of reef building corals. Evol. Ecol. 26: 317-335

Pante E, France SC, Couloux A, Cruaud C, McFadden CS, Samadi S, Watling L. (2012) Deep-sea origin and in-situ diversification of Chrysogorgiid Octocorals. PLoS ONE. 7(6): e38357. doi:10.1371/journal.pone.0038357

Paulay G, Puglisi MP, Starmer JA. (2003) The non-scleractinian Anthozoa (Cnidaria) of the Mariana Islands. Micronesica. 35-36: 138-155

Peet RK. (1974) The measurement of species diversity. Ann. Rev. Ecol. Sys. 5: 285-307

Pemberton AJ, Noble LR, Bishop JDD. (2003) Frequency dependence in matings with water-borne sperm. J. Evol. Biol. 16: 304-316

Pfenning DW, Wund MA, Snell-Rood EC, Cruickshank T, Schlichting CD, Moczek AP. (2010) Phenotypic plasticity's impacts on diversification and speciation. TREE. 25: 459-467

Picciano M, Ferrier-Pagès C. (2007) Ingestion of pico- and nanoplankton by the Mediterranean red coral Corallium rubrum. Mar. Biol. 150: 773-782

Pigliucci M. (2005) Evolution of phenotypic plasticity: where are we going now? Trends Ecol. Evol. 20: $481-486$

Pigliucci M, Murren CJ, Schlichting CD. (2006) Phenotypic plasticity and evolution by genetic assimilation. J. Exp. Biol. 209: 2362-2367

Pilgrim SE, Cullen LC, Smith DJ, Pretty J. (2007a) Hidden Harvest or Hidden Revenue? Local resource use in a remote region of Southeast Sulawesi, Indonesia. Ind. J. Trad. Knowl. 6(1): 150-159

Pilgrim SE, Smith D, Pretty J. (2007b) A cross-regional quantitative assessment of the factors affecting ecoliteracy: Policy and practice implications. Ecol. App. 17(6): 1742-1751

Pochon X, Gates RD. (2010) A new Symbiodinium clade (Dinophyceae) from soritid foraminifera in Hawai'i. Mol. Phylogenet. Evol. 56: 492-497. (doi:10.1016/j.ympev.2010.03.040) 
Pochon X, Pawlowski J, Zaninetti, Rowan R. (2001) High genetic diversity and relative specificity among Symbiodinium-like endosymbiotic dinoflagellates in soritid foraminiferans. Mar. Biol. 139: 1069-1078

Pochon X, Putnam HM, Burki F, Gates RD. (2012) Identifying and Characterizing Alternative Molecular Markers for the Symbiotic and Free-Living Dinoflagellate Genus Symbiodinium. PLoS ONE. 7(1): e29816. doi:10.1371/journal.pone.0029816

Pont-Kingdon G, Okada NA, Macfarlane JL, Beagley CT, Wolstenholme DR, Cavalier-Smith T, ClarkWalker GD. (1995) A coral mitochondrial MutS gene. Nature. 375: 109-111

Pont-Kingdon G, Okada NA, Macfarlane JL, Beagley CT, Watkins-Sims CD, Cavalier-Smith T, ClarkWalker GD, Wolstenholme DR. (1998) Mitochondrial DNA of the coral Sarcophyton glaucum contains a gene for a homologue of bacterial MutS: a possible case of gene transfer from the nucleus to the mitochondrion. J. Mol. Evol. 46: 419-413

Poorter H, Nagel OW. (2000) The role of biomass allocation in the growth response of plants to different levels of light, $\mathrm{CO}_{2}$, nutrients and water: a quantitative review. Aus. J. Plant Phys. 27: 595-607

Porra RJ, Thompson WA, Kriedemann PE. (1989) Determination of accurate extinction coefficients and simultaneous equations for assaying chlorophylls $a$ and $b$ extracted with four different solvents: verification of the concentration of chlorophyll standards by atomic absorption spectroscopy. Bioch. Biophys. Acta. 975: 384-394

Porter JW. (1976) Autotrophy, heterotrophy, and resource partitioning in Caribbean reef-building corals. Am. Nat. 110(975): 731-742

Porter JW, Muscatine L, Dubinsky Z, Falkowski PG. (1984) Primary production and photoadaptation in light- and shade-adapted colonies of the symbiotic coral Stylophora pistillata. Proc. R. Soc. Lond. B. 222: $161-180$

Powell AL, Hepburn LJ, Smith DJ, Bell JJ. (2010) Patterns of sponge abundance across a gradient of habitat quality in the Wakatobi Marine National Park, Indonesia. Open Mar. Biol. J. 4: 31-38

Prada C, Schizas NV, Yoshioka PM. (2008) Phenotypic plasticity or speciation? A case of a clonal marine organism. BMC EVOL. Biol. 8(47): 1-19

Prada C, Hellberg ME. (2013) Long prereproductive selection and divergence by depth in a Caribbean candelabrum coral. Proc. Nat. Acad. Sci. USA. 110: 3961-3966 
Puglisi MP, Paul VJ, Slattery M. (2000) Biogeographic comparisons of chemical and structural defenses of the Pacific gorgonians Annella mollis and A. reticulate. Mar. Ecol. Prog. Ser. 207: 263-272

Puritz JB, Toonen RJ. (2011) Coastal pollution limits pelagic larval dispersal. Nat. Commun. 2: 226 (doi:10.1038/ncomms1238)

Puritz JB, Addison JA, Toonen RJ. (2012a) Next-generation phylogeography: a targeted approach for multilocus sequencing of non-model organisms. PLoS ONE. 7(3): e34241. doi:10.1371/journal.pone.0034241

Puritz JB, Keever CC, Addison JA, Byrne M, Hart MW, Grosberg RK, Toonen RJ. (2012b) Extraordinarily rapid life-history divergence between Cryptasterina sea star species. Proc. R. Soc. Lond. B. (doi:10.1098/rspb.2012.1343)

Putnam HM, Stat M, Pochon X, Gates RD. (2012) Endosymbiotic flexibility associates with environmental sensitivity in scleractinian corals. Proc. R. Soc. Lond. B. 279:4352-4361

Pyle RL. (1996) A learner's guide to closed circuit rebreather diving. In: Menduno, M. (Ed.) Proceedings of the Rebreather Forum 2.0. 26-28 September, 1996. Redondo Beach, CA. DSAT, Santa Ana, CA. pp P45-P67

Pyle RL, Earle JL, Green BD. (2008) Five new species of the damselfish genus Chromis (Perciformes: Labroidei: Pomacentridae) from deep coral reefs in the tropical western Pacific. Zootaxa. 1671: 3-31

Reitzal AM, Sullivan JC, Traylor-Knowles N, Finnerty JR. (2008) Genomic survey of candidate stressresponse genes in the estuarine anemone Nematostella vectensis. Biol. Bull. 214: 233-254

Rice SH. (2008) Theoretical approaches to the evolution of development and genetic architecture. Ann. N.Y. Acad. Sci. 1133: 67-86

Riedl R, Forstner H. (1968) Wasserbewegung im Mikrobereich des Benthos. Sarsia. 34: 163-188

Riegl B, Branch GM. (1995) Effects of sediment on the energy budgets of four scleractinian (Bourne 1900) and five alcyonacean (Lamouroux 1816) corals. J. Exp. Mar. Biol. Ecol. 186: 259-275

Risk M, Heikoop J, Snow M, Beukens R. (2002) Life spans and growth patterns of two deep-sea corals: Primnoa resedaeformis and Desmophyllum cristagalli. Hydrobiologia. 471: 125-131 
Risk MJ, Lapointe BE, Sherwood OA, Bedford BJ. (2009) The use of $\delta^{15} \mathrm{~N}$ in assessing sewage stress on coral reefs. Mar. Poll. Bull. 58: 793-802. doi:10.1016/j.marpolbul.2009.02.008

Ritchie RJ. (2006) Consistent sets of spectrophotometric chlorophyll equations for acetone, methanol and ethanol solvents. Photosynth. Res. 89: 27-41

Roark E, Guilderson T, Dunbar R, Ingram B. (2006) Radiocarbon based ages and growth rates: Hawaiian Deep Sea Corals. Mar. Ecol. Prog. Ser. 327: 1-14

Rodríguez SR, Ojeda FP, Inestrosa NC. (1993) Settlement of benthic marine invertebrates. Mar. Ecol. Prog. Ser. 97: 193-207

Rodriguez-Lanetty M, Phillips WS, Weis VM. (2006) Transcriptome analysis of a cnidariandinoflagellate mutualism reveals complex modulation of host gene expression. BMC Genomics. 7: 23

Rodriguez-Lanetty M, Phillips WS, Dove S, Hoegh-Guldberg O, Weis VM. (2008) Analytical approach for selecting normalizing genes from a cDNA microarray platform to be used in q-RT-PCR assays: A cnidarian case study. J. Biochem. Biophys. Methods. 70: 985-991

Rogers AD, Gianni M. (2010) The implementation of UNGA Resolutions 61/105 and 64/72 in the management of deep-sea fisheries on the high seas. Report Prepared for the Deep-Sea Conservation Coalition, International Programme on the State of the Ocean, 97.

Rohde K. (1992) Latitudinal gradients in species diversity: the search for the primary cause. Oikos. 65 : $514-527$

Rohde K. (1999) Latitudinal gradients in species diversity and Rapoport's rule revisited: a review of recent work and what can parasites teach us about the causes of the gradients? Ecography. 22: 593613

Rogers CS. (1990) Responses of coral reefs and reef organisms to sedimentation. Mar. Ecol. Prog. Ser. 62: $185-202$

Rosen BR. (1988) Progress, problems and patterns in the biogeography of reef corals and other tropical marine organisms. Helgol. Meer. 42: 269-301

Roth LV. (1996) Cranial Integration in the Sciuridae. Amer. Zool. 36: 14-23

Rowan R. (2004) Thermal adaptation in reef coral symbionts. Nature. 430: 742 
Rowan R, Knowlton N. (1995) Intraspecific diversity and ecological zonation in coral-algal symbiosis. Proc. Natl. Acad. Sci. 92: 2850-2853

Rowan R, Knowlton N, Baker AC, Jara J. (1997) Landscape ecology of algal symbionts creates variation in episodes of coral bleaching. Nature. 388: 265-269

Rowley SJ, Watling L, Pochon X, Gates RD, Smith DJ, Bell JJ. (2011) Gorgonian responses to environmental change on coral reefs in SE Sulawesi, Indonesia. $22^{\text {nd }}$ Pacific Science Conference.

Rundle H, Nosil P. (2005) Ecological speciation. Ecol. Lett. 8: 336-352

Sachs JL, Essenberg CJ, Turcotte MM. (2011) New paradigms for the evolution of beneficial infections. Trends Ecol. Evol. 26: 202-209

Salter-Cid L, Bigger CH. (1991) Alloimmunity in the Gorgonian Coral Swiftia exserta. Biol. Bull. 181: $127-134$

Sánchez JA. (2004) Evolution and dynamics of branching colonial form in marine modular cnidarians: gorgonian octocorals. Hydrobiologia. 00: 1-8

Sánchez JA. (2007) A new genus of Atlantic octocorals (Octocorallia: Gorgoniidae): systematics of gorgoniids with asymmetric sclerites. J. Nat. Hist. 41: 493-509

Sánchez JA, Diaz JM, Zea S. (1997) Gorgonian communities in two contrasting environments on oceanic atolls of the southwest Caribbean. Bull. Mar. Sci. 61(2): 453-465

Sánchez JA, Diaz JM, Zea S. (1998) Octocoral and black coral distribution patterns on the barrier reefcomplex of Providencia island, Southwestern Caribbean. Caribb. J. Sci. 34(3-4): 250-264

Sánchez JA, Lasker HR. (2003) Patterns of morphological integration in marine modular organisms: supra-module organization in branching octocoral colonies. Proc. R. Soc. Lond. B. 270: 2039-2044

Sánchez JA, McFadden CS, France SC. (2003a) Phylogenetic analyses among octocorals (Cnidaria): mitochondrial and nuclear DNA sequences (1su-rRNA, 16S and ssu-rRNA, 18S) support two convergent clades of branching gorgonians. Mol. Phylogen. Evol. 29: 31-42

Sánchez JA, McFadden CS, France SC, Lasker HR. (2003b) Molecular phylogenetic analyses of shallow-water Caribbean octocorals. Mar. Biol. 142: 975-987 
Sánchez JA, Zeng W, Coluci VR, Simpson C, Lasker R. (2003c) How similar are branching networks in nature? A view from the ocean: Caribbean gorgonian corals. J. Theor. Biol. 222: 135-138

Sánchez JA, Lasker HR. (2004) Do multi-branched colonial organisms exceed normal growth after partial mortality? Proc. R. Soc. B. (Suppl.). 271: S117-120

Sánchez JA, Aguilar C, Dorado D, Manrique N. (2007) Phenotypic plasticity and morphological integration in a marine modular invertebrate. BMC Evol. Biol. 7(112): 1-9

Sanciangco JC, Carpenter KE, Etnoyer PJ, Moretzsohn F. (2013) Habitat availability and heterogeneity and the Indo-Pacific warm pool as predictors of marine species richness in the tropical Indo-Pacific. PLoS ONE. 8(2): e56245. doi:10.1371/journal.pone.0056245

Santelices B. (1999) How many kinds of individuals are there? Trends Ecol. Evol. 14(4): 152-155

Scaps P, Denis V. (2007) Association between the scallop, Pedum spondyloideum, (Bivalva: Pteriomorphia: Pectinidae) and scleractinian corals from the Wakatobi Marine National Park (southeastern Sulawesi, Indonesia). Raff. Bull. Zool. 55: 371-380

Schlichter D, Fricke HW, Weber W. (1986) Light harvesting by wavelength transformation in asymbiotic coral of the Red Sea twilight zone. Mar. Biol. 91: 403-407

Schlichting CD. (1986) The evolution of phenotypic plasticity in plants. Ann. Rev. Ecol. Syst. 17: 667-93

Schlichting CD, Pigliucci M. (1998) Phenotypic Evolution: A Reaction Norm Perspective. Sunderland, MA: Sinauer Associates

Schluter D. (1998) Ecological causes of speciation. In Endless Forms: Species and Speciation (Howard D. and Berlocher S., eds), pp. 114-129, Oxford University Press

Schluter D. (2001) Ecology and the origin of species. Trends Ecol. Evol. 16(7): 372-380

Schoener TW. (1974) Resource partitioning in ecological communities. Science. 185: 27-39

Schoenberg DA, Trench RK. (1980) Genetic variation in Symbiodinium (Gymnodinium) microadriaticum Freudenthal, and specificity in its symbiosis with marine invertebrates. III. Specificity and infectivity of Symbiodinium microadriaticum. Proc. R. Soc. Lond. B. 207: 445- 460 
Sebens KP. (1982) The limits to indeterminate growth: an optimal size model applied to passive suspension feeders. Ecol. 63: 209-222

Sebens KP. (1984) Water flow and coral colony size: Inter habitat comparisons of the octocoral Alcyonoum siderium. Proc. Natl. Acad. Sci. 81: 5473-5477

Sebens KP. (1997) Adaptive responses to water flow: morphology, energetics, and distribution of reef corals. Proc. $8^{\text {th }}$ Int. Coral Reef Sym. 2:1053-1058

Sebens KP, Witting J, Helmuth B. (1997) Effects of water flow and branch spacing on particle capture by the coral reef coral Madracis mirabilis (Duchassaing and Michelotti). J. Exp. Mar. Biol. Ecol. 211 : $1-28$

Seibel PN, Muller T, Dandekar T, Schultz J, Wolf M. (2006) 4SALE - a tool for synchronous RNA sequence and secondary structure alignment and editing. BMC Bioinform. 7: 498

Seibel P, Muller T, Dandekar T, Wolf M. (2008) Synchronous visual analysis and editing of RNA sequence and secondary structure alignments using 4SALE. BMC Res. Notes. 1: 91

Shaish L, Abelson A, Rinkevich B. (2006) Branch to colony trajectory in a modular organism: pattern formation in the Indo-Pacific coral Stylophora pistillata. Dev. Dyn. 235: 2111-2121

Shea K, Roxburgh H, Rauschert ESJ. (2004) Moving from pattern to process: coexistence mechanisms under intermediate disturbance regimes. Ecol. Lett. 7: 491-508. doi: 10.1111/j.14610248.2004.00600.x

Sheppard CR. (1979) Interspecific aggression between reef corals with reference to their distribution. Mar. Ecol. Prog. Ser. 1: 237-247

Silverstein RN, Correa AMS, Baker AC. (2012) Specificity is rarely absolute in coral-algal symbiosis: implications for coral response to climate change. Proc. R. Soc. B. 279: 2609-2618

Simmons MP, Ochoterena H. (2000) Gaps as characters in sequence-based phylogenetic analyses. Syst. Biol. 49(2): 369-381

Simpson JJ. (1906) The structure of Isis hippuris, Linnaeus. J Linn. Soc. Lon. Zool. 29(194): 421-434

Simpson C. (2013) Species selection and the macroevolution of coral coloniality and photosymbiosis. Evolution. 67-6: 1607-1621 
Skoufas G. (2006) Comparative biometry of Eunicella singurlaris (Gorgonian) sclerites at East Mediterranean Sea (North Aegean Sea, Greece). Mar. Biol. 149: 1365-1370

Smith GW, Ives LD, Nagelkerken IA, Ritchie KB. (1996) Caribbean sea fan mortalities. Nature. 383: 487

Sorokin YI. (1991) Biomass, metabolic rates and feeding of some common reef zooantharians and octocorals. Aust. J. Mar. Freshwater Res. 42: 729-41

Sponaugle S, LaBarbera M. (1991) Drag-induced deformation: a functional feeding strategy in two species of gorgonians. J. Exp. Mar. Biol. Ecol. 148: 121-134

Stachowitsch M. (1992) The invertebrates: an illustrated glossary. Wiley-Liss, Inc., New York. pp. 676

Stafford-Smith MG. (1993) Sediment-rejection efficiency of 22 species of Australian scleractinian corals. Mar. Biol. 115: 229-243

Stambler N, Dubinsky Z. (2005) Corals as light collectors: an integrating sphere approach. Coral Reefs. 24: $1-9$

Stat M, Gates RD. (2011) Clade D Symbiodinium in scleractinian corals: a "nugget" of hope, a selfish opportunist, an ominous sign, or all of the above? J. Mar. Biol. doi:10.1155/2011/730715

Stat M, Bird CE, Pochon X, Chasqui L, Chauka LJ, Concepcion GT, Logan D, Takabayahsi M, Toonen RJ, Gates RD. (2011) Variation in Symbiodinium ITS2 sequence assemblages among coral colonies. PLOS ONE. 6: e15854.

Stears SC, de Jong G, Newman R. (1991) The effects of pheno-typic plasticity on genetic correlations. Trends Ecol. Evol. 6: 122-126

Stiasny G. (1937). Die Gorgonacea der Siboga-Expedition. Suppl. II, Revision der Scleraxonia, etc. Siboga-Expeditie. Monographie $13 b^{8}$ [Livr. 130]. vi +133 pp. 8 pls.

Stiasny G. (1940). Biological results of the Snellius Expedition. VII. Die Gorgonarien Sammlung der Snellius-Expedition, Temminckia 5: 191-256, 15 figs, 9 pls.

Stolarski J, Kitahara MV, Miller DJ, Cairns SD, Mazur M, Meibom A. (2011) The ancient evolutionary origins of Scleractinia revealed by azooxanthellate corals. BMC Evol. Biol. 11:316. doi:10.1186/1471-2148-11-316 
Suggett DJ, Le Floc'H E, Harris GN, Leonardos N, Geider RJ. (2007) Different strategies of photoacclimation by two strains of Emiliania huxleyi (Haptophyta). J. Phycol. 43: 1209-1222

Susilaningsih N, Murwani R, Trianto A, Widagdo, Purnianto A, Anisatusholihah. (2009) The effects of gorgonian Isis hippuris extract on proliferative activity and histological grading of adenocarcinoma mammae cells in $\mathrm{C} 3 \mathrm{H}$ mice. In: International Seminar and Workshop on Modern Biology and its applications: Focusing on Stem Cells and Human Genetics, Semarang, Indonesia.

Takabayashi M, Santos SR, Cook CB. (2004) Mitochondrial DNA phylogeny of the symbiotic dinoflagellates (Symbiodinium, Dinophyta). J. Phycol. 40: 16-164

Taylor EB, Boughman JW, Groenenboom M, Sniatynski M, Schluter D, Gow JL. (2006) Speciation in reverse: morphological and genetic evidence of the collapse of a three-spined stickleback (Gasterosteus aculeatus) species pair. Mol. Ecol. 15: 343-355

Thomson JA, Simpson JJ. (1909) An account of the alcyonarians collected by the Royal Indian Marine Survey Ship Investigator in the Indian Ocean; with a report on the species of Dendronephthya by Henderson WD II. The alcyonarians of the littoral area. The Indian Museum, Calcutta

Thompson JD, Gibson TJ, Higgins DG. (2002) Multiple sequence alignment using ClustalW and ClustalX. Curr Protoc Bioinformatics Chapter 2: Unit 23

Thresher RE, Wilson NC, MacRae CM, Neil H. (2010) Temperature effects on the calcite skeletal composition of deep-water gorgonians (Isididae). Geochim. Cosmochim. Ac. 74: 4655-4670

Tinsley P. (2005) Worbarrow reefs seafan project. Dorset Wildlife Trust report.

Toller WW, Rowan R, Knowlton N. (2001a) Zooxanthellae of the Montastraea annularis species complex: patterns of distribution of four taxa of Symbiodinium on different reefs and across depths. Biol. Bull. 201: 348-359

Toller WW, Rowan R, Knowlton N. (2001b) Repopulation of zooxanthellae in the Caribbean corals Montastraea annularis and M. faveolata following experimental and disease-associated bleaching. Biol. Bull. 201: 360-373

Tomasick T, Mah AJ, Nontji A, Kasim Moosa M. (2004) The ecology of the Indonesian seas part one. Oxford University Press. pp. 642 
Townsend CR, Scarsbrook MR. (1997) The intermediate disturbance hypothesis, refugia, and biodiversity in streams. Limnol. Oceanogr. 42(5): 938-949

Turelli M, Barton NH, Coyne JA. (2001) Theory and speciation. Trends Ecol. Evol. 16: 330-343

Unsworth RK, Powell A, Hukom F, Smith DJ. (2007) The ecology of Indo-Pacific grouper (Serranidae) species and the effects of a small scale no take area on grouper assemblage, abundance and size frequency distribution. Mar. Biol. 74: 53-62

Valenciennes A. (1846) Zoophytes. In: Abel Dupetit-Thouars. Voyage autour du monde sur la fre' gate la Ve' nus, pendant les anne's 1836-1839. Atlas de Zoologie, pp. 1-15

Valenciennes A. (1855) Extrait d'une monographie de la familie des Gorgonide' es de la classe des polypes. C.R. Acad. Sci. Paris 41: 7-15

van Ofwegen L. (2004) The present status of taxonomic knowledge of octocorals in Indonesia. In The ecology of the Indonesian seas part one. Tomasick T, Mah AJ, Nontji A, Kasim Moosa M. Oxford University Press. pp. 345-346

van Oppen MJH, Mieog CJ, Sánchez CA Fabricius KE. (2005) Diversity of algal endosymbionts (zooxanthellae) in octocorals: the roles of geography and host relationships. Mol. Ecol. 14: 24032417

van Soest RWM. (1979) A catalogue of the Coelenterate type specimens of the Zoological Museum of Amsterdam. IV. Gorgonacea, Actinaria, Scleractinia. Beaufortia. 29(353): 81-126

Veron JEN. (1995) Corals in space and time: the biography and evolution of the scleractinian. Ithaca, NY: Cornell University Press.

Veron JEN, Devantier LM, Turak E, Green AL. (2009) Delineating the Coral Triangle. Galaxea.19: 91100

Verseveldt J. (1966). Biological Results of the Snellius Expedition XXII. Octocorallia from the Malay Archipelago (Part II). Zoo logische Verhandelingen Leiden. 80: 1-109

Versluys J. (1902). Die Gorgoniden der Siboga Expedition I. Die Chrysogorgiidae. Siboga-Expeditie Monographie XIII, 120 
Versluys J. (1906). Die Gorgoniden der Siboga Expedition II. Die Primnoidae. Siboga- Expeditie Monographie XIII, 178. pls. 1_10

Vogel S. (1983) Life in moving fluids: the physical biology of flow. Princeton University Press, Princeton, New Jersey. 352p.

Vollmer SV, Palumbi SR. (2002) Hybridization and the evolution of reef coral diversity. Science. 296: 2023-2025

Waggoner B, Collins AG. (2004) Reductio ad absurdum: testing the evolutionary relationships of Ediacaran and Paleozoic problematic fossils using molecular divergence dates. J. Paleont. 78(1): 5161

Wald A, Wolfowitz J. (1943) An exact test for randomness in the non-parametric case based on serial correlation. Ann. Math. Stat. 14: 378-388

Warner ME, Chilcoat GC, McFarland FK, Fitt WK. (2002) Seasonal fluctuations in the photosynthetic capacity of photosystem II in symbiotic dinoflagellates in the Caribbean reef-building coral Montastraea. Mar. Biol. 141: 31-38. DOI 10.1007/s00227-002-0807-8

Watling L, Norse EA. (1998) Disturbance of the seabed by mobile fishing gear: a comparison to forest clear cutting. Conserv. Biol. 12: 1180-1197

Watling L, France SC, Pante E, Simpson A. (2011) Biology of deep-water octocorals. Adv. Mar. Biol. 60: 41-122

Watling L, Guinotte J, Clark MR, Smith CR. (2012) A proposed biogeography of the deep ocean floor. Prog. Ocean. 111: 91-112

Watling L, France SC, Rowley SJ, Heestand E. (2012) When is a bamboo coral not a bamboo coral? A revision of the octocoral family Isididae. Proc. $5^{\text {th }}$ Int. Deep Sea Coral Symposium

Webber H. (2008) An Examination of Reactive or Adaptive Livelihood Strategies in a Time of Increasing Vulnerability due to Long Term Declining Natural Resources: A case study of the Bajo living on Sampela in the Wakatobi Marine National Park, Sulawesi, Indonesia. Masters Dissertation: Kings College London, UK.

Weersing K, Toonen RJ. (2009) Population genetics, larval dispersal, and connectivity in marine systems. Mar. Ecol. Prog. Ser. 393: 1-12 
Weil E. (2004) Coral reef diseases in the wider Caribbean. In Coral health and disease. Rosenberg E, Loya Y (Eds). Springer, New York. pp. 35-64

Weinbauer MG, Velimirov BV. (1995) Morphological variations in the Mediterranean sea fan Eunicella cavolini (Coelenterata: Gorgonacea) in relation to exposition, colony size and colony region. Bull. Mar. Sci. 56: 283-295

Weinbauer MG, Velimirov BV. (1996) Population Dynamics and overgrowth of the sea fan Eunicella cavolini (Coelenterata: Octocorallia). Est. Coast. Shelf. Sci. 42: 583-595

Weinbauer MG, Velimirov BV. (1998) Comparative morphometry of fan-like colonies of three Mediterranean gorgonians (Cnidaria: Gorgonacea). Cahi. Biol. Mari. 39: 41-49

Weiner J. (2004) Allocation, plasticity and allometry in plants. Per. Plant Ecol. Evol. Syst. 6: 207-215

Weir JT, Schluter D. (2007) The latitudinal gradient in recent speciation and extinction rates of birds and mammals. Science. 315: 1574-1576

Wentworth CK. (1922) "A Scale of Grade and Class Terms for Clastic Sediments.” J. Geology. 30: 377392

West JM. (1997) Plasticity in the sclerites of a gorgonian coral: tests of water motion, light level, and damage cues. Biol. Bull. 192: 279-289

West JM. (1998) The dual role of sclerites in a gorgonian coral: conflicting functions of support and defence. Evol. Ecol. 12: 803-821

West JM, Harvell CD, Walls AM. (1993) Morphological plasticity in a gorgonian coral (Briareum asbestinum) over a depth cline. Mar. Ecol. Prog. Ser. 94: 61-69

West-Eberhard MJ. (1989) Phenotypic plasticity and the origins of diversity. Ann. Rev. Ecol. Sys. 20: 249-78

West-Eberhard MJ. (2003) Developmental Plasticity and Evolution. Oxford Univ Press, New York.

West-Eberhard MJ. (2005) Phenotypic accommodation: adaptive innovation due to developmental plasticity. J. Exp. Zool Mol. Dev. Evol. 304B: 610-618 
Weyl PK. (1964) On the change in electrical conductance of sea water with temperature. Limnol. Oceanog. 9: 75-78

Wilkerson FP, Kobayashi D, Muscatine L. (1988) Mitotic index and size of symbiotic algae in Caribbean Reef corals. Coral Reefs. 7: 29-36.

Wille A, Buhlmann P. (2006) Low-order conditional independence graphs for inferring genetic networks. Stat. App. Gen. Mol. Biol. 5: Article 1

Williams B, Grottoli AG. (2010) Stable nitrogen and carbon isotope $\left(\delta^{15} \mathrm{~N}\right.$ and $\left.\delta^{13} \mathrm{C}\right)$ variability in shallow tropical Pacific soft coral and black coral taxa and implications for paleoceanographic reconstructions. Geo. Cos. Acta. 74: 5280-5288

Williams GC, Cairns, SD. (2013) Biodiversity myth busters. In: Octocoral Research Center Website. <http://researcharchive.calacademy.org/research/izg/Biodiversity\%20Myth\%20Busters.html >.

Cited 9 December 2013

Williams GC, Delbeek JC, Shepherd B, Wolters S. (2010) Zooxanthellae in ellisellid gorgonians of the Philippines. Proc. Cal. Acad. Sci. 61(18): 647-648

Willis BL, Page CA, Dinsdale EA. (2004) Coral disease on the Great Barrier Reef. In Coral health and disease. Rosenberg E, Loya Y (Eds). Springer, New York. pp. 69-102

Wirshing HH, Messing CG, Douady CJ, Reed J, Stanhope MJ, Shivji MS. (2005) Molecular evidence for multiple lineages in the gorgonian family Plexauridae (Anthozoa: Octocorallia). Mar. Biol. 147: 497508

Wright EP, Studer TH. (1889) Report on the Alcyonaria. Rep. Scient. Results Explor. Voyage Challenger. 31(1): 1-314

$\mathrm{Xu}$ CY, Schooler SS, Van Klinken RD. (2012) Differential influence of clonal integration on morphological and growth responses to light in two invasive herbs. PLos ONE. 7(4): e35873

Yellowless D, Rees TA, Leggat W. (2008) Metabolic interactions between algal symbionts and invertebrate hosts. Plant. Cell. Environ. 31: 679-694. doi: 10.1111/j.1365-3040.2008.01802.x

Young ND, Healy J. (2003) GapCoder automates the use of indel characters in phylogenetic analysis. BMC Bioinf. 4:6 
Yoshioka PM, Yoshioka BB. (1989) Effects of water motion, topographic relief and sediment transport on the distribution of shallow-water gorgonian community. Mar. Ecol. Prog. Ser. 54: 257-264

Yoshioka PM. (1996) Variable recruitment and its effect on the population and community structure of shallow-water gorgonians. Bull. Mar. Sci. 59: 433-443

Zou R, Huang B, Wang X. (1991) Studies on the gorgonians of China - I. Isis with one new species. Acta. Oceano. Sinica. 10(4): 593-602

Zuker M. (2003) Mfold web server for nucleic acid folding and hybridization prediction. Nucl. Acids Res. 31: 3406-3415 